Supporting Information for

\title{
Insights into the Mechanistic Role of Diphenylphosphine Selenide, Diphenylphosphine, and Primary Amines in the Formation of CdSe Monomers
}

\author{
Ting Qi ${ }^{\mathrm{a}}$, Hua-Qing Yang ${ }^{\mathrm{a}^{*}}$, Dennis M. Whitfield ${ }^{\mathrm{c}}$, Kui Yu ${ }^{\mathrm{c}^{*}}$, Chang-Wei Hu ${ }^{\mathrm{b}}$ \\ Received (in $X X X, X X X)$ Xth $X X X X X X X X X 20 X X$, Accepted Xth $X X X X X X X X X 20 X X$ \\ DOI: 10.1039/b000000x
}

${ }^{a}$ College of Chemical Engineering, Sichuan University, Chengdu, Sichuan, 610065, P.R. China

${ }^{b}$ Key Laboratory of Green Chemistry and Technology, Ministry of Education, College of Chemistry, Sichuan

University, Chengdu, Sichuan, 610064, P.R. China

${ }^{c}$ College of Physics, Sichuan University, Chengdu, Sichuan, 610064, P.R. China

J. Phys. Chem. A

*Correspondence to: H.-Q. Yang; E-mail: huaqingyang@scu.edu.cn

K. Yu; E-mail: kuiyu@ @scu.edu.cn 


\section{Catalog}

i. $\quad$ Figure S1a. The geometric structures of various species, and the relative Gibbs free energies $\left(G_{\mathrm{r}}\right)\left(\mathrm{kJ} \mathrm{mol}^{-1}\right)$ relative to the discrete reactants calculated at the M06//B3LYP/6-31++G(d, p), SDD level are shown. Bond lengths are reported in A.

ii. Figure S1b. The geometric structures of various species, and the schematic energy diagrams for the reaction $\mathrm{SePR}_{3}+$ $\mathrm{HPPh}_{2} \rightarrow \mathrm{SePPh}_{2} \mathrm{H}+\mathrm{PR}_{3}$. Bond lengths are reported in $\AA$. Relative Gibbs free energies $\left(G_{\mathrm{r}}\right)\left(\mathrm{kJ} \mathrm{mol}^{-1}\right)$ for the corresponding species relative to $\mathrm{SePR}_{3}+\mathrm{HPPh}_{2}$ at the M06//B3LYP/6-31++G(d, p), SDD level are shown.

.P6

iii. Figure S2. The geometric structures of various species (a), and the schematic energy diagrams (b) for the reaction (3) $\mathrm{Cd}(\mathrm{OA})_{2}+\mathrm{SePPh}_{2} \mathrm{H} \rightarrow \mathbf{C}+\mathrm{RCOOH},(4) \mathrm{Cd}(\mathrm{OA})_{2}+\mathrm{HPPh}_{2} \rightarrow \mathbf{C}^{\prime}+\mathrm{RCOOH}$, and (5) $\mathrm{Cd}(\mathrm{OA})_{2}+\mathrm{RNH}_{2} \rightarrow \mathbf{C}^{\prime \prime}+$ $\mathrm{RCOOH}$. Bond lengths are reported in $\AA$. Relative Gibbs free energies $\left(G_{\mathrm{r}}\right)\left(\mathrm{kJ} \mathrm{mol}^{-1}\right)$ for the corresponding species relative to $\mathrm{Cd}(\mathrm{OA})_{2}+\mathrm{L}\left(\mathrm{L}=\mathrm{SePPh}_{2} \mathrm{H}, \mathrm{HPPh}_{2}\right.$, and $\left.\mathrm{RNH}_{2}\right)$ at the M06//B3LYP/6-31++G(d, p), SDD level are shown. Blue, red, and Green lines represent the reactions (3), (4), and (5), respectively.

iv. Figure S3. The geometric structures of various species (a), and the schematic energy diagrams (b) for the reaction $\mathrm{Cd}(\mathrm{OA})_{2}+\mathrm{SePPh}_{2} \mathrm{H}+\mathrm{HPPh}_{2} \rightarrow \mathbf{G}+2 \mathrm{RCOOH}$ calculated at the M06//B3LYP/6-31++G(d, p), SDD level are shown. Bond lengths are reported in $\AA$. Relative Gibbs free energies $\left(G_{\mathrm{r}}\right)\left(\mathrm{kJ} \mathrm{mol}^{-1}\right)$ for the corresponding species plus $\mathrm{RCOOH}$ form $\mathbf{C}+\mathrm{HPPh}_{2}$ to $\mathbf{G}$, for the corresponding species plus $\mathrm{HPPh}_{2}+\mathrm{RCOOH}-\mathrm{SePPh}_{2} \mathrm{H}$ from $\mathbf{F}+\mathrm{HPPH}_{2}$ to $\mathbf{E}-\mathbf{i}$, and for the corresponding species plus $2 \mathrm{RCOOH}-\mathrm{SePPh}_{2} \mathrm{H}$ from $\mathbf{E}$ and $\mathbf{E}-\mathbf{i}$ to $\mathbf{G}$ are shown relative to $\mathrm{Cd}(\mathrm{OA})_{2}+\mathrm{SePPh}_{2} \mathrm{H}+$ $\mathrm{HPPH}_{2}$. Green, red, and blue lines represent the reaction (8) $\mathbf{C}+\mathrm{HPPh}_{2} \rightarrow \mathbf{G}+\mathrm{RCOOH}$, (9) $\mathbf{E}+\mathrm{HPPh}_{2} \rightarrow \mathbf{G}+$ $\mathrm{SePPh}_{2} \mathrm{H}$, and (10) $\mathbf{F}+2 \mathrm{HPPh}_{2} \rightarrow \mathbf{G}+\mathrm{RCOOH}+\mathrm{SePPh}_{2} \mathrm{H}$, respectively.

v. Figure S4. The geometric structures of various species (a), and the schematic energy diagrams (b) for the reaction $\mathrm{Cd}(\mathrm{OA})_{2}+\mathrm{SePPh}_{2} \mathrm{H}+\mathrm{RNH}_{2} \rightarrow \mathbf{H}+2 \mathrm{RCOOH}$ calculated at the M06//B3LYP/6-31++G(d, p), SDD level are shown.. Bond lengths are reported in $\AA$. Relative Gibbs free energies $\left(G_{\mathrm{r}}\right)\left(\mathrm{kJ} \mathrm{mol}^{-1}\right)$ for the corresponding species plus $\mathrm{RCOOH}$ form $\mathbf{C}+\mathrm{RNH}_{2}$ to $\mathbf{H}$, for the corresponding species plus $\mathrm{HPPh}_{2}+\mathrm{RCOOH}-\mathrm{SePPh}_{2} \mathrm{H}$ from $\mathbf{F}+\mathrm{RNH}_{2}$ to $\mathbf{I}-\mathbf{i}$, for the corresponding species plus $2 \mathrm{RCOOH}-\mathrm{SePPh}_{2} \mathrm{H}$ from $\mathbf{I - i}$ and $\mathbf{E}$ to $\mathbf{H}$, and for the corresponding species plus $2 \mathrm{RCOOH}-$ $\mathrm{HPPh}_{2}$ from $\mathbf{G}+\mathrm{RNH}_{2}$ to $\mathbf{H}$ are shown relative to $\mathrm{Cd}(\mathrm{OA})_{2}+\mathrm{SePPh}_{2} \mathrm{H}+\mathrm{RNH}_{2}$. Green, red, blue, and black lines represent the reaction (13) $\mathbf{C}+\mathrm{RNH}_{2} \rightarrow \mathbf{H}+\mathrm{RCOOH}$, (14) $\mathbf{E}+\mathrm{RNH}_{2} \rightarrow \mathbf{H}+\mathrm{SePPh}_{2} \mathrm{H}$, (15) $\mathbf{F}+\mathrm{RNH}_{2}+\mathrm{HPPh}_{2} \rightarrow \mathbf{H}+$ $\mathrm{RCOOH}+\mathrm{SePPh}_{2} \mathrm{H}$, and (16) $\mathbf{G}+\mathrm{RNH}_{2} \rightarrow \mathbf{H}+\mathrm{HPPh}_{2}$, respectively.

vi. Figure S5. The geometric structures of various species (a), and the schematic energy diagrams (b) for the reaction $\mathrm{Cd}(\mathrm{OA})_{2}+2 \mathrm{SePPh}_{2} \mathrm{H}+\mathrm{RNH}_{2} \rightarrow \mathbf{I}+\mathrm{HPPh}_{2}+2 \mathrm{RCOOH}$ calculated at the M06//B3LYP/6-31++G(d, p), SDD level are shown. Bond lengths are reported in $\AA$. Relative Gibbs free energies $\left(G_{\mathrm{r}}\right)\left(\mathrm{kJ} \mathrm{mol}^{-1}\right)$ for the corresponding species plus $2 \mathrm{RCOOH}$ from $\mathbf{H}+\mathrm{SePPh}_{2} \mathrm{H}$ and $\mathbf{E}-\mathbf{i}+\mathrm{RNH}_{2}$ to $\mathbf{I}$, and for the corresponding species plus $\mathrm{HPPh}_{2}+2 \mathrm{RCOOH}$ from I-i to I are shown relative to $\mathrm{Cd}(\mathrm{OA})_{2}+2 \mathrm{SePPh}_{2} \mathrm{H}+\mathrm{RNH}_{2}$. Blue, red, and Green lines represent the reaction (21) $\mathbf{H}+$ $\mathrm{SePPh}_{2} \mathrm{H} \rightarrow \mathbf{I}+\mathrm{HPPh}_{2}, \quad(22) \quad \mathbf{I}-\mathbf{i} \rightarrow \mathbf{I}$, and $(23)$ E-i $+\mathrm{RNH}_{2} \rightarrow \quad \mathbf{I}+\mathrm{HPPh}_{2}$, respectively. P13

vii. Figure S6. The geometric structures and the schematic energy diagrams for the reaction $\mathrm{Cd}(\mathrm{OA})_{2}+\mathrm{SePPh}{ }_{2} \mathrm{H} \rightarrow \mathbf{C}+$ $\mathrm{RCOOH}$ calculated at the M06/6-31++G(d, p), SDD level in the ODE media under room temperature and atmospheric pressure $(298.15 \mathrm{~K}$ and $1 \mathrm{~atm})$. Relative Gibbs free energies $\left(G_{\mathrm{r}}\right)\left(\mathrm{kJ} \mathrm{mol}^{-1}\right)$ for the corresponding species relative to $\mathrm{Cd}(\mathrm{OA})_{2}+\mathrm{SePPh}_{2} \mathrm{H}$ are shown. Bond lengths are reported in $\AA$. Black and blue lines represent the level of M06/6-31++G(d, p), SDD and M06//B3LYP/6-31++G(d, p), SDD, respectively. .P15

viii. Table S1. The zero-point energies ( $Z P E$, hartree), thermal correction to Gibbs free energy $\left(G_{0}\right.$, hartree) of various species calculated at the B3LYP/6-31++G(d, p), SDD level in the gas phase under atmospheric pressure and room temperature (298.15 $\mathrm{K}$ and $1 \mathrm{~atm})$. Polarizable continuum model correction energies (PCM-E, hartree), total energies ( $E_{\mathrm{c}}$, hartree) corrected by $Z P E$, sum of electronic and thermal free energies $\left(G_{\mathrm{c}}\right.$, hartree) corrected by $G_{0}$, relative energies $\left(E_{\mathrm{r}}, \mathrm{kJ} \mathrm{mol}^{-1}\right)$ and relative Gibbs free energies $\left(G_{\mathrm{r}}, \mathrm{kJ} \mathrm{mol}^{-1}\right)$ to $\mathrm{Cd}(\mathrm{OA})_{2}+n \mathrm{~L}\left(\mathrm{~L}=\mathrm{SePR}_{3}, \mathrm{RNH}_{2}, \mathrm{SePPh}_{2} \mathrm{H}, \mathrm{PR}_{3}\right.$, and $\left.\mathrm{HPPh}_{2} ; n=1 \sim 2\right)$ for all the species in the reaction of $\mathrm{Cd}(\mathrm{OA})_{2}+n \mathrm{~L} \rightarrow \mathrm{L}_{n} \mathrm{Cd}(\mathrm{OA})_{2}$ calculated at the M06//B3LYP/6-31++G(d, p), SDD level in 1-octadecene solution 
under room temperature and atmospheric pressure (298.15 $\mathrm{K}$ and $1 \mathrm{~atm})$.

ix. Table S2. The zero-point energies (ZPE, hartree), thermal correction to Gibbs free energy $\left(G_{0}\right.$, hartree) of various species calculated at the B3LYP/6-31++G(d, p), SDD level in the gas phase under atmospheric pressure and room temperature (298.15 $\mathrm{K}$ and $1 \mathrm{~atm})$. Polarizable continuum model correction energies (PCM-E, hartree), total energies $\left(E_{\mathrm{c}}\right.$, hartree) corrected by $Z P E$, sum of electronic and thermal free energies $\left(G_{\mathrm{c}}\right.$, hartree) corrected by $G_{0}$, relative energies $\left(E_{\mathrm{r}}, \mathrm{kJ}^{\mathrm{mol}}{ }^{-1}\right)$ and relative Gibbs free energies $\left(G_{\mathrm{r}}, \mathrm{kJ} \mathrm{mol}^{-1}\right)$ to $\mathrm{Cd}(\mathrm{OA})_{2}+\mathrm{L}\left(\mathrm{L}=\mathrm{SePPh}_{2} \mathrm{H}, \mathrm{HPPh}_{2}, \mathrm{RNH}_{2}\right)$ for all the species in the reaction of $\mathrm{Cd}(\mathrm{OA})_{2}+\mathrm{SePPh}_{2} \mathrm{H}$ $\rightarrow \mathbf{C}+\mathrm{RCOOH}, \mathrm{Cd}(\mathrm{OA})_{2}+\mathrm{HPPh}_{2} \rightarrow \mathbf{C}^{\prime}+\mathrm{RCOOH}$, and $\mathrm{Cd}(\mathrm{OA})_{2}+\mathrm{RNH}_{2} \rightarrow \mathbf{C}^{\prime \prime}+\mathrm{RCOOH}$ calculated at the M06//B3LYP/6-31++G(d, p), SDD level in 1-octadecene solution under room temperature and atmospheric pressure (298.15 K and $1 \mathrm{~atm}$ ) .P17

x. Table S3. The zero-point energies ( $Z P E$, hartree), thermal correction to Gibbs free energy ( $G_{0}$, hartree) of various species calculated at the B3LYP/6-31++G(d, p), SDD level in the gas phase under atmospheric pressure and room temperature (298.15 $\mathrm{K}$ and $1 \mathrm{~atm})$. Polarizable continuum model correction energies (PCM-E, hartree), total energies ( $E_{\mathrm{c}}$, hartree) corrected by $Z P E$, sum of electronic and thermal free energies $\left(G_{\mathrm{c}}\right.$, hartree) corrected by $G_{0}$, relative energies $\left(E_{\mathrm{r}}, \mathrm{kJ}^{\mathrm{mol}}{ }^{-1}\right)$ and relative Gibbs free energies $\left(G_{\mathrm{r}}, \mathrm{kJ} \mathrm{mol}^{-1}\right)$ to $\mathrm{Cd}(\mathrm{OA})_{2}+2 \mathrm{SePPh}_{2} \mathrm{H}$ for all the species in the reaction of $\mathbf{C}+\mathrm{SePPh}_{2} \mathrm{H} \rightarrow \mathbf{E}+\mathrm{RCOOH}$ and $\mathbf{C}+$ $\mathrm{SePPh}_{2} \mathrm{H} \rightarrow \mathbf{F}+\mathrm{HPPh}_{2}$ calculated at the M06//B3LYP/6-31++G(d, p), SDD level in 1-octadecene solution under room temperature and atmospheric pressure $(298.15 \mathrm{~K}$ and $1 \mathrm{~atm})$.

xi. Table S4. The zero-point energies (ZPE, hartree), thermal correction to Gibbs free energy $\left(G_{0}\right.$, hartree) of various species calculated at the B3LYP/6-31++G(d, p), SDD level in the gas phase under atmospheric pressure and room temperature (298.15 $\mathrm{K}$ and $1 \mathrm{~atm})$. Polarizable continuum model correction energies (PCM-E, hartree), total energies $\left(E_{\mathrm{c}}\right.$, hartree) corrected by $Z P E$, sum of electronic and thermal free energies $\left(G_{\mathrm{c}}\right.$, hartree) corrected by $G_{0}$, relative energies $\left(E_{\mathrm{r}}, \mathrm{kJ} \mathrm{mol}^{-1}\right)$ and relative Gibbs free energies $\left(G_{\mathrm{r}}, \mathrm{kJ} \mathrm{mol}^{-1}\right)$ to $\mathrm{Cd}(\mathrm{OA})_{2}+\mathrm{SePPh}_{2} \mathrm{H}+\mathrm{HPPh}_{2}$ for all the species in the reaction of $\mathrm{Cd}(\mathrm{OA})_{2}+\mathrm{SePPh}_{2} \mathrm{H}+\mathrm{HPPh}_{2} \rightarrow$ $\mathbf{G}+2 \mathrm{RCOOH}\left(\mathbf{C}+\mathrm{HPPh}_{2} \rightarrow \mathbf{G}+\mathrm{RCOOH}, \mathbf{E}+\mathrm{HPPh}_{2} \rightarrow \mathbf{G}+\mathrm{SePPh}_{2} \mathrm{H}\right.$, and $\left.\mathbf{F}+2 \mathrm{HPPh}_{2} \rightarrow \mathbf{G}+\mathrm{RCOOH}+\mathrm{SePPh}{ }_{2} \mathrm{H}\right)$ calculated at the M06//B3LYP/6-31++G(d, p), SDD level in 1-octadecene solution under room temperature and atmospheric pressure $(298.15 \mathrm{~K}$ and $1 \mathrm{~atm})$.

xii. Table S5. The zero-point energies ( $Z P E$, hartree), thermal correction to Gibbs free energy $\left(G_{0}\right.$, hartree) of various species calculated at the B3LYP/6-31++G(d, p), SDD level in the gas phase under atmospheric pressure and room temperature (298.15 $\mathrm{K}$ and $1 \mathrm{~atm})$. Polarizable continuum model correction energies (PCM-E, hartree), total energies $\left(E_{\mathrm{c}}\right.$, hartree) corrected by $Z P E$, sum of electronic and thermal free energies $\left(G_{\mathrm{c}}\right.$, hartree) corrected by $G_{0}$, relative energies $\left(E_{\mathrm{r}}, \mathrm{kJ}^{\mathrm{mol}}{ }^{-1}\right)$ and relative Gibbs free energies $\left(G_{\mathrm{r}}, \mathrm{kJ} \mathrm{mol}^{-1}\right)$ to $\mathrm{Cd}(\mathrm{OA})_{2}+\mathrm{SePPh}_{2} \mathrm{H}+\mathrm{RNH}_{2}$ for all the species in the reaction of $\mathrm{Cd}(\mathrm{OA})_{2}+\mathrm{SePPh}_{2} \mathrm{H}+\mathrm{RNH}_{2} \rightarrow \mathbf{H}$ $+2 \mathrm{RCOOH}\left((13) \mathbf{C}+\mathrm{RNH}_{2} \rightarrow \mathbf{H}+\mathrm{RCOOH}\right.$, (14) $\mathbf{E}+\mathrm{RNH}_{2} \rightarrow \mathbf{H}+\mathrm{SePPh}_{2} \mathrm{H}$, (15) $\mathbf{F}+\mathrm{RNH}_{2}+\mathrm{HPPh}_{2} \rightarrow \mathbf{H}+\mathrm{RCOOH}+$ $\mathrm{SePPh}_{2} \mathrm{H}$, and (16) $\mathbf{G}+\mathrm{RNH}_{2} \rightarrow \mathbf{H}+\mathrm{HPPh}_{2}$ ) calculated at the M06//B3LYP/6-31++G(d, p), SDD level in 1-octadecene solution under room temperature and atmospheric pressure (298.15 K and $1 \mathrm{~atm})$.

xiii. Table S6. The zero-point energies ( $Z P E$, hartree), thermal correction to Gibbs free energy $\left(G_{0}\right.$, hartree) of various species calculated at the B3LYP/6-31++G(d, p), SDD level in the gas phase under atmospheric pressure and room temperature (298.15 $\mathrm{K}$ and $1 \mathrm{~atm})$. Polarizable continuum model correction energies (PCM-E, hartree), total energies $\left(E_{\mathrm{c}}\right.$, hartree) corrected by $Z P E$, sum of electronic and thermal free energies $\left(G_{\mathrm{c}}\right.$, hartree) corrected by $G_{0}$, relative energies $\left(E_{\mathrm{r}}, \mathrm{kJ}^{\mathrm{mol}}{ }^{-1}\right)$ and relative Gibbs free energies $\left(G_{\mathrm{r}}, \mathrm{kJ} \mathrm{mol}^{-1}\right)$ to $\mathrm{Cd}(\mathrm{OA})_{2}+2 \mathrm{SePPh}_{2} \mathrm{H}+\mathrm{RNH}_{2}$ for all the species in the reaction of $\mathrm{Cd}(\mathrm{OA})_{2}+2 \mathrm{SePPh}_{2} \mathrm{H}+\mathrm{RNH}_{2} \rightarrow$ $\mathbf{I}+\mathrm{HPPh}_{2}+2 \mathrm{RCOOH}\left(\mathbf{H}+\mathrm{SePPh}_{2} \mathrm{H} \rightarrow \mathbf{I}+\mathrm{HPPh}_{2}, \mathbf{I}-\mathbf{i} \rightarrow \mathbf{I}\right.$, and $\left.\mathbf{E}-\mathbf{i}+\mathrm{RNH}_{2} \rightarrow \mathbf{I}+\mathrm{HPPh}_{2}\right)$ calculated at the M06//B3LYP/6-31++G(d, p), SDD level in 1-octadecene solution under room temperature and atmospheric pressure (298.15 K and $1 \mathrm{~atm})$.... .P23

xiv. Table S7. Relative Gibbs free energies $\left(G_{\mathrm{r}}, \mathrm{kJ} \mathrm{mol}^{-1}\right)$ for the coordination of $\mathrm{RNH}_{2}, \mathrm{SePPh}_{2} \mathrm{H}, \mathrm{HPPh}$, and $\mathrm{RCOOH}$ ligands toward key intermediates (RCOOCdSe-PPh $(\mathbf{C}), \mathrm{RCOOCdSe}_{2} \mathrm{PPh}_{2}(\mathbf{F}), \mathrm{Ph}_{2} \mathrm{PSe}_{2} \mathrm{Cd}-\mathrm{SePPh}_{2}(\mathbf{E}), \mathrm{Ph}_{2} \mathrm{P}_{-} \mathrm{CdSe}_{2} \mathrm{PPh}_{2}(\mathbf{E}-\mathbf{i})$, $\mathrm{Ph}_{2} \mathrm{P}-\mathrm{CdSe}-\mathrm{PPh}_{2}(\mathbf{G}), \mathrm{RHN}-\mathrm{CdSe}_{2} \mathrm{PPh}_{2}(\mathbf{I}-\mathbf{i}), \mathrm{RHN}-\mathrm{CdSe}-\mathrm{PPh}_{2}(\mathbf{H})$, and $\left.\mathrm{Ph}_{2} \mathrm{PSe}-\mathrm{CdSe}-\mathrm{NHR}(\mathbf{I})\right)$, respectively, relative to the discrete reactants calculated at the M06//B3LYP/6-31++G(d, p), SDD level in 1-octadecene solution under room temperature and atmospheric pressure (298.15 $\mathrm{K}$ and $1 \mathrm{~atm})$. ..P24

xv. Figure S7. The geometric structures for the coordination of $\mathrm{RNH}_{2}, \mathrm{SePPh}_{2} \mathrm{H}, \mathrm{HPPh}_{2}$, and $\mathrm{RCOOH}$ toward important 
intermediates (RCOOCdSe- $\mathrm{PPh}_{2} \quad(\mathbf{C}), \quad \mathrm{RCOOCdSe} \mathrm{PPh}_{2} \quad(\mathbf{F}), \mathrm{Ph}_{2} \mathrm{PSe}_{2} \mathrm{Cd}-\mathrm{SePPh}_{2} \quad(\mathbf{E}), \quad \mathrm{Ph}_{2} \mathrm{P}-\mathrm{CdSe} \mathrm{PPh}_{2} \quad(\mathbf{E}-\mathbf{i})$, $\mathrm{Ph}_{2} \mathrm{P}-\mathrm{CdSe}-\mathrm{PPh}_{2}$ (G), RHN-CdSe${ }_{2} \mathrm{PPh}_{2}(\mathbf{I}-\mathbf{i}), \mathrm{RHN}-\mathrm{CdSe}-\mathrm{PPh}_{2}(\mathbf{H})$, and $\mathrm{Ph}_{2} \mathrm{PSe}-\mathrm{CdSe}-\mathrm{NHR}$ (I)) calculated at the M06//B3LYP/6-31++G(d, p), SDD level in 1-octadecene solution under room temperature and atmospheric pressure (298.15 K and $1 \mathrm{~atm})$.

xvi. Table S8. Snapshot and standard orientation of all the species in the gas phase calculated at the B3LYP/6-31++G(d, p), SDD level. Distance lengths are reported in $\AA$.

xvii. Figure S8. Arrhenius plots of calculated rate constants for the crucial reaction step. .P82

xviii. Table S9. Snapshot and standard orientation of all the species in the ODE solution calculated at the M06/6-31++G(d, p), SDD level. 


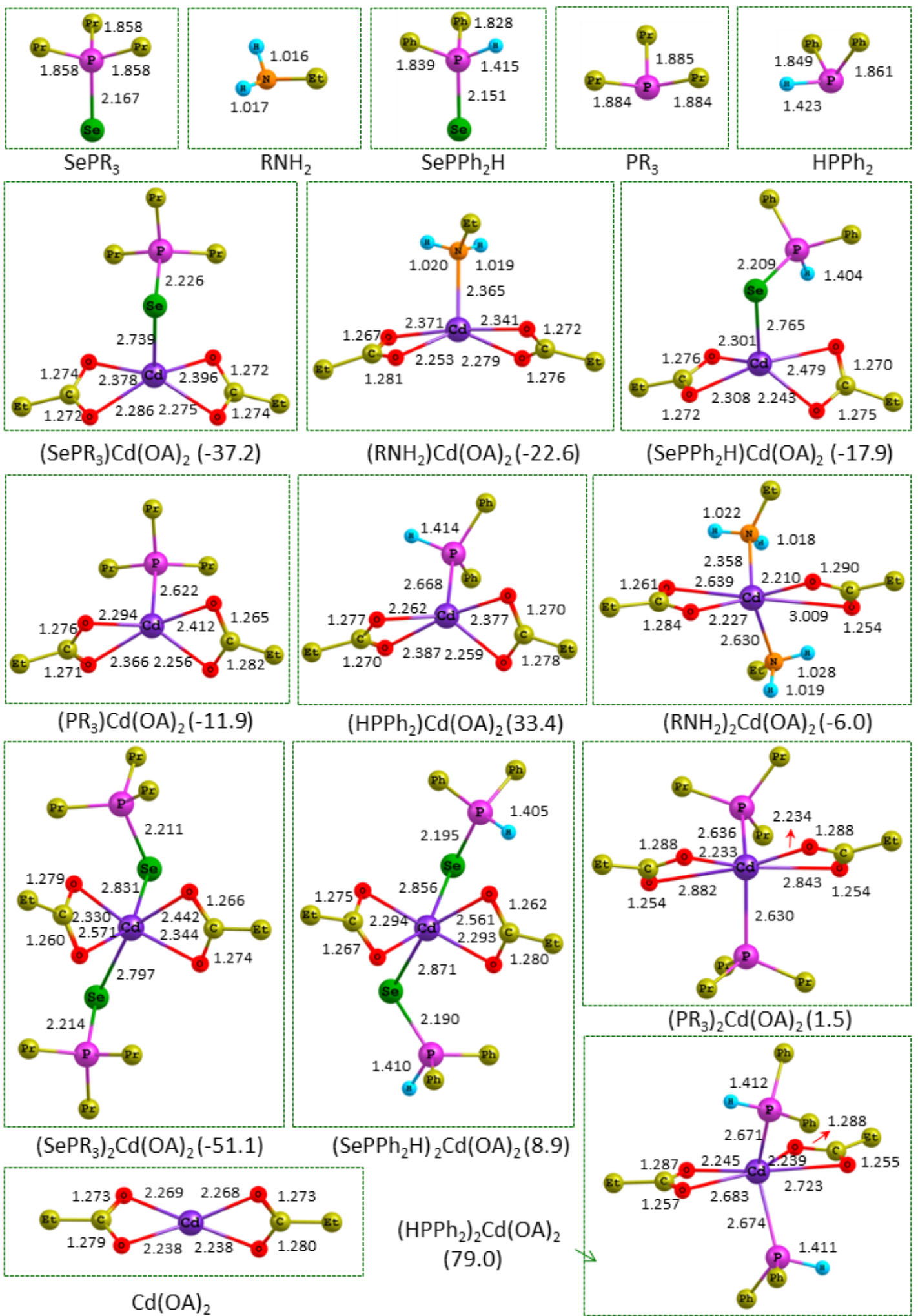

Figure S1a. The geometric structures of various species, and the relative Gibbs free energies $\left(G_{\mathrm{r}}\right)\left(\mathrm{kJ} \mathrm{mol}^{-1}\right)$ relative to the discrete reactants calculated at the M06//B3LYP/6-31++G(d, p), SDD level are shown. Bond lengths are reported in Å. 


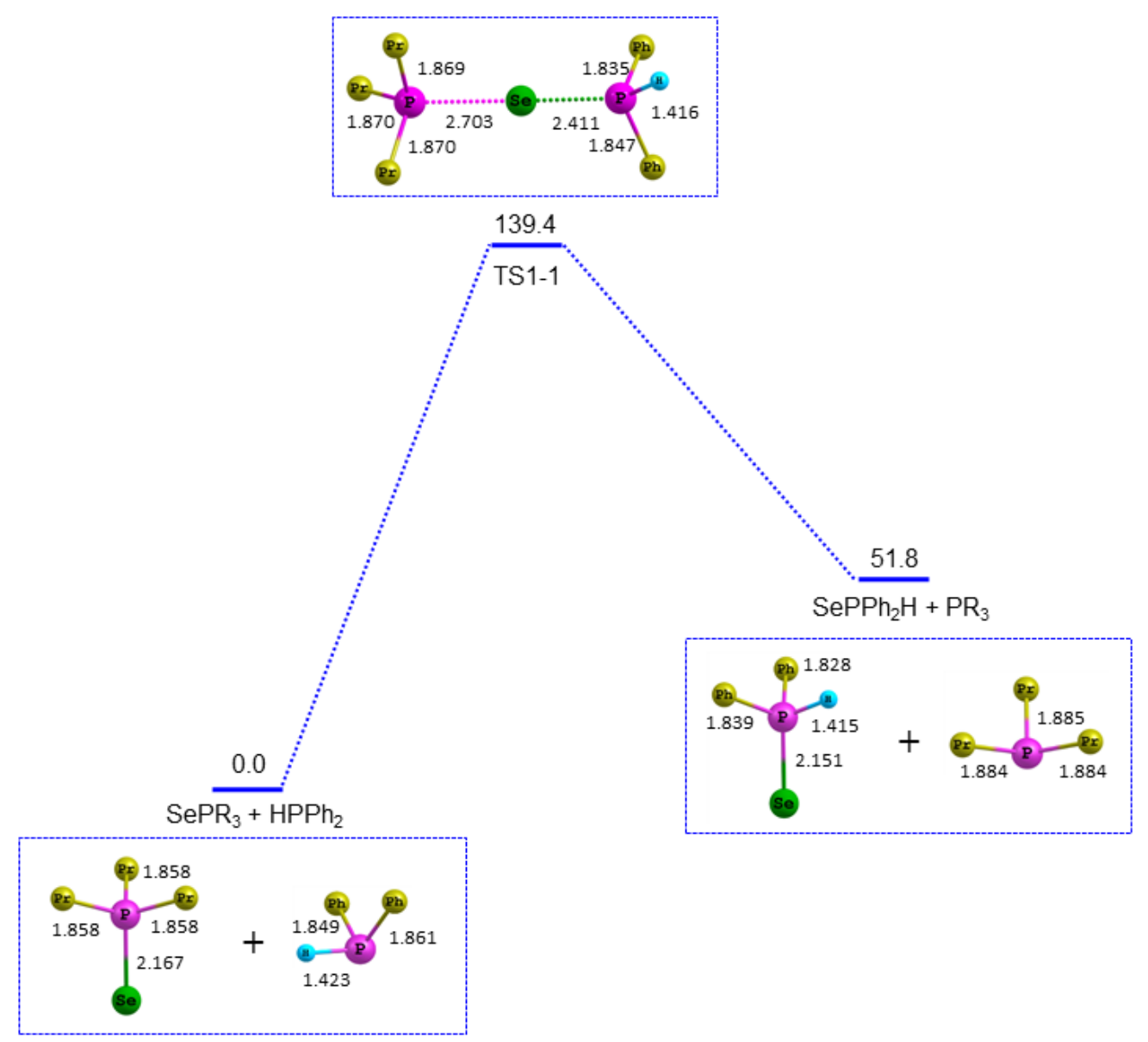

Figure S1b. The geometric structures of various species, and the schematic energy diagrams for the reaction $\mathrm{SePR}_{3}+\mathrm{HPPh}_{2}$ $\rightarrow \mathrm{SePPh}_{2} \mathrm{H}+\mathrm{PR}_{3}$. Bond lengths are reported in $\AA$. Relative Gibbs free energies $\left(G_{\mathrm{r}}\right)\left(\mathrm{kJ} \mathrm{mol}^{-1}\right)$ for the corresponding species relative to $\mathrm{SePR}_{3}+\mathrm{HPPh}_{2}$ at the M06//B3LYP/6-31++G(d, p), SDD level are shown. 


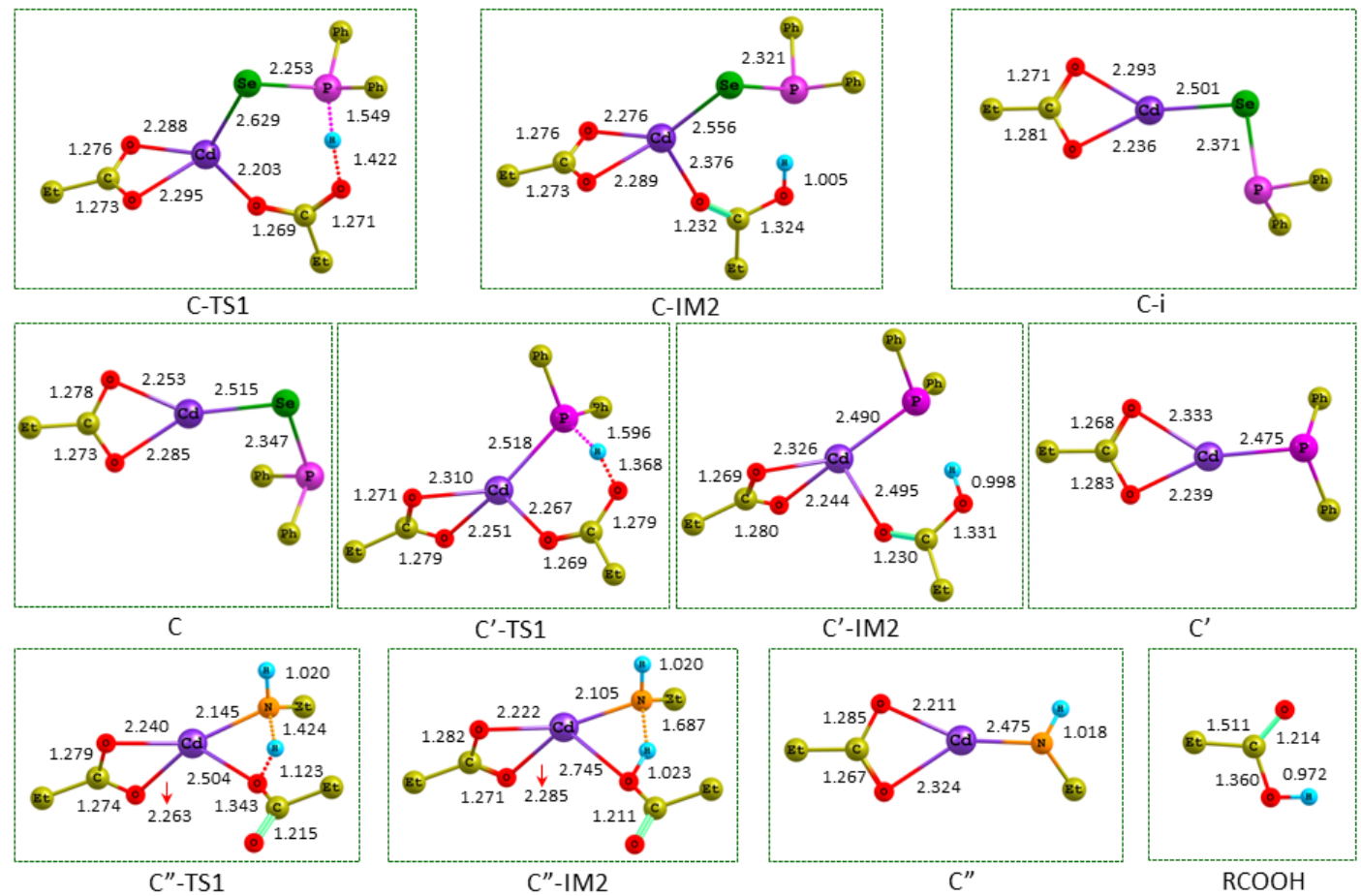

(a)

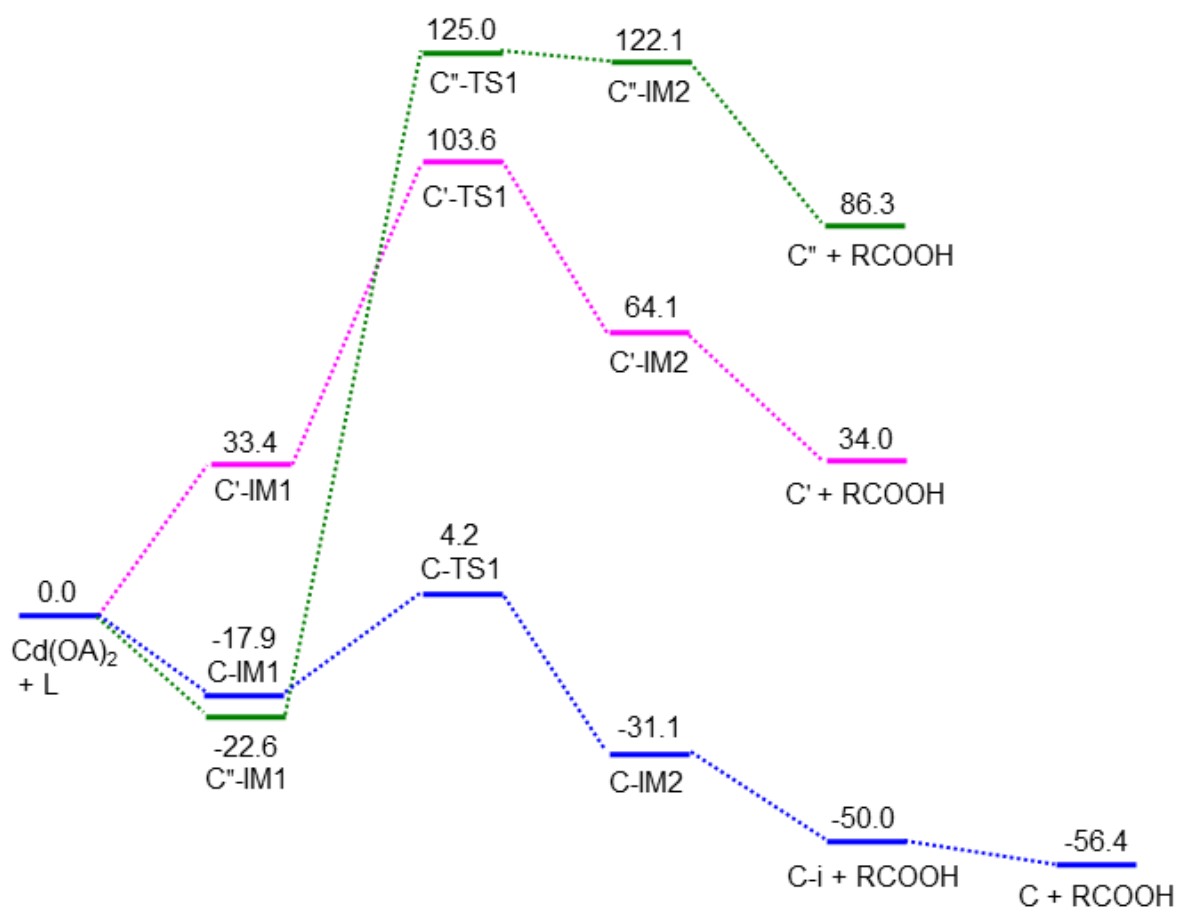

(b)

Figure S2. The geometric structures of various species (a), and the schematic energy diagrams (b) for the reaction (3) $\mathrm{Cd}(\mathrm{OA})_{2}+\mathrm{SePPh}_{2} \mathrm{H} \rightarrow \mathbf{C}+\mathrm{RCOOH},(4) \mathrm{Cd}(\mathrm{OA})_{2}+\mathrm{HPPh}_{2} \rightarrow \mathbf{C}^{\prime}+\mathrm{RCOOH}$, and (5) $\mathrm{Cd}(\mathrm{OA})_{2}+\mathrm{RNH}_{2} \rightarrow \mathbf{C}^{\prime \prime}+\mathrm{RCOOH}$ Bond lengths are reported in $\AA$. Relative Gibbs free energies $\left(G_{\mathrm{r}}\right)\left(\mathrm{kJ} \mathrm{mol}^{-1}\right)$ for the corresponding species relative to $\mathrm{Cd}(\mathrm{OA})_{2}$ $+\mathrm{L}\left(\mathrm{L}=\mathrm{SePPh}_{2} \mathrm{H}, \mathrm{HPPh}_{2}\right.$, and $\left.\mathrm{RNH}_{2}\right)$ at the M06//B3LYP/6-31++G(d, p), SDD level are shown. Blue, red, and green lines represent the reactions (3), (4), and (5), respectively. 
As shown in Figures S2a and S2b, for the reaction stage of $\mathrm{Cd}(\mathrm{OA})_{2}$ with $\mathrm{HPPh}_{2}$, to begin, the five-coordination complex $\left(\mathrm{HPPh}_{2}\right) \mathrm{Cd}(\mathrm{OA})_{2}\left(\mathbf{C}^{\prime}\right.$-IM1 $)$ is generated with the stabilization energy of $-33.4 \mathrm{~kJ} \mathrm{~mol}^{-1}$. Next, from $\mathbf{C}^{\prime}$-IM1, H-shift from $\mathrm{HPPh}_{2}$ to carboxylate occurs via a six-membered ring $\mathbf{C}^{\prime}$-TS1, resulting in the carboxylic acid molecular complex $\mathbf{C}^{\prime}$-IM2, with the energy barrier of $70.2 \mathrm{~kJ} \mathrm{~mol}^{-1}$ and the EHHP of $103.6 \mathrm{~kJ} \mathrm{~mol}^{-1}$ at $\mathbf{C}^{\prime}$-TS1. Then, $\mathbf{C}^{\prime}$-IM2 liberates the free carboxylic acid, leaving $\mathrm{RCOOCd}-\mathrm{PPh}_{2}\left(\mathbf{C}^{\prime}\right)$ behind, with the exoergicity of $30.1 \mathrm{~kJ} \mathrm{~mol}^{-1}$.

As shown in Figures S2a and S2b, for the reaction of $\mathrm{Cd}(\mathrm{OA})_{2}$ with $\mathrm{RNH}_{2}$, first, the five-coordination complex $\left(\mathrm{RNH}_{2}\right) \mathrm{Cd}(\mathrm{OA})_{2}\left(\mathbf{C}^{\prime \prime}\right.$-IM1 $)$ is engendered with the stabilization energy of $22.6 \mathrm{~kJ} \mathrm{~mol}^{-1}$. Second, from $\mathbf{C}^{\prime \prime}-\mathbf{I M 1}$,

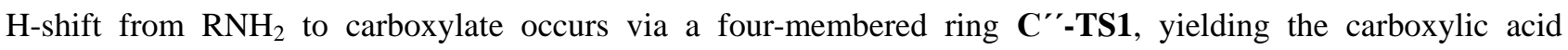
molecular complex $\mathbf{C}^{\prime \prime}$-IM2, with the energy barrier of $147.6 \mathrm{~kJ} \mathrm{~mol}^{-1}$ and the EHHP of $125.0 \mathrm{~kJ} \mathrm{~mol}^{-1}$ at $\mathbf{C}^{\prime \prime}$-TS1. Finally, $\mathbf{C}^{\prime \prime}$-IM2 releases the free carboxylic acid, leaving RCOOCd-NHR $\left(\mathbf{C}^{\prime \prime}\right)$ behind, with the exoergicity of $35.8 \mathrm{~kJ} \mathrm{~mol}^{-1}$. 

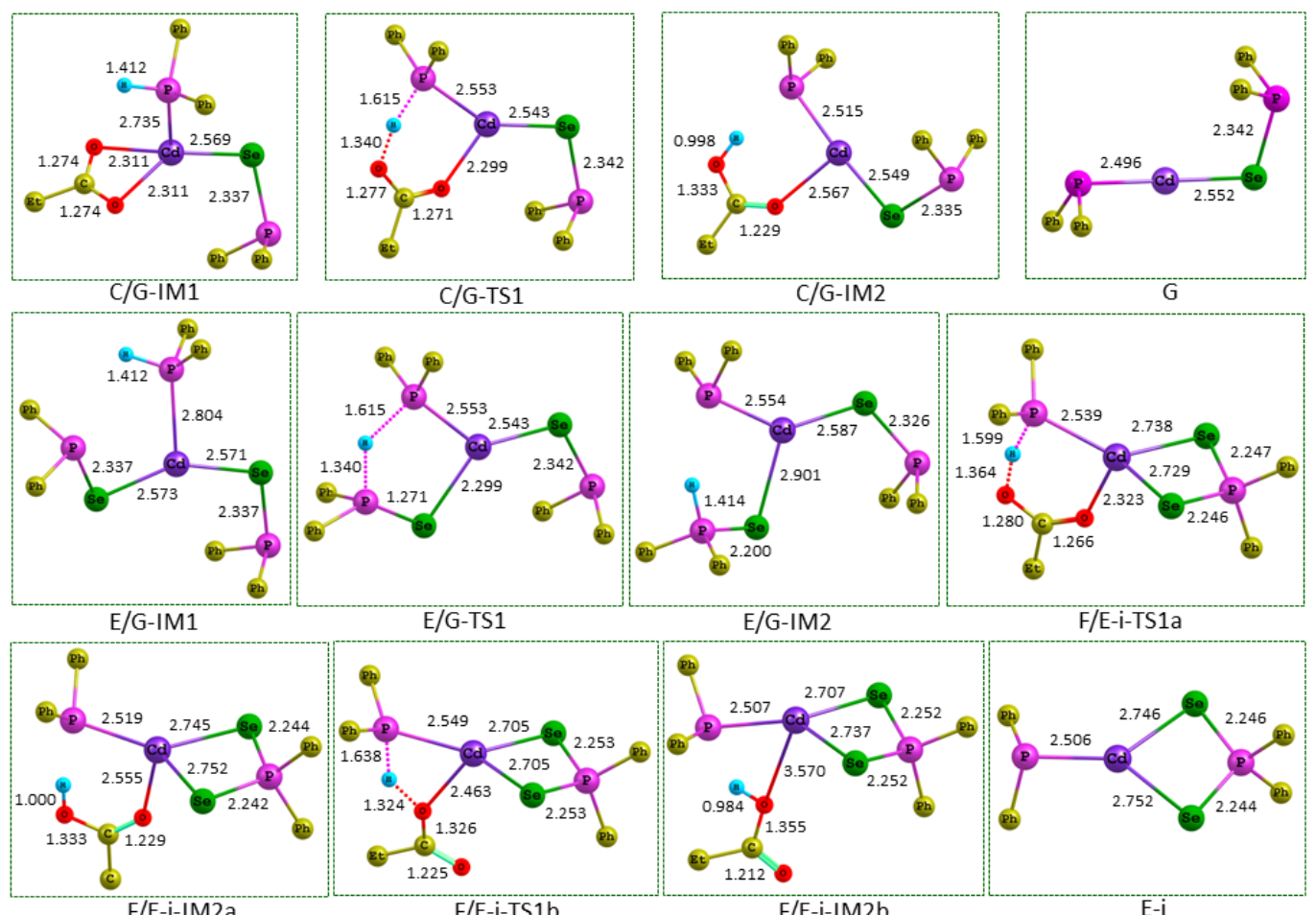

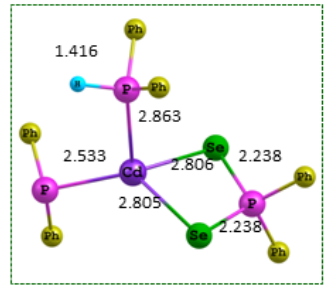

E-i/G-IM1

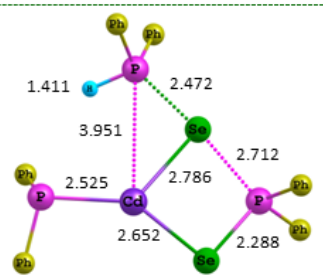

E-i/G-TS1

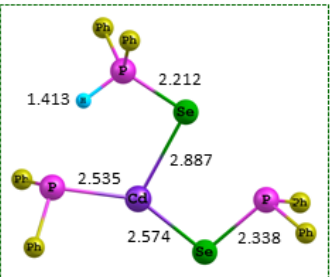

$\mathrm{E}-\mathrm{i} / \mathrm{G}-\mathrm{IM} 2$

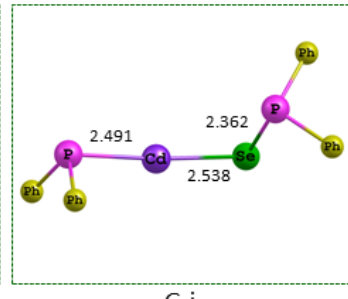

G-i

(a)

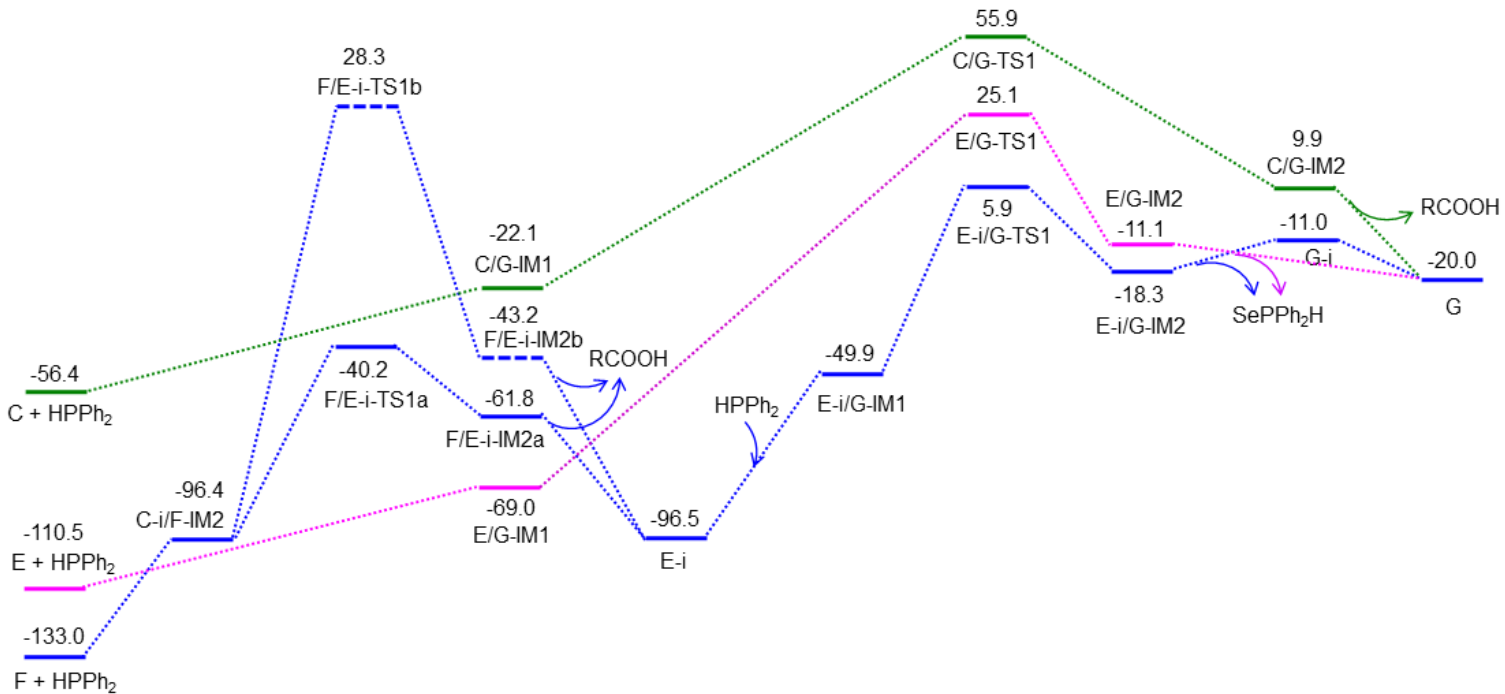

(b)

Figure S3. The geometric structures of various species (a), and the schematic energy diagrams (b) for the reaction $\mathrm{Cd}(\mathrm{OA})_{2}+$ $\mathrm{SePPh}_{2} \mathrm{H}+\mathrm{HPPh}_{2} \rightarrow \mathbf{G}+2 \mathrm{RCOOH}$ calculated at the M06//B3LYP/6-31++G(d, p), SDD level are shown. Bond lengths are reported in $\AA$. Relative Gibbs free energies $\left(G_{\mathrm{r}}\right)\left(\mathrm{kJ} \mathrm{mol}^{-1}\right)$ for the corresponding species plus RCOOH form $\mathbf{C}+\mathrm{HPPh}$ to $\mathbf{G}$, for the corresponding species plus $\mathrm{HPPh}_{2}+\mathrm{RCOOH}-\mathrm{SePPh}_{2} \mathrm{H}$ from $\mathbf{F}+\mathrm{HPPH}_{2}$ to $\mathbf{E}-\mathbf{i}$, and for the corresponding species plus $2 \mathrm{RCOOH}-\mathrm{SePPh}_{2} \mathrm{H}$ from $\mathbf{E}$ and $\mathbf{E}$-i to $\mathbf{G}$ are shown relative to $\mathrm{Cd}(\mathrm{OA})_{2}+\mathrm{SePPh}_{2} \mathrm{H}+\mathrm{HPPH}_{2}$. Green, red, and blue lines represent the reaction (8) $\mathbf{C}+\mathrm{HPPh}_{2} \rightarrow \mathbf{G}+\mathrm{RCOOH},(9) \mathbf{E}+\mathrm{HPPh}_{2} \rightarrow \mathbf{G}+\mathrm{SePPh}_{2} \mathrm{H}$, and (10) $\mathbf{F}+2 \mathrm{HPPh}_{2} \rightarrow \mathbf{G}+\mathrm{RCOOH}$ $+\mathrm{SePPh}_{2} \mathrm{H}$, respectively. 
As shown in Figures S3a and S3b, for the reaction of RCOOCdSe- $\mathrm{PPh}_{2}(\mathbf{C})$ with $\mathrm{HPPh}_{2}$, in the beginning, when the $\mathrm{P}$ atom of $\mathrm{HPPh}_{2}$ interacts with the central $\mathrm{Cd}$ atom of $\mathbf{C}$, a four-coordination complex $\mathbf{C} / \mathbf{G}-\mathbf{I M} 1$ is formed. From C/G-IM1, H-shift from $\mathrm{HPPh}_{2}$ to carboxylate occurs via a six-membered ring C/G-TS1, leading to a carboxylic acid molecular complex C/G-IM2. Then, C/G-IM2 releases the carboxylic acid, leaving $\mathrm{Ph}_{2} \mathrm{P}-\mathrm{CdSe}-\mathrm{PPh}_{2}(\mathbf{G})$ monomer behind. This reaction pathway involves the highest energy barrier (HEB) of 78.0 $\mathrm{kJ} \mathrm{mol}^{-1}$ at the reaction step of C/G-IM1 $\rightarrow$ C/G-TS1, the EHHP of $55.9 \mathrm{~kJ} \mathrm{~mol}^{-1}$ at C/G-TS1, and the endoergicity of $36.4 \mathrm{~kJ} \mathrm{~mol}^{-1}$.

As depicted in Figures S3a and S3b, for the reaction of $\mathrm{Ph}_{2} \mathrm{PSe}-\mathrm{Cd}-\mathrm{SePPh}_{2}(\mathbf{E})$ with $\mathrm{HPPh}_{2}$, initially, when the $\mathrm{P}$ atom of $\mathrm{HPPh}_{2}$ coordinates to the central Cd atom of $\mathbf{E}$, a three-coordination complex E/G-IM1 is generated. Next, from $\mathbf{E} / \mathbf{G}-\mathbf{I M 1}$, $\mathrm{H}$-shift from $\mathrm{HPPh}_{2}$ to $-\mathrm{PPh}_{2}$ group takes place via a five-membered ring $\mathbf{E} / \mathbf{G}-\mathbf{T S 1}$, resulting in a $\mathrm{SePPh}_{2} \mathrm{H}$ molecular complex E/G-IM2. After that, E/G-IM2 sets the $\mathrm{SePPh}_{2} \mathrm{H}$ free, leaving $\mathrm{Ph}_{2} \mathrm{P}-\mathrm{CdSe}-\mathrm{PPh}_{2}(\mathbf{G})$ monomer behind. This reaction pathway includes the HEB of $94.1 \mathrm{~kJ} \mathrm{~mol}^{-1}$ at the reaction step of E/G-IM1 $\rightarrow$ E/G-TS1, the EHHP of $25.1 \mathrm{~kJ} \mathrm{~mol}^{-1}$ at E/G-TS1, and the endoergicity of $90.5 \mathrm{~kJ} \mathrm{~mol}^{-1}$. 


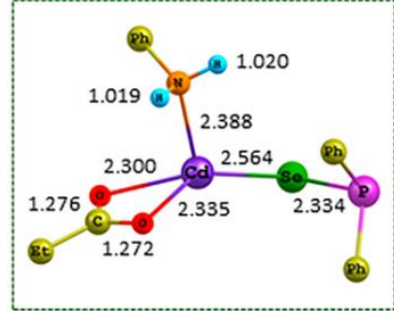

C/H-IM1

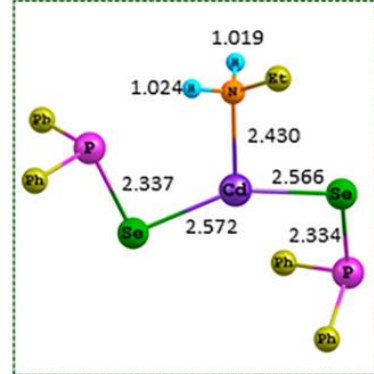

$$
\text { E/H-IM1 }
$$

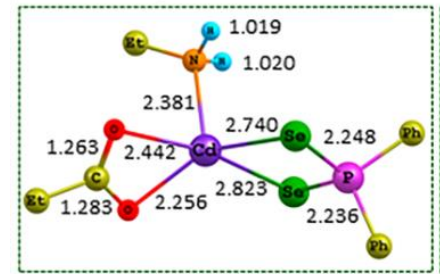

F/I-i-IM1

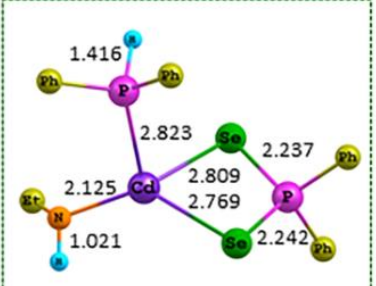

I-i/H-IM1

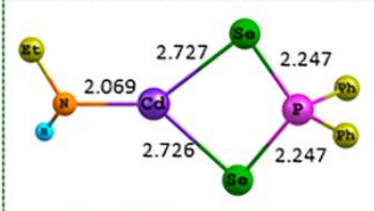

$\mathrm{I}-\mathrm{i}$

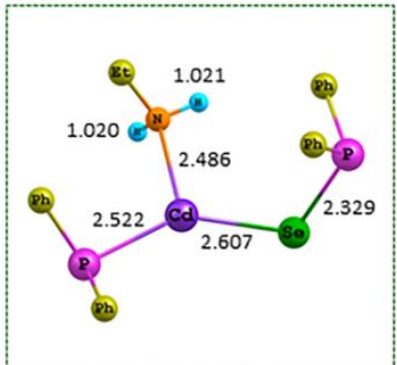

G/H-IM1

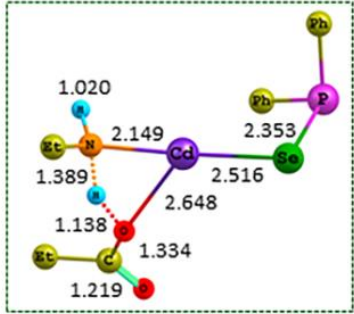

C/H-TS1

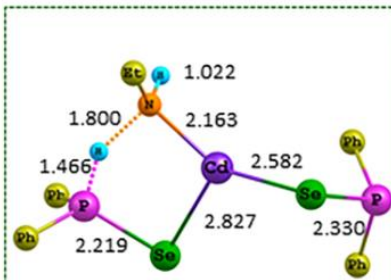

E/H-TS1

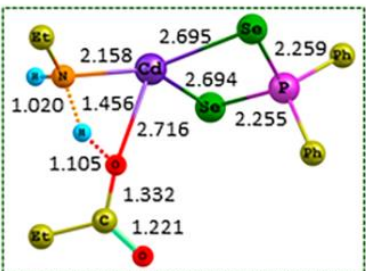

F/I-i-TS1

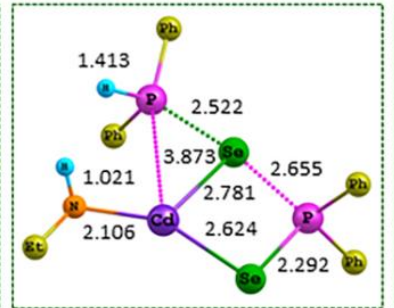

I-i/H-TS1

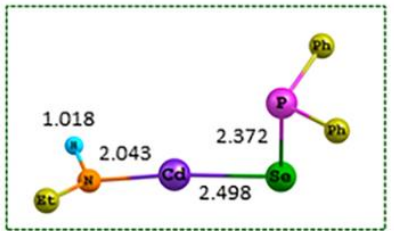

$\mathrm{H}-\mathrm{ii}$

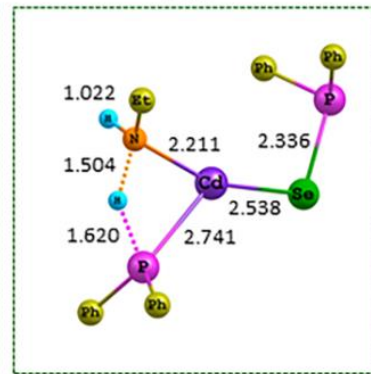

G/H-TS1

(a)

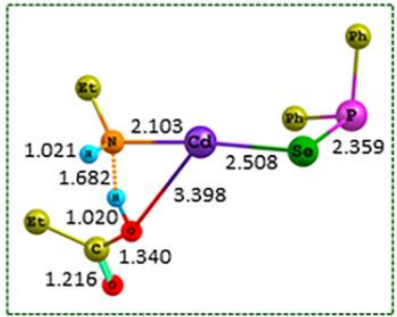

C/H-IM2

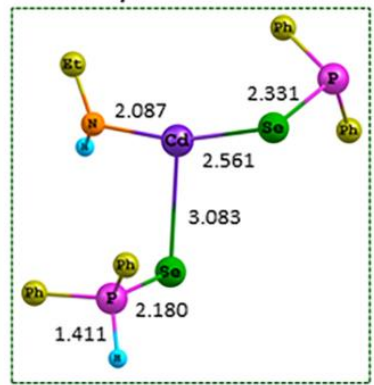

E/H-IM2
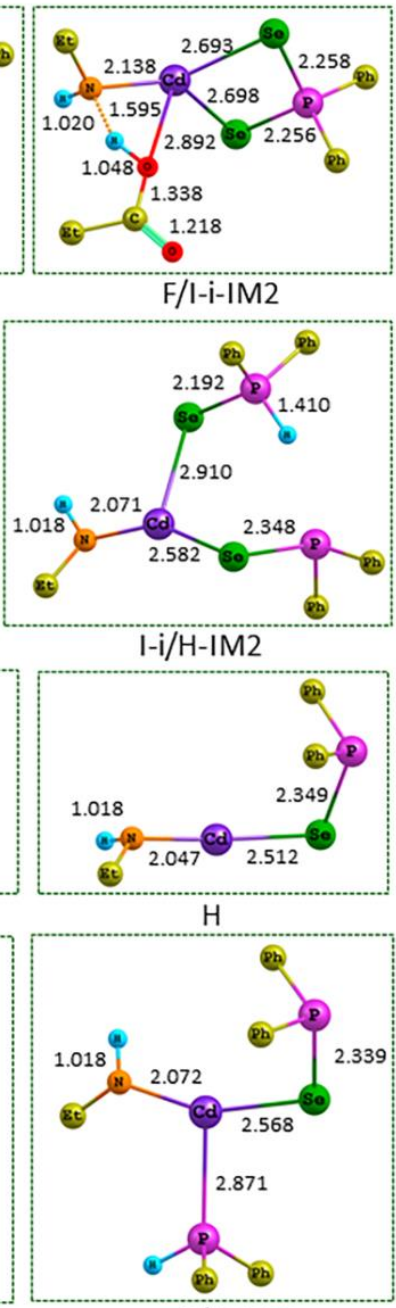

G/H-IM2 


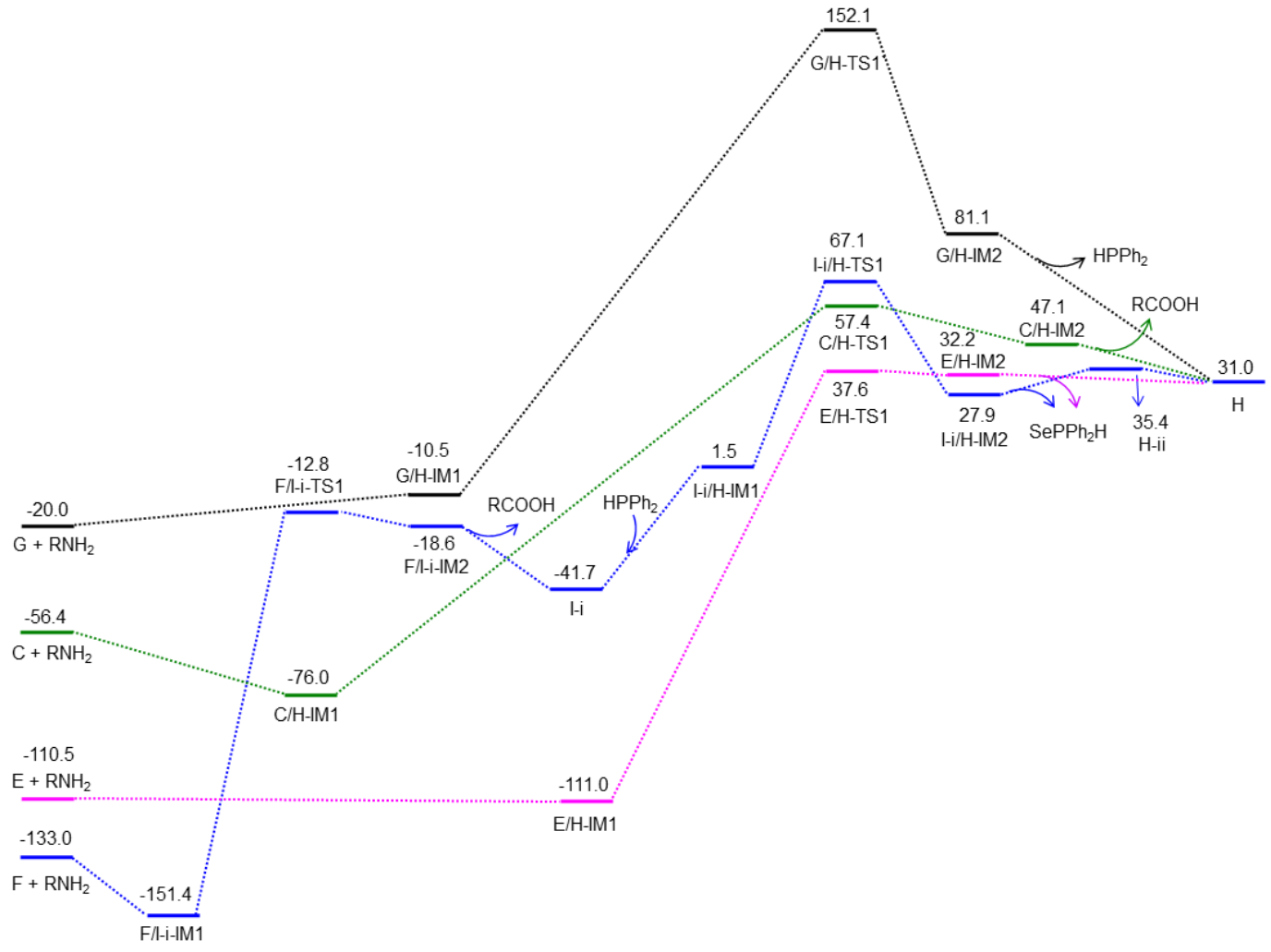

(b)

Figure S4. The geometric structures of various species (a), and the schematic energy diagrams (b) for the reaction $\mathrm{Cd}(\mathrm{OA})_{2}+$ $\mathrm{SePPh}_{2} \mathrm{H}+\mathrm{RNH}_{2} \rightarrow \mathbf{H}+2 \mathrm{RCOOH}$ calculated at the M06//B3LYP/6-31++G(d, p), SDD level are shown.. Bond lengths are reported in $\AA$. Relative Gibbs free energies $\left(G_{\mathrm{r}}\right)\left(\mathrm{kJ} \mathrm{mol}^{-1}\right)$ for the corresponding species plus RCOOH form $\mathbf{C}+\mathrm{RNH}_{2}$ to $\mathbf{H}$, for the corresponding species plus $\mathrm{HPPh}_{2}+\mathrm{RCOOH}-\mathrm{SePPh}_{2} \mathrm{H}$ from $\mathbf{F}+\mathrm{RNH}_{2}$ to $\mathbf{I}-\mathbf{i}$, for the corresponding species plus $2 \mathrm{RCOOH}-\mathrm{SePPh}_{2} \mathrm{H}$ from $\mathbf{I}-\mathbf{i}$ and $\mathbf{E}$ to $\mathbf{H}$, and for the corresponding species plus $2 \mathrm{RCOOH}-\mathrm{HPPh}_{2}$ from $\mathbf{G}+\mathrm{RNH}_{2}$ to $\mathbf{H}$ are shown relative to $\mathrm{Cd}(\mathrm{OA})_{2}+\mathrm{SePP}_{2} \mathrm{H}+\mathrm{RNH}_{2}$. Green, red, blue, and black lines represent the reaction (13) $\mathbf{C}+\mathrm{RNH}_{2} \rightarrow \mathbf{H}+$ $\mathrm{RCOOH}$, (14) $\mathbf{E}+\mathrm{RNH}_{2} \rightarrow \mathbf{H}+\mathrm{SePPh}_{2} \mathrm{H}$, (15) $\mathbf{F}+\mathrm{RNH}_{2}+\mathrm{HPPh}_{2} \rightarrow \mathbf{H}+\mathrm{RCOOH}+\mathrm{SePPh}_{2} \mathrm{H}$, and (16) $\mathbf{G}+\mathrm{RNH}_{2} \rightarrow \mathbf{H}+$ $\mathrm{HPPh}_{2}$, respectively.

As shown in Figures S4a and S4b, for the reaction of RCOOCdSe- $\mathrm{PPh}_{2}(\mathbf{C})$ with $\mathrm{RNH}_{2}$, initially, when the N-end of $\mathrm{RNH}_{2}$ coordinates to the central $\mathrm{Cd}$ atom of $\mathbf{C}$, a four-coordination complex C/H-IM1 is generated. From C/H-IM1, H-shift from $\mathrm{RNH}_{2}$ to carboxylate takes places via a four-membered ring C/H-TS1, yielding a carboxylic acid molecular complex C/H-IM2. Next, C/H-IM2 sets the carboxylic acid, leaving RHN-CdSe- $\mathrm{PPh}_{2}(\mathbf{H})$ monomer behind. This reaction pathway includes the highest energy barrier (HEB) of $133.4 \mathrm{~kJ} \mathrm{~mol}^{-1}$ at the reaction step of C/H-IM1 $\rightarrow$ C/H-TS1, the EHHP of 57.4 $\mathrm{kJ} \mathrm{mol}^{-1}$ at $\mathbf{C} / \mathbf{H}$-TS1, and the endoergicity of $87.4 \mathrm{~kJ} \mathrm{~mol}^{-1}$.

As depicted in Figures S4a and S4b, for the reaction of $\mathrm{Ph}_{2} \mathrm{P}-\mathrm{CdSe}-\mathrm{PPh}_{2}(\mathbf{G})$ with $\mathrm{RNH}_{2}$, initially, when the N-end of $\mathrm{RNH}_{2}$ interacts with the central $\mathrm{Cd}$ atom of $\mathbf{G}$, a three-coordination complex G/H-IM1 is generated. Next, from G/H-IM1, H-shift from $\mathrm{RNH}_{2}$ to $-\mathrm{PPh}_{2}$ group takes place via a four-membered ring G/H-TS1, resulting in a $\mathrm{HPPh}_{2}$ molecular complex G/H-IM2. Lastly, G/H-IM2 releases the free $\mathrm{HPPh}_{2}$, leaving RHN-CdSe- $\mathrm{PPh}_{2}(\mathbf{H})$ monomer behind. This reaction pathway comprises the HEB of $162.6 \mathrm{~kJ} \mathrm{~mol}^{-1}$ at the reaction step of G/H-IM1 $\rightarrow$ G/H-TS1, the EHHP of $152.1 \mathrm{~kJ} \mathrm{~mol}^{-1}$ at G/H-TS1, and the endoergicity of $141.5 \mathrm{~kJ} \mathrm{~mol}^{-1}$. 

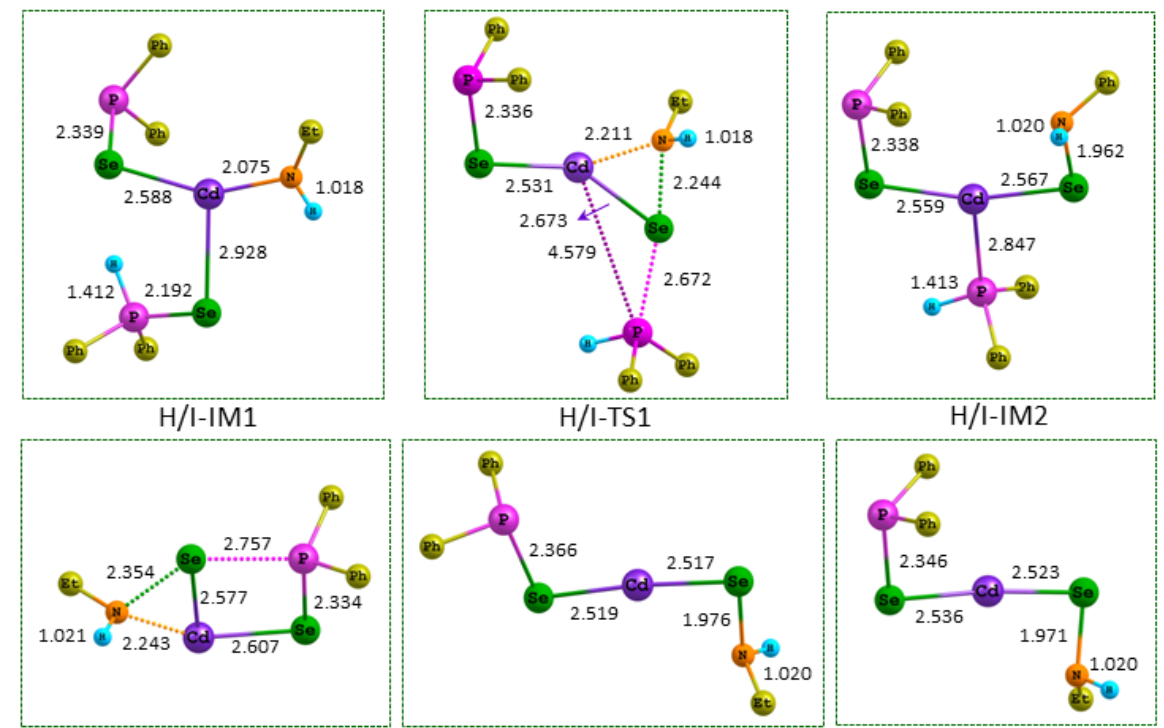

H/I-IM2

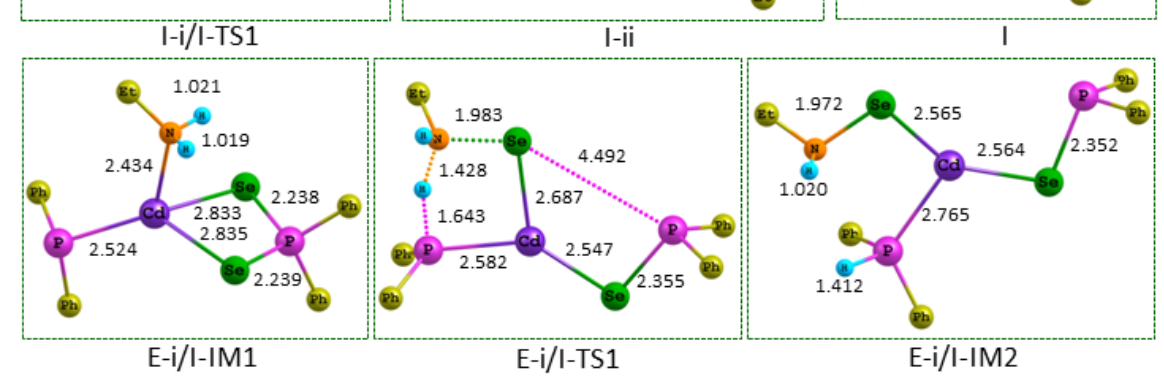

(a)

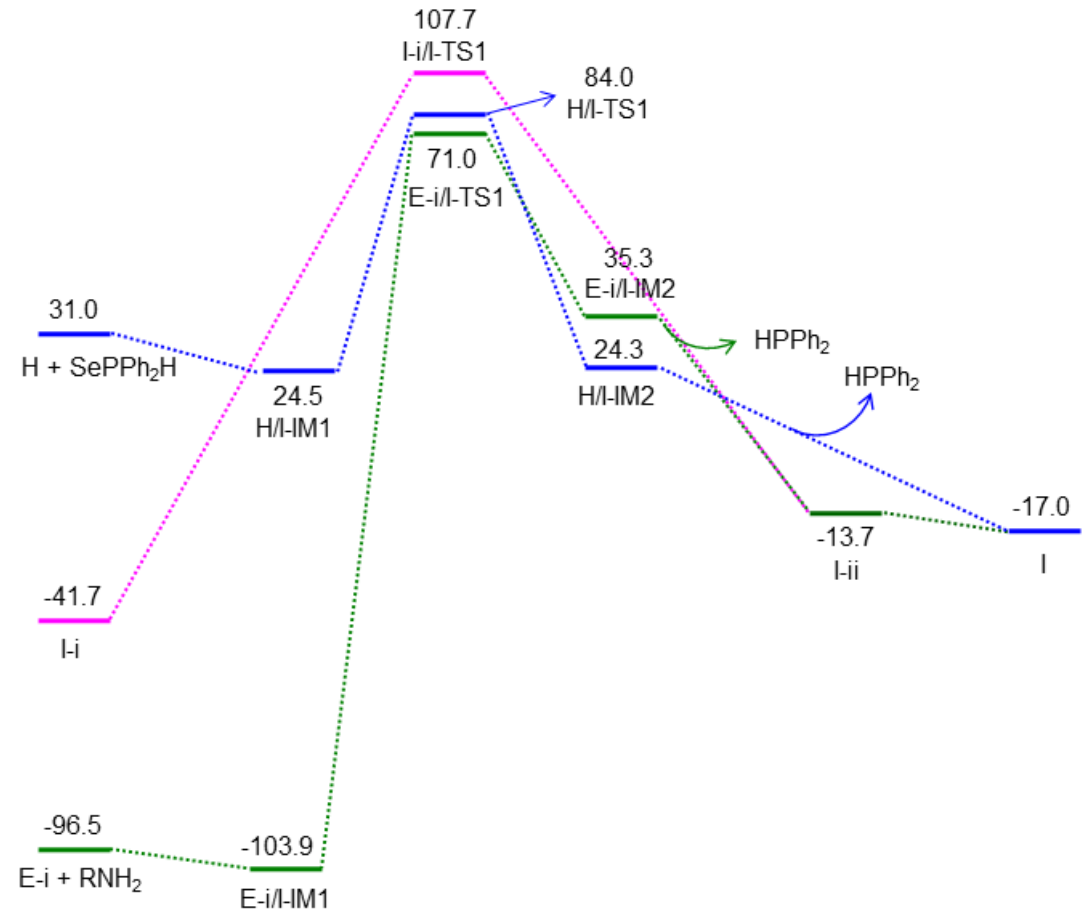

(b)

Figure S5. The geometric structures of various species (a), and the schematic energy diagrams (b) for the reaction $\mathrm{Cd}(\mathrm{OA})_{2}+$ $2 \mathrm{SePPh}_{2} \mathrm{H}+\mathrm{RNH}_{2} \rightarrow \mathbf{I}+\mathrm{HPPh}_{2}+2 \mathrm{RCOOH}$ calculated at the M06//B3LYP/6-31++G(d, p), SDD level are shown. Bond lengths are reported in $\AA$. Relative Gibbs free energies $\left(G_{\mathrm{r}}\right)\left(\mathrm{kJ} \mathrm{mol}^{-1}\right)$ for the corresponding species plus $2 \mathrm{RCOOH}$ from $\mathbf{H}$ $+\mathrm{SePPh}_{2} \mathrm{H}$ and $\mathbf{E}-\mathbf{i}+\mathrm{RNH}_{2}$ to $\mathbf{I}$, and for the corresponding species plus $\mathrm{HPPh}_{2}+2 \mathrm{RCOOH}$ from $\mathbf{I}-\mathbf{i}$ to $\mathbf{I}$ are shown relative to $\mathrm{Cd}(\mathrm{OA})_{2}+2 \mathrm{SePPh}_{2} \mathrm{H}+\mathrm{RNH}_{2}$. Blue, red, and Green lines represent the reaction (21) $\mathbf{H}+\mathrm{SePPh}_{2} \mathrm{H} \rightarrow \mathbf{I}+\mathrm{HPPh}_{2}$, (22) I-i $\rightarrow \mathbf{I}$, and (23) $\mathbf{E}-\mathbf{i}+\mathrm{RNH}_{2} \rightarrow \mathbf{I}+\mathrm{HPPh}_{2}$, respectively. 
As shown in Figures S5a and S5b, for the reaction stage (21) of $\mathrm{RHN}-\mathrm{CdSe}-\mathrm{PPh}_{2}(\mathbf{H})$ with $\mathrm{SePPh}_{2} \mathrm{H}$, in the beginning, when the Se-end of $\mathrm{SePPh}_{2} \mathrm{H}$ interacts with the central $\mathrm{Cd}$ atom of $\mathbf{H}$, a three-coordination complex H/I-IM1 is formed. Then, from H/I-IM1, Se exchange takes place through the synergistic $\mathrm{Se}=\mathrm{P}$ bond cleavage of $\mathrm{SePPh}_{2} \mathrm{H}$ moiety, Cd-N bond cleavage, and Se-N bond formation via a four-membered plane-like H/I-TS1, resulting in a $\mathrm{HPPh}_{2}$ molecular complex H/I-IM2. Lastly, H/I-IM2 sets the $\mathrm{HPPh}_{2}$ free, leaving $\mathrm{Ph}_{2} \mathrm{PSe}-\mathrm{CdSe}-\mathrm{NHR}$ (I) monomer behind. Wherein, $\mathrm{SePPh}_{2} \mathrm{H}$ serves as Se source for the precursor formation.

For the $\mathbf{I}$ formation from $\mathbf{E}$, this reaction pathway RP-CEHI includes the HEB of $148.6 \mathrm{~kJ} \mathrm{~mol}^{-1}$ at the reaction step of E/H-IM1 $\rightarrow$ E/H-TS1, the EHHP of $84.0 \mathrm{~kJ} \mathrm{~mol}^{-1}$ at H/I-TS1, and the endoergicity of $93.5 \mathrm{~kJ} \mathrm{~mol}^{-1}$, together with the reaction (14) of $\mathbf{E}+\mathrm{RNH}_{2} \rightarrow \mathbf{H}+\mathrm{SePPh}_{2} \mathrm{H}$.

As shown in Figures S5a and S5b, for the isomerization (22) of RHN-Cd-Se $\mathrm{PPh}_{2}(\mathbf{I}-\mathbf{i})$, from I-i, Se exchange occurs through the synchronous Se-P bond cleavage of $\mathrm{Se}_{2} \mathrm{PPh}_{2} \mathrm{H}$ moiety, Cd-N bond cleavage, and Se-N bond formation via a five-membered envelope-like I-i/I-TS1, producing an isomer trans- $\mathrm{Ph}_{2} \mathrm{PSe}-\mathrm{CdSe}-\mathrm{NHR}$ (I-ii). Last, I-ii can easily isomerize to $\mathrm{Ph}_{2} \mathrm{PSe}-\mathrm{CdSe}-\mathrm{NHR}(\mathrm{I})$, with the $\mathrm{Se}-\mathrm{P}$ single bond rotation.

For the $\mathbf{I}$ formation from $\mathbf{F}$, this reaction pathway RP-CFI-iI involves the HEB of $149.4 \mathrm{~kJ} \mathrm{~mol}^{-1}$ at the reaction step of $\mathbf{I}-\mathbf{i} \rightarrow \mathbf{I}-\mathbf{i} / \mathbf{I}-\mathbf{T S 1}$, the EHHP of $107.7 \mathrm{~kJ} \mathrm{~mol}^{-1}$ at $\mathbf{I}-\mathbf{i} / \mathbf{I}-\mathbf{T S 1}$, and the endoergicity of $116.0 \mathrm{~kJ} \mathrm{~mol}^{-1}$, together with the reaction (17) of $\mathbf{F}+\mathrm{RNH}_{2} \rightarrow \mathbf{I}-\mathbf{i}+\mathrm{RCOOH}$. 


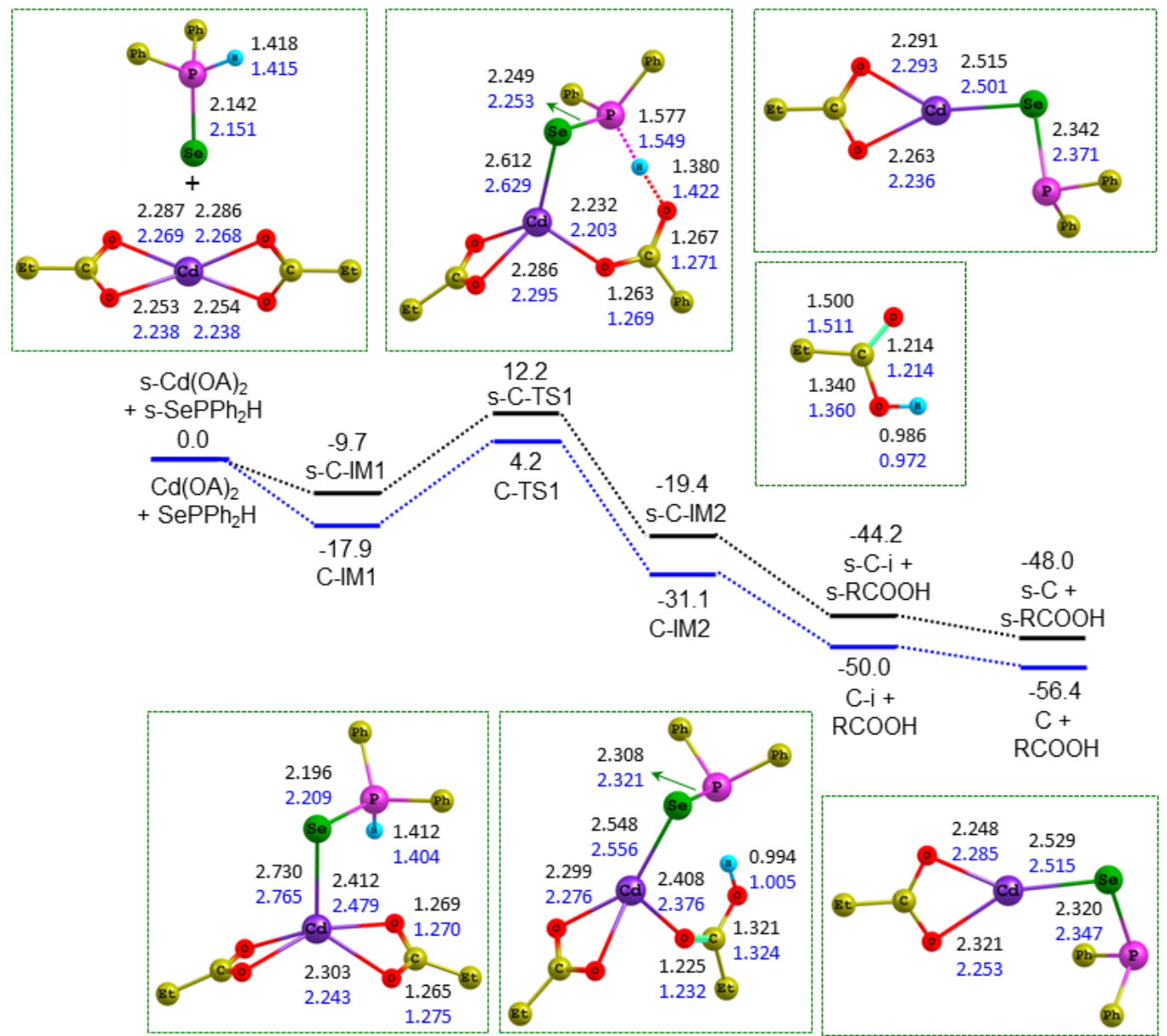

Figure S6. The geometric structures and the schematic energy diagrams for the reaction $\mathrm{Cd}(\mathrm{OA})_{2}+\mathrm{SePPh}_{2} \mathrm{H} \rightarrow \mathbf{C}+\mathrm{RCOOH}$ calculated at the M06/6-31++G(d, p), SDD level in the ODE media under room temperature and atmospheric pressure (298.15 $\mathrm{K}$ and $1 \mathrm{~atm})$. Relative Gibbs free energies $\left(G_{\mathrm{r}}\right)\left(\mathrm{kJ} \mathrm{mol}^{-1}\right)$ for the corresponding species relative to $\mathrm{Cd}(\mathrm{OA})_{2}+\mathrm{SePPh}_{2} \mathrm{H}$ are shown. Bond lengths are reported in $\AA$. Black and blue lines represent the level of M06/6-31++G(d, p), SDD and M06//B3LYP/6-31++G(d, p), SDD, respectively. 
Table S1. The zero-point energies ( $Z P E$, hartree), thermal correction to Gibbs free energy $\left(G_{0}\right.$, hartree) of various species calculated at the B3LYP/6-31++G(d, p), SDD level in the gas phase under atmospheric pressure and room temperature (298.15 K and 1 atm). Polarizable continuum model correction energies (PCM-E, hartree), total energies ( $E_{\mathrm{c}}$, hartree) corrected by $Z P E$, sum of electronic and thermal free energies $\left(G_{\mathrm{c}}\right.$, hartree) corrected by $G_{0}$, relative energies $\left(E_{\mathrm{r}}, \mathrm{kJ} \mathrm{mol}^{-1}\right)$ and relative Gibbs free energies $\left(G_{\mathrm{r}}, \mathrm{kJ} \mathrm{mol}^{-1}\right)$ to $\mathrm{Cd}(\mathrm{OA})_{2}+n \mathrm{~L}\left(\mathrm{~L}=\mathrm{SePR}_{3}, \mathrm{RNH}_{2}, \mathrm{SePPh}_{2} \mathrm{H}, \mathrm{PR}_{3}\right.$, and $\left.\mathrm{HPPh}_{2} ; n=1 \sim 2\right)$ for all the species in the reaction of $\mathrm{Cd}(\mathrm{OA})_{2}+n \mathrm{~L} \rightarrow$ $\mathrm{L}_{n} \mathrm{Cd}(\mathrm{OA})_{2}$ calculated at the M06//B3LYP/6-31++G(d, p), SDD level in 1-octadecene solution under room temperature and atmospheric pressure $(298.15 \mathrm{~K}$ and $1 \mathrm{~atm})$.

M06//B3LYP/6-31++G(d, p), SDD

Species

(298.15 $\mathrm{K}$ and $1 \mathrm{~atm})$

\begin{tabular}{|c|c|c|c|c|c|c|c|}
\hline \multirow{2}{*}{ Species } & \\
\hline & $Z P E$ & $G_{0}$ & $P C M-E$ & $\begin{array}{c}E_{\mathrm{c}}(P C M-E+ \\
Z P E)\end{array}$ & $E_{\mathrm{r}}$ & $G_{\mathrm{c}}\left(P C M-E+G_{0}\right)$ & $G_{\mathrm{r}}$ \\
\hline $\mathrm{SePR}_{3}$ & 0.28681 & 0.24187 & -706.02637 & -705.73957 & & -705.78450 & \\
\hline $\mathrm{RNH}_{2}$ & 0.09263 & 0.06713 & -135.08775 & -134.99512 & & -135.02062 & \\
\hline $\mathrm{SePPh}_{2} \mathrm{H}$ & 0.19343 & 0.15007 & -814.22030 & -814.02687 & & -814.07023 & \\
\hline $\mathrm{PR}_{3}$ & 0.28422 & 0.24265 & -696.66425 & -696.38003 & & -696.42160 & \\
\hline $\mathrm{HPPh}_{2}$ & 0.19113 & 0.15188 & -804.87857 & -804.68745 & & -804.72669 & \\
\hline $\mathrm{Cd}(\mathrm{OA})_{2}$ & 0.15896 & 0.11333 & -703.16954 & -703.01058 & & -703.05621 & \\
\hline $\mathrm{SePR}_{3}+\mathrm{Cd}(\mathrm{OA})_{2}$ & 0.44577 & 0.35520 & -1409.19591 & -1408.75014 & 0.0 & -1408.84071 & 0.0 \\
\hline$\left(\mathrm{SePR}_{3}\right) \mathrm{Cd}(\mathrm{OA})_{2}$ & 0.44681 & 0.37459 & -1409.22945 & -1408.78264 & -85.3 & -1408.85486 & -37.2 \\
\hline $\mathrm{RNH}_{2}+\mathrm{Cd}(\mathrm{OA})_{2}$ & 0.25159 & 0.18045 & -838.25729 & -838.00570 & 0.0 & -838.07684 & 0.0 \\
\hline$\left(\mathrm{RNH}_{2}\right) \mathrm{Cd}(\mathrm{OA})_{2}$ & 0.25398 & 0.19822 & -838.28365 & -838.02967 & -62.9 & -838.08543 & -22.6 \\
\hline $\mathrm{SePPh}_{2} \mathrm{H}+\mathrm{Cd}(\mathrm{OA})_{2}$ & 0.35239 & 0.26339 & -1517.38984 & -1517.03744 & 0.0 & -1517.12644 & 0.0 \\
\hline$\left(\mathrm{SePPh}_{2} \mathrm{H}\right) \mathrm{Cd}(\mathrm{OA})_{2}$ & 0.35320 & 0.28247 & -1517.41571 & -1517.06251 & -65.8 & -1517.13325 & -17.9 \\
\hline $\mathrm{PR}_{3}+\mathrm{Cd}(\mathrm{OA})_{2}$ & 0.44318 & 0.35597 & -1399.83378 & -1399.39060 & 0.0 & -1399.47781 & 0.0 \\
\hline$\left(\mathrm{PR}_{3}\right) \mathrm{Cd}(\mathrm{OA})_{2}$ & 0.44452 & 0.37318 & -1399.85552 & -1399.41100 & -53.6 & -1399.48234 & -11.9 \\
\hline $\mathrm{HPPh}_{2}+\mathrm{Cd}(\mathrm{OA})_{2}$ & 0.35009 & 0.26521 & -1508.04811 & -1507.69802 & 0.0 & -1507.78290 & 0.0 \\
\hline$\left(\mathrm{HPPh}_{2}\right) \mathrm{Cd}(\mathrm{OA})_{2}$ & 0.35143 & 0.28474 & -1508.05490 & -1507.70346 & -14.3 & -1507.77016 & 33.4 \\
\hline $2 \mathrm{SePR}_{3}+\mathrm{Cd}(\mathrm{OA})_{2}$ & 0.73258 & 0.59707 & -2115.22229 & -2114.48971 & 0.0 & -2114.62522 & 0.0 \\
\hline$\left(\mathrm{SePR}_{3}\right)_{2} \mathrm{Cd}(\mathrm{OA})_{2}$ & 0.73463 & 0.63899 & -2115.28366 & -2114.54903 & -155.7 & -2114.64467 & -51.1 \\
\hline $2 \mathrm{RNH}_{2}+\mathrm{Cd}(\mathrm{OA})_{2}$ & 0.34422 & 0.24758 & -973.34504 & -973.00082 & 0.0 & -973.09746 & 0.0 \\
\hline$\left(\mathrm{RNH}_{2}\right)_{2} \mathrm{Cd}(\mathrm{OA})_{2}$ & 0.34943 & 0.28819 & -973.38793 & -973.03850 & -98.9 & -973.09974 & -6.0 \\
\hline $2 \mathrm{SePPh}_{2} \mathrm{H}+\mathrm{Cd}(\mathrm{OA})_{2}$ & 0.54582 & 0.41346 & -2331.61013 & -2331.06431 & 0.0 & -2331.19667 & 0.0 \\
\hline$\left(\mathrm{SePPh}_{2} \mathrm{H}\right)_{2} \mathrm{Cd}(\mathrm{OA})_{2}$ & 0.54723 & 0.45443 & -2331.64770 & -2331.10048 & -95.0 & -2331.19327 & 8.9 \\
\hline $2 \mathrm{PR}_{3}+\mathrm{Cd}(\mathrm{OA})_{2}$ & 0.72740 & 0.59862 & -2096.49803 & -2095.77063 & 0.0 & -2095.89941 & 0.0 \\
\hline$\left(\mathrm{PR}_{3}\right)_{2} \mathrm{Cd}(\mathrm{OA})_{2}$ & 0.73095 & 0.64043 & -2096.53926 & -2095.80831 & -98.9 & -2095.89884 & 1.5 \\
\hline $2 \mathrm{HPPh}_{2}+\mathrm{Cd}(\mathrm{OA})_{2}$ & 0.54122 & 0.41709 & -2312.92668 & -2312.38547 & 0.0 & -2312.50959 & 0.0 \\
\hline$\left(\mathrm{HPPh}_{2}\right)_{2} \mathrm{Cd}(\mathrm{OA})_{2}$ & 0.54372 & 0.45988 & -2312.93939 & -2312.39567 & -26.8 & -2312.47951 & 79.0 \\
\hline
\end{tabular}


Table S2. The zero-point energies ( $Z P E$, hartree), thermal correction to Gibbs free energy $\left(G_{0}\right.$, hartree) of various species calculated at the B3LYP/6-31++G(d, p), SDD level in the gas phase under atmospheric pressure and room temperature (298.15 K and 1 atm). Polarizable continuum model correction energies (PCM-E, hartree), total energies ( $E_{\mathrm{c}}$, hartree) corrected by $Z P E$, sum of electronic and thermal free energies $\left(G_{\mathrm{c}}\right.$, hartree) corrected by $G_{0}$, relative energies $\left(E_{\mathrm{r}}, \mathrm{kJ} \mathrm{mol}^{-1}\right)$ and relative Gibbs free energies $\left(G_{\mathrm{r}}, \mathrm{kJ}^{\mathrm{mol}}{ }^{-1}\right)$ to $\mathrm{Cd}(\mathrm{OA})_{2}+\mathrm{L}\left(\mathrm{L}=\mathrm{SePPh}_{2} \mathrm{H}, \mathrm{HPPh}_{2}, \mathrm{RNH}_{2}\right)$ for all the species in the reaction of $\mathrm{Cd}(\mathrm{OA})_{2}+\mathrm{SePPh}_{2} \mathrm{H} \rightarrow \mathbf{C}+\mathrm{RCOOH}, \mathrm{Cd}(\mathrm{OA})_{2}+$ $\mathrm{HPPh}_{2} \rightarrow \mathbf{C}^{\prime}+\mathrm{RCOOH}$, and $\mathrm{Cd}(\mathrm{OA})_{2}+\mathrm{RNH}_{2} \rightarrow \mathbf{C}^{\prime \prime}+\mathrm{RCOOH}$ calculated at the M06//B3LYP/6-31++G(d, p), SDD level in 1-octadecene solution under room temperature and atmospheric pressure (298.15 K and $1 \mathrm{~atm})$.

M06//B3LYP/6-31++G(d, p), SDD

Species

(298.15 $\mathrm{K}$ and $1 \mathrm{~atm})$

\begin{tabular}{|c|c|c|c|c|c|c|c|}
\hline \multirow{2}{*}{ Species } & \\
\hline & $Z P E$ & $G_{0}$ & $P C M-E$ & $\begin{array}{c}E_{\mathrm{c}}(P C M-E+ \\
Z P E)\end{array}$ & $E_{\mathrm{r}}$ & $G_{\mathrm{c}}\left(P C M-E+G_{0}\right)$ & $G_{\mathrm{r}}$ \\
\hline $\mathrm{SePPh}_{2} \mathrm{H}$ & 0.19343 & 0.15007 & -814.22030 & -814.02687 & & -814.07023 & \\
\hline $\mathrm{HPPh}_{2}$ & 0.19113 & 0.15188 & -804.87857 & -804.68745 & & -804.72669 & \\
\hline $\mathrm{RNH}_{2}$ & 0.09263 & 0.06713 & -135.08775 & -134.99512 & & -135.02062 & \\
\hline $\mathrm{Cd}(\mathrm{OA})_{2}$ & 0.15896 & 0.11333 & -703.16954 & -703.01058 & & -703.05621 & \\
\hline $\mathrm{RCOOH}$ & 0.09058 & 0.06013 & -268.27275 & -268.18218 & & -268.21262 & \\
\hline $\mathrm{SePPh}_{2} \mathrm{H}+\mathrm{Cd}(\mathrm{OA})_{2}$ & 0.35239 & 0.26339 & -1517.38984 & -1517.03744 & 0.0 & -1517.12644 & 0.0 \\
\hline C-IM1 & 0.35320 & 0.28247 & -1517.41571 & -1517.06251 & -65.8 & -1517.13325 & -17.9 \\
\hline C-TS1 & 0.35003 & 0.27966 & -1517.40449 & -1517.05447 & -44.7 & -1517.12484 & 4.2 \\
\hline C-IM2 & 0.35466 & 0.28385 & -1517.42214 & -1517.06748 & -78.9 & -1517.13829 & -31.1 \\
\hline $\mathrm{C}-\mathrm{i}$ & 0.26351 & 0.20406 & -1249.13693 & -1248.87342 & & -1248.93286 & \\
\hline $\mathrm{C}-\mathrm{i}+\mathrm{RCOOH}$ & 0.35408 & 0.26420 & -1517.40968 & -1517.05560 & -47.7 & -1517.14548 & -50.0 \\
\hline $\mathrm{C}$ & 0.26354 & 0.20472 & -1249.14001 & -1248.87647 & & -1248.93529 & \\
\hline $\mathrm{C}+\mathrm{RCOOH}$ & 0.35412 & 0.26486 & -1517.41276 & -1517.05865 & -55.7 & -1517.14791 & -56.4 \\
\hline $\mathrm{HPPh}_{2}+\mathrm{Cd}(\mathrm{OA})_{2}$ & 0.35009 & 0.26521 & -1508.04811 & -1507.69802 & 0.0 & -1507.78290 & 0.0 \\
\hline$C^{\prime}-\mathrm{IM} 1$ & 0.35143 & 0.28474 & -1508.05490 & -1507.70346 & -14.3 & -1507.77016 & 33.4 \\
\hline $\mathrm{C}^{\prime}-\mathrm{TS} 1$ & 0.34809 & 0.28269 & -1508.02614 & -1507.67805 & 52.4 & -1507.74345 & 103.6 \\
\hline$C^{\prime}-\mathrm{IM} 2$ & 0.35338 & 0.28580 & -1508.04428 & -1507.69090 & 18.7 & -1507.75848 & 64.1 \\
\hline$C^{\prime}$ & 0.26214 & 0.20651 & -1239.76385 & -1239.50171 & & -1239.55734 & \\
\hline $\mathrm{C}^{\prime}+\mathrm{RCOOH}$ & 0.35272 & 0.26664 & -1508.03660 & -1507.68388 & 37.1 & -1507.76996 & 34.0 \\
\hline $\mathrm{RNH}_{2}+\mathrm{Cd}(\mathrm{OA})_{2}$ & 0.25159 & 0.18045 & -838.25729 & -838.00570 & 0.0 & -838.07684 & 0.0 \\
\hline $\mathrm{C}^{\prime \prime}-\mathrm{IM} 1$ & 0.25398 & 0.19822 & -838.28365 & -838.02967 & -62.9 & -838.08543 & -22.6 \\
\hline $\mathrm{C}^{\prime \prime}-\mathrm{TS} 1$ & 0.24905 & 0.19528 & -838.22450 & -837.97545 & 79.4 & -838.02922 & 125.0 \\
\hline $\mathrm{C}^{\prime \prime}-\mathrm{IM} 2$ & 0.25206 & 0.19646 & -838.22681 & -837.97474 & 81.3 & -838.03035 & 122.1 \\
\hline $\mathrm{C}^{\prime \prime}$ & 0.16094 & 0.11845 & -569.94980 & -569.78886 & & -569.83135 & \\
\hline $\mathrm{C}^{\prime \prime}+\mathrm{RCOOH}$ & 0.25152 & 0.17858 & -838.22256 & -837.97104 & 91.0 & -838.04397 & 86.3 \\
\hline
\end{tabular}


Table S3. The zero-point energies ( $Z P E$, hartree), thermal correction to Gibbs free energy $\left(G_{0}\right.$, hartree) of various species calculated at the B3LYP/6-31++G(d, p), SDD level in the gas phase under atmospheric pressure and room temperature (298.15 K and 1 atm). Polarizable continuum model correction energies ( $P C M-E$, hartree), total energies ( $E_{\mathrm{c}}$, hartree) corrected by $Z P E$, sum of electronic and thermal free energies $\left(G_{\mathrm{c}}\right.$, hartree) corrected by $G_{0}$, relative energies $\left(E_{\mathrm{r}}, \mathrm{kJ} \mathrm{mol}^{-1}\right)$ and relative Gibbs free energies $\left(G_{\mathrm{r}}, \mathrm{kJ}^{\mathrm{mol}}{ }^{-1}\right)$ to $\mathrm{Cd}(\mathrm{OA})_{2}+2 \mathrm{SePPh}_{2} \mathrm{H}$ for all the species in the reaction of $\mathbf{C}+\mathrm{SePPh}_{2} \mathrm{H} \rightarrow \mathbf{E}+\mathrm{RCOOH}$ and $\mathbf{C}+\mathrm{SePPh}_{2} \mathrm{H} \rightarrow \mathbf{F}+\mathrm{HPPh}_{2}$ calculated at the M06//B3LYP/6-31++G(d, p), SDD level in 1-octadecene solution under room temperature and atmospheric pressure (298.15 K and $1 \mathrm{~atm})$.

M06//B3LYP/6-31++G(d, p), SDD

Species

(298.15 $\mathrm{K}$ and $1 \mathrm{~atm})$

\begin{tabular}{|c|c|c|c|c|c|c|c|}
\hline \multirow{2}{*}{ Species } & \\
\hline & $Z P E$ & $G_{0}$ & $P C M-E$ & $\begin{array}{c}E_{\mathrm{c}}(P C M-E+ \\
Z P E)\end{array}$ & $E_{\mathrm{r}}$ & $G_{\mathrm{c}}\left(P C M-E+G_{0}\right)$ & $G_{\mathrm{r}}$ \\
\hline $\mathrm{SePPh}_{2} \mathrm{H}$ & 0.19343 & 0.15007 & -814.22030 & -814.02687 & & -814.07023 & \\
\hline $\mathrm{Cd}(\mathrm{OA})_{2}$ & 0.15896 & 0.11333 & -703.16954 & -703.01058 & & -703.05621 & \\
\hline $\mathrm{RCOOH}$ & 0.09058 & 0.06013 & -268.27275 & -268.18218 & & -268.21262 & \\
\hline $\mathrm{HPPh}_{2}$ & 0.19113 & 0.15188 & -804.87857 & -804.68745 & & -804.72669 & \\
\hline $\mathrm{Cd}(\mathrm{OA})_{2}+2 \mathrm{SePPh}_{2} \mathrm{H}$ & 0.54582 & 0.41346 & -2331.61013 & -2331.06431 & 0.0 & -2331.19667 & 0.0 \\
\hline $\mathrm{C}$ & 0.26354 & 0.20472 & -1249.14001 & -1248.87647 & & -1248.93529 & \\
\hline $\mathrm{C}+\mathrm{SePPh}_{2} \mathrm{H}+\mathrm{RCOOH}$ & 0.54754 & 0.41492 & -2331.63306 & -2331.08551 & -55.7 & -2331.21813 & -56.4 \\
\hline C/E-IM1 & 0.45782 & 0.37499 & -2063.38419 & -2062.92637 & & -2063.00920 & \\
\hline C/E-IM1 + RCOOH & 0.54840 & 0.43512 & -2331.65694 & -2331.10855 & -116.1 & -2331.22182 & -66.0 \\
\hline C/E-TS1 & 0.45450 & 0.37231 & -2063.37266 & -2062.91816 & & -2063.00035 & \\
\hline $\mathrm{C} / \mathrm{E}-\mathrm{TS} 1+\mathrm{RCOOH}$ & 0.54508 & 0.43245 & -2331.64541 & -2331.10033 & -94.6 & -2331.21297 & -42.8 \\
\hline C/E-IM2 & 0.45934 & 0.37537 & -2063.39037 & -2062.93103 & & -2063.01500 & \\
\hline $\mathrm{C} / \mathrm{E}-\mathrm{IM} 2+\mathrm{RCOOH}$ & 0.54992 & 0.43550 & -2331.66312 & -2331.11321 & -128.4 & -2331.22762 & -81.3 \\
\hline $\mathrm{E}$ & 0.36828 & 0.29778 & -1795.11131 & -1794.74302 & & -1794.81353 & \\
\hline $\mathrm{E}+2 \mathrm{RCOOH}$ & 0.54944 & 0.41804 & -2331.65681 & -2331.10737 & -113.1 & -2331.23877 & -110.5 \\
\hline $\mathrm{C}-\mathrm{i}$ & 0.26351 & 0.20406 & -1249.13693 & -1248.87342 & & -1248.93286 & \\
\hline $\mathrm{C}-\mathrm{i}+\mathrm{SePPh}_{2} \mathrm{H}+\mathrm{RCOOH}$ & 0.54751 & 0.41427 & -2331.62998 & -2331.08247 & -47.7 & -2331.21571 & -50.0 \\
\hline C-i/F-IM1 & 0.45769 & 0.37420 & -2063.38652 & -2062.92883 & & -2063.01232 & \\
\hline $\mathrm{C}-\mathrm{i} / \mathrm{F}-\mathrm{IM} 1+\mathrm{RCOOH}$ & 0.54827 & 0.43434 & -2331.65927 & -2331.11101 & -122.6 & -2331.22494 & -74.2 \\
\hline C-i/E-ii-TS1 & 0.45436 & 0.37132 & -2063.36930 & -2062.91494 & & -2062.99798 & \\
\hline $\mathrm{C}-\mathrm{i} / \mathrm{E}-\mathrm{ii}-\mathrm{TS} 1+\mathrm{RCOOH}$ & 0.54494 & 0.43145 & -2331.64205 & -2331.09711 & -86.1 & -2331.21060 & -36.6 \\
\hline C-i/E-ii-IM2 & 0.45923 & 0.37436 & -2063.38619 & -2062.92696 & & -2063.01183 & \\
\hline $\mathrm{C}-\mathrm{i} / \mathrm{E}-\mathrm{ii}-\mathrm{IM} 2+\mathrm{RCOOH}$ & 0.54980 & 0.43449 & -2331.65894 & -2331.10914 & -117.7 & -2331.22445 & -72.9 \\
\hline E-ii & 0.36805 & 0.29508 & -1795.10307 & -1794.73502 & & -1794.80799 & \\
\hline $\mathrm{E}-\mathrm{ii}+2 \mathrm{RCOOH}$ & 0.54921 & 0.41535 & -2331.64858 & -2331.09937 & -92.1 & -2331.23323 & -96.0 \\
\hline C-i/F-TS1 & 0.45718 & 0.37749 & -2063.37433 & -2062.91715 & & -2062.99684 & \\
\hline $\mathrm{C}-\mathrm{i} / \mathrm{F}-\mathrm{TS} 1+\mathrm{RCOOH}$ & 0.54776 & 0.43763 & -2331.64708 & -2331.09932 & -91.9 & -2331.20946 & -33.6 \\
\hline C-i/F-IM2 & 0.45770 & 0.37544 & -2063.39623 & -2062.93853 & & -2063.02079 & \\
\hline $\mathrm{C}-\mathrm{i} / \mathrm{F}-\mathrm{IM} 2+\mathrm{RCOOH}$ & 0.54828 & 0.43558 & -2331.66898 & -2331.12071 & -148.1 & -2331.23341 & -96.4 \\
\hline $\mathrm{F}$ & 0.26560 & 0.20565 & -1258.51366 & -1258.24806 & & -1258.30802 & \\
\hline $\mathrm{F}+\mathrm{HPPh}_{2}+\mathrm{RCOOH}$ & 0.54731 & 0.41766 & -2331.66499 & -2331.11768 & -140.1 & -2331.24733 & -133.0 \\
\hline
\end{tabular}


Table S4. The zero-point energies ( $Z P E$, hartree), thermal correction to Gibbs free energy $\left(G_{0}\right.$, hartree) of various species calculated at the B3LYP/6-31++G(d, p), SDD level in the gas phase under atmospheric pressure and room temperature (298.15 K and 1 atm). Polarizable continuum model correction energies (PCM-E, hartree), total energies ( $E_{\mathrm{c}}$, hartree) corrected by $Z P E$, sum of electronic and thermal free energies $\left(G_{\mathrm{c}}\right.$, hartree) corrected by $G_{0}$, relative energies $\left(E_{\mathrm{r}}, \mathrm{kJ} \mathrm{mol}^{-1}\right)$ and relative Gibbs free energies $\left(G_{\mathrm{r}}, \mathrm{kJ} \mathrm{mol}^{-1}\right)$ to $\mathrm{Cd}(\mathrm{OA})_{2}+\mathrm{SePPh}_{2} \mathrm{H}+\mathrm{HPPh}_{2}$ for all the species in the reaction of $\mathrm{Cd}(\mathrm{OA})_{2}+\mathrm{SePPh}_{2} \mathrm{H}+\mathrm{HPPh}_{2} \rightarrow \mathbf{G}+2 \mathrm{RCOOH}\left(\mathbf{C}+\mathrm{HPPh} \mathbf{C}_{2} \rightarrow\right.$ $\mathbf{G}+\mathrm{RCOOH}, \mathbf{E}+\mathrm{HPPh}_{2} \rightarrow \mathbf{G}+\mathrm{SePPh}_{2} \mathrm{H}$, and $\left.\mathbf{F}+2 \mathrm{HPPh}_{2} \rightarrow \mathbf{G}+\mathrm{RCOOH}+\mathrm{SePPh}_{2} \mathrm{H}\right)$ calculated at the M06//B3LYP/6-31++G(d, p), SDD level in 1-octadecene solution under room temperature and atmospheric pressure (298.15 K and $1 \mathrm{~atm})$.

M06//B3LYP/6-31++G(d, p), SDD

\begin{tabular}{|c|c|c|c|c|c|c|c|}
\hline \multirow{2}{*}{ Species } & \multicolumn{7}{|c|}{$(298.15 \mathrm{~K}$ and $1 \mathrm{~atm})$} \\
\hline & $Z P E$ & $G_{0}$ & $P C M-E$ & $\begin{array}{c}E_{\mathrm{c}}(P C M-E+ \\
Z P E)\end{array}$ & $E_{\mathrm{r}}$ & $G_{\mathrm{c}}\left(P C M-E+G_{0}\right)$ & $G_{\mathrm{r}}$ \\
\hline $\mathrm{Cd}(\mathrm{OA})_{2}$ & 0.15896 & 0.11333 & -703.16954 & -703.01058 & & -703.05621 & \\
\hline $\mathrm{SePPh}_{2} \mathrm{H}$ & 0.19343 & 0.15007 & -814.22030 & -814.02687 & & -814.07023 & \\
\hline $\mathrm{HPPh}_{2}$ & 0.19113 & 0.15188 & -804.87857 & -804.68745 & & -804.72669 & \\
\hline $\mathrm{RCOOH}$ & 0.09058 & 0.06013 & -268.27275 & -268.18218 & & -268.21262 & \\
\hline $\mathrm{Cd}(\mathrm{OA})_{2}+\mathrm{SePPh}_{2} \mathrm{H}+\mathrm{HPPh}_{2}$ & 0.54352 & 0.41528 & -2322.26841 & -2321.72489 & 0.0 & -2321.85313 & 0.0 \\
\hline $\mathrm{C}$ & 0.26354 & 0.20472 & -1249.14001 & -1248.87647 & & -1248.93529 & \\
\hline $\mathrm{C}+\mathrm{HPPh}_{2}+\mathrm{RCOOH}$ & 0.54524 & 0.41674 & -2322.29133 & -2321.74609 & -55.7 & -2321.87459 & -56.4 \\
\hline C/G-IM1 & 0.45605 & 0.37700 & -2054.02594 & -2053.56989 & & -2053.64894 & \\
\hline $\mathrm{C} / \mathrm{G}-\mathrm{IM} 1+\mathrm{RCOOH}$ & 0.54663 & 0.43713 & -2322.29869 & -2321.75207 & -71.3 & -2321.86156 & -22.1 \\
\hline $\mathrm{C} / \mathrm{G}-\mathrm{TS} 1$ & 0.45276 & 0.37436 & -2053.99356 & -2053.54080 & & -2053.61920 & \\
\hline $\mathrm{C} / \mathrm{G}-\mathrm{TS} 1+\mathrm{RCOOH}$ & 0.54334 & 0.43449 & -2322.26631 & -2321.72298 & 5.0 & -2321.83182 & 55.9 \\
\hline C/G-IM2 & 0.45813 & 0.37985 & -2054.01660 & -2053.55847 & & -2053.63675 & \\
\hline $\mathrm{C} / \mathrm{G}-\mathrm{IM} 2+\mathrm{RCOOH}$ & 0.54871 & 0.43999 & -2322.28935 & -2321.74065 & -41.4 & -2321.84937 & 9.9 \\
\hline G & 0.36683 & 0.29875 & -1785.73426 & -1785.36743 & & -1785.43551 & \\
\hline $\mathrm{G}+2 \mathrm{RCOOH}$ & 0.54798 & 0.41901 & -2322.27976 & -2321.73178 & -18.1 & -2321.86075 & -20.0 \\
\hline E & 0.36828 & 0.29778 & -1795.11131 & -1794.74302 & & -1794.81353 & \\
\hline $\mathrm{E}+\mathrm{HPPh}_{2}+2 \mathrm{RCOOH}-\mathrm{SePPh}_{2} \mathrm{H}$ & 0.54713 & 0.41986 & -2322.31508 & -2321.76795 & -113.1 & -2321.89523 & -110.5 \\
\hline E/G-IM1 & 0.56051 & 0.46812 & -2599.99251 & -2599.43200 & & -2599.52439 & \\
\hline $\mathrm{E} / \mathrm{G}-\mathrm{IM} 1+2 \mathrm{RCOOH}-\mathrm{SePPh}_{2} \mathrm{H}$ & 0.54823 & 0.43832 & -2322.31772 & -2321.76948 & -117.1 & -2321.87940 & -69.0 \\
\hline E/G-TS1 & 0.55691 & 0.46766 & -2599.95623 & -2599.39933 & & -2599.48857 & \\
\hline $\mathrm{E} / \mathrm{G}-\mathrm{TS} 1+2 \mathrm{RCOOH}-\mathrm{SePPh}_{2} \mathrm{H}$ & 0.54463 & 0.43786 & -2322.28144 & -2321.73681 & -31.3 & -2321.84358 & 25.1 \\
\hline E/G-IM2 & 0.56092 & 0.46950 & -2599.97183 & -2599.41091 & & -2599.50233 & \\
\hline $\mathrm{E} / \mathrm{G}-\mathrm{IM} 2+2 \mathrm{RCOOH}-\mathrm{SePPh}_{2} \mathrm{H}$ & 0.54864 & 0.43970 & -2322.29704 & -2321.74840 & -61.7 & -2321.85734 & -11.1 \\
\hline $\mathrm{F}$ & 0.26560 & 0.20565 & -1258.51366 & -1258.24806 & & -1258.30802 & \\
\hline $\mathrm{F}+2 \mathrm{HPPh}_{2}+\mathrm{RCOOH}-\mathrm{SePPh}_{2} \mathrm{H}$ & 0.54500 & 0.41948 & -2322.32326 & -2321.77826 & -140.1 & -2321.90379 & -133.0 \\
\hline $\mathrm{C}-\mathrm{i} / \mathrm{F}-\mathrm{IM} 2$ & 0.45770 & 0.37544 & -2063.39623 & -2062.93853 & & -2063.02079 & \\
\hline $\mathrm{C}-\mathrm{i} / \mathrm{F}-\mathrm{IM} 2+\mathrm{HPPh}_{2}+\mathrm{RCOOH}-\mathrm{SePPh}_{2} \mathrm{H}$ & 0.54598 & 0.43739 & -2322.32726 & -2321.78128 & -148.1 & -2321.88986 & -96.4 \\
\hline F/E-i-TS1a & 0.45443 & 0.37083 & -2063.37019 & -2062.91576 & & -2062.99935 & \\
\hline $\mathrm{F} / \mathrm{E}-\mathrm{i}-\mathrm{TS} 1 \mathrm{a}+\mathrm{HPPh}_{2}+\mathrm{RCOOH}-\mathrm{SePPh}_{2} \mathrm{H}$ & 0.54270 & 0.43278 & -2322.30121 & -2321.75851 & -88.3 & -2321.86843 & -40.2 \\
\hline F/E-i-IM2a & 0.45992 & 0.37795 & -2063.38554 & -2062.92562 & & -2063.00759 & \\
\hline
\end{tabular}




\begin{tabular}{|c|c|c|c|c|c|c|c|}
\hline $\mathrm{F} / \mathrm{E}-\mathrm{i}-\mathrm{IM} 2 \mathrm{a}+\mathrm{HPPh}_{2}+\mathrm{RCOOH}-\mathrm{SePPh}_{2} \mathrm{H}$ & 0.54819 & 0.43989 & -2322.31657 & -2321.76838 & -114.2 & -2321.87667 & -61.8 \\
\hline F/E-i-TS1b & 0.45428 & 0.37386 & -2063.34714 & -2062.89286 & & -2062.97329 & \\
\hline $\mathrm{F} / \mathrm{E}-\mathrm{i}-\mathrm{TS} 1 \mathrm{~b}+\mathrm{HPPh}_{2}+\mathrm{RCOOH}-\mathrm{SePPh}_{2} \mathrm{H}$ & 0.54255 & 0.43581 & -2322.27817 & -2321.73562 & -28.2 & -2321.84236 & 28.3 \\
\hline F/E-i-IM2b & 0.45992 & 0.37639 & -2063.37689 & -2062.91697 & & -2063.00050 & \\
\hline $\mathrm{F} / \mathrm{E}-\mathrm{i}-\mathrm{IM} 2 \mathrm{~b}+\mathrm{HPPh}_{2}+\mathrm{RCOOH}-\mathrm{SePPh}_{2} \mathrm{H}$ & 0.54819 & 0.43834 & -2322.30792 & -2321.75973 & -91.5 & -2321.86957 & -43.2 \\
\hline E-i & 0.36870 & 0.29776 & -1795.10594 & -1794.73724 & & -1794.80818 & \\
\hline $\mathrm{E}-\mathrm{i}+\mathrm{HPPh}_{2}+2 \mathrm{RCOOH}-\mathrm{SePPh}_{2} \mathrm{H}$ & 0.54755 & 0.41985 & -2322.30972 & -2321.76217 & -97.9 & -2321.88987 & -96.5 \\
\hline E-i/G-IM1 & 0.56055 & 0.47270 & -2599.98984 & -2599.42929 & & -2599.51714 & \\
\hline $\mathrm{E}-\mathrm{i} / \mathrm{G}-\mathrm{IM} 1+2 \mathrm{RCOOH}-\mathrm{SePPh}_{2} \mathrm{H}$ & 0.54828 & 0.44289 & -2322.31505 & -2321.76677 & -110.0 & -2321.87215 & -49.9 \\
\hline E-i/G-TS1 & 0.56018 & 0.47263 & -2599.96850 & -2599.40832 & & -2599.49587 & \\
\hline E-i/G-IM2 & 0.56070 & 0.46717 & -2599.97225 & -2599.41155 & & -2599.50508 & \\
\hline $\mathrm{E}-\mathrm{i} / \mathrm{G}-\mathrm{IM} 2+2 \mathrm{RCOOH}-\mathrm{SePPh}_{2} \mathrm{H}$ & 0.54843 & 0.43737 & -2322.29746 & -2321.74903 & -63.4 & -2321.86009 & -18.3 \\
\hline G-i & 0.36681 & 0.29785 & -1785.72993 & -1785.36312 & & -1785.43207 & \\
\hline $\mathrm{G}-\mathrm{i}+2 \mathrm{RCOOH}$ & 0.54796 & 0.41812 & -2322.27543 & -2321.72747 & -6.8 & -2321.85731 & -11.0 \\
\hline
\end{tabular}


Table S5. The zero-point energies ( $Z P E$, hartree), thermal correction to Gibbs free energy $\left(G_{0}\right.$, hartree) of various species calculated at the B3LYP/6-31++G(d, p), SDD level in the gas phase under atmospheric pressure and room temperature (298.15 K and 1 atm). Polarizable continuum model correction energies (PCM-E, hartree), total energies ( $E_{\mathrm{c}}$, hartree) corrected by $Z P E$, sum of electronic and thermal free energies $\left(G_{\mathrm{c}}\right.$, hartree) corrected by $G_{0}$, relative energies $\left(E_{\mathrm{r}}, \mathrm{kJ} \mathrm{mol}^{-1}\right)$ and relative Gibbs free energies $\left(G_{\mathrm{r}}, \mathrm{kJ} \mathrm{mol}^{-1}\right)$ to $\mathrm{Cd}(\mathrm{OA})_{2}+\mathrm{SePPh}_{2} \mathrm{H}+\mathrm{RNH}_{2}$ for all the species in the reaction of $\mathrm{Cd}(\mathrm{OA})_{2}+\mathrm{SePPh}_{2} \mathrm{H}+\mathrm{RNH}_{2} \rightarrow \mathbf{H}+2 \mathrm{RCOOH}\left((13) \mathbf{C}+\mathrm{RNH}_{2}\right.$ $\rightarrow \mathbf{H}+\mathrm{RCOOH}$, (14) $\mathbf{E}+\mathrm{RNH}_{2} \rightarrow \mathbf{H}+\mathrm{SePPh}_{2} \mathrm{H},(15) \mathbf{F}+\mathrm{RNH}_{2}+\mathrm{HPPh}_{2} \rightarrow \mathbf{H}+\mathrm{RCOOH}+\mathrm{SePPh}_{2} \mathrm{H}$, and (16) $\mathbf{G}+\mathrm{RNH}_{2} \rightarrow \mathbf{H}$ $+\mathrm{HPPh}_{2}$ ) calculated at the M06//B3LYP/6-31++G(d, p), SDD level in 1-octadecene solution under room temperature and atmospheric pressure (298.15 K and $1 \mathrm{~atm})$.

M06//B3LYP/6-31++G(d, p), SDD

Species

(298.15 K and $1 \mathrm{~atm})$

\begin{tabular}{|c|c|c|c|c|c|c|c|}
\hline \multirow{2}{*}{ Species } & & & & & & & \\
\hline & $Z P E$ & $G_{0}$ & $P C M-E$ & $\begin{array}{c}E_{\mathrm{c}}(P C M-E+ \\
Z P E)\end{array}$ & $E_{\mathrm{r}}$ & $G_{\mathrm{c}}\left(P C M-E+G_{0}\right)$ & $G_{\mathrm{r}}$ \\
\hline $\mathrm{Cd}(\mathrm{OA})_{2}$ & 0.15896 & 0.11333 & -703.16954 & -703.01058 & & -703.05621 & \\
\hline $\mathrm{SePPh}_{2} \mathrm{H}$ & 0.19343 & 0.15007 & -814.22030 & -814.02687 & & -814.07023 & \\
\hline $\mathrm{HPPh}_{2}$ & 0.19113 & 0.15188 & -804.87857 & -804.68745 & & -804.72669 & \\
\hline $\mathrm{RNH}_{2}$ & 0.09263 & 0.06713 & -135.08775 & -134.99512 & & -135.02062 & \\
\hline $\mathrm{RCOOH}$ & 0.09058 & 0.06013 & -268.27275 & -268.18218 & & -268.21262 & \\
\hline $\mathrm{Cd}(\mathrm{OA})_{2}+\mathrm{SePPh}_{2} \mathrm{H}+\mathrm{RNH}_{2}$ & 0.44502 & 0.33052 & -1652.47759 & -1652.03257 & 0.0 & -1652.14707 & 0.0 \\
\hline $\mathrm{C}$ & 0.26354 & 0.20472 & -1249.14001 & -1248.87647 & & -1248.93529 & \\
\hline $\mathrm{C}+\mathrm{RNH}_{2}+\mathrm{RCOOH}$ & 0.44675 & 0.33198 & -1652.50051 & -1652.05377 & -55.7 & -1652.16853 & -56.4 \\
\hline C/H-IM1 & 0.35888 & 0.29239 & -1384.25580 & -1383.89692 & & -1383.96341 & \\
\hline $\mathrm{C} / \mathrm{H}-\mathrm{IM} 1+\mathrm{RCOOH}$ & 0.44945 & 0.35252 & -1652.52855 & -1652.07910 & -122.2 & -1652.17603 & -76.0 \\
\hline $\mathrm{C} / \mathrm{H}-\mathrm{TS} 1$ & 0.35333 & 0.28647 & -1384.19907 & -1383.84574 & & -1383.91261 & \\
\hline $\mathrm{C} / \mathrm{H}-\mathrm{TS} 1+\mathrm{RCOOH}$ & 0.44391 & 0.34660 & -1652.47182 & -1652.02792 & 12.2 & -1652.12522 & 57.4 \\
\hline C/H-IM2 & 0.35697 & 0.28850 & -1384.20501 & -1383.84804 & & -1383.91652 & \\
\hline $\mathrm{C} / \mathrm{H}-\mathrm{IM} 2+\mathrm{RCOOH}$ & 0.44755 & 0.34863 & -1652.47776 & -1652.03021 & 6.2 & -1652.12914 & 47.1 \\
\hline $\mathrm{H}$ & 0.26554 & 0.21028 & -1115.92032 & -1115.65477 & & -1115.71003 & \\
\hline $\mathrm{H}+2 \mathrm{RCOOH}$ & 0.44670 & 0.33055 & -1652.46582 & -1652.01912 & 35.3 & -1652.13527 & 31.0 \\
\hline $\mathrm{E}$ & 0.36828 & 0.29778 & -1795.11131 & -1794.74302 & & -1794.81353 & \\
\hline $\mathrm{E}+\mathrm{RNH} 2+2 \mathrm{RCOOH}-\mathrm{SePPh}_{2} \mathrm{H}$ & 0.44864 & 0.33510 & -1652.52426 & -1652.07563 & -113.0 & -1652.18916 & -110.5 \\
\hline E/H-IM1 & 0.46326 & 0.38211 & -1930.21644 & -1929.75317 & & -1929.83433 & \\
\hline $\mathrm{E} / \mathrm{H}-\mathrm{IM} 1+2 \mathrm{RCOOH}-\mathrm{SePPh}_{2} \mathrm{H}$ & 0.45099 & 0.35231 & -1652.54164 & -1652.09066 & -152.5 & -1652.18933 & -111.0 \\
\hline E/H-TS1 & 0.45824 & 0.38343 & -1930.16117 & -1929.70293 & & -1929.77775 & \\
\hline $\mathrm{E} / \mathrm{H}-\mathrm{TS} 1+2 \mathrm{RCOOH}-\mathrm{SePPh}_{2} \mathrm{H}$ & 0.44597 & 0.35363 & -1652.48638 & -1652.04042 & -20.6 & -1652.13276 & 37.6 \\
\hline E/H-IM2 & 0.45998 & 0.38144 & -1930.16123 & -1929.70124 & & -1929.77979 & \\
\hline $\mathrm{E} / \mathrm{H}-\mathrm{IM} 2+2 \mathrm{RCOOH}-\mathrm{SePPh}_{2} \mathrm{H}$ & 0.44771 & 0.35164 & -1652.48643 & -1652.03873 & -16.2 & -1652.13480 & 32.2 \\
\hline $\mathrm{F}$ & 0.26560 & 0.20565 & -1258.51366 & -1258.24806 & & -1258.30802 & \\
\hline $\mathrm{F}+\mathrm{RNH}_{2}+\mathrm{HPPh}_{2}+\mathrm{RCOOH}-\mathrm{SePPh}_{2} \mathrm{H}$ & 0.44651 & 0.33472 & -1652.53244 & -1652.08594 & -140.1 & -1652.19772 & -133.0 \\
\hline F/I-i-IM1 & 0.36052 & 0.28819 & -1393.62385 & -1393.26333 & & -1393.33566 & \\
\hline $\mathrm{F} / \mathrm{I}-\mathrm{i}-\mathrm{IM} 1+\mathrm{HPPh}_{2}+\mathrm{RCOOH}-\mathrm{SePPh}_{2} \mathrm{H}$ & 0.44879 & 0.35014 & -1652.55487 & -1652.10608 & -193.0 & -1652.20473 & -151.4 \\
\hline F/I-i-TS1 & 0.35625 & 0.28939 & -1393.57228 & -1393.21603 & & -1393.28288 & \\
\hline
\end{tabular}




\begin{tabular}{|c|c|c|c|c|c|c|c|}
\hline $\mathrm{F} / \mathrm{I}-\mathrm{i}-\mathrm{TS} 1+\mathrm{HPPh}_{2}+\mathrm{RCOOH}-\mathrm{SePPh}_{2} \mathrm{H}$ & 0.44452 & 0.35134 & -1652.50330 & -1652.05878 & -68.8 & -1652.15196 & -12.8 \\
\hline F/I-i-IM2 & 0.35816 & 0.28859 & -1393.57368 & -1393.21552 & & -1393.28509 & \\
\hline $\mathrm{F} / \mathrm{I}-\mathrm{i}-\mathrm{IM} 2+\mathrm{HPPh}_{2}+\mathrm{RCOOH}-\mathrm{SePPh}_{2} \mathrm{H}$ & 0.44643 & 0.35054 & -1652.50470 & -1652.05827 & -67.5 & -1652.15417 & -18.6 \\
\hline $\mathrm{I}-\mathrm{i}$ & 0.26735 & 0.20908 & -1125.29034 & -1125.02298 & & -1125.08126 & \\
\hline $\mathrm{I}-\mathrm{i}+\mathrm{HPPh}_{2}+2 \mathrm{RCOOH}-\mathrm{SePPh}_{2} \mathrm{H}$ & 0.44620 & 0.33116 & -1652.49412 & -1652.04791 & -40.3 & -1652.16295 & -41.7 \\
\hline I-i/H-IM1 & 0.45955 & 0.38058 & -1930.17208 & -1929.71253 & & -1929.79150 & \\
\hline $\mathrm{I}-\mathrm{i} / \mathrm{H}-\mathrm{IM} 1+2 \mathrm{RCOOH}-\mathrm{SePPh}_{2} \mathrm{H}$ & 0.44727 & 0.35078 & -1652.49729 & -1652.05001 & -45.8 & -1652.14651 & 1.5 \\
\hline I-i/H-TS1 & 0.45907 & 0.38242 & -1930.14891 & -1929.68984 & & -1929.76649 & \\
\hline $\mathrm{I}-\mathrm{i} / \mathrm{H}-\mathrm{TS} 1+2 \mathrm{RCOOH}-\mathrm{SePPh}_{2} \mathrm{H}$ & 0.44680 & 0.35262 & -1652.47412 & -1652.02732 & 13.8 & -1652.12150 & 67.1 \\
\hline I-i/H-IM2 & 0.45924 & 0.37728 & -1930.15870 & -1929.69946 & & -1929.78142 & \\
\hline $\mathrm{I}-\mathrm{i} / \mathrm{H}-\mathrm{IM} 2+2 \mathrm{RCOOH}-\mathrm{SePPh}_{2} \mathrm{H}$ & 0.44696 & 0.34748 & -1652.48390 & -1652.03694 & -11.5 & -1652.13643 & 27.9 \\
\hline $\mathrm{H}$-ii & 0.26553 & 0.20890 & -1115.91727 & -1115.65173 & & -1115.70836 & \\
\hline $\mathrm{H}-\mathrm{ii}+2 \mathrm{RCOOH}$ & 0.44669 & 0.32917 & -1652.46277 & -1652.01608 & 43.3 & -1652.13360 & 35.4 \\
\hline G & 0.36683 & 0.29875 & -1785.73426 & -1785.36743 & & -1785.43551 & \\
\hline $\mathrm{G}+\mathrm{RNH}_{2}+2 \mathrm{RCOOH}-\mathrm{HPPh}_{2}$ & 0.44948 & 0.33426 & -1652.48894 & -1652.03946 & -18.1 & -1652.15469 & -20.0 \\
\hline G/H-IM1 & 0.46200 & 0.38856 & -1920.84108 & -1920.37907 & & -1920.45252 & \\
\hline $\mathrm{G} / \mathrm{H}-\mathrm{IM} 1+2 \mathrm{RCOOH}-\mathrm{HPPh}_{2}$ & 0.45203 & 0.35694 & -1652.50801 & -1652.05598 & -61.5 & -1652.15107 & -10.5 \\
\hline G/H-TS1 & 0.45474 & 0.37634 & -1920.76693 & -1920.31219 & & -1920.39058 & \\
\hline $\mathrm{G} / \mathrm{H}-\mathrm{TS} 1+2 \mathrm{RCOOH}-\mathrm{HPPh}_{2}$ & 0.44476 & 0.34473 & -1652.43386 & -1651.98910 & 114.1 & -1652.08913 & 152.1 \\
\hline G/H-IM2 & 0.45770 & 0.38131 & -1920.79895 & -1920.34125 & & -1920.41764 & \\
\hline $\mathrm{G} / \mathrm{H}-\mathrm{IM} 2+2 \mathrm{RCOOH}-\mathrm{HPPh}_{2}$ & 0.44772 & 0.34969 & -1652.46588 & -1652.01816 & 37.8 & -1652.11619 & 81.1 \\
\hline
\end{tabular}


Table S6. The zero-point energies ( $Z P E$, hartree), thermal correction to Gibbs free energy $\left(G_{0}\right.$, hartree) of various species calculated at the B3LYP/6-31++G(d, p), SDD level in the gas phase under atmospheric pressure and room temperature (298.15 K and 1 atm). Polarizable continuum model correction energies ( $P C M-E$, hartree), total energies ( $E_{\mathrm{c}}$, hartree) corrected by $Z P E$, sum of electronic and thermal free energies $\left(G_{\mathrm{c}}\right.$, hartree) corrected by $G_{0}$, relative energies $\left(E_{\mathrm{r}}, \mathrm{kJ} \mathrm{mol}^{-1}\right)$ and relative Gibbs free energies $\left(G_{\mathrm{r}}, \mathrm{kJ}^{\mathrm{mol}}{ }^{-1}\right)$ to $\mathrm{Cd}(\mathrm{OA})_{2}+2 \mathrm{SePPh}_{2} \mathrm{H}+\mathrm{RNH}_{2}$ for all the species in the reaction of $\mathrm{Cd}(\mathrm{OA})_{2}+2 \mathrm{SePPh}_{2} \mathrm{H}+\mathrm{RNH}_{2} \rightarrow \mathbf{I}+\mathrm{HPPh}_{2}+2 \mathrm{RCOOH}(\mathbf{H}+$ $\mathrm{SePPh}_{2} \mathrm{H} \rightarrow \mathbf{I}+\mathrm{HPPh}_{2}, \mathbf{I}-\mathbf{i} \rightarrow \mathbf{I}$, and $\mathbf{E}-\mathbf{i}+\mathrm{RNH}_{2} \rightarrow \mathbf{I}+\mathrm{HPPh}_{2}$ ) calculated at the M06//B3LYP/6-31++G(d, p), SDD level in 1 -octadecene solution under room temperature and atmospheric pressure (298.15 K and $1 \mathrm{~atm})$.

M06//B3LYP/6-31++G(d, p), SDD

Species

(298.15 $\mathrm{K}$ and $1 \mathrm{~atm})$

\begin{tabular}{|c|c|c|c|c|c|c|c|}
\hline \multirow{2}{*}{ Species } & \\
\hline & $Z P E$ & $G_{0}$ & $P C M-E$ & $\begin{array}{c}E_{\mathrm{c}}(P C M-E+ \\
Z P E)\end{array}$ & $E_{\mathrm{r}}$ & $G_{\mathrm{c}}\left(P C M-E+G_{0}\right)$ & $G_{\mathrm{r}}$ \\
\hline $\mathrm{Cd}(\mathrm{OA})_{2}$ & 0.15896 & 0.11333 & -703.16954 & -703.01058 & & -703.05621 & \\
\hline $\mathrm{SePPh}_{2} \mathrm{H}$ & 0.19343 & 0.15007 & -814.22030 & -814.02687 & & -814.07023 & \\
\hline $\mathrm{HPPh}_{2}$ & 0.19113 & 0.15188 & -804.87857 & -804.68745 & & -804.72669 & \\
\hline $\mathrm{RNH}_{2}$ & 0.09263 & 0.06713 & -135.08775 & -134.99512 & & -135.02062 & \\
\hline $\mathrm{RCOOH}$ & 0.09058 & 0.06013 & -268.27275 & -268.18218 & & -268.21262 & \\
\hline $\mathrm{Cd}(\mathrm{OA})_{2}+2 \mathrm{SePPh}_{2} \mathrm{H}+\mathrm{RNH}_{2}$ & 0.63845 & 0.48059 & -2466.69789 & -2466.05943 & 0.0 & -2466.21730 & 0.0 \\
\hline $\mathrm{H}$ & 0.26554 & 0.21028 & -1115.92032 & -1115.65477 & & -1115.71003 & \\
\hline $\mathrm{H}+\mathrm{SePPh}_{2} \mathrm{H}+2 \mathrm{RCOOH}$ & 0.64012 & 0.48062 & -2466.68612 & -2466.04599 & 35.3 & -2466.20550 & 31.0 \\
\hline H/I-IM1 & 0.45961 & 0.38025 & -1930.16299 & -1929.70338 & & -1929.78275 & \\
\hline $\mathrm{H} / \mathrm{I}-\mathrm{IM} 1+2 \mathrm{RCOOH}$ & 0.64076 & 0.50051 & -2466.70850 & -2466.06774 & -21.8 & -2466.20798 & 24.5 \\
\hline H/I-TS1 & 0.45924 & 0.37939 & -1930.13944 & -1929.68019 & & -1929.76005 & \\
\hline $\mathrm{H} / \mathrm{I}-\mathrm{TS} 1+2 \mathrm{RCOOH}$ & 0.64040 & 0.49966 & -2466.68494 & -2466.04454 & 39.1 & -2466.18528 & 84.1 \\
\hline H/I-IM2 & 0.45993 & 0.38019 & -1930.16299 & -1929.70306 & & -1929.78280 & \\
\hline $\mathrm{H} / \mathrm{I}-\mathrm{IM} 2+2 \mathrm{RCOOH}$ & 0.64108 & 0.50045 & -2466.70849 & -2466.06741 & -21.0 & -2466.20804 & 24.3 \\
\hline I & 0.26729 & 0.20743 & -1125.27927 & -1125.01197 & & -1125.07184 & \\
\hline $\mathrm{I}+\mathrm{HPPh}_{2}+2 \mathrm{RCOOH}$ & 0.63957 & 0.47958 & -2466.70334 & -2466.06377 & -11.4 & -2466.22377 & -17.0 \\
\hline $\mathrm{I}-\mathrm{i}$ & 0.26735 & 0.20908 & -1125.29034 & -1125.02298 & & -1125.08126 & \\
\hline $\mathrm{I}-\mathrm{i}+\mathrm{HPPh}_{2}+2 \mathrm{RCOOH}$ & 0.63963 & 0.48123 & -2466.71441 & -2466.07478 & -40.3 & -2466.23318 & -41.7 \\
\hline $\mathrm{I}-\mathrm{i} / \mathrm{I}-\mathrm{TS} 1$ & 0.26697 & 0.21096 & -1125.23530 & -1124.96833 & & -1125.02434 & \\
\hline $\mathrm{I}-\mathrm{i} / \mathrm{I}-\mathrm{TS} 1+\mathrm{HPPh}_{2}+2 \mathrm{RCOOH}$ & 0.63925 & 0.48311 & -2466.65937 & -2466.02013 & 103.2 & -2466.17627 & 107.7 \\
\hline I-ii & 0.26725 & 0.20530 & -1125.27590 & -1125.00865 & & -1125.07060 & \\
\hline $\mathrm{I}-\mathrm{ii}+\mathrm{HPPh}_{2}+2 \mathrm{RCOOH}$ & 0.63953 & 0.47745 & -2466.69997 & -2466.06045 & -2.7 & -2466.22253 & -13.7 \\
\hline E-i & 0.26554 & 0.21028 & -1115.92032 & -1115.65477 & & -1115.71003 & \\
\hline $\mathrm{E}-\mathrm{i}+\mathrm{RNH}_{2}+2 \mathrm{RCOOH}$ & 0.64012 & 0.48062 & -2466.68612 & -2466.04599 & 35.3 & -2466.20550 & 31.0 \\
\hline E-i/I-IM1 & 0.45961 & 0.38025 & -1930.16299 & -1929.70338 & & -1929.78275 & \\
\hline E-i/I-IM1 + 2RCOOH & 0.64076 & 0.50051 & -2466.70850 & -2466.06774 & -21.8 & -2466.20798 & 24.5 \\
\hline E-i/I-TS1 & 0.45924 & 0.37939 & -1930.13944 & -1929.68019 & & -1929.76005 & \\
\hline $\mathrm{E}-\mathrm{i} / \mathrm{I}-\mathrm{TS} 1+2 \mathrm{RCOOH}$ & 0.64040 & 0.49966 & -2466.68494 & -2466.04454 & 39.1 & -2466.18528 & 84.1 \\
\hline E-i/I-IM2 & 0.45993 & 0.38019 & -1930.16299 & -1929.70306 & & -1929.78280 & \\
\hline $\mathrm{E}-\mathrm{i} / \mathrm{I}-\mathrm{IM} 2+2 \mathrm{RCOOH}$ & 0.64108 & 0.50045 & -2466.70849 & -2466.06741 & -21.0 & -2466.20804 & 24.3 \\
\hline
\end{tabular}


Table S7. Relative Gibbs free energies $\left(G_{\mathrm{r}}, \mathrm{kJ} \mathrm{mol}^{-1}\right)$ for the coordination of $\mathrm{RNH}_{2}, \mathrm{SePPh}_{2} \mathrm{H}, \mathrm{HPPh}_{2}$, and RCOOH ligands towards key intermediates (RCOOCdSe-PPh $(\mathbf{C}), \mathrm{RCOOCdSe}_{2} \mathrm{PPh}_{2}(\mathbf{F}), \mathrm{Ph}_{2} \mathrm{PSe}-\mathrm{Cd}-\mathrm{SePPh}_{2}(\mathbf{E}), \mathrm{Ph}_{2} \mathrm{P}-\mathrm{CdSe} \mathrm{PPh}_{2}(\mathbf{E}-\mathbf{i})$, $\mathrm{Ph}_{2} \mathrm{P}-\mathrm{CdSe}-\mathrm{PPh}_{2}(\mathbf{G}), \mathrm{RHN}-\mathrm{CdSe}_{2} \mathrm{PPh}_{2}(\mathbf{I}-\mathbf{i}), \mathrm{RHN}-\mathrm{CdSe}-\mathrm{PPh}_{2}(\mathbf{H})$, and $\mathrm{Ph}_{2} \mathrm{PSe}-\mathrm{CdSe}-\mathrm{NHR}(\mathbf{I})$ ), respectively, relative to the discrete reactants calculated at the M06//B3LYP/6-31++G(d, p), SDD level in 1-octadecene solution under room temperature and atmospheric pressure $(298.15 \mathrm{~K}$ and $1 \mathrm{~atm})$.

\begin{tabular}{cccccc}
\hline \multirow{2}{*}{$\begin{array}{c}\text { Key } \\
\text { intermediates }\end{array}$} & $\mathrm{RNH}_{2}$ & $\mathrm{SePPh}_{2} \mathrm{H}$ & $\mathrm{HPPh}_{2}$ & $\mathrm{RCOOH}(\mathrm{C}=\mathrm{O})$ & $\mathrm{RCOOH}(-\mathrm{OH})$ \\
\cline { 2 - 6 } $\mathbf{C}$ & -19.7 & -10.9 & 34.2 & 14.0 & 37.4 \\
$\mathbf{F}$ & -18.4 & -0.6 & 36.6 & 13.0 & 31.6 \\
$\mathbf{E}$ & -0.5 & -8.1 & 41.6 & 29.3 & 46.3 \\
$\mathbf{E}-\mathbf{i}$ & -7.4 & -8.4 & 46.5 & 34.6 & 53.3 \\
$\mathbf{G}$ & 1.5 & 8.9 & 33.8 & 29.9 & 38.0 \\
$\mathbf{I}-\mathbf{i}$ & 1.1 & -8.5 & 43.2 & 31.3 & 23.1 \\
$\mathbf{H}$ & 3.2 & -6.5 & 50.1 & 34.5 & 16.1 \\
$\mathbf{I}$ & -3.0 & -9.3 & 30.4 & 27.2 & 8.3 \\
\hline
\end{tabular}




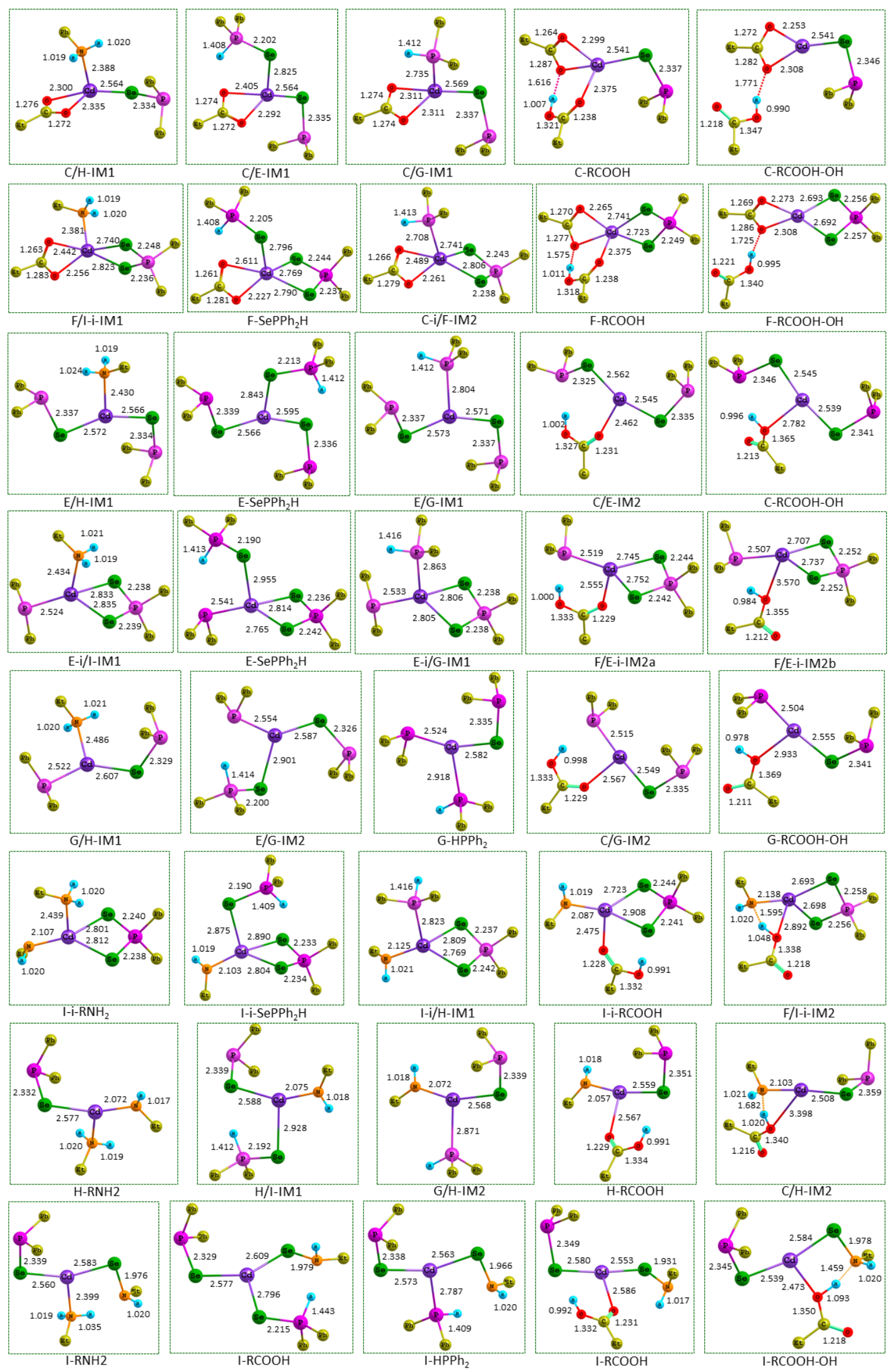

Figure S7. The geometric structures for the coordination of $\mathrm{RNH}_{2}, \mathrm{SePPh}_{2} \mathrm{H}, \mathrm{HPPh}_{2}$, and $\mathrm{RCOOH}$ toward important intermediates (RCOOCdSe- $\mathrm{PPh}_{2}$ (C), $\mathrm{RCOOCdSe}{ }_{2} \mathrm{PPh}_{2}$ (F), $\mathrm{Ph}_{2} \mathrm{PSe}-\mathrm{Cd}-\mathrm{SePPh}_{2}(\mathbf{E}), \mathrm{Ph}_{2} \mathrm{P}-\mathrm{CdSe}_{2} \mathrm{PPh}_{2}(\mathbf{E}-\mathbf{i}), \mathrm{Ph}_{2} \mathrm{P}-\mathrm{CdSe} \mathrm{PPh}_{2}$ (G), RHN-CdSe ${ }_{2} \mathrm{PPh}_{2}(\mathbf{I}-\mathbf{i}), \mathrm{RHN}-\mathrm{CdSe}-\mathrm{PPh}_{2}(\mathbf{H})$, and $\mathrm{Ph}_{2} \mathrm{PSe}-\mathrm{CdSe}-\mathrm{NHR}(\mathbf{I})$ ) calculated at the M06//B3LYP/6-31++G(d, p), SDD level in 1-octadecene solution under room temperature and atmospheric pressure (298.15 K and $1 \mathrm{~atm})$. 
Table S8. Snapshot and standard orientation of all the species in the gas phase calculated at the B3LYP/6-31++G(d, p), SDD level. Distance lengths are reported in $\AA$.

$\mathrm{SePPh}_{2} \mathrm{H}$

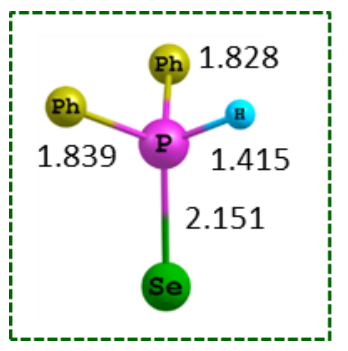

$\begin{array}{rrrr}\mathrm{P} & -0.02183300 & 0.60467600 & -0.65425600 \\ \mathrm{C} & 1.50151600 & -0.28608800 & -0.17583800 \\ \mathrm{C} & 1.78581200 & -0.51600500 & 1.17918800 \\ \mathrm{C} & 2.40666100 & -0.71455800 & -1.15698700 \\ \mathrm{C} & 2.95472000 & -1.18383200 & 1.54369100 \\ \mathrm{H} & 1.09923400 & -0.16563800 & 1.94479600 \\ \mathrm{C} & 3.58040200 & -1.37905400 & -0.78760800 \\ \mathrm{H} & 2.20401200 & -0.52738500 & -2.20847200 \\ \mathrm{C} & 3.85355700 & -1.61606500 & 0.56142000 \\ \mathrm{H} & 3.16853200 & -1.36141600 & 2.59362900 \\ \mathrm{H} & 4.27867500 & -1.70601000 & -1.55263500 \\ \mathrm{H} & 4.76565600 & -2.13112500 & 0.84884600 \\ \mathrm{C} & -1.41616200 & -0.52925700 & -0.26345200 \\ \mathrm{C} & -2.55877000 & -0.04286600 & 0.38126500 \\ \mathrm{C} & -1.34820500 & -1.88125200 & -0.63823900 \\ \mathrm{C} & -3.62767000 & -0.90271100 & 0.65198600 \\ \mathrm{H} & -2.60174100 & 1.00428800 & 0.66793900 \\ \mathrm{C} & -2.41923200 & -2.73525800 & -0.37007400 \\ \mathrm{H} & -0.46060000 & -2.27230000 & -1.12865300 \\ \mathrm{C} & -3.56040800 & -2.24616100 & 0.27548700 \\ \mathrm{H} & -4.51126200 & -0.51976300 & 1.15434900 \\ \mathrm{H} & -2.36217900 & -3.78007800 & -0.66164100 \\ \mathrm{H} & -4.39242500 & -2.91253900 & 0.48485900 \\ \mathrm{Se} & -0.23070000 & 2.61737700 & 0.07436500 \\ \mathrm{H} & 0.07007000 & 0.50963600 & -2.06261100\end{array}$

$\mathrm{HPPh}_{2}$

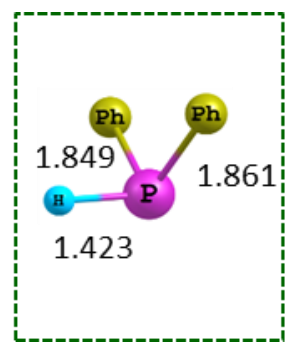

$\begin{array}{lrrr}\mathrm{P} & 0.04767600 & 1.66775400 & -0.26849400 \\ \mathrm{H} & -0.01274300 & 1.74307100 & -1.68842000\end{array}$

$\begin{array}{lrrr}\mathrm{C} & -1.43536900 & 0.58308500 & -0.06540600 \\ \mathrm{C} & -2.23731700 & 0.18364300 & -1.14730200 \\ \mathrm{C} & -1.81986300 & 0.19686100 & 1.23097400 \\ \mathrm{C} & -3.38751500 & -0.58460300 & -0.94044400 \\ \mathrm{H} & -1.96439400 & 0.47250000 & -2.15872400 \\ \mathrm{C} & -2.95870800 & -0.58307700 & 1.43720700 \\ \mathrm{H} & -1.22472100 & 0.50911900 & 2.08569500 \\ \mathrm{C} & -3.74844500 & -0.97429400 & 0.35115500 \\ \mathrm{H} & -3.99583400 & -0.88230400 & -1.79007800 \\ \mathrm{H} & -3.23539400 & -0.87715500 & 2.44577300 \\ \mathrm{H} & -4.63938800 & -1.57439300 & 0.51184100 \\ \mathrm{C} & 1.46097700 & 0.46359900 & -0.13878500 \\ \mathrm{C} & 2.65267400 & 0.92736900 & 0.43944900 \\ \mathrm{C} & 1.40353300 & -0.86508500 & -0.59079900 \\ \mathrm{C} & 3.76770500 & 0.08996200 & 0.55143600 \\ \mathrm{H} & 2.70804700 & 1.94913500 & 0.80634900 \\ \mathrm{C} & 2.51347000 & -1.70383900 & -0.47436800 \\ \mathrm{H} & 0.48614100 & -1.24857500 & -1.02801900 \\ \mathrm{C} & 3.69966000 & -1.22737700 & 0.09460400 \\ \mathrm{H} & 4.68278400 & 0.46606500 & 1.00029600 \\ \mathrm{H} & 2.45348100 & -2.72946700 & -0.82813900 \\ \mathrm{H} & 4.56206600 & -1.88176700 & 0.18451700\end{array}$

$\mathrm{SePR}_{3}$

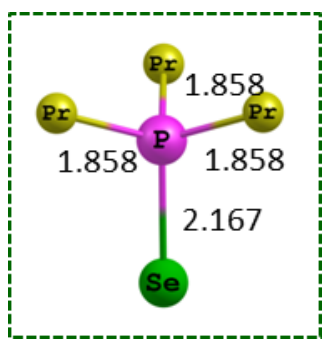

$\begin{array}{lrrr}\mathrm{P} & -0.47322800 & 0.00031000 & 0.00129200 \\ \mathrm{Se} & -2.63993800 & -0.00271600 & 0.00281300 \\ \mathrm{C} & 0.18877700 & -0.73179800 & 1.57562300 \\ \mathrm{C} & 0.18679400 & -0.99575100 & -1.42181700 \\ \mathrm{C} & 0.18251100 & 1.73204800 & -0.15502000 \\ \mathrm{H} & -0.53193400 & -1.81697900 & -1.52152700 \\ \mathrm{H} & 0.04731400 & -0.36733700 & -2.31070900 \\ \mathrm{H} & -0.53207600 & 2.22247900 & -0.82561100 \\ \mathrm{H} & 0.03408200 & 2.19431300 & 0.82919900 \\ \mathrm{H} & 0.05135600 & -1.81619700 & 1.47677100 \\ \mathrm{H} & -0.52926300 & -0.40811500 & 2.33734500 \\ \mathrm{C} & 1.61719500 & -1.56009400 & -1.36774000 \\ \mathrm{C} & 1.61974800 & -0.39995000 & 2.03227700\end{array}$




$\begin{array}{lrrr}\mathrm{C} & 1.61658200 & 1.96508300 & -0.66058500 \\ \mathrm{H} & 1.72987600 & 1.52751000 & -1.65953900 \\ \mathrm{H} & 2.34306100 & 1.46178100 & -0.01387800 \\ \mathrm{H} & 2.34958600 & -0.75556700 & -1.24251800 \\ \mathrm{H} & 1.72100000 & -2.21686000 & -0.49581500 \\ \mathrm{H} & 2.34809400 & -0.68850000 & 1.26702800 \\ \mathrm{H} & 1.72024900 & 0.68347800 & 2.16774500 \\ \mathrm{C} & 1.96647700 & -1.10381400 & 3.35212900 \\ \mathrm{H} & 1.91316300 & -2.19341200 & 3.24609300 \\ \mathrm{H} & 2.97998600 & -0.84832500 & 3.67963400 \\ \mathrm{H} & 1.27298700 & -0.81343000 & 4.14946400 \\ \mathrm{C} & 1.95476800 & -2.35694100 & -2.63627800 \\ \mathrm{H} & 1.25786000 & -3.19090800 & -2.77667500 \\ \mathrm{H} & 1.89898800 & -1.72350500 & -3.52902500 \\ \mathrm{H} & 2.96730200 & -2.77130300 & -2.58256600 \\ \mathrm{C} & 1.95120500 & 3.46222400 & -0.72983500 \\ \mathrm{H} & 1.25782300 & 3.99150400 & -1.39313200 \\ \mathrm{H} & 1.88626400 & 3.92943600 & 0.25952900 \\ \mathrm{H} & 2.96624800 & 3.62156700 & -1.10936900\end{array}$

$\mathrm{PR}_{3}$

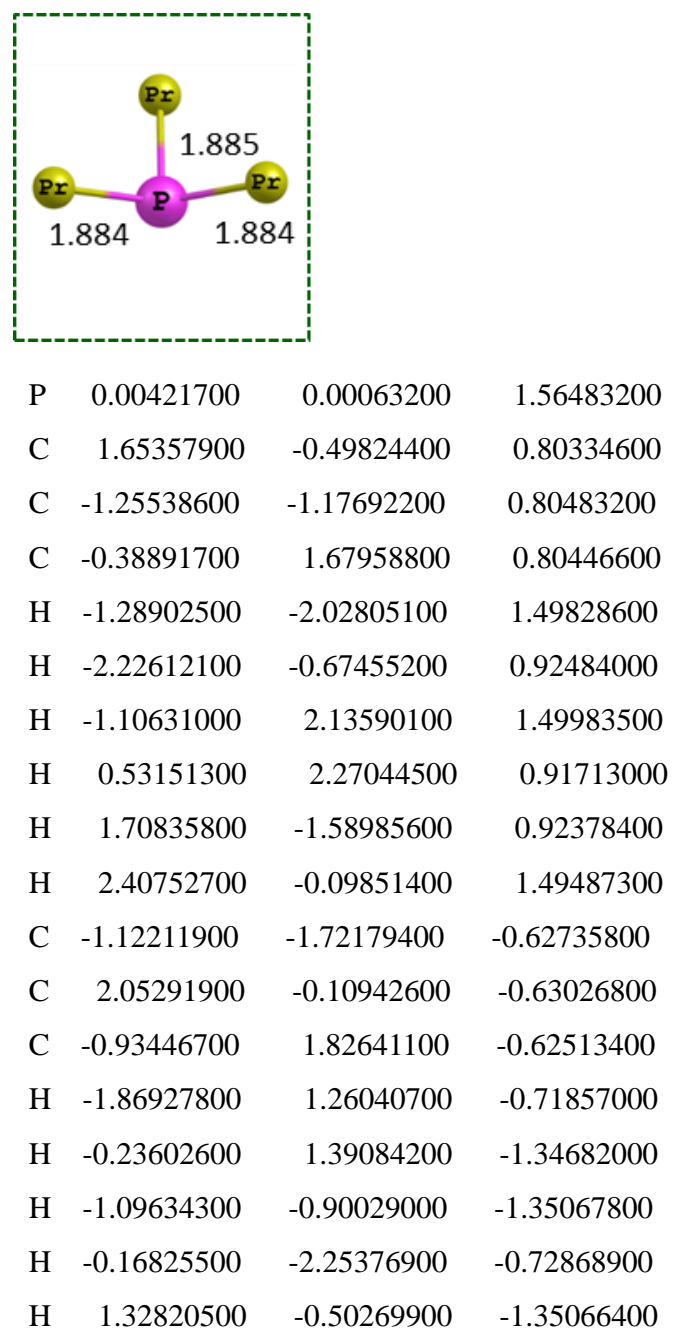

$\begin{array}{rrrr}\mathrm{H} & 2.02929700 & 0.98244100 & -0.73204600 \\ \mathrm{C} & 3.45580600 & -0.61431200 & -0.99781300 \\ \mathrm{H} & 3.50909600 & -1.70821600 & -0.94526900 \\ \mathrm{H} & 3.73279400 & -0.31537700 & -2.01506800 \\ \mathrm{H} & 4.21336400 & -0.21341000 & -0.31400600 \\ \mathrm{C} & -2.26706300 & -2.67894500 & -0.98975000 \\ \mathrm{H} & -2.30248700 & -3.53261800 & -0.30260600 \\ \mathrm{H} & -3.23811000 & -2.17235600 & -0.93736100 \\ \mathrm{H} & -2.15086900 & -3.07284100 & -2.00574200 \\ \mathrm{C} & -1.20026200 & 3.29289000 & -0.99513100 \\ \mathrm{H} & -1.91960500 & 3.75065100 & -0.30578400 \\ \mathrm{H} & -0.27876400 & 3.88557000 & -0.95214100 \\ \mathrm{H} & -1.60676200 & 3.38132600 & -2.00892000\end{array}$

TS1-1

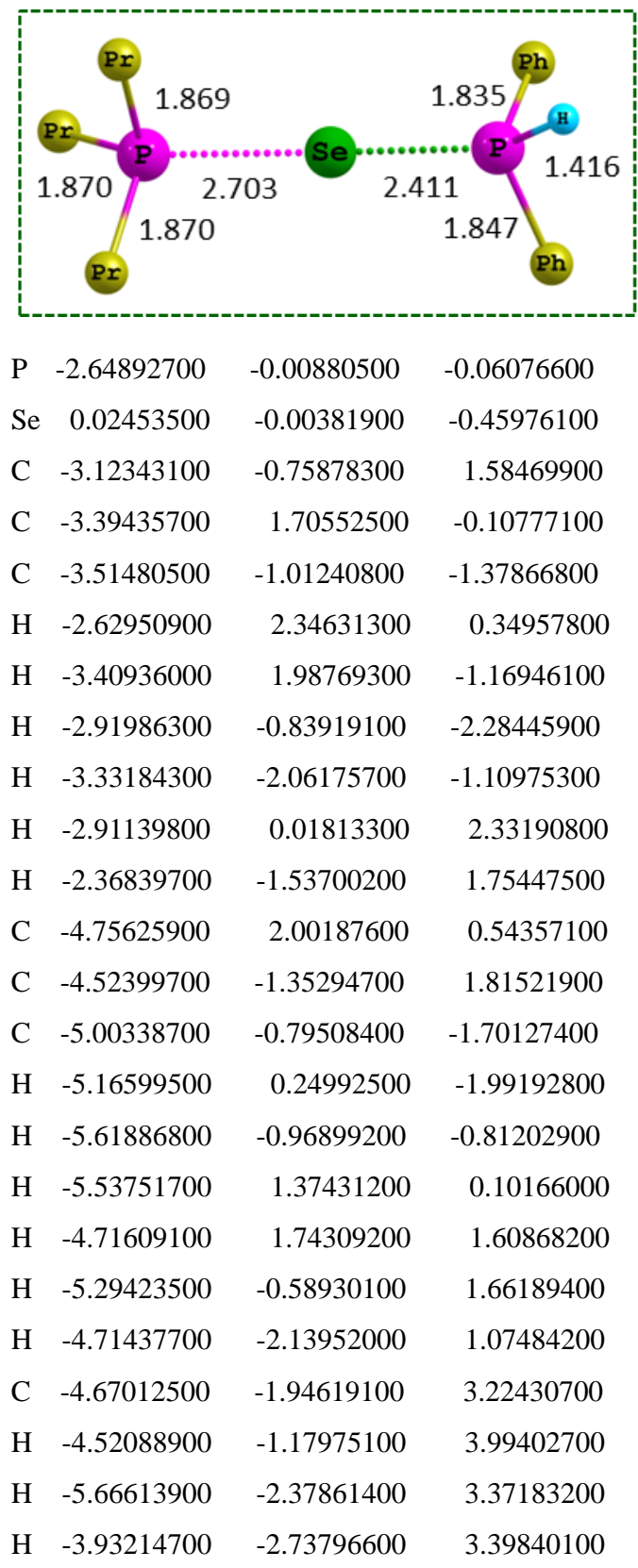




$\begin{array}{lrrr}\mathrm{C} & -5.15010100 & 3.47947700 & 0.40177700 \\ \mathrm{H} & -4.40103100 & 4.13431400 & 0.86226700 \\ \mathrm{H} & -5.23688200 & 3.76658100 & -0.65282800 \\ \mathrm{H} & -6.11317700 & 3.68189100 & 0.88369900 \\ \mathrm{C} & -5.48294700 & -1.71075600 & -2.83720900 \\ \mathrm{H} & -4.90621200 & -1.53968300 & -3.75370100 \\ \mathrm{H} & -5.37022800 & -2.76761400 & -2.56793400 \\ \mathrm{H} & -6.53958900 & -1.53584300 & -3.06906900 \\ \mathrm{P} & 2.40170800 & -0.01444500 & -0.85977800 \\ \mathrm{H} & 2.78506600 & -0.08732200 & -2.22144200 \\ \mathrm{C} & 3.37376400 & -1.43013200 & -0.21444900 \\ \mathrm{C} & 3.95184400 & -2.37075800 & -1.08105200 \\ \mathrm{C} & 3.47804100 & -1.62527500 & 1.17309600 \\ \mathrm{C} & 4.63267700 & -3.48020300 & -0.57004600 \\ \mathrm{H} & 3.87254900 & -2.24032100 & -2.15724600 \\ \mathrm{C} & 4.16660900 & -2.72727000 & 1.68099700 \\ \mathrm{H} & 3.01708700 & -0.91576600 & 1.85507300 \\ \mathrm{C} & 4.74440300 & -3.65890500 & 0.81093000 \\ \mathrm{H} & 5.07628100 & -4.20074000 & -1.25164600 \\ \mathrm{H} & 4.24644900 & -2.86352100 & 2.75590600 \\ \mathrm{H} & 5.27576400 & -4.51908500 & 1.20819100 \\ \mathrm{C} & 3.33124900 & 1.48311700 & -0.30845500 \\ \mathrm{C} & 2.62699900 & 2.53218600 & 0.29682100 \\ \mathrm{C} & 4.71930200 & 1.60083500 & -0.49328200 \\ \mathrm{C} & 3.30130200 & 3.68515100 & 0.71336600 \\ \mathrm{H} & 1.55311700 & 2.43734400 & 0.43567900 \\ \mathrm{C} & 5.38939200 & 2.75360700 & -0.08012000 \\ \mathrm{H} & 5.27899300 & 0.79022600 & -0.95232500 \\ \mathrm{C} & 4.68042400 & 3.79806000 & 0.52488000 \\ \mathrm{H} & 2.74686800 & 4.49302800 & 1.18289900 \\ \mathrm{H} & 6.46258800 & 2.83674100 & -0.22834800 \\ \mathrm{H} & 5.20348100 & 4.69423600 & 0.84716900\end{array}$

$\mathrm{RNH}_{2}$

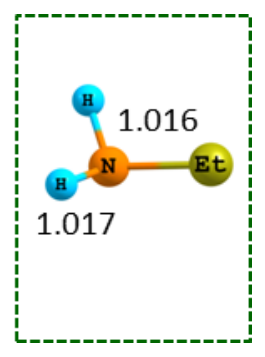
C $\quad 1.24988900$
$-0.23947700$
$-0.02923900$
H 1.31064000
$-0.77205600$
$-0.98348300$
H 1.30747100
$-0.98229000$
H 2.12135600
0.41771200
0.77615700
C -0.04775300
0.56173000

$\begin{array}{cc}\mathrm{H} & -0.06279100 \\ \mathrm{H} & -0.07431000 \\ \mathrm{~N} & -1.21041600 \\ \mathrm{H} & -1.26382800 \\ \mathrm{H} & -2.07843800\end{array}$

1.12854900

1.00344400

$\begin{array}{lll}\mathrm{H} & -0.07431000 & 1.30043100\end{array}$

$-0.75376000$$$
-0.31956000
$$

$-0.11451800$

$-0.99793800$

0.64179300

0.20899100

$-0.10711300$

\section{$\mathrm{RCOOH}$}

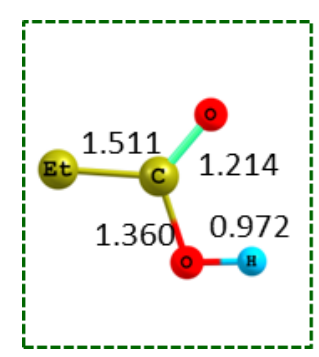

$$
\begin{array}{crrc}
\text { C } & 0.61869200 & -0.13356000 & 0.07306000 \\
\text { O } & 1.43908300 & -0.94444900 & -0.30394900 \\
\text { O } & 0.88489100 & 1.20023800 & 0.10120200 \\
\text { H } & 1.79301100 & 1.31592600 & -0.22618900 \\
\text { C }-0.78170100 & -0.44345500 & 0.54906900 \\
\text { C }-1.86819400 & 0.13721400 & -0.37412700 \\
\text { H }-0.86333300 & -1.53055700 & 0.61357800 \\
\text { H }-0.89620300 & -0.02920300 & 1.55770500 \\
\text { H }-1.77704700 & -0.26234400 & -1.38919700 \\
\text { H }-2.85996100 & -0.12840200 & 0.00384100 \\
\text { H }-1.80103400 & 1.22707200 & -0.42577500
\end{array}
$$

$\mathrm{Cd}(\mathrm{OA})_{2}$

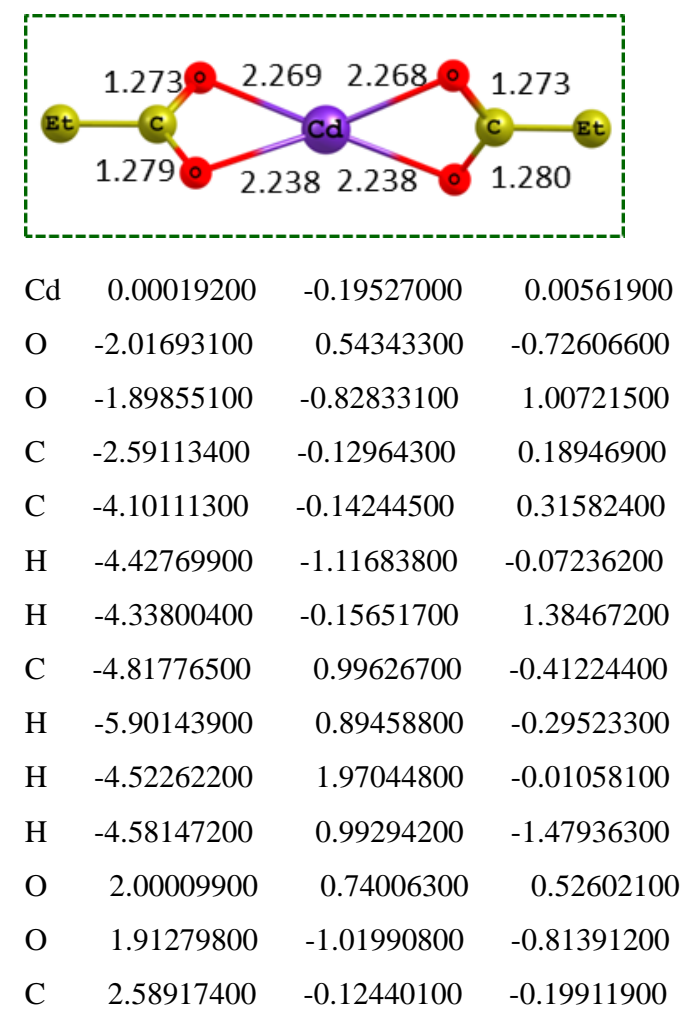




$\begin{array}{lrrr}\mathrm{C} & 4.09618100 & -0.11452200 & -0.35804600 \\ \mathrm{H} & 4.29421700 & -0.01905700 & -1.43292300 \\ \mathrm{H} & 4.44727400 & -1.11759900 & -0.08737300 \\ \mathrm{C} & 4.82515100 & 0.96746200 & 0.43942200 \\ \mathrm{H} & 4.64867800 & 0.85535700 & 1.51287300 \\ \mathrm{H} & 5.90332200 & 0.90456900 & 0.26008100 \\ \mathrm{H} & 4.48622100 & 1.96669100 & 0.15259500\end{array}$

$\left(\mathrm{SePPh}_{2} \mathrm{H}\right) \mathrm{Cd}(\mathrm{OA})_{2}$

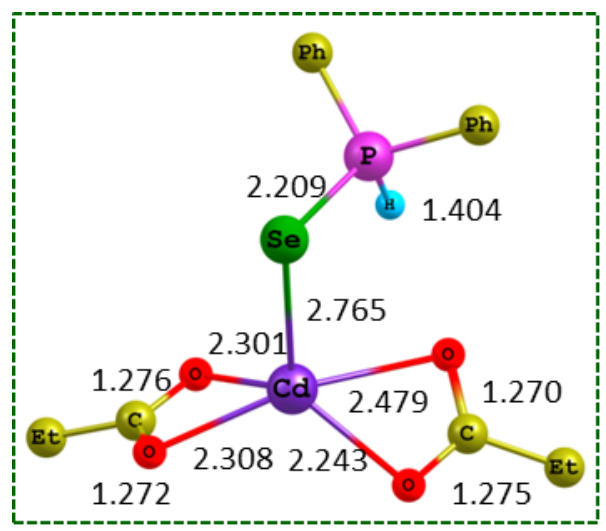

$\begin{array}{lrrr}\mathrm{Cd} & 1.95606200 & 0.31923700 & 0.17437000 \\ \mathrm{O} & 0.58799400 & 1.91916100 & -1.13562200 \\ \mathrm{O} & 2.37110200 & 2.51999300 & 0.04859400 \\ \mathrm{C} & 1.40252600 & 2.80488500 & -0.73080600 \\ \mathrm{C} & 1.20300300 & 4.24875000 & -1.17166800 \\ \mathrm{H} & 0.94778100 & 4.22924700 & -2.23714900 \\ \mathrm{H} & 0.30155000 & 4.60662800 & -0.65500500 \\ \mathrm{C} & 2.38271800 & 5.18257300 & -0.89537300 \\ \mathrm{H} & 2.14763100 & 6.20104300 & -1.22330900 \\ \mathrm{H} & 2.62361100 & 5.20668600 & 0.17051500 \\ \mathrm{H} & 3.27996300 & 4.85147900 & -1.42689600 \\ \mathrm{O} & 3.74476000 & -1.03263200 & 0.72369100 \\ \mathrm{O} & 2.74689200 & -1.31386800 & -1.23982000 \\ \mathrm{C} & 3.64387500 & -1.63511700 & -0.39177500 \\ \mathrm{C} & 4.59669000 & -2.76765500 & -0.74180400 \\ \mathrm{H} & 5.02792000 & -2.52768700 & -1.72113300 \\ \mathrm{H} & 3.97664400 & -3.65790900 & -0.90764300 \\ \mathrm{C} & 5.68772300 & -3.04183900 & 0.29392900 \\ \mathrm{H} & 5.25460000 & -3.29681000 & 1.26525700 \\ \mathrm{H} & 6.32192900 & -3.87384900 & -0.03155500 \\ \mathrm{H} & 6.32233600 & -2.16306300 & 0.44078300 \\ \mathrm{P} & -1.61274500 & -0.33738900 & -0.04055500 \\ \mathrm{C} & -2.46693200 & -1.89113800 & -0.48347000 \\ \mathrm{C} & -2.12695400 & -3.10264600 & 0.13079900 \\ \mathrm{C} & -3.45155900 & -1.86478900 & -1.48687300 \\ \mathrm{H} & -2.77119300 & -4.28227500 & -0.25345700 \\ & -1.36200300 & -3.11985100 & 0.90091200\end{array}$

$\begin{array}{cccc}\mathrm{C} & -4.08564900 & -3.04679300 & -1.86951900 \\ \mathrm{H} & -3.72647200 & -0.92902900 & -1.96556600 \\ \mathrm{C} & -3.74697200 & -4.25610400 & -1.25199500 \\ \mathrm{H} & -2.50516900 & -5.21906600 & 0.22688200 \\ \mathrm{H} & -4.84332800 & -3.02342300 & -2.64709900 \\ \mathrm{H} & -4.24353400 & -5.17466500 & -1.55094200 \\ \mathrm{C} & -2.87949200 & 0.94701500 & 0.17150600 \\ \mathrm{C} & -2.67511200 & 2.20343600 & -0.42097200 \\ \mathrm{C} & -4.02319200 & 0.70720500 & 0.94946800 \\ \mathrm{C} & -3.62888400 & 3.20948100 & -0.24444500 \\ \mathrm{H} & -1.77142100 & 2.38981000 & -0.99606000 \\ \mathrm{C} & -4.96681100 & 1.71997700 & 1.12216900 \\ \mathrm{H} & -4.18074800 & -0.26281900 & 1.41239900 \\ \mathrm{C} & -4.77107500 & 2.96998900 & 0.52423500 \\ \mathrm{H} & -3.47613800 & 4.18041700 & -0.70635100 \\ \mathrm{H} & -5.85284600 & 1.53437400 & 1.72194600 \\ \mathrm{H} & -5.50843700 & 3.75592300 & 0.65995800 \\ \mathrm{Se} & -0.20732900 & -0.57131600 & 1.64779700 \\ \mathrm{H} & -0.92674500 & 0.08381400 & -1.19091500\end{array}$

$\left(\mathrm{HPPh}_{2}\right) \mathrm{Cd}(\mathrm{OA})_{2}$

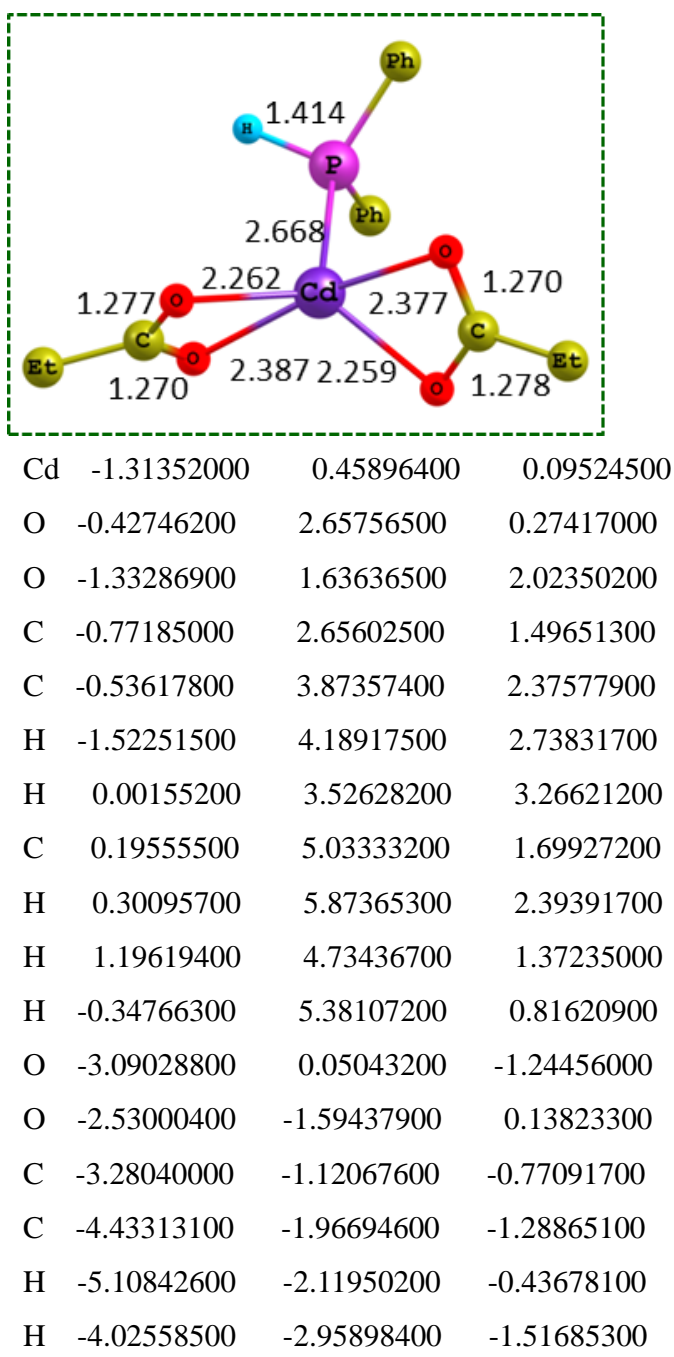




$\begin{array}{lrrr}\mathrm{C} & -5.18695800 & -1.38343400 & -2.48454000 \\ \mathrm{H} & -4.52541100 & -1.25660700 & -3.34683200 \\ \mathrm{H} & -6.00798800 & -2.04682300 & -2.77752800 \\ \mathrm{H} & -5.60390500 & -0.40130300 & -2.24599800 \\ \mathrm{P} & 0.86906600 & -0.86947200 & -0.67379100 \\ \mathrm{H} & 0.57888500 & -1.79677400 & -1.70050100 \\ \mathrm{C} & 1.48106700 & -1.98750900 & 0.65425200 \\ \mathrm{C} & 0.54733700 & -2.84998800 & 1.25646200 \\ \mathrm{C} & 2.80625500 & -1.96902100 & 1.11043400 \\ \mathrm{C} & 0.95099700 & -3.70000800 & 2.28767300 \\ \mathrm{H} & -0.49103300 & -2.84926300 & 0.93214800 \\ \mathrm{C} & 3.19897500 & -2.81686800 & 2.15107500 \\ \mathrm{H} & 3.53200300 & -1.29873600 & 0.66085400 \\ \mathrm{C} & 2.27558900 & -3.68551500 & 2.73714200 \\ \mathrm{H} & 0.22558100 & -4.36554300 & 2.74643900 \\ \mathrm{H} & 4.22731400 & -2.79554800 & 2.50053800 \\ \mathrm{H} & 2.58346100 & -4.34325600 & 3.54486000 \\ \mathrm{C} & 2.30128700 & 0.01903900 & -1.38218000 \\ \mathrm{C} & 2.41964500 & 1.40262800 & -1.16949100 \\ \mathrm{C} & 3.26588700 & -0.64995000 & -2.15756600 \\ \mathrm{C} & 3.50272200 & 2.10078700 & -1.71396800 \\ \mathrm{H} & 1.66306300 & 1.93808700 & -0.60106900 \\ \mathrm{C} & 4.34113100 & 0.05416900 & -2.70083500 \\ \mathrm{H} & 3.17777400 & -1.71826800 & -2.33647800 \\ \mathrm{C} & 4.46184400 & 1.43036900 & -2.47613100 \\ \mathrm{H} & 3.58624600 & 3.17088800 & -1.54899900 \\ \mathrm{H} & 5.08062800 & -0.46855600 & -3.30060800 \\ \mathrm{H} & 5.29816700 & 1.97753700 & -2.90180300\end{array}$

$\left(\mathrm{SePR}_{3}\right) \mathrm{Cd}(\mathrm{OA})_{2}$

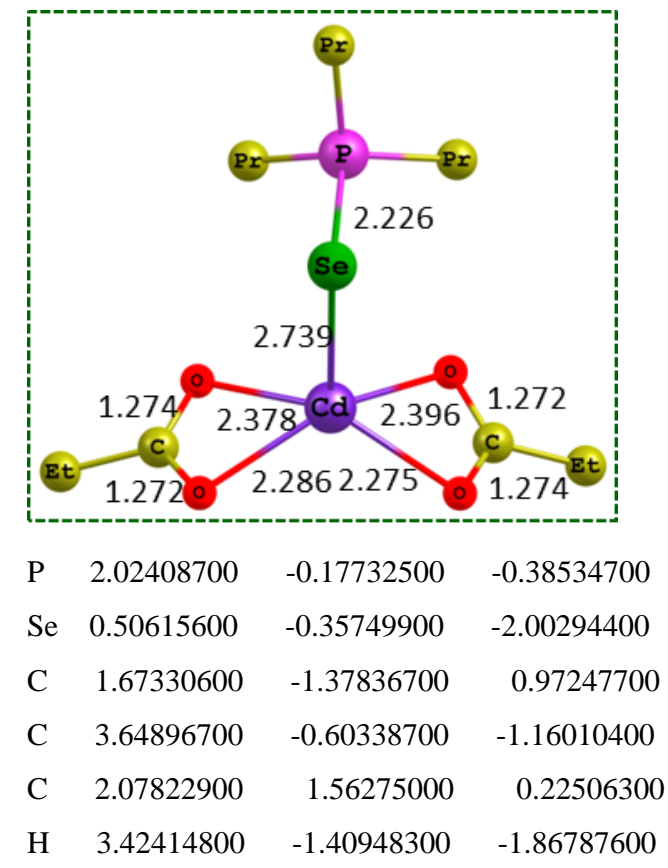

\begin{tabular}{|c|c|c|c|}
\hline & & & \\
\hline & 1.96323900 & 0 & -0.68650900 \\
\hline & 1.15281800 & 1493000 & .79500600 \\
\hline & 2.05142900 & -2.34243600 & 0.6076730 \\
\hline & 0.58300300 & 47940000 & 016476 \\
\hline & 4.79863100 & -1.03242400 & $x^{2}+2$ \\
\hline & 2.21388300 & -1.06994000 & 2.3 \\
\hline & 3.31366500 & 2.04036300 & 1.01009900 \\
\hline & 4.216 & 0 & \\
\hline & 3.463150 & 1.430 & \\
\hline & 5.02299900 & -0.24310200 & 49 \\
\hline & 4.49476500 & -1.91 & 34 \\
\hline & 3.30509600 & -0.967 & 2.371 \\
\hline & 1.80 & -6 & 2.72486600 \\
\hline & 1.81002900 & -2.17232700 & 3.37 \\
\hline & 2.23387700 & -3.14092000 & 0804000 \\
\hline & 2.16 & -1.93 & \\
\hline & 0.72129400 & -2.27927500 & 4092650 \\
\hline & 6.06721400 & -1.36712500 & -1.02662300 \\
\hline & 5.88499900 & -2.17851800 & -1.7 \\
\hline & 6.42245700 & -0.498 & 3100 \\
\hline $\mathrm{H}$ & 6.87439100 & -1.68394800 & -0.358 \\
\hline & 3.16308300 & 3.51290100 & 1.41954900 \\
\hline$\Pi$ & 3.04323500 & 4.15 & 7000 \\
\hline $\mathrm{H}$ & 2.28646400 & 9200 & 2.06014000 \\
\hline & 4.04534300 & 3.85653000 & 1.965 \\
\hline & -1.67914700 & 0.06618400 & -0.40689200 \\
\hline $\mathrm{O}$ & -1.08289700 & 1.93514500 & 967883 \\
\hline $\mathrm{O}$ & -2.71607100 & 2.08790600 & -0.528 \\
\hline $\mathrm{C}$ & -1.99725100 & 2.60278600 & 0.38754100 \\
\hline $\mathrm{C}$ & -2.22231400 & 4.04991600 & 0.80 \\
\hline $\mathrm{H}$ & 80 & & \\
\hline C & -1.29226800 & 4.58824100 & 57 \\
\hline C & -3.42132000 & 4.73703000 & 400 \\
\hline $\mathrm{H}$ & -3.50414800 & 5.77256800 & 494 \\
\hline $\mathrm{H}$ & -3.325419 & 4.7445 & .94 \\
\hline $\mathrm{H}$ & -4.35356400 & 4.21827000 & $38834 \mathrm{Y}$ \\
\hline $\mathrm{O}$ & -3.12175000 & .70565300 & -0.47963200 \\
\hline $\mathrm{O}$ & -1.62556300 & .72008900 & 16134500 \\
\hline C & -2.60189600 & -2.25570300 & 0.5433140 \\
\hline $\mathrm{C}$ & -3.15347100 & -3.57724000 & 1.05 \\
\hline $\mathrm{H}$ & -3.48581500 & -3.39666600 & 2.08810500 \\
\hline $\mathrm{H}$ & -2.30347100 & -4.26552400 & .14272900 \\
\hline C & -4.27702600 & -4.18598600 & 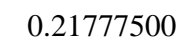 \\
\hline $\mathrm{H}$ & -3.94151400 & -4.38854100 & -0.80352 \\
\hline & 4.61735600 & -5.12805000 & 0.661740 \\
\hline
\end{tabular}


$\left(\mathrm{PR}_{3}\right) \mathrm{Cd}(\mathrm{OA})_{2}$

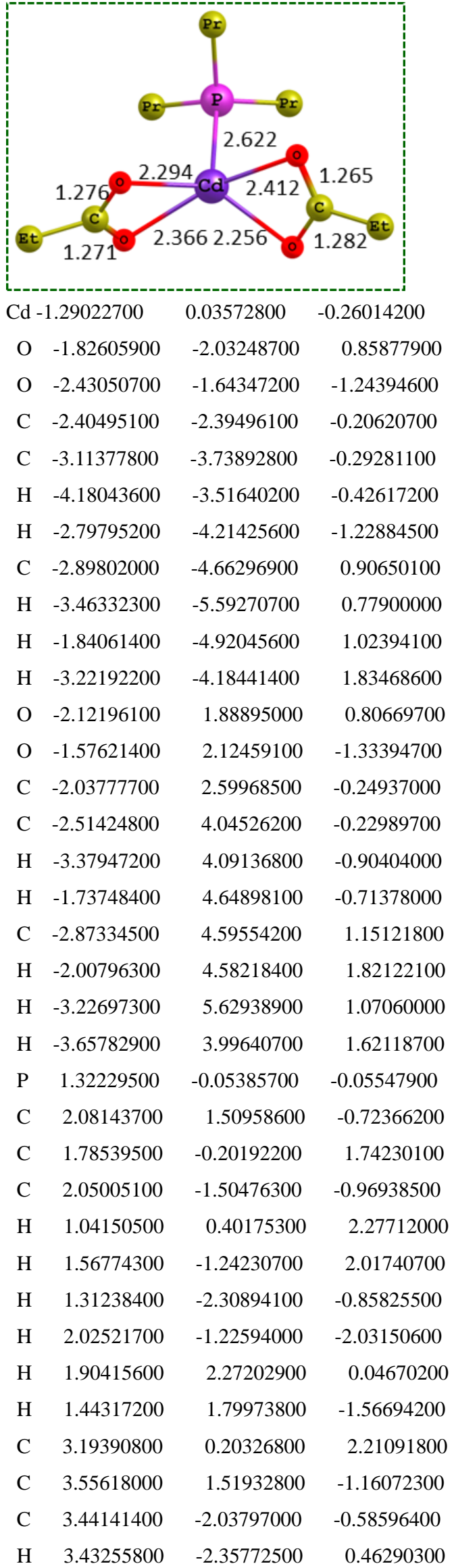

$\begin{array}{rrrr}\mathrm{H} & 4.19102700 & -1.24373000 & -0.66467100 \\ \mathrm{H} & 3.95714900 & -0.38752500 & 1.69358300 \\ \mathrm{H} & 3.37982800 & 1.25167800 & 1.94855100 \\ \mathrm{H} & 4.20539600 & 1.23909800 & -0.32417100 \\ \mathrm{H} & 3.71154300 & 0.76613600 & -1.94251800 \\ \mathrm{C} & 3.97820800 & 2.89446300 & -1.69718100 \\ \mathrm{H} & 3.87100200 & 3.66954000 & -0.92958800 \\ \mathrm{H} & 5.02470000 & 2.88605500 & -2.02011900 \\ \mathrm{H} & 3.36444200 & 3.18997700 & -2.55557100 \\ \mathrm{C} & 3.35504500 & 0.02914400 & 3.72786100 \\ \mathrm{H} & 2.62427100 & 0.63468900 & 4.27585000 \\ \mathrm{H} & 3.21014400 & -1.01580100 & 4.02503300 \\ \mathrm{H} & 4.35499300 & 0.33444700 & 4.05398900 \\ \mathrm{C} & 3.85651800 & -3.22270200 & -1.46961200 \\ \mathrm{H} & 3.13877100 & -4.04708800 & -1.39039600 \\ \mathrm{H} & 3.91253200 & -2.93040600 & -2.52454000 \\ \mathrm{H} & 4.83962600 & -3.60587900 & -1.17626200\end{array}$

$\left(\mathrm{RNH}_{2}\right) \mathrm{Cd}(\mathrm{OA})_{2}$

\begin{tabular}{|c|c|c|c|}
\hline & 1.2 & $\begin{array}{c}1.019 \\
2.365\end{array}$ & 6 Et \\
\hline $\mathrm{Cd}$ & -0.02200700 & -0.08170200 & -0.014272 \\
\hline $\mathrm{O}$ & 2.06434000 & -0.77490400 & 0.8728350 \\
\hline $\mathrm{O}$ & 1.78051000 & -0.47494800 & -1.30801300 \\
\hline $\mathrm{C}$ & 2.51649500 & -0.79879800 & -0.31069500 \\
\hline $\mathrm{C}$ & 3.94778500 & -1.22400700 & -0.59486100 \\
\hline $\mathrm{H}$ & 3.88447000 & -2.11789700 & -1.22842700 \\
\hline $\mathrm{H}$ & 4.39 & -0.45020900 & 8200 \\
\hline $\mathrm{C}$ & 4.80368800 & -1.49090500 & 0.64373600 \\
\hline $\mathrm{H}$ & 5.80942300 & -1.80959600 & 0.34935300 \\
\hline $\mathrm{H}$ & 4.89726700 & -0.59342700 & 1.26278000 \\
\hline $\mathrm{H}$ & 4.36180200 & -2.27295500 & 1.26724900 \\
\hline $\mathrm{O}$ & -1.92481300 & -1.21433600 & 0.52503000 \\
\hline $\mathrm{O}$ & -2.09637100 & 0.40347500 & -0.98477800 \\
\hline $\mathrm{C}$ & -2.62132000 & -0.52728500 & -0.29406400 \\
\hline $\mathrm{C}$ & -4.10055300 & -0.82727200 & -0.47456600 \\
\hline $\mathrm{H}$ & -4.20715100 & -1.23144700 & -1.48990700 \\
\hline $\mathrm{H}$ & -4.62696100 & 0.13393500 & -0.48821300 \\
\hline $\mathrm{C}$ & -4.70285700 & -1.78445700 & 0.55521700 \\
\hline $\mathrm{H}$ & -4.6279 & 1.37660800 & 1.568 \\
\hline
\end{tabular}




$\begin{array}{rrrr}\mathrm{H} & -5.76144600 & -1.96106400 & 0.33629700 \\ \mathrm{H} & -4.18267000 & -2.74591300 & 0.55085200 \\ \mathrm{C} & 0.66596100 & 3.46405000 & -0.62351700 \\ \mathrm{H} & 0.73481600 & 2.64201700 & -1.34365500 \\ \mathrm{H} & 1.66590100 & 3.63905000 & -0.20879100 \\ \mathrm{H} & 0.36958100 & 4.36488700 & -1.16939100 \\ \mathrm{C} & -0.34774600 & 3.16273200 & 0.47544800 \\ \mathrm{H} & -0.42708200 & 4.01781400 & 1.16011700 \\ \mathrm{H} & -1.33528400 & 2.98289600 & 0.04112800 \\ \mathrm{~N} & 0.02719700 & 1.93196600 & 1.22510700 \\ \mathrm{H} & 0.96382000 & 2.02110800 & 1.61880300 \\ \mathrm{H} & -0.60371300 & 1.79669000 & 2.01383000\end{array}$

$\left(\mathrm{SePPh}_{2} \mathrm{H}\right)_{2} \mathrm{Cd}(\mathrm{OA})_{2}$

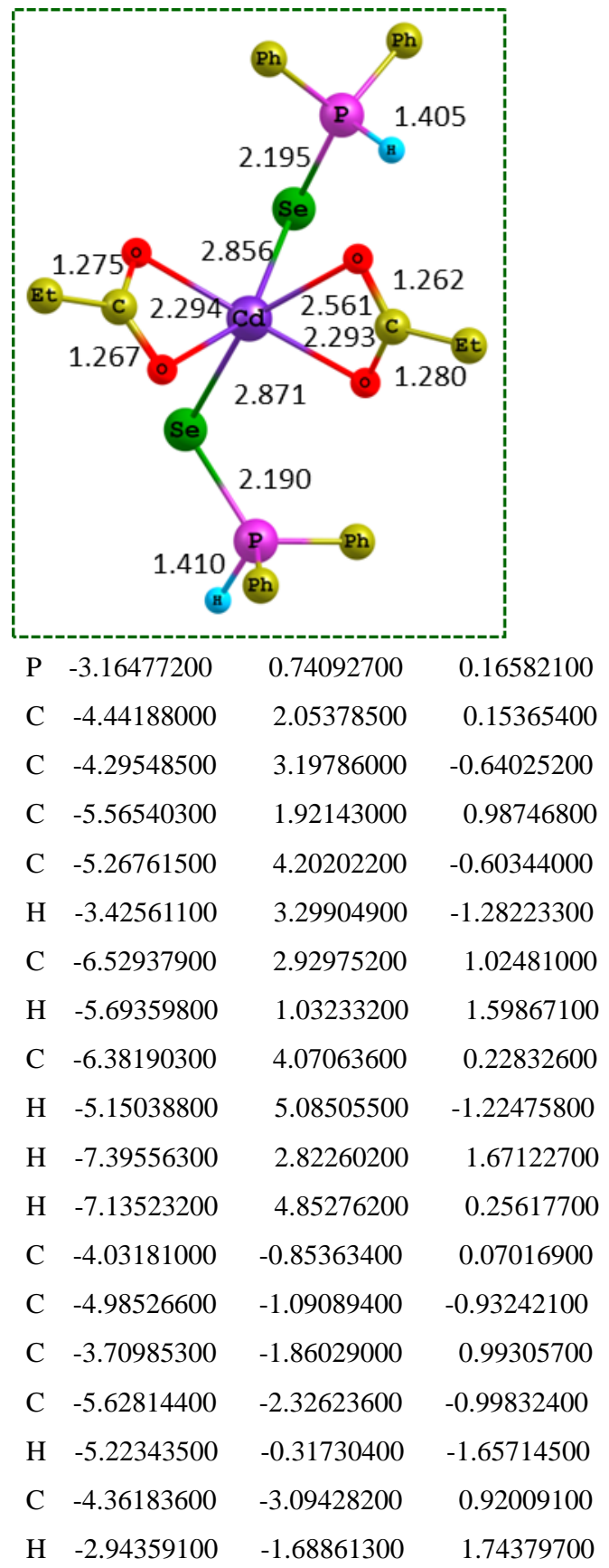

\begin{tabular}{|c|c|c|c|}
\hline $\mathrm{C}$ & -5.31962200 & -3.32688700 & -0.06922600 \\
\hline $\mathrm{H}$ & -6.36608700 & -2.50968600 & -1.77374500 \\
\hline $\mathrm{H}$ & -4.11152300 & -3.87475600 & 1.63251500 \\
\hline $\mathrm{H}$ & -5.82078700 & -4.28914800 & -0.12412800 \\
\hline & -1.56846100 & 1.09785200 & -1.29834200 \\
\hline $\mathrm{H}$ & -2.64010400 & 0.72168900 & 1.46938000 \\
\hline $\mathrm{P}$ & 3.94244600 & 0.56872400 & -1.21476500 \\
\hline $\mathrm{C}$ & 3.87368700 & 2.28994400 & -0.62467300 \\
\hline $\mathrm{C}$ & 4.53764200 & 3.29060700 & -1.35142700 \\
\hline $\mathrm{C}$ & 3.13585700 & 2.62145200 & 0.52343300 \\
\hline $\mathrm{C}$ & 4.47437900 & 4.61996100 & -0.92690000 \\
\hline $\mathrm{H}$ & 5.10175200 & 3.03925100 & -2.24597200 \\
\hline $\mathrm{C}$ & 3.07988300 & 3.95319600 & 0.93841500 \\
\hline $\mathrm{H}$ & 2.60081500 & 1.85409600 & 1.07901800 \\
\hline $\mathrm{C}$ & 3.74576700 & 4.95104300 & 0.21874300 \\
\hline $\mathrm{H}$ & 4.99098100 & 5.39151300 & -1.49031100 \\
\hline $\mathrm{H}$ & 2.50880200 & 4.21158600 & 1.82539400 \\
\hline $\mathrm{H}$ & 3.69484300 & 5.98483400 & 0.54864900 \\
\hline $\mathrm{C}$ & 4.65232300 & -0.45949300 & 0.11628900 \\
\hline $\mathrm{C}$ & 4.09347300 & -1.69980600 & 0.44214500 \\
\hline $\mathrm{C}$ & 5.79239500 & 0.00538500 & 0.79642600 \\
\hline $\mathrm{C}$ & 4.67535100 & -2.47357800 & 1.45150400 \\
\hline $\mathrm{H}$ & 3.20269700 & -2.06637400 & -0.05753400 \\
\hline $\mathrm{C}$ & 6.37133800 & -0.77813400 & 1.79399600 \\
\hline $\mathrm{H}$ & 6.22044900 & 0.97575400 & 0.55950000 \\
\hline $\mathrm{C}$ & 5.81126600 & -2.01890900 & 2.12282100 \\
\hline $\mathrm{H}$ & 4.22303300 & -3.42629700 & 1.70851600 \\
\hline $\mathrm{H}$ & 7.25245200 & -0.41874600 & 2.31779600 \\
\hline $\mathrm{H}$ & 6.25975000 & -2.62404000 & 2.90587500 \\
\hline $\mathrm{Se}$ & 2.16941800 & -0.21270300 & -2.23576800 \\
\hline $\mathrm{H}$ & 4.96467200 & 0.66016400 & -2.18115600 \\
\hline $\mathrm{Cd}$ & 0.26389700 & -0.77652600 & -0.16396900 \\
\hline $\mathrm{O}$ & 1.21384800 & 0.25039200 & 1.65263300 \\
\hline $\mathrm{O}$ & -0.82758200 & -0.49066100 & 2.13485600 \\
\hline $\mathrm{C}$ & 0.26040700 & 0.04592700 & 2.48128900 \\
\hline $\mathrm{C}$ & 0.49736300 & 0.46863800 & 3.92793100 \\
\hline $\mathrm{H}$ & 1.36063800 & -0.10863500 & 4.28257800 \\
\hline $\mathrm{H}$ & 0.83217700 & 1.51324900 & 3.91197400 \\
\hline $\mathrm{C}$ & -0.69826100 & 0.28760000 & 4.86373600 \\
\hline $\mathrm{H}$ & -0.43652700 & 0.58940300 & 5.88407000 \\
\hline $\mathrm{H}$ & -1.55177300 & 0.89173700 & 4.53978200 \\
\hline $\mathrm{H}$ & -1.02491400 & -0.75591100 & 4.88580500 \\
\hline $\mathrm{O}$ & 1.16568600 & -3.01625000 & 0.25329800 \\
\hline $\mathrm{O}$ & -0.72578100 & -2.72029700 & -0.87446100 \\
\hline $\mathrm{C}$ & 0.17189700 & -3.47958800 & -0.38052400 \\
\hline $\mathrm{C}$ & 0.04569500 & -4.99081700 & -0.54107700 \\
\hline
\end{tabular}

$\begin{array}{llll}\text { C } & -5.31962200 & -3.32688700 & -0.06922600\end{array}$

H $\quad-4.11152300 \quad-3.87475600 \quad 1.63251500$

$\mathrm{H} \quad-5.82078700 \quad-4.28914800 \quad-0.12412800$

$\mathrm{H} \quad-2.64010400 \quad 0.72168900 \quad 1.46938000$

$\begin{array}{llll}\mathrm{P} & 3.94244600 & 0.56872400 & -1.21476500\end{array}$

$\begin{array}{llll}\text { C } & 3.87368700 & 2.28994400 & -0.62467300\end{array}$

C $4.53764200 \quad 3.29060700 \quad-1.35142700$

C $\quad 4.47437900-4.61996100-0.92690000$

H $\quad 5.10175200 \quad 3.03925100 \quad-2.24597200$

$\mathrm{H} \quad 2.60081500 \quad 1.85409600 \quad 1.07901800$

$\begin{array}{llll}\text { C } & 3.74576700 & 4.95104300 & 0.21874300\end{array}$

H $4.99098100 \quad 5.39151300-1.49031100$

$-1.82539400$

$\begin{array}{llll}\text { C } & 4.65232300 & -0.45949300 & 0.11628900\end{array}$

$\begin{array}{llll}\text { C } & 4.09347300 & -1.69980600 & 0.44214500\end{array}$

$\begin{array}{llll}\text { C } & 4.67535100 & -2.47357800 & 1.45150400\end{array}$

$\begin{array}{llll}\mathrm{H} & 3.20269700 & -2.06637400 & -0.05753400\end{array}$

$\begin{array}{llll}\text { C } & 6.37133800 & -0.77813400 & 1.79399600\end{array}$

$\begin{array}{llll}\mathrm{H} & 6.22044900 & 0.97575400 & 0.55950000\end{array}$

$\begin{array}{llll}\text { C } & 5.81126600 & -2.01890900 & 2.12282100\end{array}$

H $4.22303300--3.42629700-1.70851600$

$\begin{array}{llll}\mathrm{H} & 7.25245200 & -0.41874600 & 2.31779600 \\ \mathrm{H} & 6.25975000 & -2.62404000 & 2.90587500\end{array}$

$\begin{array}{llll}\text { Se } & 2.16941800 & -0.21270300 & -2.23576800\end{array}$

$\begin{array}{llll}\mathrm{H} & 4.96467200 & 0.66016400 & -2.18115600\end{array}$

$\begin{array}{llll}\text { Cd } & 0.26389700 & -0.77652600 & -0.16396900\end{array}$

$\begin{array}{llll}\text { O } & 1.21384800 & 0.25039200 & 1.65263300\end{array}$

$\begin{array}{llll}\text { O } & -0.82758200 & -0.49066100 & 2.13485600\end{array}$

C $-0.26040700-0.04592700-2.48128900$

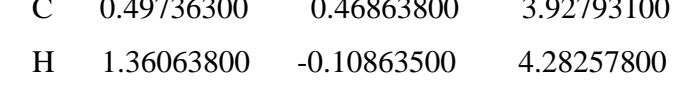

$\begin{array}{llll}\mathrm{H} & 0.83217700 & 1.51324900 & 3.91197400\end{array}$

$\begin{array}{llll}\text { C } & -0.69826100 & 0.28760000 & 4.86373600\end{array}$

$\begin{array}{llll}\mathrm{H} & -0.43652700 & 0.58940300 & 5.88407000\end{array}$

$\begin{array}{llll}\mathrm{H} & -1.55177300 & 0.89173700 & 4.53978200\end{array}$

$\begin{array}{llll}\text { O } & 1.16568600 & -3.01625000 & 0.25329800\end{array}$

$\begin{array}{lllll}\text { O } & -0.72578100 & -2.72029700 & -0.87446100\end{array}$

$\begin{array}{llll}\text { C } & 0.04569500 & -4.99081700 & -0.54107700\end{array}$ 


$$
\begin{array}{crrr}
\mathrm{H} & -0.07778200 & -5.40160200 & 0.46975700 \\
\mathrm{H} & 1.01839900 & -5.35848400 & -0.88873000 \\
\mathrm{C} & -1.08423100 & -5.45836000 & -1.45996100 \\
\mathrm{H} & -0.94866900 & -5.07710600 & -2.47661200 \\
\mathrm{H} & -1.11442400 & -6.55318700 & -1.50515500 \\
\mathrm{H} & -2.05372000 & -5.09722200 & -1.10563900
\end{array}
$$

$\left(\mathrm{HPPh}_{2}\right)_{2} \mathrm{Cd}(\mathrm{OA})_{2}$

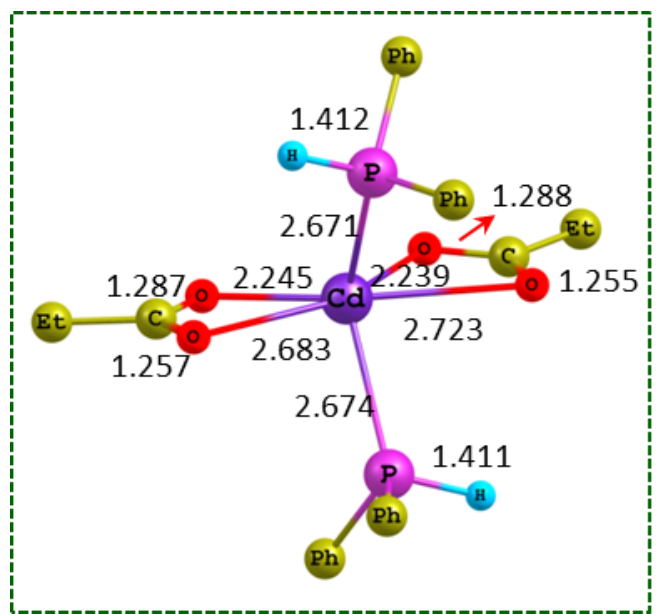

\begin{tabular}{lrrr}
$\mathrm{Cd}$ & -0.00829900 & -1.03949700 & 0.07321900 \\
$\mathrm{O}$ & -1.11531000 & -0.48205400 & -2.35077100 \\
$\mathrm{O}$ & -0.81773300 & -2.49280700 & -1.42639400 \\
$\mathrm{C}$ & -1.20871800 & -1.73321400 & -2.38996000 \\
$\mathrm{C}$ & -1.80322400 & -2.44295500 & -3.60426200 \\
$\mathrm{H}$ & -1.05243300 & -3.16308300 & -3.95279000 \\
$\mathrm{H}$ & -2.64027500 & -3.05231000 & -3.24071500 \\
$\mathrm{C}$ & -2.24940300 & -1.51971000 & -4.73824900 \\
$\mathrm{H}$ & -2.66135400 & -2.10337300 & -5.56919300 \\
$\mathrm{H}$ & -3.01714700 & -0.81863400 & -4.39748700 \\
$\mathrm{H}$ & -1.41179900 & -0.92628200 & -5.11705200 \\
$\mathrm{O}$ & 1.10373200 & -0.35235200 & 2.41529600 \\
$\mathrm{O}$ & 0.82672800 & -2.42280000 & 1.63199300 \\
$\mathrm{C}$ & 1.21815900 & -1.59766900 & 2.53820700 \\
$\mathrm{C}$ & 1.83108000 & -2.21405300 & 3.79279200 \\
$\mathrm{H}$ & 2.61650900 & -2.90494500 & 3.46301000 \\
$\mathrm{H}$ & 1.05745400 & -2.85008900 & 4.24196800 \\
$\mathrm{C}$ & 2.37117000 & -1.20877100 & 4.81039100 \\
$\mathrm{H}$ & 1.58407500 & -0.52852600 & 5.14869300 \\
$\mathrm{H}$ & 2.77801800 & -1.72868700 & 5.68499300 \\
$\mathrm{H}$ & 3.16732200 & -0.59600900 & 4.37636400 \\
$\mathrm{P}$ & -2.11227900 & 0.31444000 & 1.00938200 \\
$\mathrm{C}$ & -2.03736800 & 0.57348500 & 2.39568500 \\
$\mathrm{C}$ & -3.73771200 & -0.54076800 & 0.86998900 \\
\hline & -3.76120100 & -1.88697300 & 0.47445100 \\
\hline
\end{tabular}

$$
\begin{array}{lrrr}
\mathrm{C} & -4.97721200 & -2.57441100 & 0.39624900 \\
\mathrm{H} & -2.83988300 & -2.39903600 & 0.21009500 \\
\mathrm{C} & -6.15601700 & -0.57781400 & 1.08908600 \\
\mathrm{H} & -4.94246500 & 1.15809500 & 1.46166300 \\
\mathrm{C} & -6.17326400 & -1.92322100 & 0.70365500 \\
\mathrm{H} & -4.98403700 & -3.61597600 & 0.08844700 \\
\mathrm{H} & -7.08479000 & -0.06536800 & 1.32376700 \\
\mathrm{H} & -7.11682500 & -2.45770500 & 0.63885200 \\
\mathrm{C} & -2.36876200 & 2.01924400 & 0.38886300 \\
\mathrm{C} & -2.37697500 & 2.25026700 & -0.99838800 \\
\mathrm{C} & -2.53366500 & 3.09884800 & 1.27206900
\end{array}
$$$$
\begin{array}{llll}
\text { C } & -2.57189700 & 3.54466800 & -1.48531300
\end{array}
$$$$
\begin{array}{llll}
\mathrm{H} & -2.21477300 & 1.42585600 & -1.68843900
\end{array}
$$$$
\begin{array}{llll}
\text { C } & -2.71776100 & 4.39276400 & 0.77660900
\end{array}
$$$$
\begin{array}{llll}
\mathrm{H} & -2.51531500 & 2.93400600 & 2.34611400
\end{array}
$$$$
\text { C } \quad-2.74276600 \quad 4.61608600 \quad-0.60264100
$$$$
\begin{array}{llll}
\mathrm{H} & -2.57972200 & 3.71594600 & -2.55803800
\end{array}
$$$$
\mathrm{H} \quad-2.84204300 \quad 5.22162600 \quad 1.46779200
$$$$
\begin{array}{llll}
\mathrm{H} & -2.88865700 & 5.62133300 & -0.98794000
\end{array}
$$$$
\begin{array}{llll}
\mathrm{P} & 2.06523300 & 0.26462600 & -0.99795700
\end{array}
$$$$
\begin{array}{llll}
\mathrm{H} & 1.92351100 & 0.47252500 & -2.38714200
\end{array}
$$$$
\begin{array}{llll}
\text { C } & 3.68482800 & -0.61120300 & -0.91411400
\end{array}
$$$$
\text { C } \quad 4.87683200 \quad-0.00590000 \quad-1.34674700
$$$$
\begin{array}{llll}
\text { C } & 3.71718500 & -1.92804300 & -0.43007600
\end{array}
$$$$
\begin{array}{llll}
\text { C } & 6.07974800 & -0.71187300 & -1.30506700
\end{array}
$$$$
\mathrm{H} \quad 4.86838600 \quad 1.01872900 \quad-1.70757900
$$$$
\begin{array}{llll}
\text { C } & 4.92534000 & -2.63262700 & -0.39190500
\end{array}
$$$$
\text { H } 2.80974600 \quad-2.40496000 \quad-0.06943100
$$$$
\begin{array}{llll}
\text { C } & 6.10507600 & -2.02813700 & -0.82967100
\end{array}
$$$$
\begin{array}{llll}
\mathrm{H} & 6.99629600 & -0.23563100 & -1.64187500
\end{array}
$$$$
\text { H } 4.93890100 \quad-3.65115500 \quad-0.01498900
$$$$
\begin{array}{llll}
\mathrm{H} & 7.04254900 & -2.57613200 & -0.79738500
\end{array}
$$$$
\begin{array}{llll}
\text { C } & 2.37831600 & 1.98733400 & -0.45637100
\end{array}
$$$$
\begin{array}{llll}
\text { C } & 2.40114800 & 2.28124800 & 0.91864500
\end{array}
$$$$
\begin{array}{llll}
\text { C } & 2.57373700 & 3.01996600 & -1.38853600
\end{array}
$$$$
\begin{array}{llll}
\text { C } & 2.64156800 & 3.58936200 & 1.34531200
\end{array}
$$$$
\mathrm{H} \quad 2.21501800 \quad 1.49519300 \quad 1.64636800
$$$$
\begin{array}{llll}
\text { C } & 2.80476900 & 4.32782600 & -0.95350300
\end{array}
$$$$
\mathrm{H} \quad 2.54131900 \quad 2.80774500 \quad-2.45391100
$$$$
\begin{array}{llll}
\text { C } & 2.84433000 & 4.61280100 & 0.41406200
\end{array}
$$$$
\text { H } 2.66010800 \quad 3.80903100 \quad 2.40904400
$$$$
\mathrm{H} \quad 2.95259700 \quad 5.11990700 \quad-1.68221200
$$$$
\begin{array}{llll}
\mathrm{H} & 3.02567700 & 5.62914300 & 0.75237000
\end{array}
$$ 
$\left(\mathrm{SePR}_{3}\right)_{2} \mathrm{Cd}(\mathrm{OA})_{2}$

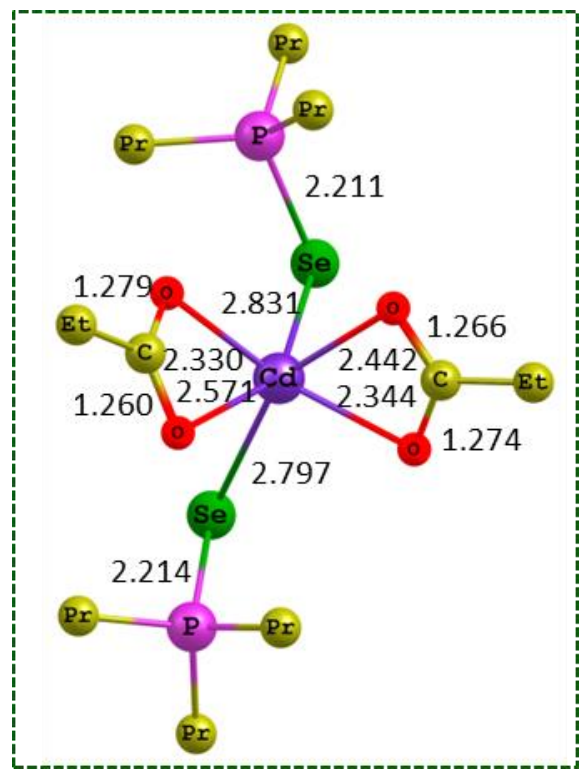

\begin{tabular}{|c|c|c|c|}
\hline 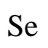 & 1.69094400 & -0.664 & 5900 \\
\hline $\mathrm{Se}$ & -2.16846000 & 0.49857700 & -2.02772600 \\
\hline $\mathrm{CH}$ & -0.08542600 & 0.50842200 & -0.11089400 \\
\hline & -0.96949200 & -0.96240000 & 1.46554700 \\
\hline & 1.17098600 & -0.51520700 & 1.88494600 \\
\hline & 0.07391800 & -1.04949500 & 2.20044000 \\
\hline & -0.05404100 & -1.85680800 & 3.49182800 \\
\hline & -1.01139500 & -1.58652100 & 3.95141300 \\
\hline & -0.15493700 & -2.91061900 & 3.19711600 \\
\hline C & 1.09866200 & -1.68325300 & 4.48210200 \\
\hline 1 & 0.93937300 & -2.30237400 & 5.37224500 \\
\hline $\mathrm{H}$ & 2.05329800 & -1.96772700 & 4.03076800 \\
\hline 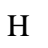 & 1.18773100 & -0.64054500 & 4.80240400 \\
\hline & -0.99863700 & 2.36543900 & 1.18564600 \\
\hline $\mathrm{O}$ & 0.86824700 & 2.64565100 & 0.01248700 \\
\hline $\mathrm{r}$ & -0.04764700 & 3.09467900 & 0.77647600 \\
\hline $\mathrm{C}$ & -0.02142400 & 4.55758700 & 1.21035500 \\
\hline $\mathrm{H}$ & -0.10917100 & 4.56929200 & 2.30359600 \\
\hline $\mathrm{H}$ & -0.95070700 & 5.00785200 & 0.83835500 \\
\hline $\mathrm{C}$ & 1.19332900 & 5.36188800 & 0.74545400 \\
\hline$H$ & 1.27584700 & 5.35475400 & -0.34516800 \\
\hline$H$ & 1.11691800 & 6.40280900 & 1.08040600 \\
\hline 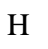 & 2.12111600 & 4600 & 76300 \\
\hline D & 3.57477000 & -0.56389300 & -0.76582700 \\
\hline $\mathrm{C}$ & 3.70935700 & -2.04414000 & 0.33085300 \\
\hline$C$ & 4.94173400 & -0.59311000 & -2.01701900 \\
\hline $\mathrm{C}$ & 3.68391200 & 0.96565800 & 0.25806800 \\
\hline 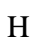 & 4.57142600 & -1.24731200 & -2.81461000 \\
\hline $\mathrm{H}$ & 4.97045900 & 0.41686600 & -2.44457200 \\
\hline H & 3.10946100 & 1.72615400 & -0.28157900 \\
\hline
\end{tabular}

\begin{tabular}{|c|c|c|c|}
\hline & 00 & 00 & 1.16108400 \\
\hline & .86920200 & .90089100 & 5994 \\
\hline & 2.70373800 & -2.14220000 & 0.75278100 \\
\hline & 6.34641600 & -1.04591300 & -1.58289300 \\
\hline & 3200 & -2.02407200 & 1.47507000 \\
\hline & 0 & 1.501032 & 70900 \\
\hline & 5.63123100 & 1.74643200 & -0.31196900 \\
\hline & .67455300 & 0.74121600 & 1.13100600 \\
\hline & 6.71863900 & -0.4 & 0400 \\
\hline & 6.29958500 & -2.0716 & 800 \\
\hline & 5.75026800 & -1.84488600 & 1.09525300 \\
\hline & 4.50112700 & -1.199 & 2.15627400 \\
\hline & 300 & -3.3 & 7400 \\
\hline & 4.98727100 & -4.19212400 & 27900 \\
\hline & 5.43541300 & -3.3 & 0400 \\
\hline & 15900 & -3.53953300 & 55400 \\
\hline & 7.33805100 & -0.99133600 & -2.75375300 \\
\hline & 7.01226000 & -1.63320000 & 17700 \\
\hline & 7.43617700 & 700 & -3 \\
\hline & 8.33229700 & -1.32710900 & 4500 \\
\hline & 4.98 & 00 & 400 \\
\hline $\mathrm{H}$ & 0 & 0 & 00 \\
\hline H & 3400 & 1000 & 6800 \\
\hline & 0 & 0 & 0 \\
\hline $\mathrm{P}$ & -3.77921200 & -0.24463000 & -0.70761500 \\
\hline & -3.94441100 & 0.81 & 5800 \\
\hline $\mathrm{C}$ & -5.35817200 & -0.14102700 & -1.67258700 \\
\hline C & -3.46180900 & -2.01053200 & -0.26 \\
\hline $\mathrm{H}$ & -5.27659800 & 0.78 & -2.24562000 \\
\hline & -5.323 & -0.95658500 & -2.4 \\
\hline $\mathrm{H}$ & -3.03535000 & -2.43804700 & -1.18045900 \\
\hline $\mathrm{H}$ & -2.64908800 & -1.97546900 & 0.47024600 \\
\hline $\mathrm{H}$ & 900 & 100 & 0.47734700 \\
\hline H & -2.94047400 & 1.19809800 & 1.00623200 \\
\hline C & -( & -0.15909100 & 0 \\
\hline C & -4.53076800 & 0.17784600 & 2.06806300 \\
\hline C & -4.62799100 & -2.88593500 & 0.22650500 \\
\hline $\mathrm{H}$ & -5.41088600 & -2.93814100 & -0.53960200 \\
\hline $\mathrm{H}$ & -5.08820500 & -2.45155600 & .12008400 \\
\hline $\mathrm{H}$ & -6.77174100 & -1.07655400 & -0.30102400 \\
\hline $\mathrm{H}$ & -6.70274300 & 0.67335700 & -0.17950600 \\
\hline 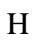 & -5.534 & -0.22381900 & 1.88263700 \\
\hline $\mathrm{H}$ & -3.8 & 00 & 0 \\
\hline $\mathrm{C}$ & -4.59280100 & 1.20033100 & 3.21166200 \\
\hline $\mathrm{H}$ & -5.24896700 & 2.04137000 & 2.95821400 \\
\hline & 800 & 300 & 4. \\
\hline
\end{tabular}

C $\quad-4.53076800$
0.74681500

$-0.33599400$

$-1.58289300$

0.60670900

0.31196900

00600

.19953800

2.25907400

61727900

2.67755400

$-2.75375300$

$-3.14302400$

$-2.44054500$

0.97549000

.42686800

71996900

0.79665800

67258700

2.24562000

2.40569100

0.47024600

0.47734700

89224400

2.06806300

0.53960200

17950600

37065000

12750200 


$\begin{array}{lrrr}\mathrm{H} & -3.59822800 & 1.60543700 & 3.42438300 \\ \mathrm{C} & -7.89274800 & -0.04464000 & -1.83265900 \\ \mathrm{H} & -7.85339000 & 0.88104000 & -2.41807800 \\ \mathrm{H} & -7.92834500 & -0.88309800 & -2.53776900 \\ \mathrm{H} & -8.83043100 & -0.04342300 & -1.26687100 \\ \mathrm{C} & -4.14800300 & -4.30902300 & 0.54749700 \\ \mathrm{H} & -3.70654100 & -4.78594000 & -0.33504800 \\ \mathrm{H} & -3.38751600 & -4.29922900 & 1.33586900 \\ \mathrm{H} & -4.97955500 & -4.93589800 & 0.88738700\end{array}$

$\left(\mathrm{PR}_{3}\right)_{2} \mathrm{Cd}(\mathrm{OA})_{2}$

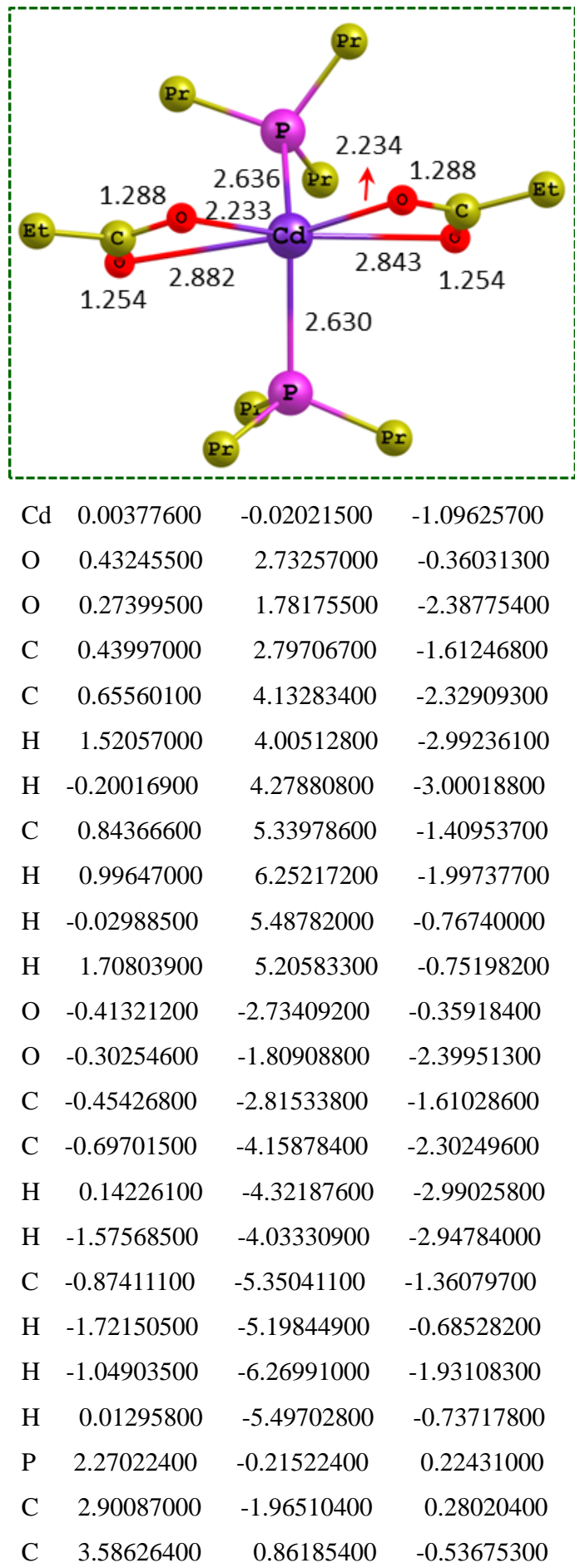

\begin{tabular}{|c|c|c|c|}
\hline $\mathrm{C}$ & 2.00663700 & 0.37553100 & 1.97043000 \\
\hline $\mathrm{H}$ & 3.41865200 & 0.79914900 & -1.61926900 \\
\hline $\mathrm{H}$ & 3.31171400 & 1.88696500 & -0.25758700 \\
\hline $\mathrm{H}$ & 1.31102800 & 1.21683300 & 1.86367900 \\
\hline $\mathrm{H}$ & 1.44974800 & -0.42750400 & 2.47214600 \\
\hline $\mathrm{H}$ & 3.33431800 & -2.14800800 & -0.71304200 \\
\hline $\mathrm{H}$ & 1.99259500 & -2.57864300 & 0.32337300 \\
\hline $\mathrm{C}$ & 5.07093000 & 0.59142600 & -0.23957500 \\
\hline C & 3.88154900 & -2.40611200 & 1.37977600 \\
\hline $\mathrm{C}$ & 3.19937800 & 0.81843500 & 2.83521600 \\
\hline $\mathrm{H}$ & 3.72195900 & 1.64410300 & 2.33841400 \\
\hline $\mathrm{H}$ & 3.92526700 & 0.00513500 & 2.94027900 \\
\hline $\mathrm{H}$ & 5.26406500 & 0.64985100 & 0.83700600 \\
\hline $\mathrm{H}$ & 5.32829000 & -0.42930300 & -0.54739400 \\
\hline $\mathrm{H}$ & 4.78106100 & -1.78126200 & 1.37118600 \\
\hline $\mathrm{H}$ & 3.41438900 & -2.26735700 & 2.36229500 \\
\hline $\mathrm{C}$ & 4.28254600 & -3.87994800 & 1.22167400 \\
\hline $\mathrm{H}$ & 4.78977400 & -4.05166200 & 0.26502200 \\
\hline $\mathrm{H}$ & 4.96296300 & -4.19247300 & 2.02145800 \\
\hline $\mathrm{H}$ & 3.40337600 & -4.53338700 & 1.25312800 \\
\hline $\mathrm{C}$ & 5.98541500 & 1.58154200 & -0.97543900 \\
\hline $\mathrm{H}$ & 5.83763200 & 1.52513300 & -2.06010700 \\
\hline $\mathrm{H}$ & 5.78258200 & 2.61344500 & -0.66636300 \\
\hline $\mathrm{H}$ & 7.04083000 & 1.37158100 & -0.77014700 \\
\hline $\mathrm{C}$ & 2.74663400 & 1.28013500 & 4.22784800 \\
\hline $\mathrm{H}$ & 2.04222300 & 2.11683100 & 4.15609900 \\
\hline $\mathrm{H}$ & 2.24812300 & 0.46920800 & 4.77204900 \\
\hline $\mathrm{H}$ & 3.59937100 & 1.61140400 & 4.83042500 \\
\hline $\mathrm{P}$ & -2.25667900 & 0.20239500 & 0.24113400 \\
\hline $\mathrm{C}$ & -3.60882800 & -0.77772900 & -0.58643500 \\
\hline $\mathrm{C}$ & -2.04885500 & -0.47676200 & 1.96229800 \\
\hline $\mathrm{C}$ & -2.81249200 & 1.97467400 & 0.36301500 \\
\hline $\mathrm{H}$ & -1.39398700 & -1.34645300 & 1.82836000 \\
\hline $\mathrm{H}$ & -1.46080300 & 0.27481900 & 2.50649400 \\
\hline $\mathrm{H}$ & -1.87795500 & 2.54571700 & 0.42482600 \\
\hline $\mathrm{H}$ & -3.23632100 & 2.21168600 & -0.62298100 \\
\hline $\mathrm{H}$ & -3.39046600 & -1.82719000 & -0.35063800 \\
\hline $\mathrm{H}$ & -3.42024100 & -0.67466400 & -1.66229600 \\
\hline $\mathrm{C}$ & -3.27693600 & -0.89265800 & 2.78996400 \\
\hline $\mathrm{C}$ & -5.08217100 & -0.44660200 & -0.29608200 \\
\hline $\mathrm{C}$ & -3.77470600 & 2.42050900 & 1.47677300 \\
\hline $\mathrm{H}$ & -3.32253900 & 2.21683400 & 2.45512800 \\
\hline $\mathrm{H}$ & -4.70667100 & 1.84604300 & 1.43839000 \\
\hline $\mathrm{H}$ & -3.96398500 & -0.04889900 & 2.91291200 \\
\hline $\mathrm{H}$ & -3.83017500 & -1.67243800 & 2.25354300 \\
\hline $\mathrm{H}$ & -5.29680000 & -0.55537800 & 0.77241500 \\
\hline
\end{tabular}




$\begin{array}{rrrr}\mathrm{H} & -5.27915200 & 0.60247200 & -0.54753900 \\ \mathrm{C} & -6.03528400 & -1.34318700 & -1.09956300 \\ \mathrm{H} & -5.89166100 & -2.40035600 & -0.84785600 \\ \mathrm{H} & -7.08129700 & -1.08966900 & -0.89530800 \\ \mathrm{H} & -5.86809100 & -1.23297700 & -2.17718500 \\ \mathrm{C} & -2.87371800 & -1.42735100 & 4.17168700 \\ \mathrm{H} & -2.20518500 & -2.29115300 & 4.08072100 \\ \mathrm{H} & -2.35166100 & -0.66107200 & 4.75700400 \\ \mathrm{H} & -3.75242000 & -1.74310800 & 4.74461900 \\ \mathrm{C} & -4.09599200 & 3.91896300 & 1.37860500 \\ \mathrm{H} & -3.18400100 & 4.52298900 & 1.44352500 \\ \mathrm{H} & -4.58489100 & 4.15743900 & 0.42674000 \\ \mathrm{H} & -4.76615000 & 4.23309700 & 2.18643000\end{array}$

$\left(\mathrm{RNH}_{2}\right)_{2} \mathrm{Cd}(\mathrm{OA})_{2}$

\begin{tabular}{|c|c|c|c|}
\hline \multicolumn{4}{|c|}{ (1.284 } \\
\hline $\mathrm{Cd}$ & -0.04696900 & 0.12849700 & -0.0851770 \\
\hline $\mathrm{O}$ & -2.46838400 & -0.24527800 & 0.89423600 \\
\hline $\mathrm{O}$ & -1.75818100 & -0.83511700 & -1.13567400 \\
\hline $\mathrm{C}$ & -2.67045700 & -0.76789800 & -0.23514400 \\
\hline $\mathrm{C}$ & -4.05334400 & -1.32272600 & -0.56030900 \\
\hline $\mathrm{H}$ & -4.37401700 & -1.91200000 & 0.30658400 \\
\hline $\mathrm{H}$ & -4.73101300 & -0.45879100 & -0.59010700 \\
\hline $\mathrm{C}$ & -4.15436100 & -2.13265200 & -1.85391900 \\
\hline $\mathrm{H}$ & -5.18363800 & -2.47382000 & -2.01152200 \\
\hline $\mathrm{H}$ & -3.85113900 & -1.53552300 & -2.71817200 \\
\hline $\mathrm{H}$ & -3.50445600 & -3.01253700 & -1.82220900 \\
\hline $\mathrm{O}$ & 2.65138700 & 1.45959900 & -0.09034900 \\
\hline $\mathrm{O}$ & 1.91887300 & -0.52391500 & -0.85619600 \\
\hline $\mathrm{C}$ & 2.84191000 & 0.35161300 & -0.64558600 \\
\hline $\mathrm{C}$ & 4.24913100 & 0.00747100 & -1.13738900 \\
\hline $\mathrm{H}$ & 4.95301400 & 0.38035000 & -0.38523200 \\
\hline $\mathrm{H}$ & 4.41792600 & 0.62687200 & -2.02911100 \\
\hline $\mathrm{C}$ & 4.50108000 & -1.46647400 & -1.46153400 \\
\hline $\mathrm{H}$ & 3.80149900 & -1.82802400 & -2.21964300 \\
\hline $\mathrm{H}$ & 5.52212700 & -1.60817700 & -1.83341600 \\
\hline $\mathrm{H}$ & 4.37542100 & -2.09559800 & -0.57398400 \\
\hline
\end{tabular}

$\begin{array}{rrrr}\mathrm{C} & 0.92547000 & -2.71300800 & 1.94970700 \\ \mathrm{H} & 1.05456000 & -2.61915400 & 0.86674500 \\ \mathrm{H} & -0.08672800 & -3.08184300 & 2.15243100 \\ \mathrm{H} & 1.63302500 & -3.46701900 & 2.30919800 \\ \mathrm{C} & 1.18033600 & -1.38155600 & 2.64961400 \\ \mathrm{H} & 1.13001600 & -1.51291500 & 3.73954200 \\ \mathrm{H} & 2.18143800 & -1.01276100 & 2.40695200 \\ \mathrm{~N} & 0.21077900 & -0.34698000 & 2.20949500 \\ \mathrm{H} & -0.75251900 & -0.61886700 & 2.41391600 \\ \mathrm{H} & 0.38280000 & 0.52375300 & 2.70871800 \\ \mathrm{C} & -2.23466700 & 3.28577900 & 0.37036700 \\ \mathrm{H} & -2.58114800 & 2.27685600 & 0.61487800 \\ \mathrm{H} & -2.56889400 & 3.53137000 & -0.64522300 \\ \mathrm{H} & -2.71308200 & 3.99427800 & 1.05469100 \\ \mathrm{C} & -0.71619200 & 3.38794600 & 0.48445300 \\ \mathrm{H} & -0.38729300 & 4.41688500 & 0.28300000 \\ \mathrm{H} & -0.39508000 & 3.14532600 & 1.50327900 \\ \mathrm{~N} & -0.03133000 & 2.44321700 & -0.43116300 \\ \mathrm{H} & -0.27892900 & 2.65749100 & -1.39623000 \\ \mathrm{H} & 0.99145900 & 2.50899900 & -0.35264300\end{array}$

\section{C'-TS1}

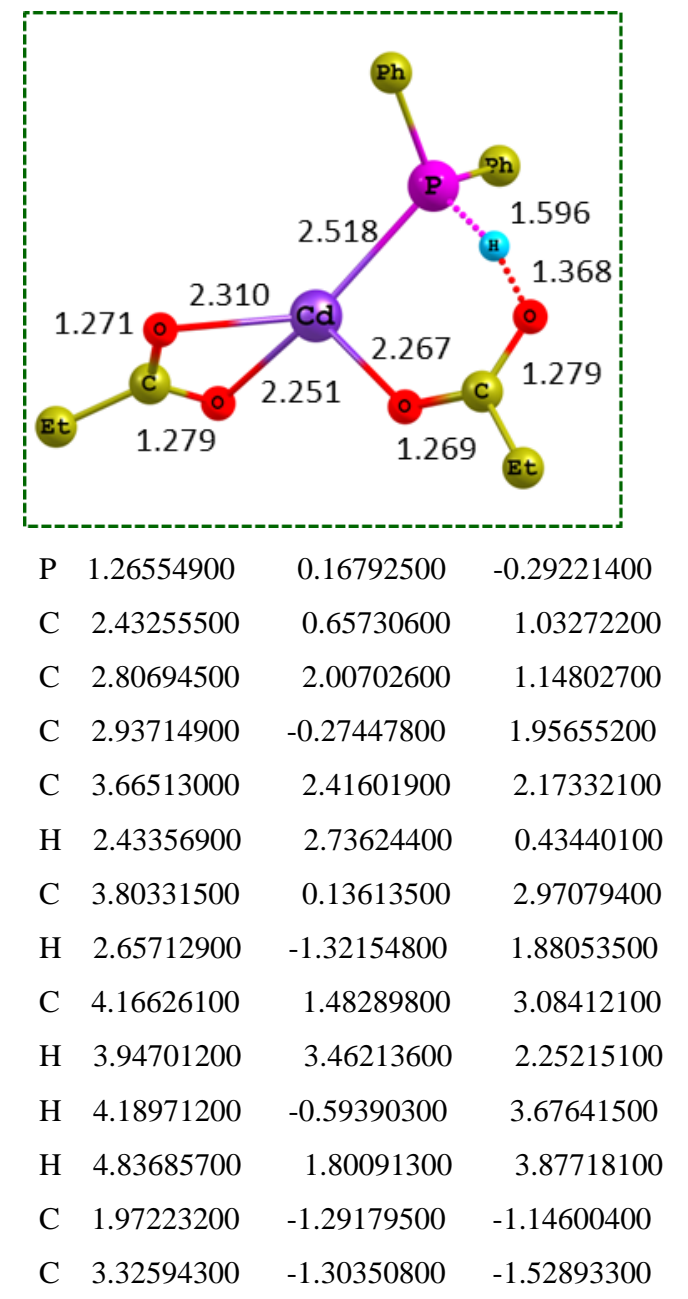




\begin{tabular}{|c|c|c|c|}
\hline $\mathrm{C}$ & 1.15860900 & -2.38453300 & -1.48704600 \\
\hline $\mathrm{C}$ & 3.85052900 & -2.38723500 & -2.23417000 \\
\hline $\mathrm{H}$ & 3.96970000 & -0.46663100 & -1.27382900 \\
\hline $\mathrm{C}$ & 1.68491000 & -3.46319500 & -2.20425000 \\
\hline $\mathrm{H}$ & 0.11340700 & -2.40364000 & -1.18943800 \\
\hline $\mathrm{C}$ & 3.03091200 & -3.46845500 & -2.57662300 \\
\hline $\mathrm{H}$ & 4.89827200 & -2.38469500 & -2.52118000 \\
\hline $\mathrm{H}$ & 1.04258300 & -4.30063200 & -2.46094900 \\
\hline & 3.44094600 & -4.30977200 & -3.12780800 \\
\hline & -1.16856300 & 0.00857100 & 0.33271800 \\
\hline & 0.91509700 & 1.43600000 & -1.19594500 \\
\hline & -1.62928300 & 3.82170900 & -2.25718600 \\
\hline & -1.09818100 & 4.72173500 & -1.92212000 \\
\hline & -1.39682400 & 3.73069700 & -3.32542300 \\
\hline & 0.29306100 & 2.50708400 & -1.77785600 \\
\hline & -0.96045200 & 2.63985300 & -1.56377700 \\
\hline & -1.67338000 & 1.88525700 & -0.83423400 \\
\hline & -3.13483200 & 3.95183500 & -2.02628800 \\
\hline & -3.36512600 & 4.06723600 & -0.96337500 \\
\hline & -3.52521600 & 4.82505400 & -2.55995200 \\
\hline & -3.66847300 & 3.06579700 & -2.38207500 \\
\hline & -2.82297500 & -1.58746600 & 0.10188100 \\
\hline & -2.69281000 & -0.36355600 & 1.94614500 \\
\hline & 3.26491100 & -1.27229800 & 1.25103100 \\
\hline & -4.49169900 & -1.95399500 & 1.82939900 \\
\hline & -5.25023200 & -1.17038500 & 1.95308000 \\
\hline & -4.23231900 & -2.26947900 & 2.84686000 \\
\hline & -5.03696800 & -3.11913400 & 1.00288200 \\
\hline & -4.29296400 & -3.91479600 & 0.90044500 \\
\hline & -5.92577200 & -3.54149700 & 1.48363100 \\
\hline & -5.31063200 & -2.79458400 & -0.00478000 \\
\hline
\end{tabular}

$\begin{array}{rrrr}\text { C } & 2.99067300 & 0.03655000 & 1.73283300 \\ \text { C } & 3.34108300 & 2.75266900 & 2.28424200 \\ \text { H } & 2.12931400 & 3.10659600 & 0.53995500 \\ \text { C } & 3.76859400 & 0.43566600 & 2.82056500 \\ \text { H } & 2.85925900 & -1.02218300 & 1.52848000 \\ \text { C } & 3.94284600 & 1.79535100 & 3.10379700 \\ \text { H } & 3.47681400 & 3.81097800 & 2.48960400 \\ \text { H } & 4.23346700 & -0.31599100 & 3.45284800 \\ \text { H } & 4.54518900 & 2.10317600 & 3.95370700\end{array}$

$\begin{array}{llll}\text { C } & 2.14029900 & -0.93230400 & -1.29093800\end{array}$

$\begin{array}{llll}\text { C } & 3.51926600 & -0.88479800 & -1.57358100\end{array}$

$\begin{array}{llll}\text { C } & 1.41831700 & -2.06670700 & -1.70087400\end{array}$

$\begin{array}{llll}\text { C } & 4.15303600 & -1.94092100 & -2.23026300\end{array}$

$\mathrm{H} \quad 4.10207100 \quad-0.01742400 \quad-1.27628900$

$\begin{array}{llll}\text { C } & 2.05063100 & -3.11757300 & -2.37244700\end{array}$

$\mathrm{H} \quad 0.35449200 \quad-2.14606800 \quad-1.49050700$

$\begin{array}{llll}\text { C } & 3.42101500 & -3.06164700 & -2.63630500\end{array}$

$\mathrm{H} \quad 5.21969400 \quad-1.88454800 \quad-2.43015400$

$\mathrm{H} \quad 1.46991800 \quad-3.98444600 \quad-2.67540900$

H $3.91438700 \quad-3.88211800 \quad-3.14931200$

Cd-0.85341500 $\quad-0.21283300 \quad 0.39748600$

$\begin{array}{llll}\mathrm{H} & 0.06134900 & 2.10110800 & -1.65277700\end{array}$

C $-2.87537400 \quad 3.40550400 \quad-2.18442500$

$\mathrm{H}-2.55125600 \quad 4.42864100 \quad-1.95532000$

$\mathrm{H}-2.80042500 \quad 3.31604100 \quad-3.27552700$

O $-0.61435700 \quad 2.72196500 \quad-2.04550100$

C $-1.84154800 \quad 2.47306300 \quad-1.59374400$

O $-2.11315800 \quad 1.58640100 \quad-0.78504500$

C $-4.29964700 \quad 3.14564300 \quad-1.69621000$

$\mathrm{H}-4.37298200 \quad 3.25631600 \quad-0.61082900$

$\mathrm{H}-4.99005700 \quad 3.85504600 \quad-2.16238400$

$\mathrm{H}-4.62547900 \quad 2.13252800 \quad-1.94744800$

O $-2.44477400 \quad-1.90106200 \quad 0.23357700$

O - $2.34754800 \quad-0.60083700 \quad 2.02661200$

C $-2.88647600 \quad-1.56138200 \quad 1.37394900$

C $-4.07488500 \quad-2.27231600 \quad 1.99621600$

$\mathrm{H}-4.87636200 \quad-1.52590000 \quad 2.07123400$

H -3.80098500 -2.50932800 3.03084800

C $-4.55292400 \quad-3.51452700 \quad 1.24332700$

H -3.76553200 -4.27231400 1.19010700

$\mathrm{H}-5.41855100 \quad-3.95516000 \quad 1.74939700$

H $-4.84022500 \quad-3.26817400 \quad 0.21746600$ 
C

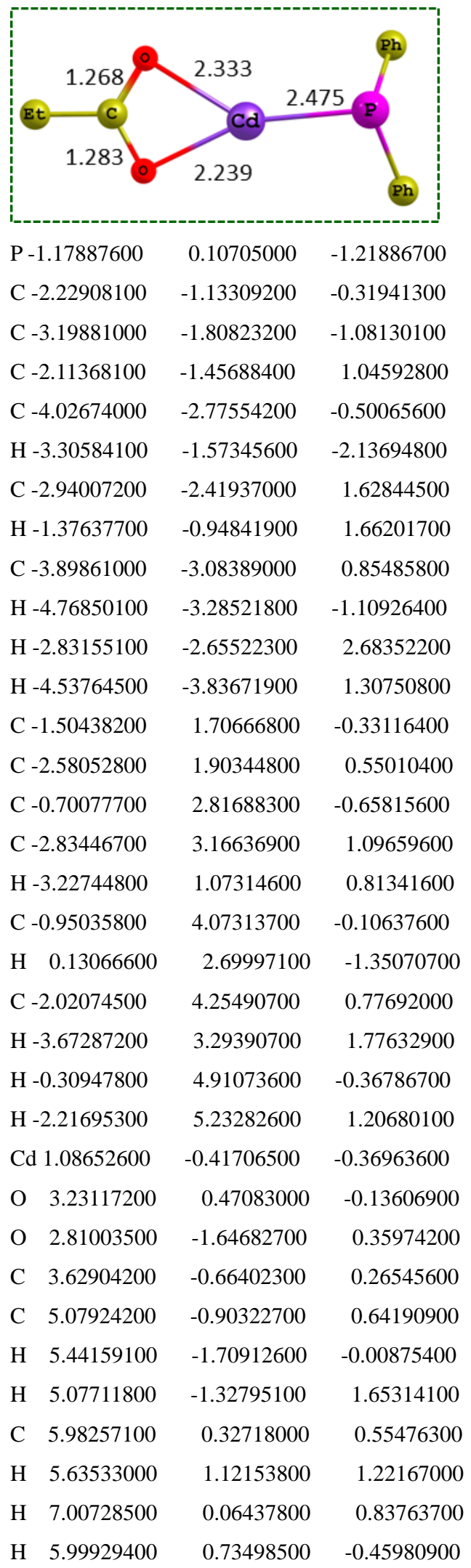

C"-TS1

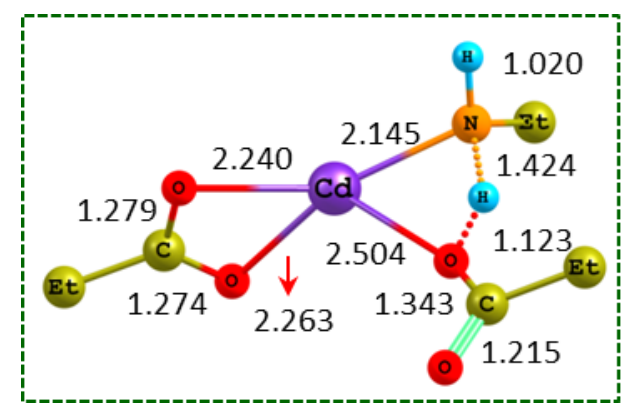

\begin{tabular}{|c|c|c|c|}
\hline $\mathrm{Cd}$ & 0.61598800 & 0.54558800 & -0.289 \\
\hline $\mathrm{C}$ & -1.46442000 & 2.77117400 & 0.55137200 \\
\hline $\mathrm{H}$ & -1.29350400 & 2.39399100 & 1.56702900 \\
\hline & -0.75596300 & 3.59824400 & 0.39953900 \\
\hline $\mathrm{C}$ & -2.89527300 & 3.30399700 & 0.43791900 \\
\hline $\mathrm{H}$ & -3.08755300 & 3.70389800 & -0.56516000 \\
\hline $\mathrm{H}$ & -3.62590500 & 2.51173700 & 0.63232200 \\
\hline $\mathrm{H}$ & -3.06553800 & 4.11305300 & 33400 \\
\hline $\mathrm{O}$ & -2.10043200 & -2.84699200 & 0.34218500 \\
\hline$C$ & -2.41988000 & -1.68541600 & 0.18780700 \\
\hline $\mathrm{C}$ & -3.86442500 & -1.21950600 & 0.02147100 \\
\hline $\mathrm{H}$ & -4.07694900 & -0.50598700 & 0.82910500 \\
\hline $\mathrm{H}$ & -3.92700000 & -0.64104800 & -0.91023600 \\
\hline$c$ & -4.88056000 & -2.36120700 & 0.02378000 \\
\hline$H$ & -5.89612000 & -1.96906100 & -0.09429700 \\
\hline $\mathrm{H}$ & -4.68288900 & -3.06337600 & -0.79087000 \\
\hline $\mathrm{H}$ & -4.83249200 & 569100 & 0.95886600 \\
\hline $\mathrm{N}$ & -1.21376700 & 1.66056300 & -0.39075500 \\
\hline $\mathrm{H}$ & -1.40560900 & 1.97692200 & -1.34103100 \\
\hline $\mathrm{O}$ & -1.50957000 & -0.69896700 & 0.16024600 \\
\hline $\mathrm{H}$ & -1.73923600 & 0.37308600 & -0.08319100 \\
\hline $\mathrm{O}$ & 2.34316200 & -0.46191800 & -1.29940400 \\
\hline 0 & 2.50539700 & 0.04086900 & 92000 \\
\hline $\mathrm{C}$ & 3.00451500 & -0.47106100 & -0.20508200 \\
\hline $\mathrm{C}$ & 4.37522100 & -1.11570200 & -0.18621000 \\
\hline $\mathrm{H}$ & 4.91824600 & -0.74001700 & -1.06093200 \\
\hline $\mathrm{H}$ & 4.21076600 & -2.18333800 & -0.38167900 \\
\hline$c$ & 5.17060400 & -0.91176000 & 1.10392800 \\
\hline $\mathrm{H}$ & 4.62884200 & -1.30503800 & 1.96814200 \\
\hline $\mathrm{H}$ & 6.13475100 & -1.42602400 & 1.03648500 \\
\hline $\mathrm{H}$ & 5.36197000 & 0.14945300 & 1.28909400 \\
\hline
\end{tabular}




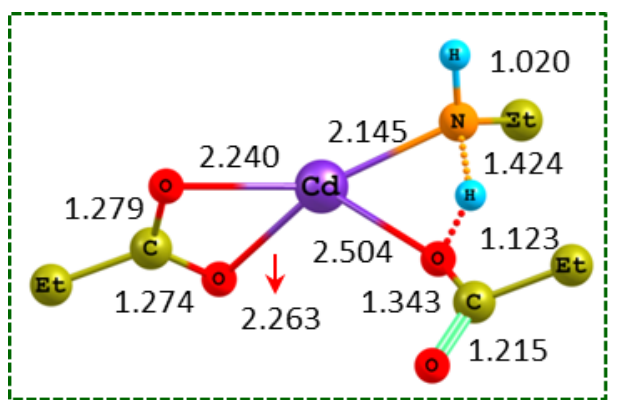

$\begin{array}{lrrr}\mathrm{Cd} & 0.70476000 & 0.62609900 & -0.36161400 \\ \mathrm{C} & -1.33994600 & 2.72531100 & 0.63801600 \\ \mathrm{H} & -1.13891700 & 2.30554100 & 1.63144000 \\ \mathrm{H} & -0.64083900 & 3.56550300 & 0.50629900 \\ \mathrm{C} & -2.77359400 & 3.26210300 & 0.59519800 \\ \mathrm{H} & -2.99927100 & 3.70133200 & -0.38428700 \\ \mathrm{H} & -3.49780600 & 2.46201700 & 0.78153200 \\ \mathrm{H} & -2.91869000 & 4.04206600 & 1.35162900 \\ \mathrm{O} & -2.40701900 & -2.92663700 & 0.24487300 \\ \mathrm{C} & -2.59598600 & -1.73731100 & 0.11646500 \\ \mathrm{C} & -3.97526300 & -1.09244300 & 0.03137500 \\ \mathrm{H} & -4.05211500 & -0.36047200 & 0.84679500 \\ \mathrm{H} & -4.01874100 & -0.50833000 & -0.89771800 \\ \mathrm{C} & -5.12167900 & -2.10120300 & 0.09649500 \\ \mathrm{H} & -6.08573900 & -1.58599600 & 0.03594200 \\ \mathrm{H} & -5.05932100 & -2.81874100 & -0.72616300 \\ \mathrm{H} & -5.08959800 & -2.67032600 & 1.02938900 \\ \mathrm{H} & -1.13234100 & 1.65453000 & -0.35992900 \\ \mathrm{H} & -1.32452800 & 2.03174700 & -1.28820100 \\ \mathrm{O} & -1.55664700 & -0.87575700 & 0.04282600 \\ \mathrm{H} & -1.76882700 & 0.11538900 & -0.09337900 \\ \mathrm{O} & 2.41286200 & -0.45364800 & -1.28482600 \\ \mathrm{H} & 2.51909500 & -0.00239900 & 0.87778500 \\ \mathrm{H} & 3.03068900 & -0.51987200 & -0.16369400 \\ \mathrm{H} & 4.36663600 & -1.23224200 & -0.11456900 \\ \mathrm{H} & 4.98549800 & -0.80238800 & -0.91118700 \\ \mathrm{H} & 4.17451600 & -2.26759000 & -0.42331400 \\ \mathrm{H} & -1.0769300 & -1.18203400 & 1.23843600 \\ \mathrm{H} & -1.63047400 & 2.02384300 \\ \mathrm{H} & -1.72851400 & 1.18682400 \\ \mathrm{H} & -0.15153500 & 1.53593900\end{array}$

C"

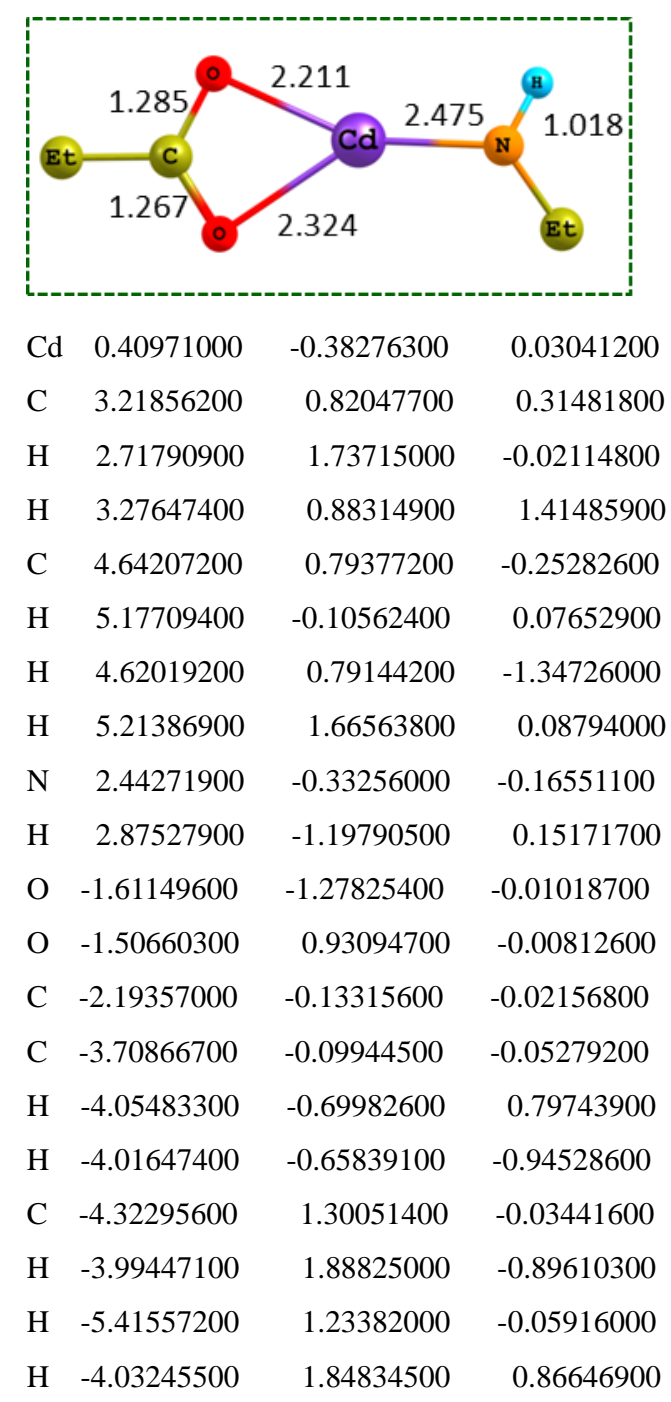

A/C-TS1

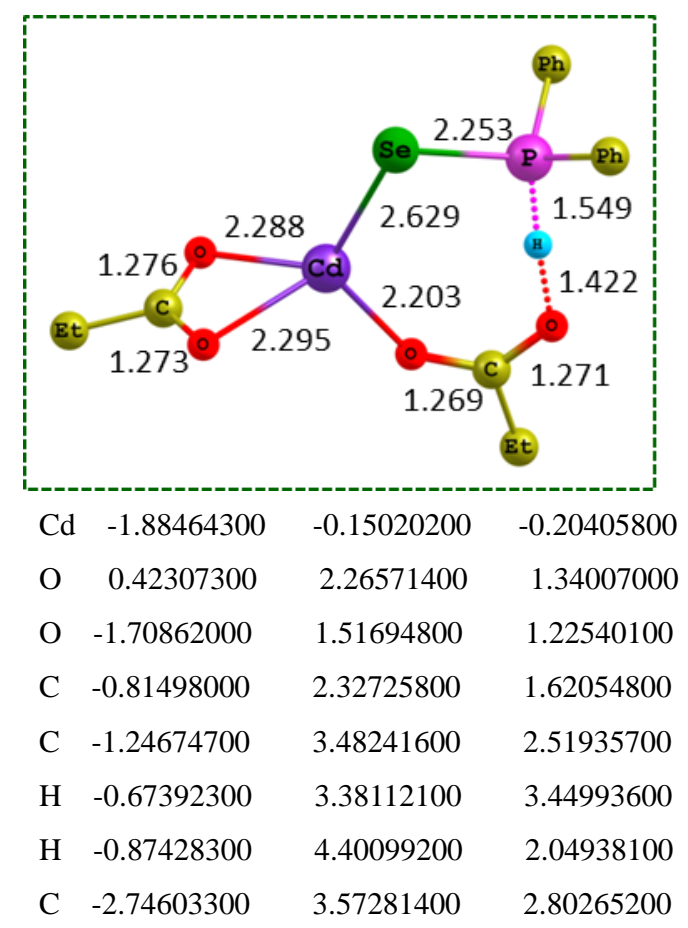




\begin{tabular}{|c|c|c|c|}
\hline & -2.95598800 & 4.42580700 & 3.45691800 \\
\hline & -3.31792900 & 3.70052800 & 1.87904900 \\
\hline & -3.11225000 & 2.66579000 & 3.29152800 \\
\hline & -3.95075500 & -0.01444800 & .19361800 \\
\hline & -3.64131800 & -1.45279400 & 0.46735400 \\
\hline & -4.39512800 & -0.91199400 & -0.40805800 \\
\hline & -5.84476200 & -1.35997700 & -0.49115800 \\
\hline & -6.29390600 & -1.14334800 & 0.48636500 \\
\hline & -5.83443000 & -2.45371400 & -0.56993500 \\
\hline & -6.65653700 & -0.72735500 & -1.62216400 \\
\hline & -6.22312200 & -0.96160700 & -2.59889300 \\
\hline & -7.68658300 & -1.09976600 & -1.60496000 \\
\hline & -6.68152400 & 0.36190100 & -1.52871700 \\
\hline & 1.74430900 & 0.00228600 & -0.05329400 \\
\hline & 2.53601400 & -1.1 & 1000 \\
\hline & 2.14335900 & -2.53557200 & 1.16044200 \\
\hline & 3.53269100 & -0.71235400 & 1.95694200 \\
\hline & 2.74578400 & -3.39165800 & 2.08777500 \\
\hline & 1.37160800 & -2.90811300 & 0.49393700 \\
\hline & 4.13007000 & -1.57120700 & 2.87957700 \\
\hline & 3.84367900 & 0.32803200 & 1.91329200 \\
\hline & 3.73752500 & -2.91284600 & 2.94630600 \\
\hline & 2.43666800 & -4.43171600 & 2.13597900 \\
\hline & 4.89890800 & -1.19254300 & 3.54674900 \\
\hline & 4.20286400 & -3.57954800 & 3.66653900 \\
\hline & 3.08455500 & 0.74447500 & -1.03975100 \\
\hline & 3.07722500 & 2.13550500 & -1.23341100 \\
\hline & 4.09183300 & -0.03727000 & -1.62808500 \\
\hline & 4.07343400 & 2.73639000 & -2.00774100 \\
\hline & 2.30276300 & 2.74547400 & -0.77668100 \\
\hline & 5.08326900 & 0.56906200 & -2.39977200 \\
\hline & 4.10422600 & -1.11340300 & -1.48069900 \\
\hline & 5.07429100 & 1.95544500 & -2.59127000 \\
\hline & 4.06662100 & 3.81283800 & -2.15156700 \\
\hline & 5.86096800 & -0.03897400 & -2.85261400 \\
\hline & 5.84739100 & 2.42446600 & -3.19307200 \\
\hline & 0.27614000 & -1.02451800 & -1.41982000 \\
\hline & 1.01348900 & 1.14191200 & 0.69888700 \\
\hline
\end{tabular}

A/C-IM1

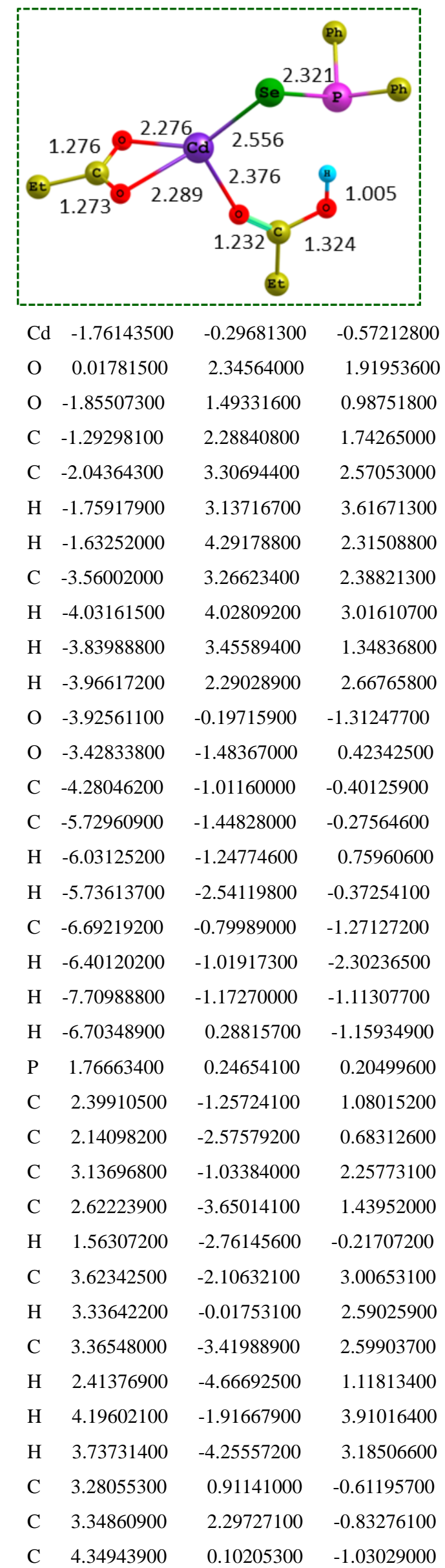




$\begin{array}{lrrr}\mathrm{C} & 4.46027300 & 2.86310900 & -1.46150300 \\ \mathrm{H} & 2.52993700 & 2.93684800 & -0.51295200 \\ \mathrm{C} & 5.46181500 & 0.66936900 & -1.65541000 \\ \mathrm{H} & 4.31482800 & -0.97032700 & -0.86436100 \\ \mathrm{C} & 5.51948400 & 2.04966500 & -1.87364200 \\ \mathrm{H} & 4.49955600 & 3.93631500 & -1.62543200 \\ \mathrm{H} & 6.28239600 & 0.03268300 & -1.97426000 \\ \mathrm{H} & 6.38606500 & 2.48839800 & -2.36011500 \\ \mathrm{Se} & 0.55770900 & -0.51193500 & -1.62575200 \\ \mathrm{H} & 0.51207500 & 1.66452300 & 1.37060200\end{array}$

C/E-IM1

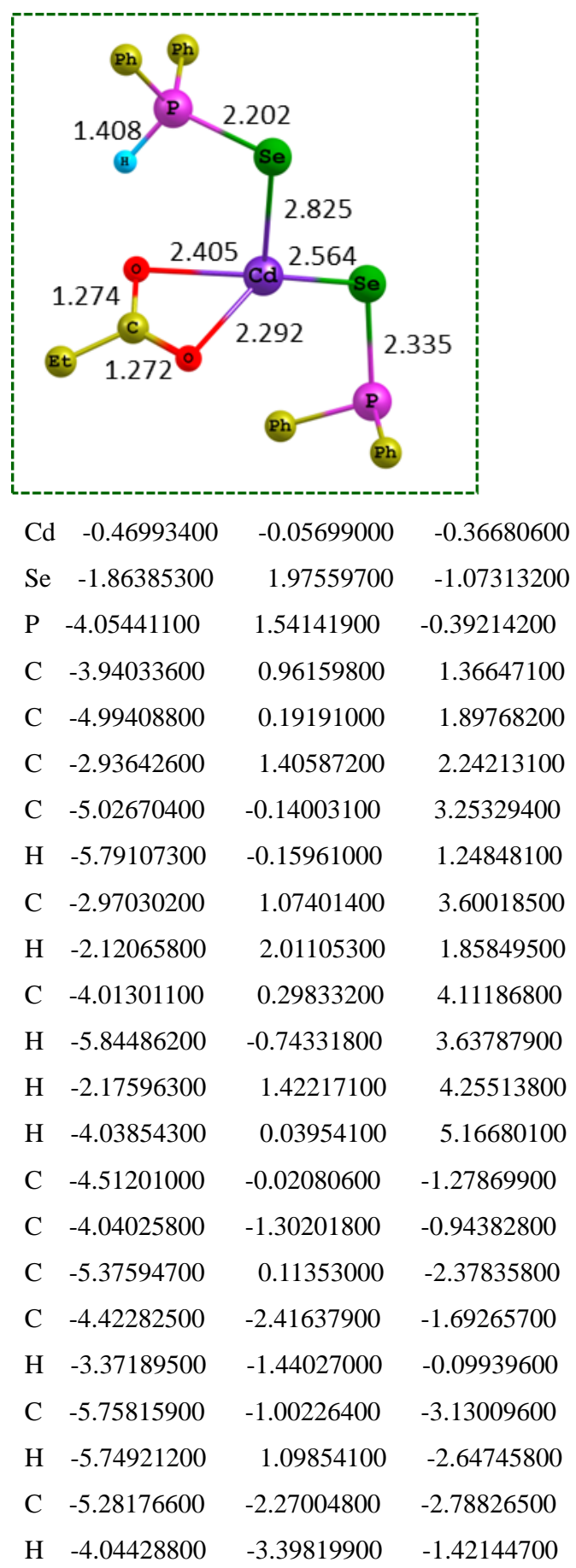

\begin{tabular}{|c|c|c|c|}
\hline & -6.42690500 & -0.87957800 & -3.97778500 \\
\hline & -5.57698200 & -3.13928600 & -3.36973600 \\
\hline & 0.50393000 & -0.87570700 & 1.67437400 \\
\hline & -1.00849100 & -2.07422400 & 0.57807900 \\
\hline & -0.24018800 & -1.90570700 & 1.57715700 \\
\hline & -0.20098800 & -2.94114500 & 2.69144600 \\
\hline & 0.84386700 & -3.26167600 & 2.79323100 \\
\hline & -0.43312200 & -2.40667700 & 3.62080600 \\
\hline & -1.12855700 & -4.14158000 & 2.49986500 \\
\hline & -1.03453800 & -4.83373800 & 3.34375000 \\
\hline & -2.17252800 & -3.82363500 & 2.43158800 \\
\hline & -0.88961500 & -4.68423200 & 1.58069500 \\
\hline & 1.94230200 & -0.29008800 & -1.81760100 \\
\hline F & 3.24541500 & -0.10803000 & -0.05236200 \\
\hline & 2.59566100 & -0.63986200 & 1.07799500 \\
\hline & 3.69790500 & 1.60909800 & 0.38012000 \\
\hline & 4.55373200 & 1.83436900 & 1.47245800 \\
\hline & 3.17232000 & 2.69308600 & -0.33274200 \\
\hline & 4.87760700 & 3.13929100 & 1.84312800 \\
\hline & 4.96916900 & 0.99914300 & 2.02995600 \\
\hline & 3.50353900 & 3.99856900 & 0.04250900 \\
\hline & 2.50802700 & 2.51546700 & -1.17304900 \\
\hline & 4.35280600 & 4.22213500 & 1.12795300 \\
\hline & 5.53690000 & 3.31086900 & 2.68882500 \\
\hline & 3.09125200 & 4.83593700 & -0.51214400 \\
\hline & 4.60580500 & 5.23731400 & 1.41961700 \\
\hline & 4.78257200 & -1.06904600 & -0.17937700 \\
\hline & 4.89357900 & -2.28618600 & 0.51004200 \\
\hline & 5.83249400 & -0.63207200 & -1.00244500 \\
\hline & 6.05410800 & -3.05399400 & 0.38579200 \\
\hline & 4.08061000 & -2.63573400 & 1.14045800 \\
\hline & 6.98873800 & -1.40312100 & -1.12191000 \\
\hline & 5.75004700 & 0.30633000 & -1.54337100 \\
\hline & 7.10042400 & -2.61354300 & -0.42892500 \\
\hline & 6.13833000 & -3.99349200 & 0.92371400 \\
\hline & 7.80050000 & -1.06134500 & -1.75698200 \\
\hline & 8.00148900 & -3.21208400 & -0.52533400 \\
\hline
\end{tabular}

C-i

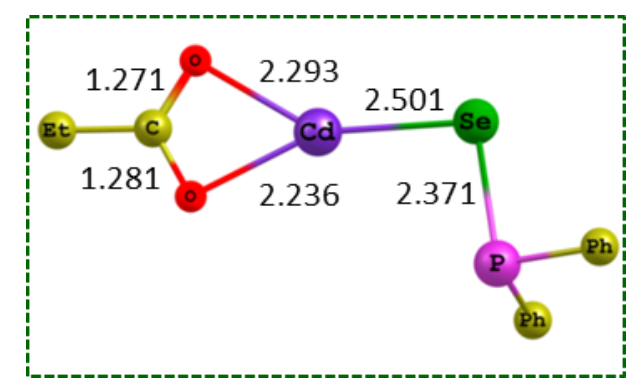




\begin{tabular}{|c|c|c|c|}
\hline $\mathrm{d}$ & 0 & 0 & 0 \\
\hline $\mathrm{O}$ & 3.53309900 & 0.37969700 & 1.30287600 \\
\hline & 4.30665100 & -0.48756800 & -0.58033900 \\
\hline & 4.51668700 & 05146300 & 0.55121600 \\
\hline & 5.92 & 00 & 3500 \\
\hline $\mathrm{H}$ & 6.02669400 & -0.21244000 & 1.99916100 \\
\hline & 5.96961500 & 38453700 & 5400 \\
\hline 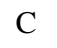 & 7.03775000 & 3900 & 2800 \\
\hline $\mathrm{H}$ & 6.94845300 & 0.47980700 & 7500 \\
\hline 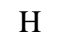 & 8.01589100 & 15708000 & 2700 \\
\hline $\mathrm{H}$ & 7.00 & 3300 & -0.1 \\
\hline$P$ & -1.43828800 & -0.07812900 & 65600 \\
\hline $\mathrm{C}$ & -2.98800500 & -1.05 & 5100 \\
\hline $\mathrm{C}$ & -3.11 & -2.2 & 0 \\
\hline$C$ & -4.02967400 & -0.65849800 & -0.35472700 \\
\hline$C$ & -4.25219500 & -3.05430200 & 1.07067600 \\
\hline$H$ & -2.32 & 0 & 0 \\
\hline $\mathrm{C}$ & -5.16870700 & -1.45487700 & -0.49555400 \\
\hline$\Pi$ & -3.95 & 0.21 & 0 \\
\hline $\mathrm{C}$ & -5.28203800 & -2.65459700 & 0.21365100 \\
\hline $\mathrm{H}$ & -4.33511300 & -3.98283900 & 1.62857800 \\
\hline $\mathrm{H}$ & -5.96708700 & -1.13832900 & -1.16118100 \\
\hline $\mathrm{H}$ & -6.16912600 & -3.27174400 & 0.10219500 \\
\hline $\mathrm{C}$ & -1.97176900 & 100 & 1900 \\
\hline$C$ & -1.8358 & 2.2123 & $-0.9^{\prime}$ \\
\hline $\mathrm{C}$ & -2.52520000 & 2.40584500 & 1.33271600 \\
\hline $\mathrm{C}$ & -2.25687500 & 3.52621700 & -1.20574000 \\
\hline 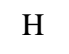 & -1.394 & 1.6 & -1.7 \\
\hline $\mathrm{C}$ & -2.95562200 & 3.7137 & 1.09957000 \\
\hline $\mathrm{H}$ & -2.61 & 1. & 0 \\
\hline $\mathrm{C}$ & -2.82053100 & 4.27890000 & -0.17237700 \\
\hline $\mathrm{H}$ & -2.14347300 & 3.95936500 & -2.19594200 \\
\hline 11 & -3.38436500 & 2200 & 1.91272900 \\
\hline $\mathrm{H}$ & -3.14522900 & 5.29958900 & -0.35376100 \\
\hline $\mathrm{Se}$ & -0.22737400 & -0.86362400 & -1.1008640 \\
\hline
\end{tabular}

C

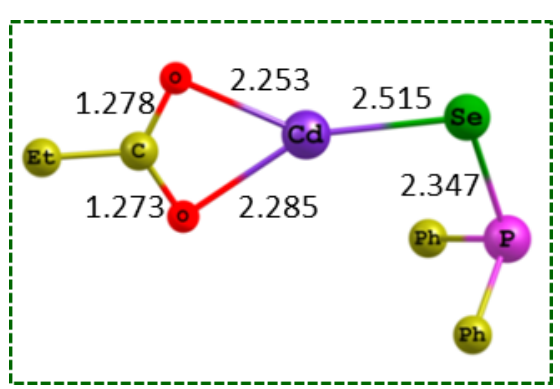

$\begin{array}{llll}\mathrm{Cd} & 1.28084400 & 0.00127200 & -1.00375300\end{array}$

Se $\quad-0.85256400 \quad 0.29999000 \quad-2.30076100$

$\begin{array}{lrrr}\mathrm{P} & -2.46655800 & 0.40479600 & -0.59992400 \\ \mathrm{C} & -1.71339700 & 1.58599700 & 0.61250800 \\ \mathrm{C} & -2.13959400 & 2.92271900 & 0.53884900 \\ \mathrm{C} & -0.74074800 & 1.23883800 & 1.56726600 \\ \mathrm{C} & -1.59818500 & 3.89301500 & 1.38722900 \\ \mathrm{H} & -2.89498000 & 3.20478500 & -0.19018500 \\ \mathrm{C} & -0.20045600 & 2.20804800 & 2.41571400 \\ \mathrm{H} & -0.41088300 & 0.20830900 & 1.66528400 \\ \mathrm{C} & -0.62646600 & 3.53789700 & 2.32647800 \\ \mathrm{H} & -1.93779700 & 4.92245100 & 1.31547300 \\ \mathrm{H} & 0.55142000 & 1.92161300 & 3.14564000 \\ \mathrm{H} & -0.20572900 & 4.29022800 & 2.98759600 \\ \mathrm{C} & -2.35015800 & -1.20693300 & 0.30868300 \\ \mathrm{C} & -2.92458800 & -1.28117200 & 1.59314500 \\ \mathrm{C} & -1.88194000 & -2.39081600 & -0.28222700 \\ \mathrm{C} & -2.99948000 & -2.49751500 & 2.27332500 \\ \mathrm{H} & -3.30877600 & -0.38403300 & 2.07130400 \\ \mathrm{H} & -1.20800 \\ \mathrm{C} & -1.96157800 & -3.60983500 & 0.39939000 \\ \mathrm{H} & -1.45422300 & -2.36155700 & -1.27966600 \\ \mathrm{C} & -2.51569200 & -3.66801000 & 1.67960700 \\ \mathrm{H} & -3.43600500 & -2.52968300 & 3.26781400 \\ \mathrm{H} & -1.58785900 & -4.51326300 & -0.07482000 \\ \mathrm{H} & -2.57464200 & -4.61451200 & 2.20918200 \\ \mathrm{H} & 3.53333700 & 0.01822700 & -0.93858500 \\ \mathrm{H} & 2.43002200 & -0.58516000 & 0.88220400 \\ \mathrm{H} & 3.53011400 & -0.37654200 & 0.27722100 \\ \mathrm{H} & -.45924300 & -0.59479300 & 0.97354600 \\ \mathrm{H} & -1.33962600 & 0.37844300 \\ \mathrm{H} & -1.95594800 & 2.54452000\end{array}$

C/E-TS1

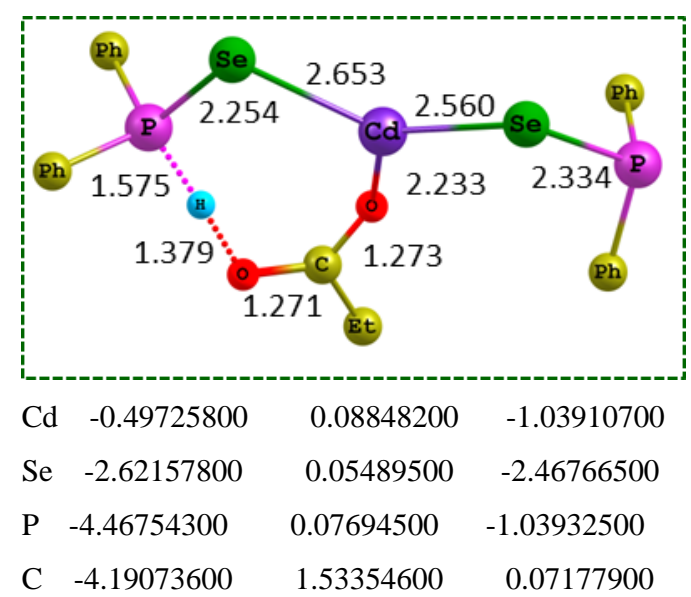




\begin{tabular}{|c|c|c|c|}
\hline$c$ & -3.23958000 & 1.58193100 & 1.106148 \\
\hline$C$ & -4.98567900 & 2.66613900 & -0.16940800 \\
\hline$C$ & -3.08850300 & 2.73847900 & 1.87372500 \\
\hline $\mathrm{H}$ & -2.61248800 & 0.71952500 & 1.31358600 \\
\hline $\mathrm{C}$ & -4.83313800 & 3.82501200 & 0.59913600 \\
\hline $\mathrm{H}$ & -5.72653600 & 2.64012500 & -0.96453500 \\
\hline $\mathrm{C}$ & -3.88341400 & 3.86331200 & 1.62240000 \\
\hline $\mathrm{H}$ & -2.34717300 & 2.76238300 & 2.66834800 \\
\hline $\mathrm{H}$ & -5.45604400 & 4.69237300 & 0300 \\
\hline $\mathrm{H}$ & -3.76274700 & 4.76150900 & 2.22191000 \\
\hline $\mathrm{C}$ & -4.24634500 & -1.35397800 & 0.11919000 \\
\hline $\mathrm{C}$ & -3.63268000 & $-2.54 \mathrm{C}$ & 5400 \\
\hline $\mathrm{C}$ & -4.88691100 & -1.32814600 & 1.37302600 \\
\hline $\mathrm{C}$ & -3.64377800 & -3.67896400 & 0.53498600 \\
\hline $\mathrm{H}$ & -3.14063400 & -2.59532800 & -1.25624100 \\
\hline $\mathrm{C}$ & -4.89650500 & -2.45625300 & 2.19653100 \\
\hline $\mathrm{H}$ & -5.37875900 & -0.42160800 & 1.71402600 \\
\hline $\mathrm{C}$ & -4.27423700 & -3.63803500 & 1.78179700 \\
\hline $\mathrm{H}$ & -3.15993600 & -4.59225400 & 0.19846300 \\
\hline $\mathrm{H}$ & -5.39246900 & -2.41038100 & 54000 \\
\hline $\mathrm{H}$ & -4.28518100 & -4.51686000 & 2.42052900 \\
\hline $\mathrm{O}$ & 1.28293900 & -1.63943300 & 1.72823300 \\
\hline $\mathrm{O}$ & -0.55017100 & -0.47 & 5000 \\
\hline $\mathrm{C}$ & 0.07501100 & -1.27575200 & 1.88489600 \\
\hline $\mathrm{C}$ & -0.70226100 & -1.81899800 & 9300 \\
\hline $\mathrm{H}$ & -1.03935800 & -0.94619000 & 3.65016900 \\
\hline $\mathrm{H}$ & -1.61706300 & -2.27039600 & 2.67461600 \\
\hline $\mathrm{C}$ & 0.05837800 & -2.80053700 & 3.96765700 \\
\hline $\mathrm{H}$ & -0.57872300 & -3.12791200 & 4.79611000 \\
\hline $\mathrm{H}$ & 0.37017200 & -3.68633800 & 3.40650300 \\
\hline $\mathrm{H}$ & 0.95912800 & -2.34345300 & 4.38741300 \\
\hline $\mathrm{Se}$ & 1.96747900 & 0.69642900 & -1.80831000 \\
\hline$P$ & 3.05750700 & 0.01069100 & 0.04123800 \\
\hline $\mathrm{H}$ & 2.08452200 & -0.85148300 & 0.92987300 \\
\hline $\mathrm{C}$ & 3.76141300 & 1.44083100 & 0.94944400 \\
\hline $\mathrm{C}$ & 4.61328800 & 1.18122000 & 2. \\
\hline $\mathrm{C}$ & 3.42335000 & 2.76393000 & 0.63777600 \\
\hline $\mathrm{C}$ & 5.12026800 & 2.23553500 & 2.79857300 \\
\hline $\mathrm{H}$ & 4.88387800 & 0.15987100 & 2.29241600 \\
\hline $\mathrm{C}$ & 3.93508600 & 3.81695100 & 1.40269800 \\
\hline $\mathrm{H}$ & 2.76656400 & 2.96792000 & -0.20225000 \\
\hline $\mathrm{C}$ & 4.78214200 & 3.55596200 & 2.48176600 \\
\hline $\mathrm{H}$ & 5.77810700 & 2.02585000 & 3.63704300 \\
\hline $\mathrm{H}$ & 3.67030200 & 4.83979100 & 1.15084000 \\
\hline $\mathrm{H}$ & 5.17804200 & 4.37546100 & 3.07464100 \\
\hline $\mathrm{C}$ & 4.47548400 & -1.03207800 & -0.43974500 \\
\hline
\end{tabular}

$\begin{array}{lrrr}\mathrm{C} & 4.38848700 & -2.41748400 & -0.22725700 \\ \mathrm{C} & 5.62234100 & -0.49109400 & -1.04333600 \\ \mathrm{C} & 5.44211200 & -3.25102400 & -0.61326400 \\ \mathrm{H} & 3.50572800 & -2.84336700 & 0.24175000 \\ \mathrm{C} & 6.67086400 & -1.32777400 & -1.42569100 \\ \mathrm{H} & 5.69645400 & 0.57946700 & -1.21157500 \\ \mathrm{C} & 6.58134800 & -2.70825200 & -1.21254500 \\ \mathrm{H} & 5.37109900 & -4.32144600 & -0.44384500 \\ \mathrm{H} & 7.55566000 & -0.90435400 & -1.89206700 \\ \mathrm{H} & 7.39879700 & -3.35743200 & -1.51281600\end{array}$

C/E-IM2

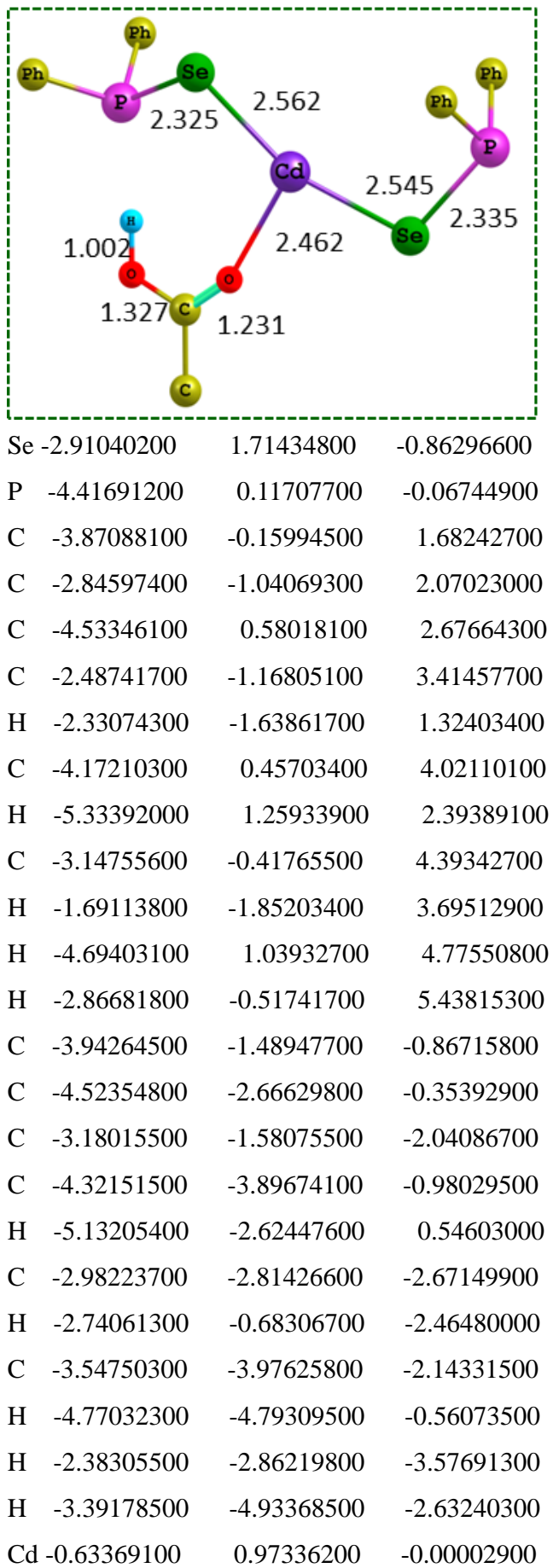




\begin{tabular}{|c|c|c|c|}
\hline $\mathrm{Se}$ & 1.13036500 & -0.36191200 & 1.29271400 \\
\hline $\mathrm{H}$ & 2.71661200 & 1.83710600 & -1.33201400 \\
\hline $\mathrm{C}$ & 1.44977500 & 4.61369600 & -2.47276300 \\
\hline $\mathrm{H}$ & 1.75840300 & 4.42882300 & -3.50950100 \\
\hline $\mathrm{H}$ & 2.24022000 & 5.24278200 & -2.04389100 \\
\hline $\mathrm{O}$ & 2.69363200 & 2.69837400 & -1.84381300 \\
\hline $\mathrm{C}$ & 1.50672700 & 3.28514000 & -1.75260500 \\
\hline $\mathrm{O}$ & 0.55089000 & 2.80170600 & -1.14674000 \\
\hline $\mathrm{C}$ & 0.08704600 & 5.30150800 & -2.40652400 \\
\hline $\mathrm{H}$ & 0.12154900 & 6.25146800 & -2.94831200 \\
\hline $\mathrm{H}$ & -0.69440100 & 4.67970300 & -2.85169900 \\
\hline $\mathrm{H}$ & -0.20410800 & 5.50611400 & -1.37267500 \\
\hline $\mathrm{P}$ & 2.93815600 & -0.04889200 & -0.13587500 \\
\hline $\mathrm{C}$ & 3.09545700 & -1.64749900 & -1.04389700 \\
\hline $\mathrm{C}$ & 3.16418600 & -2.89606100 & -0.40191800 \\
\hline $\mathrm{C}$ & 3.12115900 & -1.60227000 & -2.44637100 \\
\hline $\mathrm{C}$ & 3.26267200 & -4.06957800 & -1.14861300 \\
\hline $\mathrm{H}$ & 3.13247000 & -2.94873400 & 0.68240000 \\
\hline $\mathrm{C}$ & 3.22040800 & -2.78001700 & -3.19614000 \\
\hline $\mathrm{H}$ & 3.06551700 & -0.64442500 & -2.95771500 \\
\hline $\mathrm{C}$ & 3.29087400 & -4.01421200 & -2.54796800 \\
\hline $\mathrm{H}$ & 3.31321300 & -5.02878600 & -0.64090900 \\
\hline $\mathrm{H}$ & 3.24061000 & -2.72954500 & -4.28114800 \\
\hline $\mathrm{H}$ & 3.36459500 & -4.93049200 & -3.12690800 \\
\hline $\mathrm{C}$ & 4.37705600 & -0.08889100 & 1.02575200 \\
\hline $\mathrm{C}$ & 5.59692200 & -0.65619400 & 0.61512500 \\
\hline $\mathrm{C}$ & 4.32473200 & 0.57350300 & 2.26392500 \\
\hline $\mathrm{C}$ & 6.73002200 & -0.57077700 & 1.42806100 \\
\hline $\mathrm{H}$ & 5.66555900 & -1.17303800 & -0.33717500 \\
\hline $\mathrm{C}$ & 5.45931700 & 0.65547400 & 3.07473400 \\
\hline $\mathrm{H}$ & 3.39038800 & 1.01018200 & 2.60281700 \\
\hline $\mathrm{C}$ & 6.66583700 & 0.08396500 & 2.66106400 \\
\hline $\mathrm{H}$ & 7.66151900 & -1.02136400 & 1.09660400 \\
\hline $\mathrm{H}$ & 5.39625700 & 1.16306000 & 4.03333900 \\
\hline $\mathrm{H}$ & 7.54650000 & 0.14625900 & 3.29391100 \\
\hline
\end{tabular}

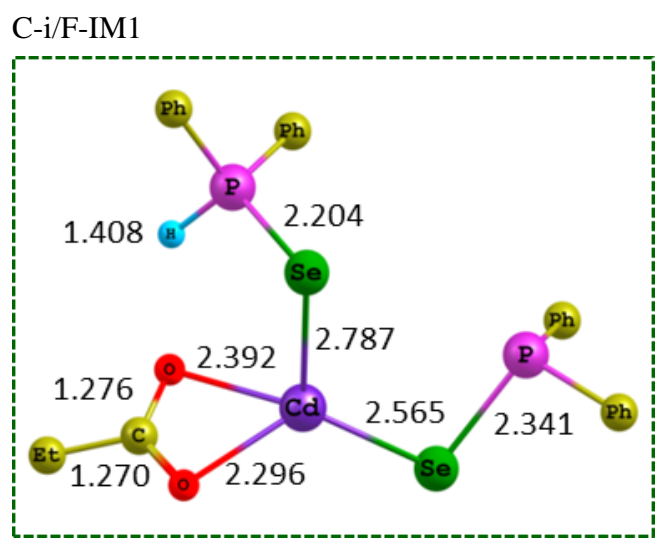

\begin{tabular}{|c|c|c|c|}
\hline & 4000 & 1.85472700 & \\
\hline & & 1.72007400 & -0.62903900 \\
\hline$P$ & -2.68517500 & -0.30164100 & 0.48231300 \\
\hline C & -3.41826500 & -1.39303000 & -0.83422600 \\
\hline $\mathrm{C}$ & -4.11246200 & -2.54519100 & -0.41322800 \\
\hline & -3.19366000 & -1.2 & 61300 \\
\hline & -4.57845200 & -3.48039100 & -1.33894600 \\
\hline $\mathrm{H}$ & -4.30111400 & -2.70868400 & 0.64511700 \\
\hline C & -3.65733300 & -2.15647300 & -3.13518700 \\
\hline H & -2.66594500 & -0.33236200 & -2.55272300 \\
\hline C & -4.35157400 & -3.29000500 & 19400 \\
\hline $\mathrm{H}$ & -5.12247600 & -4.35498200 & -0.99179100 \\
\hline $\mathrm{H}$ & -3.47942100 & -1.99406500 & -4.19504800 \\
\hline $\mathrm{H}$ & -4.71655800 & -4.01 & -3 \\
\hline $\mathrm{C}$ & 21200 & 0.12675400 & 2500 \\
\hline$C$ & -5.45489700 & 0.39327200 & 0.88405800 \\
\hline$c$ & -4.06506700 & 0.20906100 & 2.85388800 \\
\hline C & -6.5 & 00 & 000 \\
\hline $\mathrm{H}$ & -5.57784100 & 0.33795300 & -0.19363700 \\
\hline $\mathrm{C}$ & -5.15729800 & 0.54744100 & 3.65943500 \\
\hline $\mathrm{H}$ & -3.09979500 & 0.00620300 & 3.31121000 \\
\hline C & -6.39982500 & 0.80751300 & 3.07696500 \\
\hline $\mathrm{H}$ & -7.50964100 & 0.93364700 & 1.22848200 \\
\hline $\mathrm{H}$ & -5.03620600 & 0.60598800 & 4.73771600 \\
\hline $\mathrm{H}$ & -7.25036100 & 1.07012200 & 3.70001400 \\
\hline $\mathrm{O}$ & 2.12744100 & 1.86572700 & -1.75304800 \\
\hline $\mathrm{O}$ & 1.51431300 & 3.72171900 & -0.69884900 \\
\hline C & 2.27306300 & 3.11079500 & -1.51343800 \\
\hline & 3.37885300 & 3.86490900 & -2.2 \\
\hline $\mathrm{H}$ & 4.31296200 & 3.31896600 & -2.05496000 \\
\hline $\mathrm{H}$ & 3.18235200 & 3.75024500 & -3.31168600 \\
\hline$c$ & 3.51707300 & 5.33972000 & -1.85848300 \\
\hline $\mathrm{H}$ & 4.32789300 & 5.80587200 & -2.42896500 \\
\hline $\mathrm{H}$ & 2.59262800 & 5.88663000 & -2.06312600 \\
\hline $\mathrm{H}$ & 3.73478200 & 5.45524900 & -0.79276900 \\
\hline $\mathrm{Se}$ & 1.54094100 & 32558600 & 1.66635900 \\
\hline & 2.79217800 & -0.76441300 & 0.21593200 \\
\hline $\mathrm{H}$ & 3.16679000 & 0.09394800 & -0.83546500 \\
\hline$C$ & 1.98004000 & -2.20377600 & -0.55965400 \\
\hline 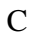 & 2.72357900 & -3.01629700 & -1.43418700 \\
\hline C & 0.62486600 & -2.47319100 & -0.33196500 \\
\hline $\mathrm{C}$ & 2.10692900 & -4.09101300 & -2.07396100 \\
\hline H & 3.77643400 & -2.81644900 & -1.61432400 \\
\hline$c$ & 0.01298000 & -3.55087800 & -0.98015000 \\
\hline $\mathrm{H}$ & 0.04706500 & -1.84715400 & 0.34227800 \\
\hline $\mathrm{C}$ & 0.75133400 & -4.35763200 & -1.84778200 \\
\hline
\end{tabular}




$\begin{array}{rrrr}\mathrm{H} & 2.68197900 & -4.71678600 & -2.75011800 \\ \mathrm{H} & -1.04154800 & -3.74537100 & -0.81156900 \\ \mathrm{H} & 0.27207600 & -5.19162000 & -2.35224900 \\ \mathrm{C} & 4.36977400 & -1.32331800 & 0.92157800 \\ \mathrm{C} & 5.54318900 & -0.60625200 & 0.64262700 \\ \mathrm{C} & 4.41251100 & -2.42866300 & 1.78584400 \\ \mathrm{C} & 6.75394200 & -1.00129400 & 1.21663100 \\ \mathrm{H} & 5.51485400 & 0.25657300 & -0.01716500 \\ \mathrm{C} & 5.62468500 & -2.81766700 & 2.35612200 \\ \mathrm{H} & 3.50632700 & -2.98467500 & 2.00859700 \\ \mathrm{C} & 6.79505000 & -2.10525400 & 2.07237500 \\ \mathrm{H} & 7.66093800 & -0.44673600 & 0.99546900 \\ \mathrm{H} & 5.65568700 & -3.67449300 & 3.02243500 \\ \mathrm{H} & 7.73694700 & -2.41036000 & 2.51872300\end{array}$

C-i/E-ii-TS1

\begin{tabular}{|c|c|c|c|}
\hline & Ph 2.25 & 640 & $\mathrm{ph}$ \\
\hline $\mathrm{Cd}$ & -0.31495700 & .70156400 & -0.99297000 \\
\hline $\mathrm{Se}$ & -2.70049300 & 1.59233700 & -1.04460000 \\
\hline$P$ & -3.55847400 & -0.05141500 & 0.40334600 \\
\hline $\mathrm{C}$ & -4.73496700 & -1.02520700 & -0.65741500 \\
\hline $\mathrm{C}$ & -5.50128500 & -2.00620200 & 0.00178600 \\
\hline $\mathrm{C}$ & -4.83889500 & -0.92117000 & -2.05113300 \\
\hline $\mathrm{C}$ & -6.36326400 & -2.84029400 & -0.71104000 \\
\hline $\mathrm{H}$ & -5.42793100 & -2.11590800 & 1.08154000 \\
\hline $\mathrm{C}$ & -5.69756900 & -1.76368800 & -2.76737000 \\
\hline $\mathrm{H}$ & -4.25142500 & -0.17496900 & -2.57724100 \\
\hline $\mathrm{C}$ & -6.46416400 & -2.72218900 & -2.10209300 \\
\hline $\mathrm{H}$ & -6.95195200 & -3.58487400 & -0.18183400 \\
\hline $\mathrm{H}$ & -5.76720600 & -1.66357700 & -3.84742600 \\
\hline $\mathrm{H}$ & -7.13159100 & -3.37393700 & -2.65907800 \\
\hline $\mathrm{C}$ & -4.74768400 & 0.97263700 & 1.38939200 \\
\hline $\mathrm{C}$ & -5.99722400 & 1.40675100 & 0.91597800 \\
\hline $\mathrm{C}$ & -4.35539500 & 1.33443200 & 2.68909700 \\
\hline $\mathrm{C}$ & -6.83166100 & 2.18194700 & 1.72371000 \\
\hline $\mathrm{H}$ & -6.31998400 & 1.13516800 & -0.08463200 \\
\hline $\mathrm{C}$ & -5.18829900 & 2.11390800 & 3.49683200 \\
\hline $\mathrm{H}$ & -3.39289700 & 1.00193600 & 3.07027700 \\
\hline
\end{tabular}

\begin{tabular}{|c|c|c|c|}
\hline C & -6.42934000 & 2.53895400 & 3.0 \\
\hline $\mathrm{H}$ & -7.79569100 & 250967200 & 134383300 \\
\hline H & -4.86979300 & 2.38366000 & 4.50010600 \\
\hline $\mathrm{H}$ & -7.08080900 & 3.14168900 & .64215700 \\
\hline C & 3.28663200 & 1.51907400 & -1.82930100 \\
\hline $\mathrm{O}$ & 1.09438300 & 1.88067100 & 6700 \\
\hline C & 2.32709000 & 2.17493700 & -2.34046400 \\
\hline $\mathrm{C}$ & 2.65149200 & 3.42347200 & -3.15366000 \\
\hline $\mathrm{H}$ & 2.19699000 & 3.27732800 & -4.14148900 \\
\hline $\mathrm{H}$ & 2.08864800 & 4.24738000 & -2.69774200 \\
\hline C & 4.13607500 & 3.76758600 & -3.27366300 \\
\hline $\mathrm{H}$ & 4.26618100 & 4.67377200 & -3.87482900 \\
\hline $\mathrm{H}$ & 4.58427200 & 3.94166700 & -2.29110500 \\
\hline $\mathrm{H}$ & 4.69486600 & 2.95710300 & -3.75053700 \\
\hline $\mathrm{Se}$ & 0.81847700 & 1.30598200 & 0100 \\
\hline $\mathrm{P}$ & 2.85089600 & .17063500 & -0.66973500 \\
\hline $\mathrm{H}$ & 3.04035800 & 0.24578000 & -1.30213500 \\
\hline $\mathrm{C}$ & 3.08389600 & -2.50 & -1.90 \\
\hline $\mathrm{C}$ & 4.27116000 & -2.49761400 & -2.65925800 \\
\hline $\mathrm{C}$ & 2.11979500 & -3.49307800 & -2.14240800 \\
\hline $\mathrm{C}$ & 4.48982700 & 3471 & -3.630 \\
\hline $\mathrm{H}$ & 5.02381000 & -1.73184800 & -2.48969800 \\
\hline $\mathrm{C}$ & 3400 & -4.4 & -3.11738200 \\
\hline $\mathrm{H}$ & 1.19703300 & -3.49411800 & -1.57014400 \\
\hline $\mathrm{C}$ & 3.52663100 & -4.46347500 & -3.86075600 \\
\hline $\mathrm{H}$ & 5.40847400 & -3.46103200 & -4.21020800 \\
\hline $\mathrm{H}$ & 1.59221600 & -5.23364500 & -3.29428500 \\
\hline $\mathrm{H}$ & 3.69741500 & -5.22186000 & -4.61938800 \\
\hline $\mathrm{C}$ & 400 & 11615 & 0.5997 \\
\hline $\mathrm{C}$ & 4.81010000 & -0.28703400 & 1.09442900 \\
\hline - & 4.44516300 & -2.68835200 & 1.10887100 \\
\hline $\mathrm{C}$ & 5.78137400 & -0.43220500 & 2.08 \\
\hline $\mathrm{H}$ & 4.58167000 & 0.69969400 & 0.70119600 \\
\hline $\mathrm{C}$ & 2500 & -2.02 & 2.10088600 \\
\hline $\mathrm{H}$ & 3.93176700 & -3.56694600 & 0.72898400 \\
\hline $\mathrm{C}$ & 6.08418000 & -1.69974500 & 2.59252100 \\
\hline 1 & 6.30111200 & 0.44402800 & 2.46474000 \\
\hline $\mathrm{H}$ & 5.65111000 & -3.81303700 & 2.49111400 \\
\hline H & 6.83962600 & -1.81092500 & 3.36503900 \\
\hline
\end{tabular}




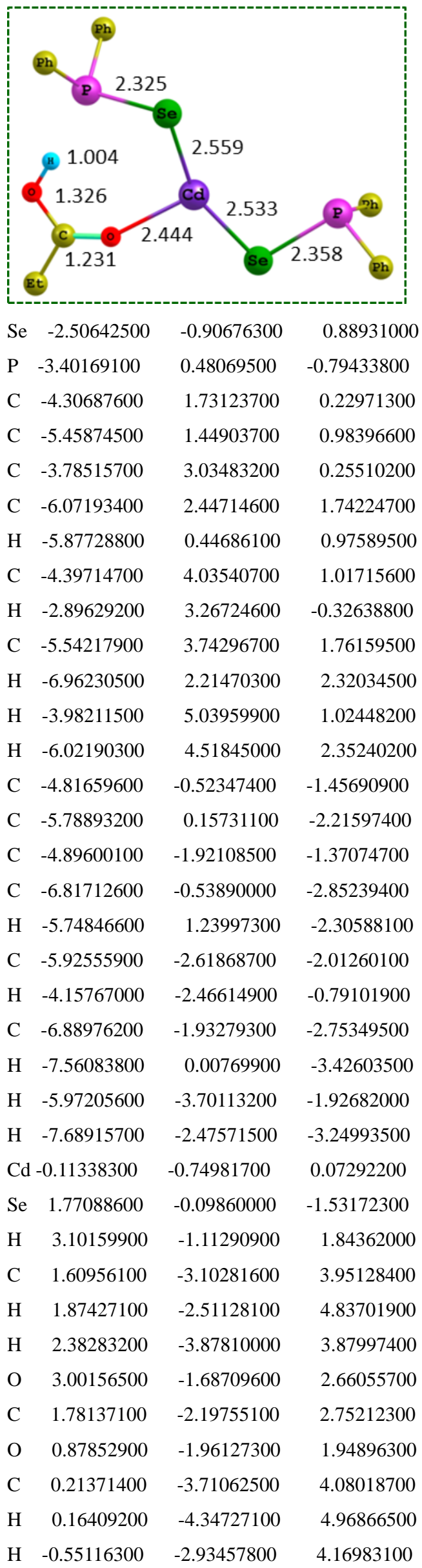

$\begin{array}{lrrr}\mathrm{H} & -0.03540200 & -4.32018100 & 3.20720500 \\ \mathrm{P} & 3.49800400 & 0.09819300 & 0.01296600 \\ \mathrm{C} & 3.73659800 & 1.91719400 & 0.20858500 \\ \mathrm{C} & 3.95479200 & 2.78514600 & -0.87514700 \\ \mathrm{C} & 3.67127600 & 2.45086500 & 1.50487800 \\ \mathrm{C} & 4.11088700 & 4.15437000 & -0.66246600 \\ \mathrm{H} & 3.99571200 & 2.38836500 & -1.88527100 \\ \mathrm{C} & 3.82824300 & 3.82509000 & 1.71863400 \\ \mathrm{H} & 3.49846900 & 1.79272600 & 2.35283000 \\ \mathrm{C} & 4.04823800 & 4.67752100 & 0.63539700 \\ \mathrm{H} & 4.27789500 & 4.81555400 & -1.50824700 \\ \mathrm{H} & 3.77652700 & 4.22423100 & 2.72767200 \\ \mathrm{H} & 4.16813600 & 5.74499700 & 0.79758100 \\ \mathrm{C} & 4.98385700 & -0.40482100 & -0.96710500 \\ \mathrm{C} & 6.23289100 & 0.17347700 & -0.67512300 \\ \mathrm{C} & 4.92914400 & -1.45821000 & -1.89465900 \\ \mathrm{C} & 7.39298400 & -0.28501700 & -1.30341900 \\ \mathrm{H} & 6.30355500 & 0.98893200 & 0.03847500 \\ \mathrm{C} & 6.09169900 & -1.91284200 & -2.52278400 \\ \mathrm{H} & 3.97421400 & -1.91267600 & -2.13952200 \\ \mathrm{C} & 7.32713800 & -1.32928700 & -2.23035900 \\ \mathrm{H} & 8.34730200 & 0.17900600 & -1.06993900 \\ \mathrm{H} & 6.02760500 & -2.72160100 & -3.24550000 \\ \mathrm{H} & 8.22943200 & -1.68253400 & -2.72130400\end{array}$

C-i/F-TS1

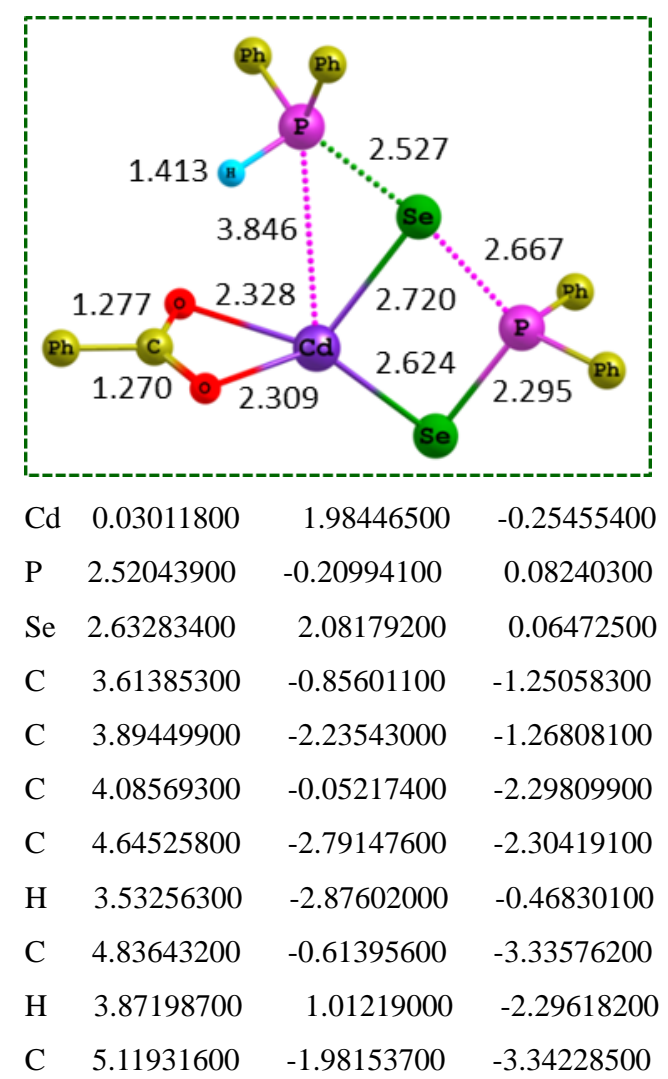




\begin{tabular}{|c|c|c|c|}
\hline $\mathrm{H}$ & 4.85962300 & -3.85663000 & -2.3 \\
\hline $\mathrm{H}$ & 5.20167200 & 0.02265000 & -4.13687900 \\
\hline $\mathrm{H}$ & 5.70363800 & -2.41522700 & -4.14889500 \\
\hline C & 3.33263100 & -0.77875400 & 1.63239600 \\
\hline $\mathrm{C}$ & 4.72985900 & -0.79702800 & 1.78154100 \\
\hline $\mathrm{C}$ & 2.52190400 & -1.16679800 & 2.71051700 \\
\hline $\mathrm{C}$ & 5.30307000 & -1.20153700 & 2.98792200 \\
\hline $\mathrm{H}$ & 5.36856800 & 800 & 0 \\
\hline $\mathrm{C}$ & 3.09953400 & -1.56839700 & 3.91848300 \\
\hline $\mathrm{H}$ & 1.44110500 & -1.15393000 & 500 \\
\hline $\mathrm{C}$ & 4.48935200 & -1.587 & $4.05 \mathrm{c}$ \\
\hline $\mathrm{H}$ & 6.38439700 & -1.21170000 & 3.09326700 \\
\hline $\mathrm{H}$ & 2.46255300 & -1.86809600 & 4.745 \\
\hline $\mathrm{H}$ & 4.93812900 & -1.90057500 & 4.99754900 \\
\hline $\mathrm{Se}$ & -0.08960000 & -0.72803100 & -0.09589800 \\
\hline $\mathrm{O}$ & -1.91519600 & 2.97167900 & 0.55969800 \\
\hline $\mathrm{O}$ & -1.38414000 & 3.24310300 & -1.57576800 \\
\hline $\mathrm{C}$ & -2.15375900 & 3.47068700 & -0.591 \\
\hline $\mathrm{C}$ & -3.37756000 & 4.35771000 & -0.75923600 \\
\hline $\mathrm{H}$ & -4.23061500 & 3.81664900 & -0.33210000 \\
\hline $\mathrm{H}$ & -3.22460000 & 5.22229500 & -0.10052500 \\
\hline $\mathrm{C}$ & -3.66214700 & 4.80630400 & -2.19323500 \\
\hline $\mathrm{H}$ & -4.54760700 & 5.45043200 & -2.22189400 \\
\hline $\mathrm{H}$ & -2.81 & 3100 & -2. \\
\hline $\mathrm{H}$ & -3.84036400 & 3.94882400 & -2.84902200 \\
\hline $\mathrm{P}$ & -2.61353900 & -0.80819200 & -0.20082200 \\
\hline $\mathrm{H}$ & -3.19207400 & 0.32946100 & -0.80612700 \\
\hline $\mathrm{C}$ & -3.46877400 & -0.81919600 & 1.41719600 \\
\hline $\mathrm{C}$ & -3.93284200 & -2.01194700 & 1.99291700 \\
\hline $\mathrm{C}$ & -3.56759500 & 0.38600500 & 2.13554300 \\
\hline $\mathrm{C}$ & -4.50704200 & -1.99747800 & 3.26686100 \\
\hline $\mathrm{H}$ & -3.85655500 & -2.94912300 & 1.44942300 \\
\hline $\mathrm{C}$ & -4.14559600 & 0.39073700 & 3.40666600 \\
\hline $\mathrm{H}$ & -3.18682000 & 1.31329300 & 1.71228500 \\
\hline $\mathrm{C}$ & -4.61694800 & -0.79745500 & 3.97466900 \\
\hline $\mathrm{H}$ & -4.86862400 & -2.92434400 & 3.70313800 \\
\hline $\mathrm{H}$ & -4.22356600 & 1.32609300 & 3.95346900 \\
\hline $\mathrm{H}$ & -5.06452100 & -0.78815200 & 4.96440300 \\
\hline $\mathrm{C}$ & -3.31930000 & -2.17770500 & -1.19420400 \\
\hline $\mathrm{C}$ & -2.47102200 & -3.18340500 & -1.67877800 \\
\hline $\mathrm{C}$ & -4.69143300 & -2.22326700 & -1.49876100 \\
\hline $\mathrm{C}$ & -2.98924200 & -4.22747400 & -2.45201800 \\
\hline $\mathrm{H}$ & -1.40766200 & -3.14134500 & -1.46157400 \\
\hline $\mathrm{C}$ & -5.20357000 & -3.26365200 & -2.27472500 \\
\hline $\mathrm{H}$ & -5.35864700 & -1.45015800 & -1.12763500 \\
\hline $\mathrm{C}$ & -4.35266900 & -4.26816000 & -2.75062400 \\
\hline
\end{tabular}

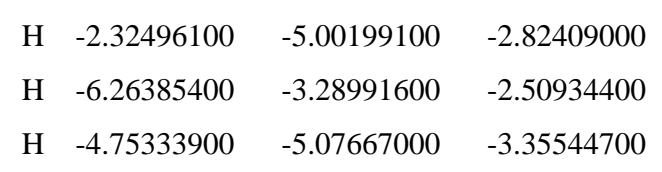

C-i/F-IM2

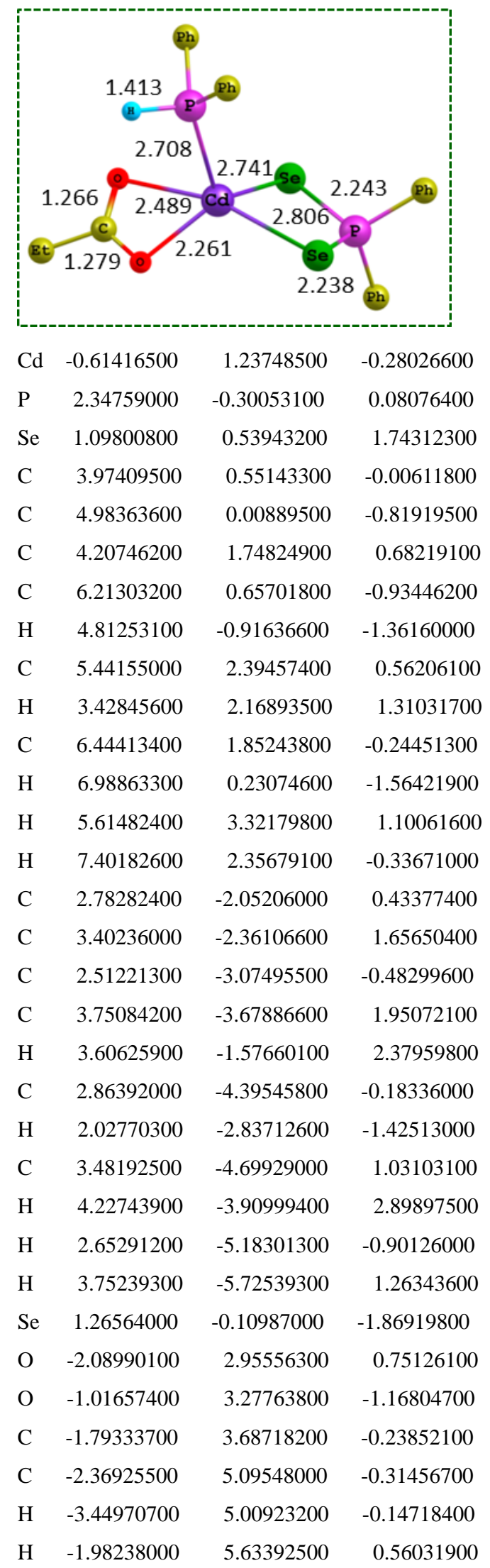




$\begin{array}{cccc}\mathrm{C} & -2.06554500 & 5.86078400 & -1.60353400 \\ \mathrm{H} & -2.50680600 & 6.86300400 & -1.56521600 \\ \mathrm{H} & -0.98772000 & 5.96291300 & -1.75669100 \\ \mathrm{H} & -2.47040300 & 5.34155200 & -2.47746400 \\ \mathrm{P} & -2.97428600 & -0.09055700 & -0.31383000 \\ \mathrm{H} & -3.99512100 & 0.79048400 & -0.73570500 \\ \mathrm{C} & -3.57367500 & -0.54499500 & 1.36809500 \\ \mathrm{C} & -3.92976800 & -1.85756600 & 1.70824200 \\ \mathrm{C} & -3.61798200 & 0.46480200 & 2.34613800 \\ \mathrm{C} & -4.33756100 & -2.15737600 & 3.01187400 \\ \mathrm{H} & -3.89390800 & -2.64656000 & 0.96367100 \\ \mathrm{C} & -4.03525400 & 0.15723300 & 3.64270300 \\ \mathrm{H} & -3.32152400 & 1.48046800 & 2.09636000 \\ \mathrm{C} & -4.39455100 & -1.15190700 & 3.97955400 \\ \mathrm{H} & -4.61093600 & -3.17753500 & 3.26654100 \\ \mathrm{H} & -4.07105600 & 0.94305300 & 4.39188300 \\ \mathrm{H} & -4.71252600 & -1.38682800 & 4.99137200 \\ \mathrm{C} & -3.29970900 & -1.52790000 & -1.40119100 \\ \mathrm{C} & -2.23360300 & -2.36339200 & -1.77126000 \\ \mathrm{C} & -4.58930700 & -1.80061700 & -1.89122500 \\ \mathrm{C} & -2.45828000 & -3.46332100 & -2.60448600 \\ \mathrm{H} & -1.22635900 & -2.14580800 & -1.42729600 \\ \mathrm{C} & -4.80815000 & -2.89574200 & -2.72883800 \\ \mathrm{H} & -5.42266100 & -1.15937300 & -1.61713400 \\ \mathrm{C} & -3.74294500 & -3.73067600 & -3.08354100 \\ \mathrm{H} & -1.62637000 & -4.10185000 & -2.88689500 \\ \mathrm{H} & -5.80741800 & -3.09591500 & -3.10481500 \\ \mathrm{H} & -3.91437600 & -4.58159800 & -3.73665000\end{array}$

E-ii

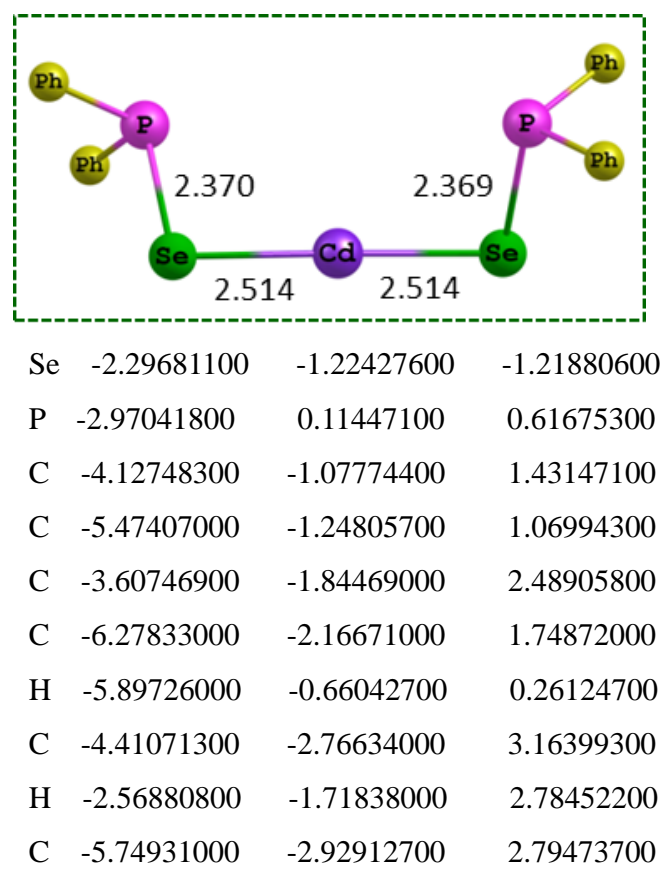

$\begin{array}{lrrr}\mathrm{H} & -7.31859400 & -2.28755000 & 1.45891600 \\ \mathrm{H} & -3.99349600 & -3.35183800 & 3.97853300 \\ \mathrm{H} & -6.37743900 & -3.64242800 & 3.32094800 \\ \mathrm{C} & -4.15175500 & 1.33958300 & -0.12461300 \\ \mathrm{C} & -4.57951500 & 2.36457200 & 0.74031200 \\ \mathrm{C} & -4.59804100 & 1.35230800 & -1.45350200 \\ \mathrm{C} & -5.45146100 & 3.35945200 & 0.29414200 \\ \mathrm{H} & -4.22932400 & 2.38549100 & 1.77010200 \\ \mathrm{C} & -5.46356300 & 2.35540500 & -1.90325700 \\ \mathrm{H} & -4.26411900 & 0.58088000 & -2.14059700 \\ \mathrm{C} & -5.89565000 & 3.35824700 & -1.03220400 \\ \mathrm{H} & -5.77419200 & 4.13940100 & 0.97826300 \\ \mathrm{H} & -5.79910200 & 2.34961400 & -2.93685400 \\ \mathrm{H} & -6.56676500 & 4.13667200 & -1.38405200 \\ \mathrm{Cd} & 0.00654600 & -0.22399600 & -1.33115000 \\ \mathrm{Se} & 2.30622100 & 0.77911400 & -1.48534600 \\ \mathrm{P} & 2.99230200 & -0.14574900 & 0.58508600 \\ \mathrm{C} & 3.87461900 & 1.31254300 & 1.30424900 \\ \mathrm{C} & 5.16024000 & 1.72565400 & 0.91606600 \\ \mathrm{C} & 3.19835300 & 2.04279000 & 2.29612700 \\ \mathrm{C} & 5.75274500 & 2.84326800 & 1.50668400 \\ \mathrm{H} & 5.70024300 & 1.17095900 & 0.15460900 \\ \mathrm{C} & 3.79020900 & 3.16366700 & 2.88471000 \\ \mathrm{H} & 6.08593000 & -2.77483500 & -2.42444700 \\ \mathrm{H} & 7.32496000 & -3.77177800 & -0.50982800 \\ \mathrm{C} & 5.06912600 & 3.56584800 & 2.49068000 \\ \mathrm{H} & 6.74755400 & 3.15191700 & 1.19718000 \\ \mathrm{H} & 3.25509400 & 3.71736400 & 3.65115600 \\ \mathrm{H} & 5.53270100 & 4.43509100 & 2.94870100 \\ \mathrm{C} & 4.41769600 & -1.24325300 & 0.12753200 \\ \mathrm{C} & 5.11681800 & -1.82423400 & 1.20367500 \\ \mathrm{C} & 4.77668100 & -1.60442600 & -1.17806900 \\ \mathrm{C} & 6.16048000 & -2.72183200 & 0.97665000 \\ \mathrm{H} & 4.84844300 & -1.57003000 & 2.22660800 \\ \mathrm{H} & 5.81949600 & -2.51044600 & -1.40462600 \\ & 6.51572500 & -3.06932000 & -0.33158200 \\ & & \\ \mathrm{H} & & \end{array}$

$\begin{array}{llll}\text { H } & 7.32496000 & -3.77177800 & -0.50982800\end{array}$ 


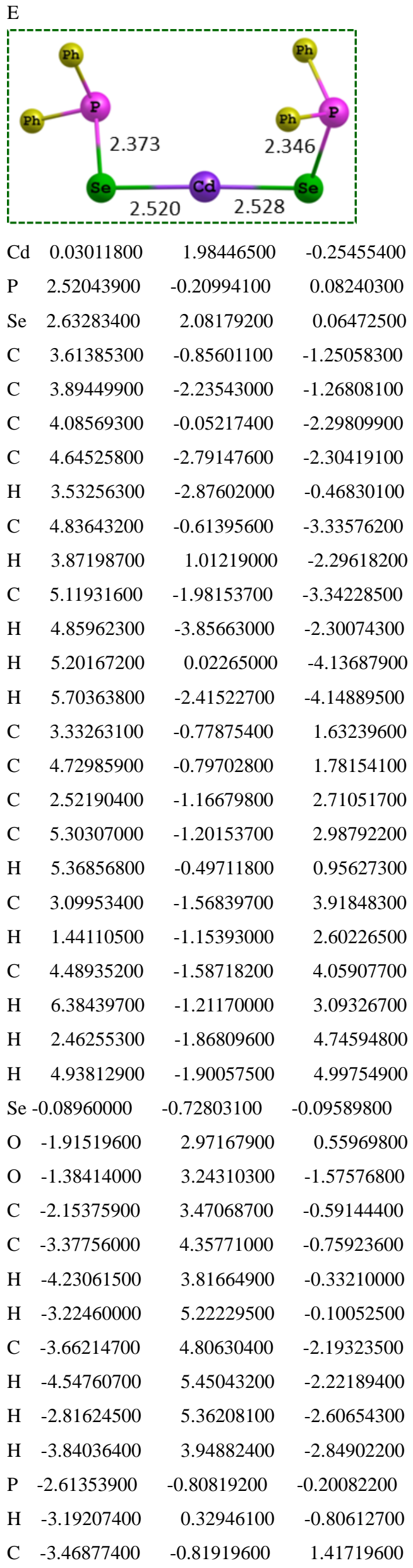

$\begin{array}{lrrr}\mathrm{C} & -3.93284200 & -2.01194700 & 1.99291700 \\ \mathrm{C} & -3.56759500 & 0.38600500 & 2.13554300 \\ \mathrm{C} & -4.50704200 & -1.99747800 & 3.26686100 \\ \mathrm{H} & -3.85655500 & -2.94912300 & 1.44942300 \\ \mathrm{C} & -4.14559600 & 0.39073700 & 3.40666600 \\ \mathrm{H} & -3.18682000 & 1.31329300 & 1.71228500 \\ \mathrm{C} & -4.61694800 & -0.79745500 & 3.97466900 \\ \mathrm{H} & -4.86862400 & -2.92434400 & 3.70313800 \\ \mathrm{H} & -4.22356600 & 1.32609300 & 3.95346900 \\ \mathrm{H} & -5.06452100 & -0.78815200 & 4.96440300 \\ \mathrm{C} & -3.31930000 & -2.17770500 & -1.19420400 \\ \mathrm{C} & -2.47102200 & -3.18340500 & -1.67877800 \\ \mathrm{C} & -4.69143300 & -2.22326700 & -1.49876100 \\ \mathrm{C} & -2.98924200 & -4.22747400 & -2.45201800 \\ \mathrm{H} & -1.40766200 & -3.14134500 & -1.46157400 \\ \mathrm{C} & -5.20357000 & -3.26365200 & -2.27472500 \\ \mathrm{H} & -5.35864700 & -1.45015800 & -1.12763500 \\ \mathrm{C} & -4.35266900 & -4.26816000 & -2.75062400 \\ \mathrm{H} & -2.32496100 & -5.00199100 & -2.82409000 \\ \mathrm{H} & -6.26385400 & -3.28991600 & -2.50934400 \\ \mathrm{H} & -4.75333900 & -5.07667000 & -3.35544700\end{array}$

F

\begin{tabular}{|cccc|}
\hline & & & \\
\hline
\end{tabular}




$\begin{array}{lrrr}\mathrm{C} & -2.45969900 & -2.46908300 & 0.74958400 \\ \mathrm{C} & -4.58614000 & -2.25608600 & -1.05730800 \\ \mathrm{H} & -3.75665900 & -0.34602700 & -1.58940900 \\ \mathrm{C} & -3.39952300 & -3.49877100 & 0.64749500 \\ \mathrm{H} & -1.63531700 & -2.54908900 & 1.45113900 \\ \mathrm{C} & -4.46125000 & -3.39480200 & -0.25381800 \\ \mathrm{H} & -5.40930800 & -2.17086200 & -1.76061500 \\ \mathrm{H} & -3.29911400 & -4.37952900 & 1.27487600 \\ \mathrm{H} & -5.18989100 & -4.19656400 & -0.33150500 \\ \mathrm{O} & 3.89891300 & -1.35428100 & -0.04343600 \\ \mathrm{O} & 3.97806700 & 0.86053100 & 0.00621900 \\ \mathrm{C} & 4.56768700 & -0.26621700 & -0.02342100 \\ \mathrm{C} & 6.08380100 & -0.34790800 & -0.04269000 \\ \mathrm{H} & 6.37415000 & -1.03295400 & 0.76276500 \\ \mathrm{H} & 6.35573200 & -0.86242200 & -0.97309800 \\ \mathrm{C} & 6.80756500 & 0.99372100 & 0.07721500 \\ \mathrm{H} & 7.89198800 & 0.84291000 & 0.04819700 \\ \mathrm{H} & 6.53088400 & 1.66679700 & -0.73899500 \\ \mathrm{H} & 6.55611200 & 1.49640800 & 1.01563500\end{array}$

\section{C/G-IM1}

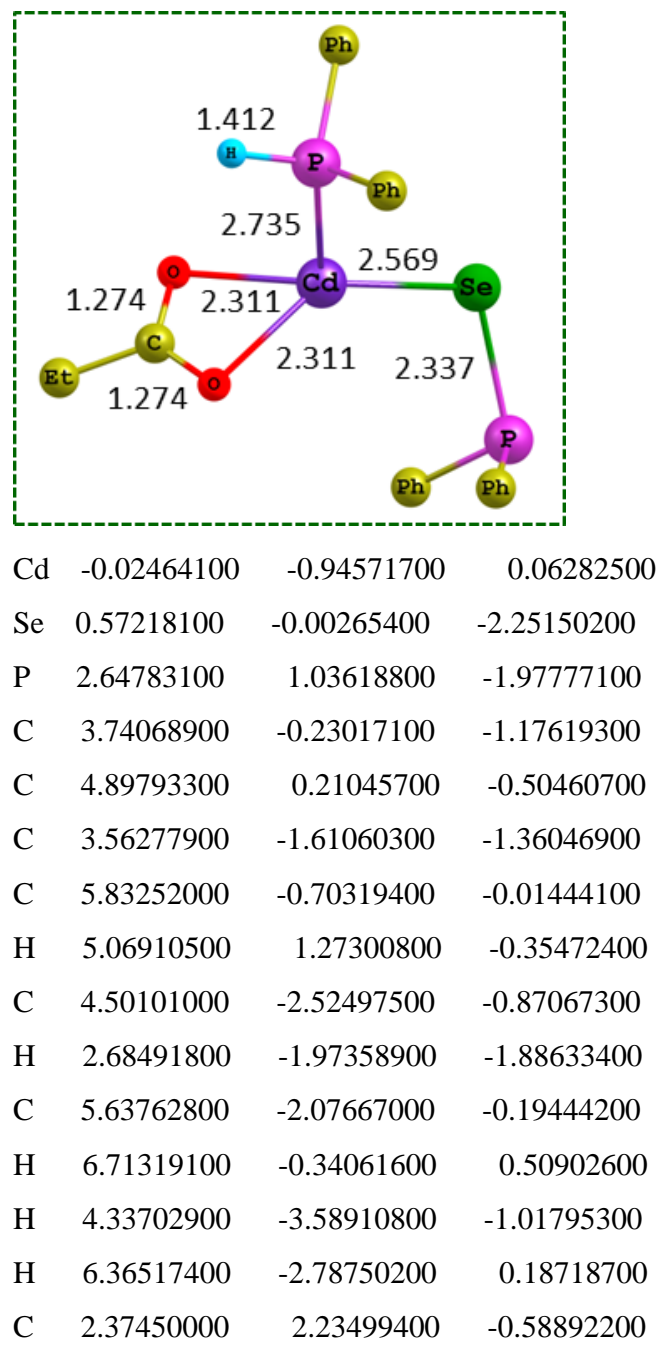

\begin{tabular}{|c|c|c|c|}
\hline C & 2.27429900 & 1.88295400 & 8800 \\
\hline $\mathrm{C}$ & 2.24136600 & 3.58766100 & -0.94579300 \\
\hline $\mathrm{C}$ & 2.03906800 & 2.85870400 & 1.73937500 \\
\hline $\mathrm{H}$ & 2.37921000 & 0.84779700 & 1.08011400 \\
\hline $\mathrm{C}$ & 2.00574400 & 4.56568500 & 0.02617000 \\
\hline 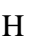 & 2.32419300 & 3.87512400 & -1.99115900 \\
\hline $\mathrm{C}$ & 1.90325700 & 4.20243000 & 1.37140200 \\
\hline $\mathrm{H}$ & 1.95984900 & 2.56565800 & 2.78260500 \\
\hline $\mathrm{H}$ & 1.90971900 & 5.60752700 & -0.26793400 \\
\hline $\mathrm{H}$ & 1.72241500 & 4.95970700 & 2.12939300 \\
\hline$P$ & -2.62910400 & -0.42342000 & 0.71248500 \\
\hline $\mathrm{H}$ & -3.05811600 & -0.9 & 1.94 \\
\hline $\mathrm{C}$ & -3.04627700 & 1.35122000 & 0.92865900 \\
\hline$c$ & -2.33415900 & 2.31139700 & 0.19230400 \\
\hline$C$ & -4.05564000 & 1.76811500 & 1.81314600 \\
\hline $\mathrm{C}$ & -2.63843200 & 3.66869900 & 0.33067300 \\
\hline $\mathrm{H}$ & -1.53966700 & 2.00807000 & -0.48488600 \\
\hline $\mathrm{C}$ & -4.34966600 & 3.12512800 & 1.95449700 \\
\hline $\mathrm{H}$ & -4.61262600 & 1.03648500 & 2.39216900 \\
\hline $\mathrm{C}$ & -3.64342300 & 4.07668200 & 1.21052600 \\
\hline $\mathrm{H}$ & -2.07962000 & 4.40272300 & -0.24195900 \\
\hline $\mathrm{H}$ & -5.12830800 & 3.43906700 & 2.64380300 \\
\hline $\mathrm{H}$ & -3.87344600 & 5.13244300 & 1.32176600 \\
\hline $\mathrm{C}$ & -3.90678100 & -1.13607800 & -0.39574100 \\
\hline $\mathrm{C}$ & -4.96347300 & -1.91225000 & 0.10640000 \\
\hline $\mathrm{C}$ & -3.80328000 & -0.92927800 & -1.78167700 \\
\hline $\mathrm{C}$ & -5.90713000 & -2.46435600 & -0.76401200 \\
\hline $\mathrm{H}$ & -5.05203200 & -2.09089600 & 1.17444900 \\
\hline $\mathrm{C}$ & -4.75430400 & -1.47357500 & -2.64665500 \\
\hline $\mathrm{H}$ & -2.98097300 & -0.34677400 & -2.18956100 \\
\hline $\mathrm{C}$ & -5.80674200 & -2.24238900 & -2.13994900 \\
\hline $\mathrm{H}$ & -6.71923200 & -3.06566500 & -0.36549200 \\
\hline 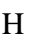 & -4.66629500 & -1.30475500 & -3.71581900 \\
\hline $\mathrm{H}$ & -6.54155200 & -2.67108100 & -2.81509400 \\
\hline $\mathrm{O}$ & 0.21293100 & -2.91418600 & 1.24982400 \\
\hline 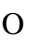 & 0.92825500 & -1.02509100 & 2.16718400 \\
\hline $\mathrm{C}$ & 0.77187100 & -2.28879400 & 2.20888800 \\
\hline $\mathrm{C}$ & 1.25231800 & -3.08476700 & 3.41149600 \\
\hline $\mathrm{H}$ & 0.38255600 & -3.63024300 & 3.79868100 \\
\hline $\mathrm{H}$ & 1.92904800 & -3.85644300 & 3.02465400 \\
\hline S & 1.92480900 & -2.26220400 & 4.51140800 \\
\hline $\mathrm{H}$ & 2.23962700 & -2.91306700 & 5.33431000 \\
\hline $\mathrm{H}$ & 2.80676200 & -1.74024400 & 4.12953600 \\
\hline $\mathrm{H}$ & 1.24439900 & -1.50544100 & 4.912783 \\
\hline
\end{tabular}




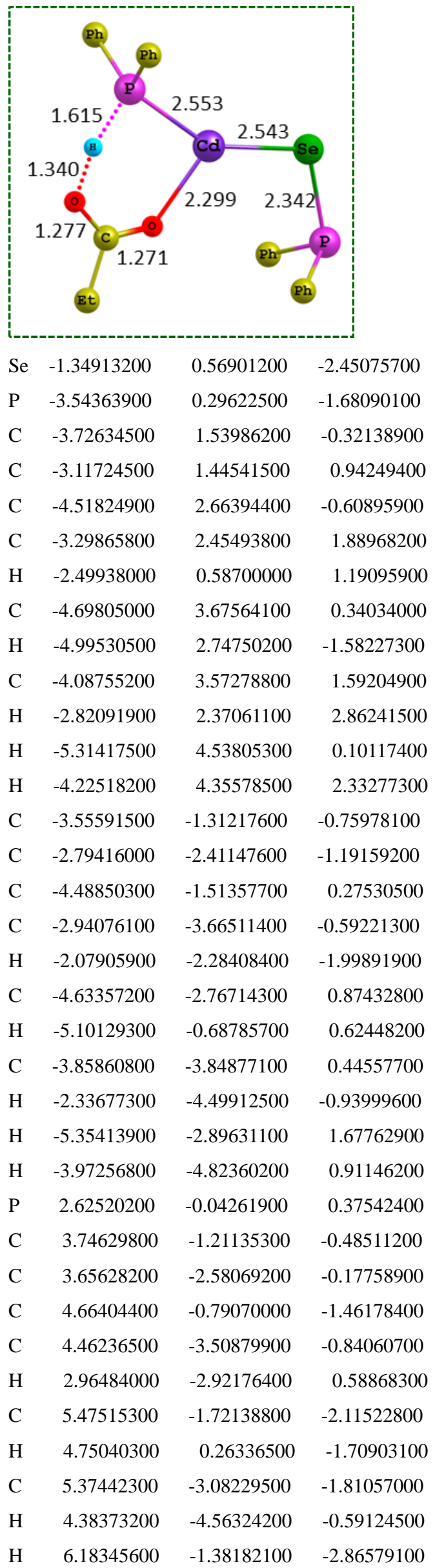

$\begin{array}{lrrr}\mathrm{H} & 6.00446900 & -3.80403300 & -2.32220000 \\ \mathrm{C} & 3.50662400 & 1.55855000 & 0.51911400 \\ \mathrm{C} & 4.54187200 & 1.69886200 & 1.46217000 \\ \mathrm{C} & 3.15226800 & 2.66565300 & -0.26946900 \\ \mathrm{C} & 5.21009500 & 2.91607200 & 1.60371400 \\ \mathrm{H} & 4.82215000 & 0.85520900 & 2.08655200 \\ \mathrm{C} & 3.81945500 & 3.88587900 & -0.12244300 \\ \mathrm{H} & 2.35520300 & 2.57761300 & -1.00269300 \\ \mathrm{C} & 4.84937700 & 4.01224400 & 0.81250100 \\ \mathrm{H} & 6.00696500 & 3.01046700 & 2.33594200 \\ \mathrm{H} & 3.53278800 & 4.73319800 & -0.73862700 \\ \mathrm{H} & 5.36719700 & 4.96011900 & 0.92762100 \\ \mathrm{Cd} & 0.23795700 & 0.11131300 & -0.51731100 \\ \mathrm{H} & 2.07916400 & -0.64348300 & 1.77134300 \\ \mathrm{C} & -0.85156700 & -1.58342200 & 3.67798800 \\ \mathrm{H} & -1.48537400 & -0.73815000 & 3.97568600 \\ \mathrm{H} & -1.53314500 & -2.30134600 & 3.20590200 \\ \mathrm{O} & 1.32574300 & -1.13407700 & 2.76429100 \\ \mathrm{C} & 0.06470100 & -1.06724300 & 2.57526600 \\ \mathrm{O} & -0.50313300 & -0.60041000 & 1.53893200 \\ \mathrm{C} & -0.14938200 & -2.19488100 & 4.89077300 \\ \mathrm{H} & 0.51289500 & -1.47094600 & 5.37374200 \\ \mathrm{H} & -0.88891600 & -2.52936800 & 5.62615500 \\ \mathrm{H} & 0.46083500 & -3.05631200 & 4.60388400\end{array}$

C/G-IM2

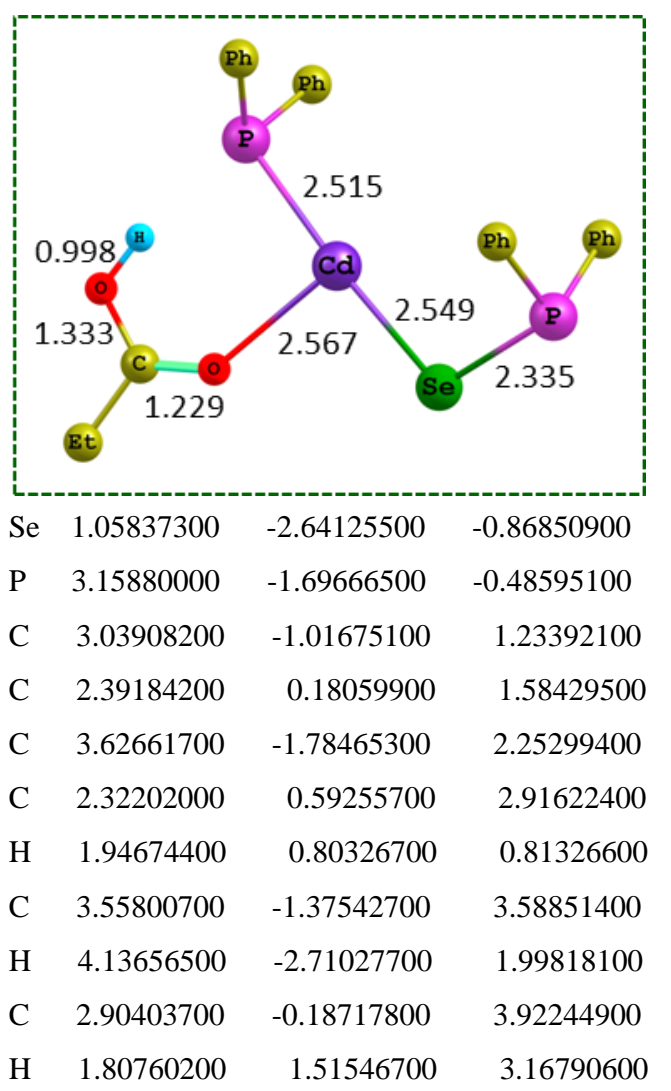




\begin{tabular}{|c|c|c|c|}
\hline$H$ & 4.01676500 & 2300 & 00 \\
\hline $\mathrm{H}$ & 2.84782200 & 0.13327200 & 4.95905900 \\
\hline $\mathrm{C}$ & 3.22172900 & -0.15220500 & -1.51375500 \\
\hline $\mathrm{C}$ & 4.15126200 & 0.85121900 & -1.17507400 \\
\hline $\mathrm{C}$ & 2.52448600 & -0.02894400 & -2.72690900 \\
\hline $\mathrm{C}$ & 4.35733000 & 1.94989900 & -2.01157900 \\
\hline $\mathrm{H}$ & 4.71666600 & 0.77888200 & -0.25010200 \\
\hline $\mathrm{C}$ & 2.73634400 & 1.06889900 & -3.56748200 \\
\hline $\mathrm{H}$ & 1.81392100 & -0.79751500 & -3.01583100 \\
\hline $\mathrm{C}$ & 3.65055300 & 2.06410300 & -3.21343100 \\
\hline $\mathrm{H}$ & 5.07306600 & 2.71554800 & -1.72439500 \\
\hline $\mathrm{H}$ & 2.18544600 & 1.14076700 & -4.50165400 \\
\hline $\mathrm{H}$ & 3.81673500 & 2.91548400 & -3.86756000 \\
\hline $\mathrm{P}$ & -2.18358500 & 1.05120800 & 1800 \\
\hline $\mathrm{C}$ & -1.54598800 & 1.84960700 & 1.88397800 \\
\hline $\mathrm{C}$ & -1.75325600 & 1.17923500 & 3.10526900 \\
\hline $\mathrm{C}$ & -0.92590200 & 3.11025100 & 1.91329500 \\
\hline $\mathrm{C}$ & -1.34539200 & 1.74485900 & 4.31344700 \\
\hline $\mathrm{H}$ & -2.24458600 & 0.20855000 & 3.11337900 \\
\hline $\mathrm{C}$ & -0.52712700 & 3.68097300 & 3.12667000 \\
\hline $\mathrm{H}$ & -0.75870600 & 3.65433700 & 0.98916000 \\
\hline $\mathrm{C}$ & -0.73201000 & 3.00265900 & 4.3309 \\
\hline $\mathrm{H}$ & -1.51498300 & 1.20729400 & 5.24244600 \\
\hline $\mathrm{H}$ & -0.05522000 & 4.66006300 & 3.12613500 \\
\hline $\mathrm{H}$ & -0.42353000 & 3.44924100 & 5.27181600 \\
\hline $\mathrm{C}$ & -1.94468000 & 2.32009700 & -0.97944100 \\
\hline $\mathrm{C}$ & -3.05649800 & 3.10145100 & -1.34609300 \\
\hline $\mathrm{C}$ & -0.72947800 & 2.53348500 & -1.65490500 \\
\hline $\mathrm{C}$ & -2.95445100 & 4.07112600 & -2.34760200 \\
\hline $\mathrm{H}$ & -4.00714300 & 2.94746100 & -0.84281 \\
\hline $\mathrm{C}$ & -0.62690100 & 3.49933500 & -2.65995000 \\
\hline $\mathrm{H}$ & 0.14712100 & 1.94548500 & -1.39836900 \\
\hline $\mathrm{C}$ & -1.73872800 & 4.27194500 & -3.00731100 \\
\hline $\mathrm{H}$ & -3.82500600 & 4.66405000 & -2.61420000 \\
\hline $\mathrm{H}$ & 0.32236400 & 3.64352000 & -3.16783400 \\
\hline $\mathrm{H}$ & -1.65917100 & 5.02195900 & -3.78921600 \\
\hline $\mathrm{Cd}$ & -0.63761700 & -0.84821000 & -0.22956700 \\
\hline $\mathrm{H}$ & -3.90647400 & -0.43871200 & 0.25358100 \\
\hline $\mathrm{C}$ & -4.84038600 & -3.49229100 & -0.37168200 \\
\hline $\mathrm{H}$ & -5.38372700 & -3.60073900 & 0.57551200 \\
\hline $\mathrm{H}$ & -5.59939100 & -3.19898000 & -1.10816900 \\
\hline $\mathrm{O}$ & -4.53967800 & -1.20405800 & 0.15787900 \\
\hline $\mathrm{C}$ & -3.90355900 & -2.31530100 & -0.21240800 \\
\hline $\mathrm{O}$ & -2.69097100 & -2.37963400 & -0.40423700 \\
\hline $\mathrm{C}$ & -4.14388900 & -4.79201000 & -0.77211300 \\
\hline $\mathrm{H}$ & -3.40154100 & -5.09004500 & -0.02661600 \\
\hline
\end{tabular}

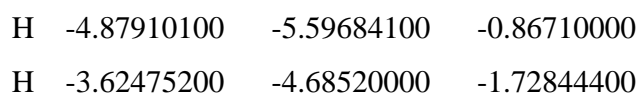

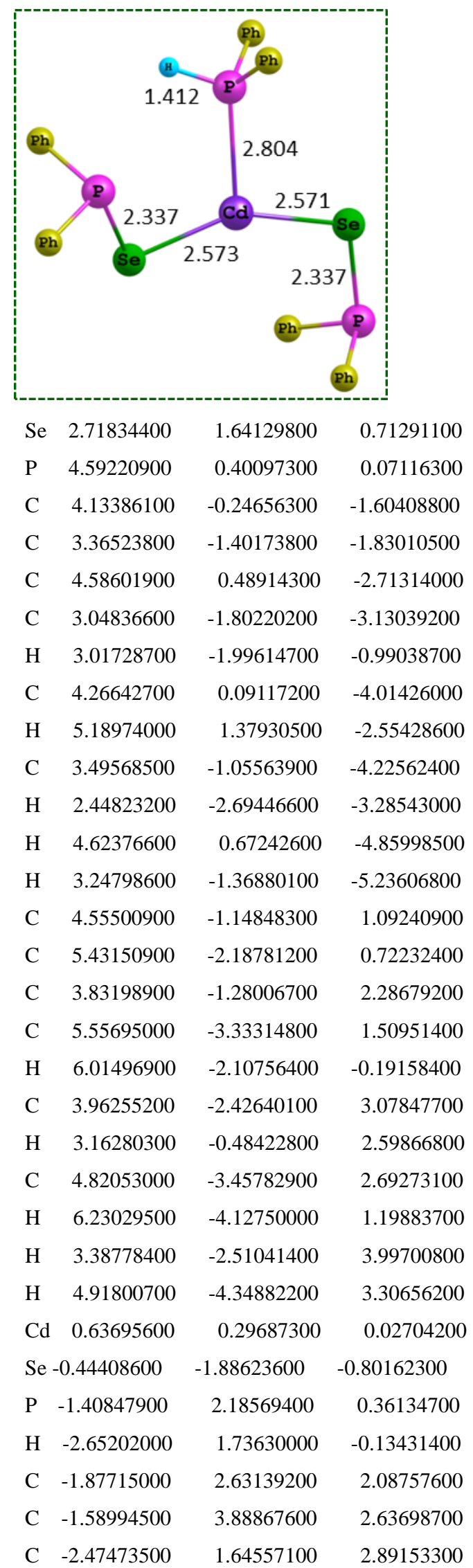




\begin{tabular}{|c|c|c|c|}
\hline$C$ & -1.90606900 & 4.15969000 & 3.97188500 \\
\hline $\mathrm{H}$ & -1.12692900 & 4.65965400 & 2.02891200 \\
\hline C & -2.79460100 & 1.92518200 & 4.22129400 \\
\hline & -2.68895100 & 0.66208000 & 2.47912800 \\
\hline C & -2.51001800 & 3.18192100 & 4.76561600 \\
\hline H & -1.68033200 & 5.13764800 & 4.38743300 \\
\hline $\mathrm{H}$ & -3.26367700 & 1.15919300 & 4.83241400 \\
\hline $\mathrm{H}$ & -2.75560100 & 3.39562500 & 5.80182100 \\
\hline $\mathrm{C}$ & -1.24042900 & 3.76405100 & -0.55567600 \\
\hline $\mathrm{C}$ & -2.36846900 & 4.42761400 & -1.07021200 \\
\hline $\mathrm{C}$ & 0.03496300 & 4.31573700 & -0.76072600 \\
\hline $\mathrm{C}$ & -2.22152100 & 5.62599000 & -1.77087300 \\
\hline $\mathrm{H}$ & -3.36047300 & 4.00974400 & -0.92294400 \\
\hline C & 0.17609500 & 5.52108100 & -1.45511200 \\
\hline $\mathrm{H}$ & 0.92083800 & 3.80646200 & -0.38987800 \\
\hline $\mathrm{C}$ & -0.94925600 & 6.17595800 & -1.96111200 \\
\hline $\mathrm{H}$ & -3.09879200 & 6.12869400 & -2.16789300 \\
\hline $\mathrm{H}$ & 1.16708500 & 5.93898700 & -1.60609700 \\
\hline $\mathrm{H}$ & -0.83679900 & 7.10856100 & -2.50661800 \\
\hline 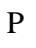 & -2.53267300 & -1.58465300 & 0.20342900 \\
\hline $\mathrm{C}$ & -3.00195900 & -3.34531100 & 0.52940000 \\
\hline $\mathrm{C}$ & -3.47514600 & -4.22571700 & -0.45780000 \\
\hline 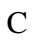 & -2.86084700 & -3.81727900 & 1.84513000 \\
\hline $\mathrm{C}$ & -3.80069800 & -5.54442100 & -0.13403900 \\
\hline $\mathrm{H}$ & -3.59143200 & -3.87961400 & -1.48040400 \\
\hline$C$ & -3.18216400 & -5.13829100 & 2.16859900 \\
\hline $\mathrm{H}$ & -2.49560000 & -3.14720600 & 2.61953900 \\
\hline $\mathrm{C}$ & -3.65438900 & -6.00461300 & 1.17897300 \\
\hline $\mathrm{H}$ & -4.16593300 & -6.21404000 & -0.90804200 \\
\hline $\mathrm{H}$ & -3.06696200 & -5.48761200 & 3.19099500 \\
\hline $\mathrm{H}$ & -3.90800600 & -7.03118000 & 1.42833100 \\
\hline $\mathrm{C}$ & -3.68283500 & -1.15570900 & -1.19371600 \\
\hline $\mathrm{C}$ & -5.04530900 & -1.01740300 & -0.86303900 \\
\hline $\mathrm{C}$ & -3.28098400 & -0.87027500 & -2.50591800 \\
\hline $\mathrm{C}$ & -5.97920100 & -0.62476700 & -1.82268000 \\
\hline П & -5.38048100 & -1.22461400 & 0.15104800 \\
\hline $\mathrm{C}$ & -4.21670100 & -0.46778300 & -3.46672500 \\
\hline $\mathrm{H}$ & -2.23439200 & -0.97167500 & -2.77767700 \\
\hline C & -5.56655400 & -0.34626200 & -3.13106500 \\
\hline $\mathrm{H}$ & -7.02697500 & -0.53368500 & -1.54917100 \\
\hline $\mathrm{H}$ & -3.88565200 & -0.25568500 & -4.47997600 \\
\hline $\mathrm{H}$ & -6.29161900 & -0.03643200 & -3.87845500 \\
\hline
\end{tabular}

E/G-TS1

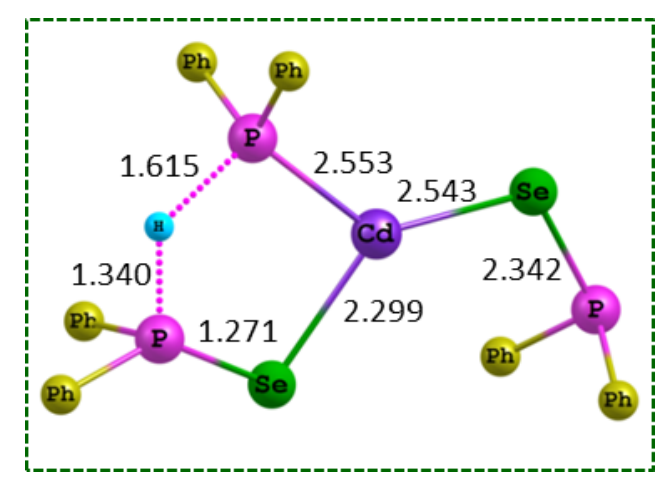

Se $\quad-2.71797400 \quad-2.37865700 \quad 0.00516200$

P $\quad-4.59852300 \quad-1.00592900 \quad-0.12992800$

C $\quad-4.34236200 \quad-0.00357200 \quad-1.66657800$

C $\quad-3.63373200 \quad 1.20776300 \quad-1.73418800$

C $\quad-4.91292000 \quad-0.51512000-2.84545700$

C $\quad-3.49205800 \quad 1.88276000 \quad-2.94915400$

$\begin{array}{llll}\mathrm{H} & -3.19593700 & 1.63247900 & -0.83621100\end{array}$

C $\quad-4.76635500 \quad 0.15571000 \quad-4.06228700$

$\mathrm{H} \quad-5.47187100 \quad-1.44686500 \quad-2.80789900$

$\begin{array}{llll}\text { C } & -4.05488700 & 1.35768800 & -4.11688400\end{array}$

$\mathrm{H} \quad-2.93552300 \quad 2.81536700 \quad-2.98225900$

H $\quad-5.21208200 \quad-0.25693100 \quad-4.96327900$

$\mathrm{H} \quad-3.94155200 \quad 1.88338000 \quad-5.06097100$

C $\quad-4.42176700 \quad 0.26944000 \quad 1.20629400$

C $\quad-5.26948600 \quad 1.39435500 \quad 1.16112000$

$\begin{array}{llll}\text { C } & -3.62282600 & 0.08633000 & 2.34509300\end{array}$

$\begin{array}{lrrr}\text { C } & -5.29388100 & 2.31685100 & 2.20813200\end{array}$

$\mathrm{H} \quad-5.91164500 \quad 1.55613400 \quad 0.29922100$

C $\quad-3.65230000 \quad 1.00758600 \quad 3.39772100$

$\begin{array}{llll}\mathrm{H} & -2.97848800 & -0.78501400 & 2.41048200\end{array}$

$\begin{array}{llll}\text { C } & -4.48352900 & 2.12779500 & 3.33311900\end{array}$

$\mathrm{H} \quad-5.94850500 \quad 3.18209500 \quad 2.14590900$

$\mathrm{H} \quad-3.02363800 \quad 0.84424500 \quad 4.26934400$

$\mathrm{H} \quad-4.50673900 \quad 2.84291100 \quad 4.15076100$

$\begin{array}{lll}\text { Cd }-0.55237400 & -1.01049800 & -0.16388800\end{array}$

Se $-0.11187300 \quad 1.60868600 \quad-0.83416600$

$\begin{array}{llll}\text { P } & 1.90521600 & -1.62898100 & 0.29677000\end{array}$

$\begin{array}{llll}\mathrm{H} & 2.33634700 & 0.15374200 & 0.28768700\end{array}$

$\begin{array}{llll}\text { C } & 2.22274900 & -2.55726100 & 1.84904500\end{array}$

$\begin{array}{llll}\text { C } & 1.24155500 & -3.38483200 & 2.42383800\end{array}$

$\begin{array}{llll}\text { C } & 3.45476200 & -2.41141400 & 2.51425900\end{array}$

C $\quad 1.48845700 \quad-4.05272300 \quad 3.62686800$

$\mathrm{H} \quad 0.28255800 \quad-3.50944900 \quad 1.92924100$

$\begin{array}{llll}\text { C } & 3.70337800 & -3.08480200 & 3.71262600\end{array}$

$\mathrm{H} \quad 4.22112400 \quad-1.76786700 \quad 2.09088700$

$\begin{array}{llll}\text { C } & 2.71924600 & -3.90573200 & 4.27241100\end{array}$ 


\begin{tabular}{|c|c|c|c|}
\hline & 0.71836000 & -4.68720900 & 4.05647000 \\
\hline & 4.66106200 & -2.96283400 & 4.21114400 \\
\hline & 2.90986000 & -4.42504800 & 5.20737200 \\
\hline & 2.66882100 & -2.56717400 & -1.08000400 \\
\hline & 3.40775000 & -3.74801000 & -0.88749800 \\
\hline & 2.53520700 & -2.06562600 & -2.39099800 \\
\hline & 3.99486600 & -4.40381400 & -1.97322100 \\
\hline & 3.52101100 & -4.16045300 & 0.11000900 \\
\hline & 3.12400900 & -2.72395400 & -3.47124500 \\
\hline & 1.95946700 & -1.16044700 & -2.57274600 \\
\hline & 3.85882300 & -3.89723500 & -3.26853800 \\
\hline & 4.55687400 & -5.31825600 & -1.80263100 \\
\hline & 3.00209700 & -2.32192100 & -4.47343100 \\
\hline & 4.31328700 & -4.41184800 & -4.11002100 \\
\hline $\mathrm{P}$ & 1.96950700 & 1.75232200 & 0.02424700 \\
\hline & 1.99629700 & 2.84957400 & 1.49229300 \\
\hline C & 0.82451200 & 3.14897300 & 2.20341900 \\
\hline $\mathrm{C}$ & 3.23365000 & 3.31393500 & 1.97758200 \\
\hline & 0.88825400 & 3.90788600 & 3.37542000 \\
\hline & -0.13446400 & 2.80148600 & 1.83166500 \\
\hline & 3.29041400 & 4.07149500 & 3.14796000 \\
\hline & 4.15074100 & 3.09300200 & 1.43871200 \\
\hline $\mathrm{C}$ & 2.11776900 & 4.37061300 & 3.85033500 \\
\hline $\mathrm{r}$ & -0.02726000 & 4.14002800 & 3.91194200 \\
\hline & 4.25018500 & 4.42956700 & 3.50956000 \\
\hline & 2.16418400 & 4.96178200 & 4.76037100 \\
\hline c & 3.10913100 & 2.50700600 & -1.18920100 \\
\hline $\mathrm{C}$ & 4.03674100 & 1.68264000 & -1.84685200 \\
\hline C & 3.06093600 & 3.87674900 & -1.49912300 \\
\hline C & 4.90735000 & 2.22285100 & -2.79789100 \\
\hline $\mathrm{H}$ & 4.08210400 & 0.62076900 & -1.62215700 \\
\hline $\mathrm{C}$ & 3.93184300 & 4.41142200 & -2.44880500 \\
\hline $\mathrm{H}$ & 2.34390000 & 4.52195800 & -1.00012000 \\
\hline $\mathrm{C}$ & 4.85594500 & 3.58574300 & -3.09958300 \\
\hline $\mathrm{H}$ & 5.62252500 & 1.57760100 & -3.29971800 \\
\hline $\mathrm{H}$ & 3.88742900 & 5.47099200 & -2.68400400 \\
\hline & 5.53215900 & 4.00499000 & -3.83910200 \\
\hline
\end{tabular}

E/G-IM2

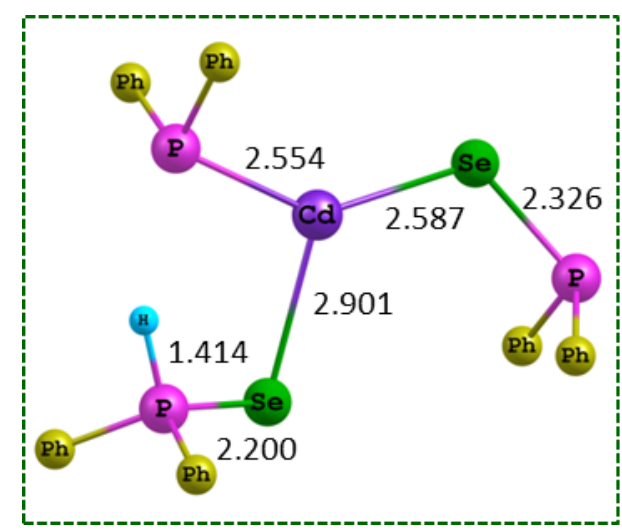

$\begin{array}{llll}\text { Se } & -2.36398700 & -2.50649300 & 0.15796900\end{array}$

$\begin{array}{llll}\mathrm{P} & -4.30029700 & -1.23300500 & -0.03720000\end{array}$

$\begin{array}{llll}\text { C } & -4.17083800 & -0.39960400 & -1.68668500\end{array}$

$\begin{array}{llll}\text { C } & -3.59723200 & 0.86075100 & -1.92197800\end{array}$

$\begin{array}{llll}\text { C } & -4.70619400 & -1.10677600 & -2.77823300\end{array}$

$\begin{array}{llll}\text { C } & -3.55155000 & 1.39352800 & -3.21309200\end{array}$

$\begin{array}{llll}\mathrm{H} & -3.19015100 & 1.43461000 & -1.09575100\end{array}$

$\begin{array}{llll}\text { C } & -4.65331700 & -0.57936300 & -4.07052900\end{array}$

$\begin{array}{llll}\mathrm{H} & -5.16270900 & -2.07948700 & -2.61264000\end{array}$

$\begin{array}{llll}\text { C } & -4.07552900 & 0.67429900 & -4.29174400\end{array}$

$\begin{array}{llll}\mathrm{H} & -3.09889400 & 2.36815100 & -3.37479000\end{array}$

$\begin{array}{llll}\mathrm{H} & -5.06823800 & -1.14388500 & -4.90112900\end{array}$

$\begin{array}{llll}\mathrm{H} & -4.03614700 & 1.08892500 & -5.29525400\end{array}$

$\begin{array}{llll}\text { C } & -4.14299300 & 0.18350100 & 1.15103900\end{array}$

$\begin{array}{lrrr}\text { C } & -5.05428900 & 1.25277100 & 1.03701700\end{array}$

$\begin{array}{llll}\text { C } & -3.29168200 & 0.15537100 & 2.26632900\end{array}$

$\begin{array}{llll}\text { C } & -5.08871800 & 2.27174500 & 1.99031900\end{array}$

$\begin{array}{llll}\mathrm{H} & -5.74102300 & 1.29379700 & 0.19558200\end{array}$

$\begin{array}{llll}\text { C } & -3.33215400 & 1.17180900 & 3.22686600\end{array}$

$\begin{array}{llll}\mathrm{H} & -2.59926300 & -0.67224900 & 2.38684200\end{array}$

$\begin{array}{llll}\text { C } & -4.22643600 & 2.23620000 & 3.09187700\end{array}$

$\begin{array}{llll}\mathrm{H} & -5.79447200 & 3.09021900 & 1.87551300\end{array}$

$\begin{array}{llll}\mathrm{H} & -2.66538200 & 1.12394700 & 4.08415000\end{array}$

$\begin{array}{llll}\mathrm{H} & -4.26259500 & 3.02299700 & 3.84055000\end{array}$

$\begin{array}{lll}\mathrm{Cd}-0.14503600 & -1.18745300 & -0.00758900\end{array}$

Se $-0.21253100 \quad 1.54190100 \quad-0.98716800$

$\begin{array}{llll}\text { P } & 2.34513900 & -1.53543500 & 0.44011400\end{array}$

$\begin{array}{llll}\mathrm{H} & 2.35410100 & 1.13031800 & 0.47593400\end{array}$

$\begin{array}{llll}\text { C } & 2.37572900 & -2.63543900 & 1.93372100\end{array}$

$\begin{array}{llll}\text { C } & 1.46943200 & -3.68884000 & 2.16000400\end{array}$

$\begin{array}{llll}\text { C } & 3.34673700 & -2.37623300 & 2.91777900\end{array}$

$\begin{array}{llll}\text { C } & 1.53484800 & -4.45625500 & 3.32494800\end{array}$

$\begin{array}{llll}\mathrm{H} & 0.70434500 & -3.91140600 & 1.42155100\end{array}$

$\begin{array}{llll}\text { C } & 3.41300500 & -3.14101400 & 4.08760600\end{array}$

$\begin{array}{llll}\mathrm{H} & 4.05865800 & -1.56980900 & 2.76202400\end{array}$ 


\begin{tabular}{|c|c|c|c|}
\hline $\mathrm{C}$ & 2.50643400 & -4.18323000 & 4.29413000 \\
\hline $\mathrm{H}$ & 0.82142200 & -5.26132200 & 3.47834400 \\
\hline $\mathrm{H}$ & 4.17208600 & -2.92130700 & 4.83371800 \\
\hline $\mathrm{H}$ & 2.55305100 & -4.77705000 & 8200 \\
\hline C & 2.79948400 & -2.65691300 & -0.96282400 \\
\hline $\mathrm{C}$ & 3.34410400 & -3.94123100 & -0.78681500 \\
\hline $\mathrm{C}$ & 2.68673100 & -2.16236200 & -2.27872100 \\
\hline $\mathrm{C}$ & 3.75180800 & -4.70402400 & -1.88607900 \\
\hline $\mathrm{H}$ & 3.44944900 & -4.35263000 & 3800 \\
\hline $\mathrm{C}$ & 3.09 & -2.92590600 & -3.37396900 \\
\hline $\mathrm{H}$ & 2.27097400 & -1.17120800 & -2.45123500 \\
\hline $\mathrm{C}$ & 3.62712500 & -4.20468600 & -3.18447300 \\
\hline $\mathrm{H}$ & 4.16601500 & -5.69553600 & -1.72157900 \\
\hline $\mathrm{H}$ & 2.98199200 & -2.52277200 & -4.37756500 \\
\hline $\mathrm{H}$ & 3.93980600 & -4.80137400 & -4.03650300 \\
\hline $\mathrm{P}$ & 1.64343800 & 2.24088900 & -0.03580600 \\
\hline $\mathrm{C}$ & 1.37890700 & 3.40141300 & 1.35293200 \\
\hline $\mathrm{C}$ & 0.114 & 3.52302 & 456100 \\
\hline $\mathrm{C}$ & 2.46508500 & 4.13447100 & 1.86201700 \\
\hline $\mathrm{C}$ & -0.06174900 & 4.37360500 & 3.03940100 \\
\hline $\mathrm{H}$ & -0.72660900 & 2.96195700 & 1.54860300 \\
\hline $\mathrm{C}$ & 2.28145300 & 4.97955300 & 2.95712900 \\
\hline $\mathrm{H}$ & 3.446 & 4.05333100 & 1.40467800 \\
\hline $\mathrm{C}$ & 1.01810000 & 5.10026100 & 3.54621600 \\
\hline $\mathrm{H}$ & -1.04508900 & 4.46623400 & 3.49033100 \\
\hline $\mathrm{H}$ & 3.12272800 & 5.54492400 & 3.34709000 \\
\hline $\mathrm{H}$ & 0.87758800 & 5.76127700 & 4.39659200 \\
\hline $\mathrm{C}$ & 2.84827600 & 2.99341600 & -1.16904300 \\
\hline $\mathrm{C}$ & 4.03047000 & 600 & -1.46774200 \\
\hline $\mathrm{C}$ & 2.58710000 & 4.22987000 & -1.78097200 \\
\hline $\mathrm{C}$ & 4.95114900 & 2.84625600 & -2.36517000 \\
\hline $\mathrm{H}$ & 4.23187100 & 1.33378300 & -1.00853800 \\
\hline $\mathrm{C}$ & 3.51076800 & 4.77060000 & -2.67511500 \\
\hline $\mathrm{H}$ & 1.66799200 & 4.76624100 & 4500 \\
\hline $\mathrm{C}$ & 4.69262700 & 4.07978000 & -2.96771800 \\
\hline $\mathrm{H}$ & 5.86503600 & 2.30610500 & -2.59356200 \\
\hline $\mathrm{H}$ & 3.30761700 & 5.72767400 & -3.14629700 \\
\hline $\mathrm{H}$ & 5.40836800 & 4.50265700 & -3.66665200 \\
\hline
\end{tabular}

F/E-i-TS1a

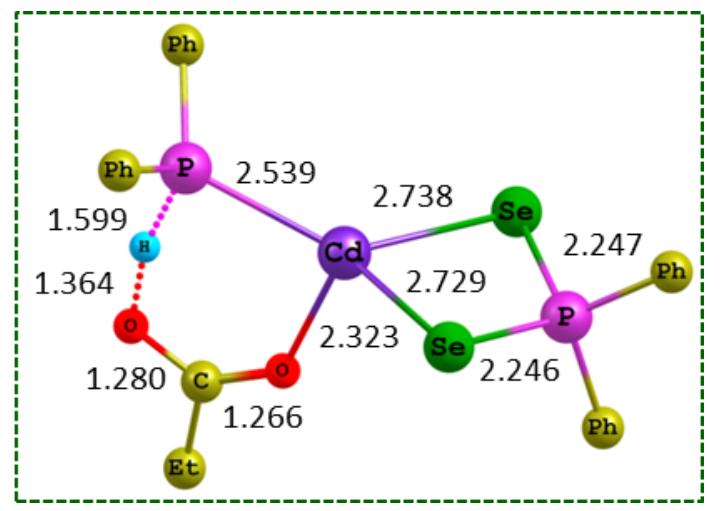

\begin{tabular}{|c|c|c|c|}
\hline $\mathrm{Cd}$ & 0.62319800 & 0.22256800 & -0.10700700 \\
\hline $\mathrm{O}$ & 0.73166500 & 2.50524400 & -0.52361000 \\
\hline $\mathrm{C}$ & 1.70871000 & 3.28454700 & -0.72371000 \\
\hline $\mathrm{C}$ & 1.42967200 & 4.76169600 & -0.98955700 \\
\hline $\mathrm{H}$ & 1.97229600 & 5.32625400 & -0.22083700 \\
\hline $\mathrm{H}$ & 1.92624400 & 5.01022300 & -1.93553300 \\
\hline $\mathrm{C}$ & -0.04659800 & 5.15648800 & -1.02093900 \\
\hline $\mathrm{H}$ & -0.14849300 & 6.23044300 & -1.21193800 \\
\hline $\mathrm{H}$ & -0.58274600 & 4.61708000 & -1.80759500 \\
\hline $\mathrm{H}$ & -0.53630000 & 4.92967600 & -0.06925000 \\
\hline $\mathrm{O}$ & 2.94539000 & 2.95333000 & -0.72274500 \\
\hline $\mathrm{H}$ & 3.22599800 & 1.63445700 & -0.51631000 \\
\hline $\mathrm{P}$ & -2.60365500 & -0.39347800 & 0.07006300 \\
\hline & -1.35503200 & -0.66735400 & -1.77769500 \\
\hline $\mathrm{Se}-$ & -1.26264200 & -0.07041300 & 1.84305700 \\
\hline $\mathrm{C}$ & -3.69144600 & -1.84703500 & 0.33192000 \\
\hline $\mathrm{C}$ & -4.02963800 & -2.25544100 & 1.63051100 \\
\hline $\mathrm{C}$ & -4.20698200 & -2.54343100 & -0.77241100 \\
\hline $\mathrm{C}$ & -4.88851300 & -3.34101600 & 1.82033600 \\
\hline $\mathrm{H}$ & -3.61443000 & -1.73689200 & 2.48912300 \\
\hline $\mathrm{C}$ & -5.06496100 & -3.62758700 & -0.57652100 \\
\hline $\mathrm{H}$ & -3.93024100 & -2.24855400 & -1.78003200 \\
\hline $\mathrm{C}$ & -5.40768300 & -4.02698400 & 0.71893600 \\
\hline $\mathrm{H}$ & -5.14333800 & -3.65327900 & 2.82888600 \\
\hline $\mathrm{H}$ & -5.45772900 & -4.16335200 & -1.43578200 \\
\hline $\mathrm{H}$ & -6.07030400 & -4.87458900 & 0.86899700 \\
\hline $\mathrm{C}$ & -3.71948400 & 1.04787100 & -0.14171000 \\
\hline $\mathrm{C}$ & -5.11265700 & 0.92339400 & -0.04496000 \\
\hline $\mathrm{C}$ & -3.14819000 & 2.30558800 & -0.40045300 \\
\hline $\mathrm{C}$ & -5.92805600 & 2.04837100 & -0.20785400 \\
\hline $\mathrm{H}$ & -5.56707200 & -0.04079400 & 0.15573500 \\
\hline $\mathrm{C}$ & -3.96801800 & 3.42118800 & -0.56138400 \\
\hline $\mathrm{H}$ & -2.06991800 & 2.41517300 & -0.47539400 \\
\hline $\mathrm{C}$ & -5.35906800 & 3.29624100 & -0.46621500 \\
\hline $\mathrm{H}$ & -7.00655700 & 1.94323000 & -0.13215200 \\
\hline
\end{tabular}




$\begin{array}{lrrr}\mathrm{H} & -3.51896600 & 4.38956200 & -0.76143000 \\ \mathrm{H} & -5.99374100 & 4.16869000 & -0.59295000 \\ \mathrm{P} & 3.15678400 & 0.07506000 & -0.16821700 \\ \mathrm{C} & 3.99527300 & -0.24127100 & 1.43167400 \\ \mathrm{C} & 4.28257600 & 0.84539400 & 2.27615500 \\ \mathrm{C} & 4.32672100 & -1.53968400 & 1.85455700 \\ \mathrm{C} & 4.88222400 & 0.63440000 & 3.52071900 \\ \mathrm{H} & 4.04631800 & 1.85705700 & 1.95737800 \\ \mathrm{C} & 4.93647300 & -1.74493100 & 3.09374700 \\ \mathrm{H} & 4.11295400 & -2.39039100 & 1.21371100 \\ \mathrm{C} & 5.21260000 & -0.65974800 & 3.93205500 \\ \mathrm{H} & 5.09935200 & 1.48358300 & 4.16264100 \\ \mathrm{H} & 5.19102400 & -2.75386100 & 3.40647000 \\ \mathrm{H} & 5.68402100 & -0.82230900 & 4.89705300 \\ \mathrm{C} & 3.90918300 & -1.05472300 & -1.40111700 \\ \mathrm{C} & 5.28323600 & -0.96203600 & -1.69129700 \\ \mathrm{C} & 3.12927800 & -1.98890300 & -2.10164300 \\ \mathrm{C} & 5.86049700 & -1.79059600 & -2.65407300 \\ \mathrm{H} & 5.90031500 & -0.24060600 & -1.16313200 \\ \mathrm{C} & 3.70854300 & -2.81244200 & -3.07259700 \\ \mathrm{H} & 2.06781200 & -2.07952900 & -1.88806500 \\ \mathrm{C} & 5.07398200 & -2.71670900 & -3.34881400 \\ \mathrm{H} & 6.92264300 & -1.70865200 & -2.86725600 \\ \mathrm{H} & 3.09128700 & -3.52970100 & -3.60604300 \\ \mathrm{H} & 5.52473900 & -3.35795100 & -4.10089400\end{array}$

F/E-i-IM2a

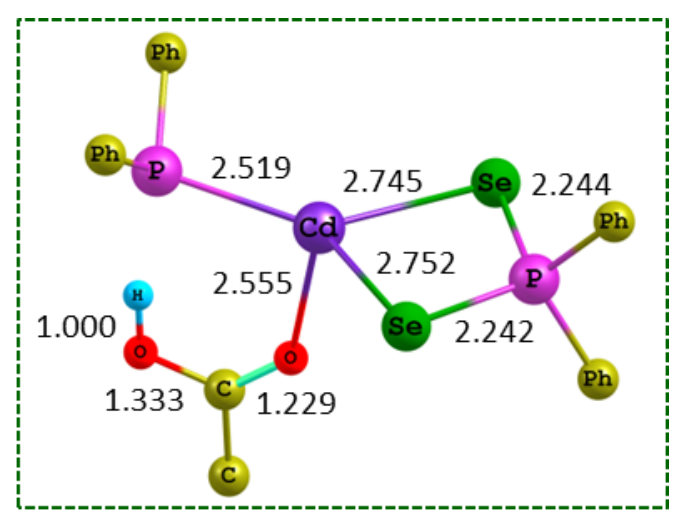

$\begin{array}{lrrr}\mathrm{Cd} & 0.87473500 & 0.29587600 & -0.09863000 \\ \mathrm{O} & 0.91705400 & 2.76317800 & -0.76234000 \\ \mathrm{C} & 1.76412700 & 3.64981000 & -0.84163000 \\ \mathrm{C} & 1.43521500 & 5.09528400 & -1.14772400 \\ \mathrm{H} & 1.78973700 & 5.68796600 & -0.29456200 \\ \mathrm{H} & 2.06372500 & 5.39957100 & -1.99366000 \\ \mathrm{C} & -0.04529500 & 5.35088500 & -1.42515700 \\ \mathrm{H} & -0.21270000 & 6.41491500 & -1.61902400 \\ \mathrm{H} & -0.38615500 & 4.78554200 & -2.29734200\end{array}$

\begin{tabular}{|c|c|c|c|}
\hline & 00 & 00 & 00 \\
\hline & 3.07061800 & .45688100 & 6642020 \\
\hline & 0 & 0 & 454345 \\
\hline & .41995900 & -0.18188000 & 66900 \\
\hline & 00 & -0.5 & 200 \\
\hline & 00 & .36778200 & 00 \\
\hline & -3.41782400 & -1.67854300 & 0400 \\
\hline & -3.15 & -2.4 & \\
\hline & -4.42829200 & -2.06528600 & 72700 \\
\hline C & -3.8 & -3.62463100 & 9900 \\
\hline & -2.37014000 & -2.17223000 & 1000 \\
\hline & -5.16957100 & -3.22 & -0 \\
\hline $\mathrm{H}$ & -4.63 & -1.46754200 & 100 \\
\hline & -4.90650400 & -4.00476 & 00 \\
\hline $\mathrm{H}$ & -3.69002300 & -4.22855500 & 500 \\
\hline $\mathrm{H}$ & -5.94974300 & -3.51472300 & 500 \\
\hline $\mathrm{H}$ & -5.48 & 40 & 800 \\
\hline & -3.65256800 & 0100 & 0500 \\
\hline $\mathrm{C}$ & -4.78094900 & 1.25287600 & 9100 \\
\hline $\mathrm{C}$ & -3.4 & 0 & 00 \\
\hline & -5.70848000 & 2.27346400 & 2700 \\
\hline $\mathrm{H}$ & -4 & 0 & 1.37124200 \\
\hline $\mathrm{C}$ & -4.39200200 & 3.08493200 & -1.51492200 \\
\hline & -2.58 & 00 & 700 \\
\hline $\mathrm{C}$ & -5.51641400 & 3.19060500 & 3900 \\
\hline & -6.57892800 & 100 & 0.99063300 \\
\hline $\mathrm{H}$ & -4.23 & 0 & 0100 \\
\hline $\mathrm{H}$ & -6.23960600 & 3.98418200 & -0.85783600 \\
\hline$P$ & 3.39182500 & .28814400 & 300 \\
\hline C & 3.86162400 & -0.47746900 & 800 \\
\hline & 3.56334200 & 2300 & 41900 \\
\hline $\mathrm{C}$ & 4.55289600 & -1.69586000 & 1.71270600 \\
\hline$C$ & 0 & 00 & 900 \\
\hline $\mathrm{H}$ & 100 & & 800 \\
\hline $\mathrm{C}$ & 0266 & 10160 & 00 \\
\hline 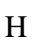 & 4.80182400 & -2.26253400 & 25400 \\
\hline $\mathrm{C}$ & 4.61660400 & -1.48540400 & 4.13045000 \\
\hline $\mathrm{H}$ & 0 & 0 & 50 \\
\hline $\mathrm{H}$ & .46046900 & 00 & 1300 \\
\hline $\mathrm{H}$ & U & 00 & 0 \\
\hline$C_{1}-$ & 800 & 00 & 1400 \\
\hline $\mathrm{C}$ & 5.15807600 & 8500 & 58600 \\
\hline$c$ & 3.20186500 & -2.08506300 & -1.63601900 \\
\hline $\mathrm{C}$ & 5.63327700 & -1.49032600 & -2 \\
\hline $\mathrm{H}$ & 5.71598600 & 37900 & 202900 \\
\hline & 3.73381200 & -2.94286500 & 296400 \\
\hline
\end{tabular}




$\begin{array}{llll}\mathrm{H} & 2.33730000 & -2.32778700 & -1.11970500 \\ \mathrm{C} & 4.92124100 & -2.64730700 & -3.30995400 \\ \mathrm{H} & 6.55584500 & -1.25124800 & -3.50539700 \\ \mathrm{H} & 3.17140100 & -3.83851500 & -2.88220900 \\ \mathrm{H} & 5.28615500 & -3.31228300 & -4.08774100\end{array}$

F/E-i-TS1b

\begin{tabular}{|c|c|c|c|}
\hline & $\mathrm{Ph}$ & & h \\
\hline $\mathrm{Cd}$ & 0.55705700 & -0.17796400 & 0.05342400 \\
\hline $\mathrm{O}$ & 0.02517800 & 3.75624800 & -0.27328700 \\
\hline $\mathrm{C}$ & 1.18055100 & 3.36072300 & -0.18258200 \\
\hline $\mathrm{C}$ & 2.38525300 & 4.27769400 & -0.38966300 \\
\hline $\mathrm{H}$ & 3.00280200 & 4.22199900 & 0.51652400 \\
\hline $\mathrm{H}$ & 2.99694100 & 3.84165900 & -1.19099800 \\
\hline $\mathrm{C}$ & 2.01800000 & 5.72579400 & -0.71202600 \\
\hline $\mathrm{H}$ & 2.92194800 & 6.32846000 & -0.85206500 \\
\hline $\mathrm{H}$ & 1.41790900 & 5.78444400 & -1.62447600 \\
\hline $\mathrm{H}$ & 1.42796600 & 6.16982900 & 0.09478000 \\
\hline $\mathrm{O}$ & 1.48494900 & 2.10270200 & 0.10559800 \\
\hline $\mathrm{H}$ & 2.63687800 & 1.45439400 & 0.03792000 \\
\hline $\mathrm{P}$ & -2.71392200 & -0.37750500 & 0.03734500 \\
\hline $\mathrm{Se}$ & -1.40353400 & -0.41685000 & -1.79533700 \\
\hline $\mathrm{Se}$ & -1.41225600 & -0.55996300 & 1.86753400 \\
\hline $\mathrm{C}$ & -3.93068900 & -1.74707000 & -0.03909600 \\
\hline $\mathrm{C}$ & -4.20307800 & -2.53689900 & 1.08600500 \\
\hline $\mathrm{C}$ & -4.60561600 & -1.99853000 & -1.24550200 \\
\hline $\mathrm{C}$ & -5.15453900 & -3.55845600 & 1.00922000 \\
\hline $\mathrm{H}$ & -3.66843700 & -2.36195400 & 2.01428500 \\
\hline $\mathrm{C}$ & -5.55354800 & -3.01977000 & -1.31631200 \\
\hline $\mathrm{H}$ & -4.38700600 & -1.40570400 & -2.12872000 \\
\hline $\mathrm{C}$ & -5.83017500 & -3.80075200 & -0.18886400 \\
\hline $\mathrm{H}$ & -5.35953200 & -4.16661700 & 1.88542700 \\
\hline $\mathrm{H}$ & -6.06979600 & -3.20922700 & -2.25288200 \\
\hline $\mathrm{H}$ & -6.56495500 & -4.59864000 & -0.24760800 \\
\hline $\mathrm{C}$ & -3.68439200 & 1.17346500 & 0.09554000 \\
\hline $\mathrm{C}$ & -5.07375400 & 1.15666100 & 0.29676700 \\
\hline $\mathrm{C}$ & -3.00875000 & 2.39906200 & -0.03190400 \\
\hline $\mathrm{C}$ & -5.78241100 & 2.35962300 & 0.36421100 \\
\hline
\end{tabular}

$\begin{array}{lrrr}\mathrm{H} & -5.60608200 & 0.21726700 & 0.40021300 \\ \mathrm{C} & -3.72577900 & 3.59334800 & 0.03819700 \\ \mathrm{H} & -1.93347400 & 2.44661200 & -0.18117600 \\ \mathrm{C} & -5.11092400 & 3.57754100 & 0.23435200 \\ \mathrm{H} & -6.85762800 & 2.33966100 & 0.51774000 \\ \mathrm{H} & -3.19057100 & 4.53269100 & -0.06128300 \\ \mathrm{H} & -5.66423600 & 4.51113900 & 0.28633300 \\ \mathrm{P} & 3.10485600 & -0.11490500 & 0.03549900 \\ \mathrm{C} & 4.02389700 & -0.64868300 & 1.52487700 \\ \mathrm{C} & 3.88289900 & 0.09807900 & 2.70885100 \\ \mathrm{C} & 4.83469000 & -1.79589100 & 1.53761600 \\ \mathrm{C} & 4.53505900 & -0.29792300 & 3.87797000 \\ \mathrm{H} & 3.26388000 & 0.99167500 & 2.71683300 \\ \mathrm{C} & 5.49373700 & -2.18232300 & 2.70740400 \\ \mathrm{H} & 4.95422600 & -2.38696900 & 0.63461000 \\ \mathrm{C} & 5.34488200 & -1.43808600 & 3.88122500 \\ \mathrm{H} & 4.41524100 & 0.28934500 & 4.78407900 \\ \mathrm{H} & 6.12008800 & -3.07014900 & 2.70062000 \\ \mathrm{H} & 5.8500 \\ \mathrm{H} & 5.85567600 & -1.74266000 & 4.79004400 \\ \mathrm{C} & 3.99373300 & -0.73707400 & -1.43872400 \\ \mathrm{C} & 5.32171300 & -0.33912300 & -1.68421600 \\ \mathrm{C} & 3.35399900 & -1.57113600 & -2.37026300 \\ \mathrm{H} & 5.99141100 & -0.77314200 & -2.82887900 \\ \mathrm{H} & 5.83092100 & 0.31032900 & -0.97762400 \\ \mathrm{H} & 4.02272300 & -1.99438300 & -3.52339500 \\ \mathrm{H} & 2.33234800 & -1.89528000 & -2.19389000 \\ \mathrm{H} & 5.34225100 & -1.59943100 & -3.75333300 \\ \mathrm{H} & -3.003551300 & -0.45971300 & -3.03500 \\ \mathrm{H} & -2.63670300 & -4.23517900 \\ \mathrm{H} & -1.93155500 & -4.64666800\end{array}$

F/E-i-IM2b

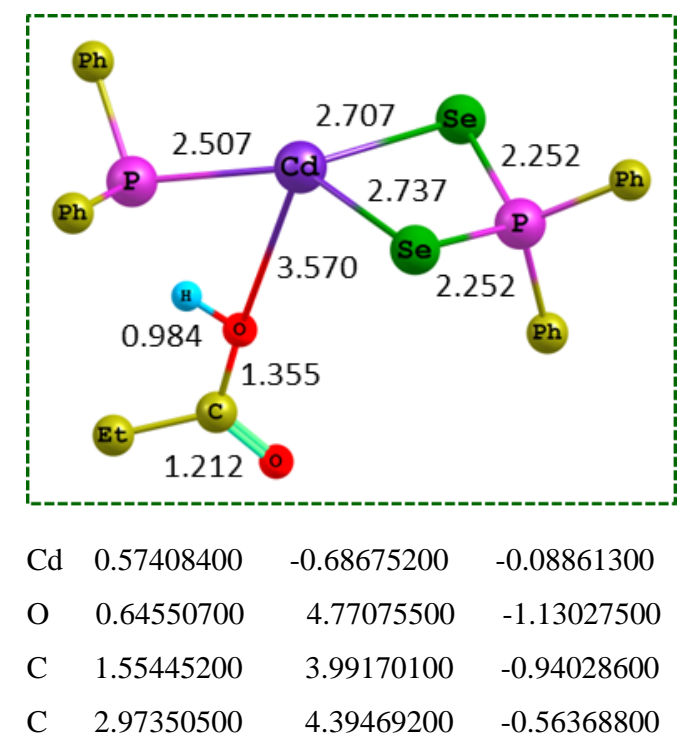




\begin{tabular}{|c|c|c|c|}
\hline $\mathrm{H}$ & 3.24943500 & 3.85463700 & 0.35183800 \\
\hline $\mathrm{H}$ & 3.64539900 & 4.01426500 & -1.34515600 \\
\hline C & 3.14791100 & 5.90249700 & -0.38406900 \\
\hline $\mathrm{H}$ & 4.18454300 & 6.13451100 & -0.12062700 \\
\hline $\mathrm{H}$ & 2.89401900 & 6.43864000 & -1.30238000 \\
\hline $\mathrm{H}$ & 2.49618200 & 6.28070400 & 0.40829300 \\
\hline $\mathrm{O}$ & 1.33453900 & 2.66095700 & -1.06714800 \\
\hline $\mathrm{H}$ & 2.12735900 & 2.11544700 & -0.86215200 \\
\hline $\mathrm{P}$ & -2.67588300 & -0.32807600 & 0.02695200 \\
\hline $\mathrm{S}$ & -1.50090100 & -0.86393100 & -1.81838300 \\
\hline $\mathrm{Se}$ & -1.37554600 & -0.67543800 & 1.83251300 \\
\hline $\mathrm{C}$ & -4.20549500 & -1.33147400 & 0.09746100 \\
\hline $\mathrm{C}$ & -4.42651100 & -2.25305500 & 1.12851100 \\
\hline $\mathrm{C}$ & -5.14789900 & -1.20293400 & -0.93790800 \\
\hline $\mathrm{C}$ & -5.58902300 & -3.03024900 & 1.13235300 \\
\hline $\mathrm{H}$ & -3.69203300 & -2.36538200 & 1.91994800 \\
\hline $\mathrm{C}$ & -6.30423700 & -1.98224500 & -0.92920800 \\
\hline $\mathrm{H}$ & -4.98019100 & -0.49988700 & -1.74869700 \\
\hline $\mathrm{C}$ & -6.52705200 & -2.89671300 & 0.10674100 \\
\hline $\mathrm{H}$ & -5.75580800 & -3.74061700 & 9700 \\
\hline $\mathrm{H}$ & -7.02851000 & -1.87788200 & -1.73172700 \\
\hline $\mathrm{H}$ & -7.42789000 & -3.50365800 & 0.11059900 \\
\hline $\mathrm{C}$ & -3.20589300 & 1.42394600 & -0.02649200 \\
\hline $\mathrm{C}$ & -4.42575400 & 1.81686200 & 0.54834100 \\
\hline $\mathrm{C}$ & -2.34433600 & 2.394 & 43600 \\
\hline $\mathrm{C}$ & -4.77831400 & 3.16819600 & 0.57606500 \\
\hline $\mathrm{H}$ & -5.09909000 & 1.07928900 & 0.97299800 \\
\hline $\mathrm{C}$ & -2.69643700 & 3.74428400 & -0.51981300 \\
\hline $\mathrm{H}$ & -1.39782000 & 2.11072200 & -1.00967400 \\
\hline $\mathrm{C}$ & -3.91563300 & 4.13162500 & 0.04498900 \\
\hline $\mathrm{H}$ & -5.72570400 & 3.46495400 & 1.01705200 \\
\hline $\mathrm{H}$ & -2.00509700 & 4.47815800 & -0.92272500 \\
\hline $\mathrm{H}$ & -4.19134200 & 5.18203800 & 0.07413900 \\
\hline $\mathrm{P}$ & 2.99141500 & -0.02781600 & -0.16204200 \\
\hline $\mathrm{C}$ & 3.62528800 & -0.33485600 & 1.54662600 \\
\hline $\mathrm{C}$ & 2.95566300 & 0.26127400 & 2.63352 \\
\hline $\mathrm{C}$ & 4.81529900 & -1.03687100 & 1.80510600 \\
\hline $\mathrm{C}$ & 3.45308700 & 0.15115000 & 3.93231800 \\
\hline $\mathrm{H}$ & 2.03204800 & 0.81213500 & 2.46786900 \\
\hline $\mathrm{C}$ & 5.31161600 & -1.14690800 & 3.10807000 \\
\hline $\mathrm{H}$ & 5.36029400 & -1.50141700 & 0.98956700 \\
\hline $\mathrm{C}$ & 4.63524400 & -0.55603900 & 4.17771000 \\
\hline $\mathrm{H}$ & 2.91277000 & 0.61415900 & 4.75359000 \\
\hline $\mathrm{H}$ & 6.23214300 & -1.69765600 & 3.28285200 \\
\hline $\mathrm{H}$ & 5.02132900 & -0.64515700 & 5.18895200 \\
\hline $\mathrm{C}$ & 3.81330100 & -1.30499500 & -1.21833000 \\
\hline
\end{tabular}

$\begin{array}{lrrr}\mathrm{C} & 4.56901000 & -0.86624500 & -2.31876700 \\ \mathrm{C} & 3.70138500 & -2.69073100 & -0.99600100 \\ \mathrm{C} & 5.19054900 & -1.78145000 & -3.17577100 \\ \mathrm{H} & 4.67310800 & 0.19926600 & -2.50452300 \\ \mathrm{C} & 4.32712800 & -3.60489700 & -1.84479800 \\ \mathrm{H} & 3.12440600 & -3.05604000 & -0.15057400 \\ \mathrm{C} & 5.07183500 & -3.15233900 & -2.94034000 \\ \mathrm{H} & 5.76755600 & -1.42036500 & -4.02261100 \\ \mathrm{H} & 4.22733500 & -4.67033400 & -1.65627700 \\ \mathrm{H} & 5.55320200 & -3.86490400 & -3.60407800\end{array}$

E-i

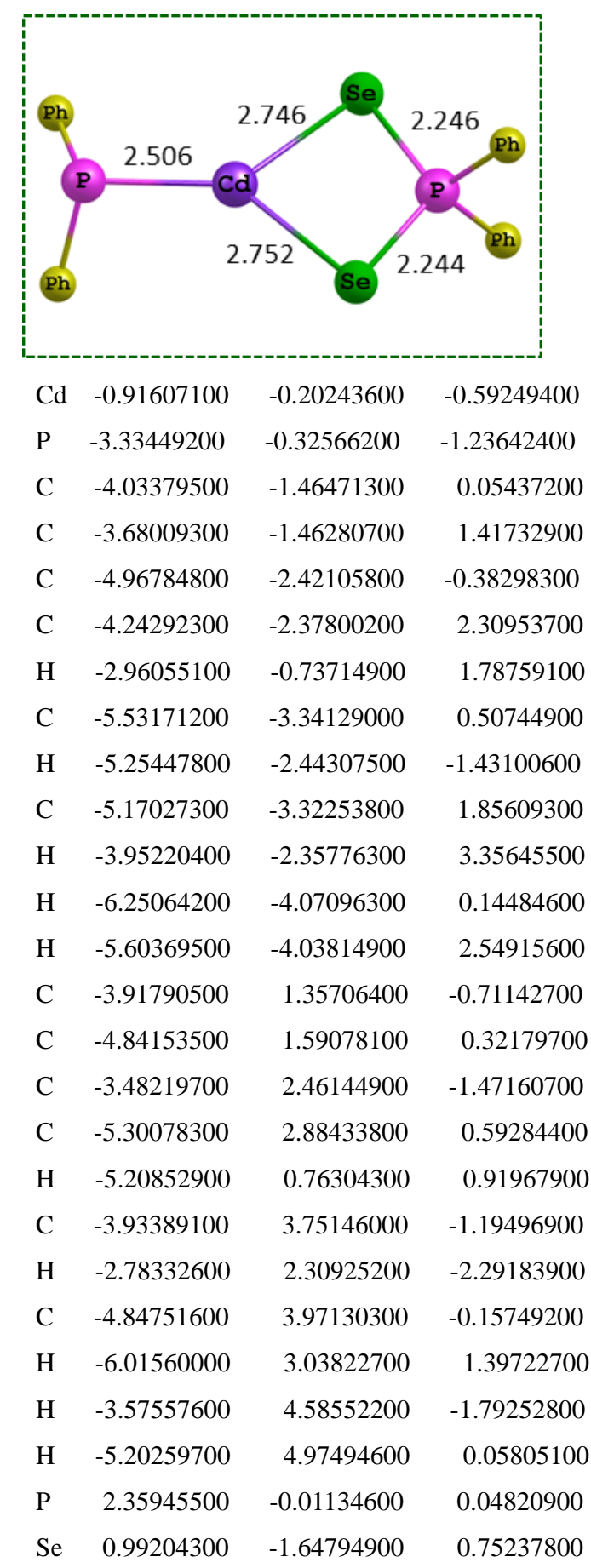




$\begin{array}{lrrr}\mathrm{Se} & 1.19322700 & 1.46712400 & -1.17277000 \\ \mathrm{C} & 3.74928700 & -0.71721500 & -0.91889100 \\ \mathrm{C} & 4.70473200 & -1.50441900 & -0.25351000 \\ \mathrm{C} & 3.85249200 & -0.52031500 & -2.30143000 \\ \mathrm{C} & 5.75515000 & -2.07962000 & -0.96779600 \\ \mathrm{H} & 4.63182200 & -1.66732200 & 0.81788200 \\ \mathrm{C} & 4.90701400 & -1.10128100 & -3.01263600 \\ \mathrm{H} & 3.11414600 & 0.08661700 & -2.81607800 \\ \mathrm{C} & 5.85778500 & -1.87946700 & -2.34918500 \\ \mathrm{H} & 6.49095600 & -2.68558400 & -0.44723900 \\ \mathrm{H} & 4.98237800 & -0.94170100 & -4.08431400 \\ \mathrm{H} & 6.67613700 & -2.32955200 & -2.90382900 \\ \mathrm{C} & 3.16469900 & 0.83029200 & 1.46577900 \\ \mathrm{C} & 4.19387200 & 1.75178100 & 1.20696100 \\ \mathrm{C} & 2.74995100 & 0.60423600 & 2.78384600 \\ \mathrm{C} & 4.80311000 & 2.43207900 & 2.26074000 \\ \mathrm{H} & 4.51943100 & 1.93872000 & 0.18774400 \\ \mathrm{C} & 3.36342000 & 1.29077000 & 3.83640000 \\ \mathrm{H} & 1.95396300 & -0.10626900 & 2.98359100 \\ \mathrm{C} & 4.38820400 & 2.20315700 & 3.57768000 \\ \mathrm{H} & 5.59862000 & 3.14201700 & 2.05400300 \\ \mathrm{H} & 3.03868900 & 1.10819300 & 4.85662600 \\ \mathrm{H} & 4.86341200 & 2.73518600 & 4.39684000\end{array}$

\begin{tabular}{|c|c|c|c|}
\hline $\mathrm{C}$ & 00 & 00 & 00 \\
\hline $\mathrm{C}$ & 4.51716200 & 1.97010400 & -0.31117600 \\
\hline C & 3.09001000 & 1.99816000 & 1.63791700 \\
\hline $\mathrm{C}$ & 5.24286200 & 3.04634600 & 0.20096100 \\
\hline $\mathrm{H}$ & 4.80232300 & 1.54840900 & -1.27168100 \\
\hline $\mathrm{C}$ & 3.81681300 & 3.07941600 & 2.14849300 \\
\hline $\mathrm{H}$ & 2.26027300 & 1.59230900 & 2.2081 \\
\hline$C$ & 4.89478800 & 3.60667200 & $1.43{ }^{2}-2$ \\
\hline $\mathrm{H}$ & 6.08023300 & 7400 & -( \\
\hline $\mathrm{H}$ & 3.53867700 & 3.50414300 & 3.1093 \\
\hline $\mathrm{H}$ & 5.45919500 & 900 & ( \\
\hline $\mathrm{P}$ & -2.98698700 & .35209600 & -1.24944100 \\
\hline $\mathrm{C}$ & -4.22053300 & -1.08853900 & -0.069 \\
\hline$C$ & -4.96564500 & -2.18740200 & -0.53114900 \\
\hline$C$ & -4.43640000 & -0.64631100 & 1.25058700 \\
\hline $\mathrm{C}$ & -5.89159900 & -2.83017900 & 0.29840700 \\
\hline $\mathrm{H}$ & $-4.821^{\prime}$ & -2.538 & -1.5 \\
\hline $\mathrm{C}$ & -5.36570300 & -1.28031700 & 2.07643400 \\
\hline $\mathrm{H}$ & -3.87918700 & 0.20511500 & 500 \\
\hline$C$ & -6.09459700 & -2.37822400 & 1.60351000 \\
\hline $\mathrm{H}$ & -6.45532900 & -3.67856400 & -0.07973800 \\
\hline $\mathrm{H}$ & -5.51625400 & -0.92260200 & 3500 \\
\hline $\mathrm{H}$ & -6.81387100 & -2.87431800 & 2.24890200 \\
\hline $\mathrm{C}$ & -3.27520800 & 1.47719500 & -1.12656400 \\
\hline $\mathrm{C}$ & -4.52742800 & 2.02534800 & -0.79723000 \\
\hline $\mathrm{C}$ & -2.25315200 & 2.35836600 & -1.53075900 \\
\hline $\mathrm{C}$ & -4.74229700 & 3.40617100 & -0.85517600 \\
\hline $\mathrm{H}$ & -5.34278200 & 4075700 & 8900 \\
\hline $\mathrm{C}$ & -2.46807500 & 3.73574500 & -1.58752000 \\
\hline $\mathrm{H}$ & -1.27 & 1.96788700 & 9900 \\
\hline $\mathrm{C}$ & -3.71599200 & 4.26872000 & -1.24682400 \\
\hline $\mathrm{H}$ & -5.71874100 & 3.80496500 & -0.59296200 \\
\hline $\mathrm{H}$ & -1.65 & 4.39237100 & -1.89467300 \\
\hline $\mathrm{H}$ & -3.88437900 & 5.34090200 & -1.28738200 \\
\hline $\mathrm{Cd}$ & -0.91251200 & -0.59881300 & 0.10696100 \\
\hline
\end{tabular}

G-i

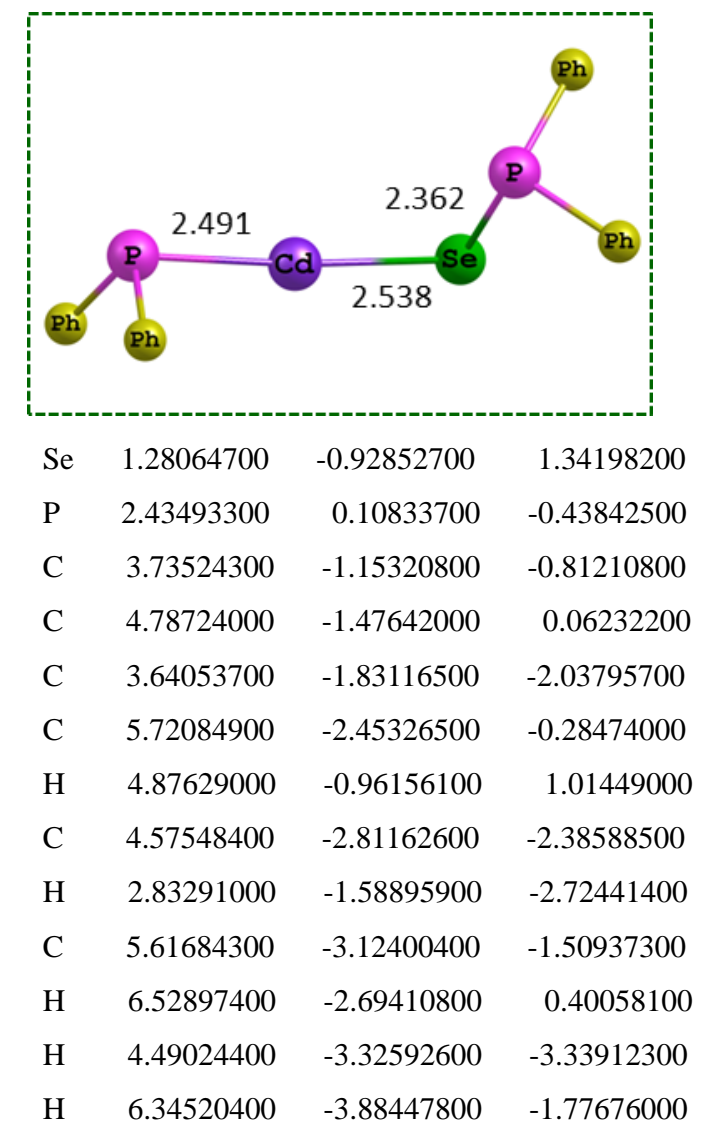

G

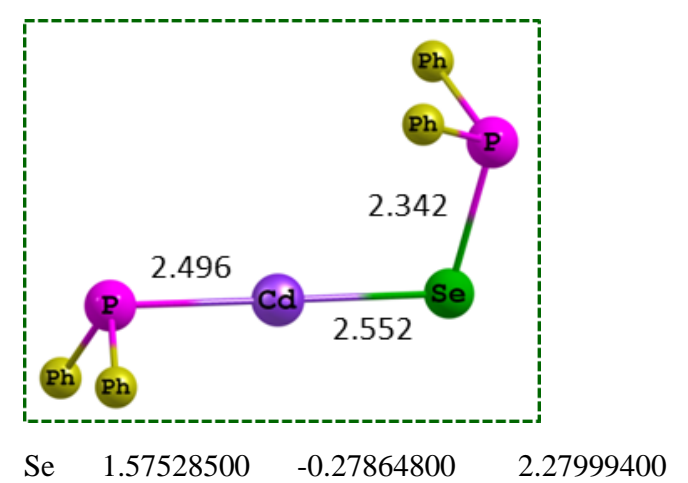




\begin{tabular}{|c|c|c|c|}
\hline & 3.39607400 & 9500 & \\
\hline & 2.60956000 & .74566100 & -0.10870800 \\
\hline & ) & & -1.36882000 \\
\hline & ) & 9200 & 0100 \\
\hline & ) & 0 & 200 \\
\hline & ) & 0 & 6800 \\
\hline & $196-2+2$ & 0 & -0.45 \\
\hline & & & 800 \\
\hline & 400 & 200 & 2200 \\
\hline & 500 & 0 & 100 \\
\hline & 0 & & 0 \\
\hline & 500 & 800 & 0 \\
\hline & 3.84636700 & & 7700 \\
\hline & ) & 0 & 0 \\
\hline & ) & 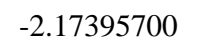 & 00 \\
\hline & 5.29453300 & 300 & 4700 \\
\hline & ) & 0 & 0 \\
\hline & 3.89158300 & -3.16962100 & -0.87 \\
\hline & 2.67555300 & -2.42940500 & 0.74341200 \\
\hline & 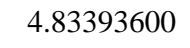 & 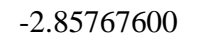 & 00 \\
\hline & 000 & & 3129400 \\
\hline & 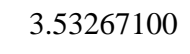 & - & 0 \\
\hline & 21208700 & 2400 & -2.5 \\
\hline & 12803800 & -0.1 & 00 \\
\hline & -3.09686400 & 160788100 & 062613000 \\
\hline & -3.31488800 & -2.67796600 & -1.61509200 \\
\hline & -5.0 & -1.92218400 & 0 \\
\hline & -4.00449000 & -3.85718700 & -1.31037600 \\
\hline & -2.94459800 & 0800 & -2.6 \\
\hline $\mathrm{C}$ & -4.29271300 & 200270000 & 0.96420400 \\
\hline & -3.45 & -1.17486000 & 5800 \\
\hline & -4.49533000 & -4.000 & 200 \\
\hline $\mathrm{H}$ & -4.16021000 & 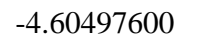 & 0 \\
\hline & -4.67014700 & 0 & 6800 \\
\hline & -5.05130 & -4.90 & .2103030 \\
\hline $\mathrm{C}$ & -315064700 & 1.23284600 & -0.54139600 \\
\hline & -4.54 & & 3200 \\
\hline & 0 & 1 & 0 \\
\hline $\mathrm{C}$ & 00 & 00 & 300 \\
\hline & -5.04919100 & 0.1955 & 6300 \\
\hline & -3.29197400 & 0 & 0 \\
\hline & -1.46753600 & 2.60069200 & -0.53616200 \\
\hline & -4.67587400 & 3.52355800 & - 40000 \\
\hline & -6.37013900 & 2.10070 & 0.000 \\
\hline & -2.79398100 & 4.58241500 & 0.05282600 \\
\hline & 300 & 000 & JI \\
\hline
\end{tabular}

Cd $\quad-0.30143800$

E-i/G-IM1

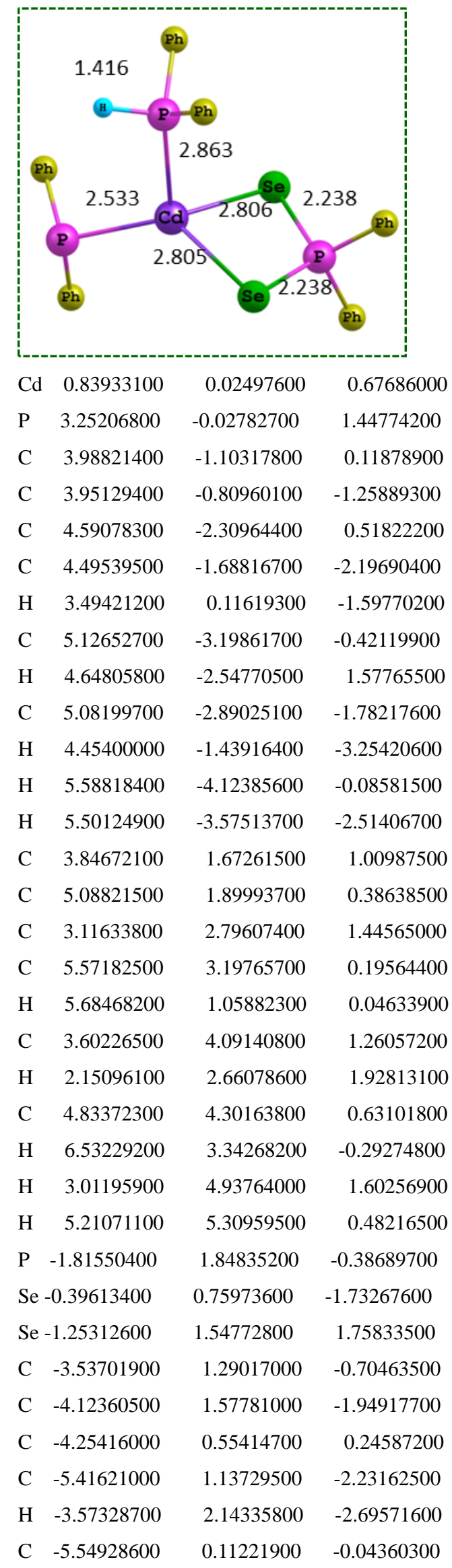




\begin{tabular}{|c|c|c|c|}
\hline I & -3.80078200 & 0.33022700 & 1.20647700 \\
\hline $\mathrm{C}$ & -6.13189800 & 0.40289900 & -1.27867800 \\
\hline & -5.86380100 & 1.36580600 & -3.19460100 \\
\hline $\mathrm{H}$ & -6.09873700 & -0.45811300 & 0.69977100 \\
\hline $\mathrm{H}$ & -7.13840300 & 0.05982500 & -1.50090400 \\
\hline C & -1.83938300 & 3.64228200 & -0.77847100 \\
\hline $\mathrm{C}$ & -2.85180200 & 4.44550400 & -0.22631600 \\
\hline $\mathrm{C}$ & -0.83851900 & 4.22926500 & -1.56247900 \\
\hline $\mathrm{C}$ & -2.86281800 & 5.81943100 & -0.46580300 \\
\hline $\mathrm{H}$ & -3.62876200 & 4.00170800 & 0.38937900 \\
\hline $\mathrm{C}$ & -0.85364200 & 5.60750100 & -1.79788100 \\
\hline $\mathrm{H}$ & -0.05324000 & 3.61065300 & -1.98566000 \\
\hline $\mathrm{C}$ & -1.86300600 & 6.40325600 & -1.25213500 \\
\hline $\mathrm{H}$ & -3.64934500 & 6.43374900 & -0.03704000 \\
\hline $\mathrm{H}$ & -0.07402200 & 6.05481200 & 57000 \\
\hline $\mathrm{H}$ & -1.87246300 & 7.47381800 & -1.43627500 \\
\hline $\mathrm{P}$ & -0.02484800 & -2.69634100 & 0.89191500 \\
\hline$H$ & 0.89427500 & -3.41495400 & 1.69494400 \\
\hline $\mathrm{C}$ & 0.06349400 & -3.72420900 & -0.62435300 \\
\hline $\mathrm{C}$ & -0.53619500 & -3.25549100 & -1.80620200 \\
\hline $\mathrm{C}$ & 0.74286400 & -4.95408600 & -0.64207000 \\
\hline $\mathrm{C}$ & -0.47268700 & -4.01503200 & -2.97670200 \\
\hline $\mathrm{H}$ & -1.03953600 & -2.29278000 & -1.81789300 \\
\hline $\mathrm{C}$ & 0.81370500 & -5.70533700 & -1.81796300 \\
\hline $\mathrm{H}$ & 1.21983800 & -5.32551700 & 0.26058000 \\
\hline$C$ & 0.20231200 & -5.23926100 & -2.98538000 \\
\hline $\mathrm{H}$ & -0.94050100 & -3.64246300 & -3.88341600 \\
\hline $\mathrm{H}$ & 1.34625000 & -6.65213800 & -1.82072900 \\
\hline $\mathrm{H}$ & 0.25773200 & -5.82425800 & -3.89905100 \\
\hline $\mathrm{C}$ & -1.61017500 & -3.15489200 & 1.72453300 \\
\hline $\mathrm{C}$ & -2.40466400 & -4.23570000 & 1.3 \\
\hline $\mathrm{C}$ & -2.02601000 & -2.37727000 & 2.81778200 \\
\hline $\mathrm{C}$ & -3.58625000 & -4.54128900 & 1.99389100 \\
\hline$H$ & -2.10403100 & -4.84008400 & 0.46278400 \\
\hline $\mathrm{C}$ & -3.20084900 & -2.69315300 & 3.50454600 \\
\hline $\mathrm{H}$ & -1.44176000 & -1.51596800 & 3.12881500 \\
\hline$C$ & -3.98416700 & -3.77544000 & 3.09343500 \\
\hline $\mathrm{H}$ & -4.19295800 & -5.38056100 & 1.66530700 \\
\hline H & -3.50666400 & -2.08664100 & 4.35229600 \\
\hline $\mathrm{H}$ & -4.90118000 & -4.01736700 & 3.62326900 \\
\hline
\end{tabular}

E-i/G-TS1

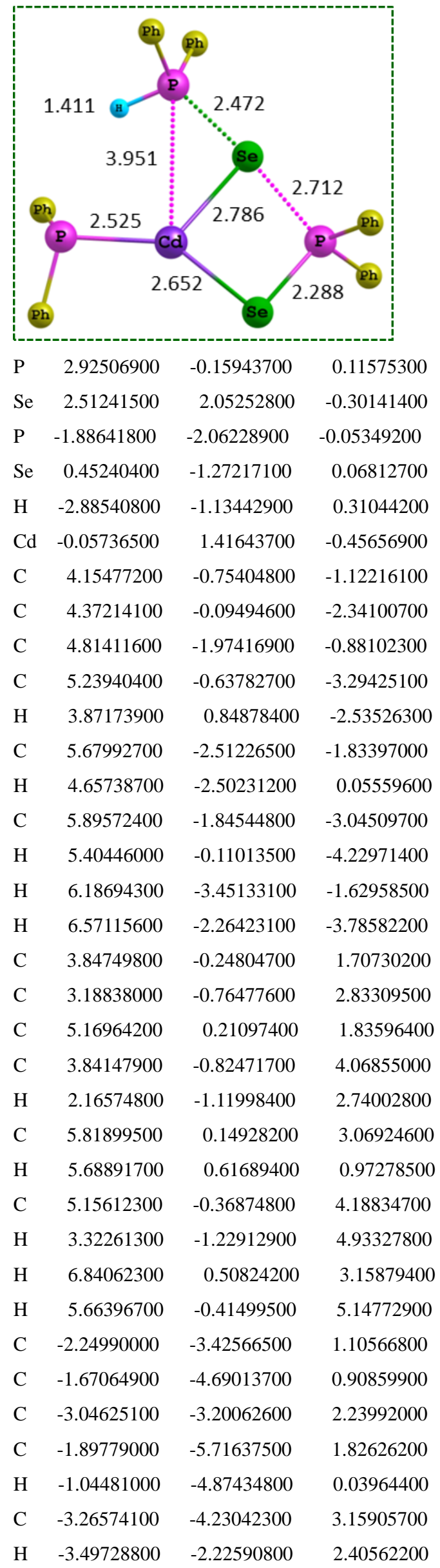




\begin{tabular}{|c|c|c|c|}
\hline $\mathrm{C}$ & 00 & 0 & 2.95386400 \\
\hline $\mathrm{H}$ & 3300 & 39300 & 66314500 \\
\hline & -3.88 & 0 & 100 \\
\hline & -2.86702000 & 0 & 0 \\
\hline & -2.4341260 & .62382600 & 137680 \\
\hline & -1.50 & 0 & 200 \\
\hline $\mathrm{C}$ & 0300 & 6000 & 700 \\
\hline $\mathrm{C}$ & -1.91575500 & 73200 & -4 \\
\hline 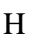 & -0.4 & 00 & 00 \\
\hline $\mathrm{C}$ & -4.17208000 & -3.40627800 & -3.2 \\
\hline $\mathrm{H}$ & & -2.9 & -1.1 \\
\hline $\mathrm{C}$ & -3.24437 & -3.45675 & -4.26393 \\
\hline $\mathrm{H}$ & -1.1 & 0 & 00 \\
\hline$x^{2}$ & -5.206 & 400 & 500 \\
\hline 11 & -3.55973100 & -3.77719200 & 800 \\
\hline$P$ & -2.28500200 & 2.47144700 & 100400500 \\
\hline $\mathrm{C}$ & -3.383028 & 1.77309 & 00 \\
\hline $\mathrm{C}$ & -3 & 1.76210700 & 0 \\
\hline$c$ & -4.59 & 3300 & 600 \\
\hline$C$ & -3.9 & 00 & 300 \\
\hline $\mathrm{H}$ & -2.1 & 2004 & 00 \\
\hline $\mathrm{C}$ & -5.47106300 & 500 & 100 \\
\hline $\mathbf{I}$ & -4.86675 & J & 0 \\
\hline$C_{1}$ & -5.14457300 & 0.61502000 & 00 \\
\hline $\mathrm{H}$ & -3.677 & & 900 \\
\hline 11 & -6.40712000 & 17597600 & 5900 \\
\hline $\mathrm{H}$ & -5.82288900 & 00 & 700 \\
\hline $\mathrm{C}$ & -2.00968500 & 4.22158100 & -0.45379800 \\
\hline $\mathrm{C}$ & -2.880730 & 00570 & 0 \\
\hline $\mathrm{C}$ & -0.953 & 200 & 6000 \\
\hline $\mathrm{C}$ & -2 & 00 & 0 \\
\hline $\mathrm{H}$ & -3.71319800 & 700 & 5100 \\
\hline $\mathrm{C}$ & -0.76760300 & 5100 & -0.75220300 \\
\hline $\mathrm{H}$ & -0.26439300 & 8400 & -1.71550100 \\
\hline $\mathrm{C}$ & -1.63651100 & 400 & 5700 \\
\hline $\mathrm{H}$ & -3.3 & 800 & 9700 \\
\hline $\mathrm{H}$ & 0.06103800 & 6.83899100 & -1.20974500 \\
\hline 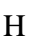 & 1.49060400 & 300 & 600 \\
\hline
\end{tabular}

E-i/G-IM2

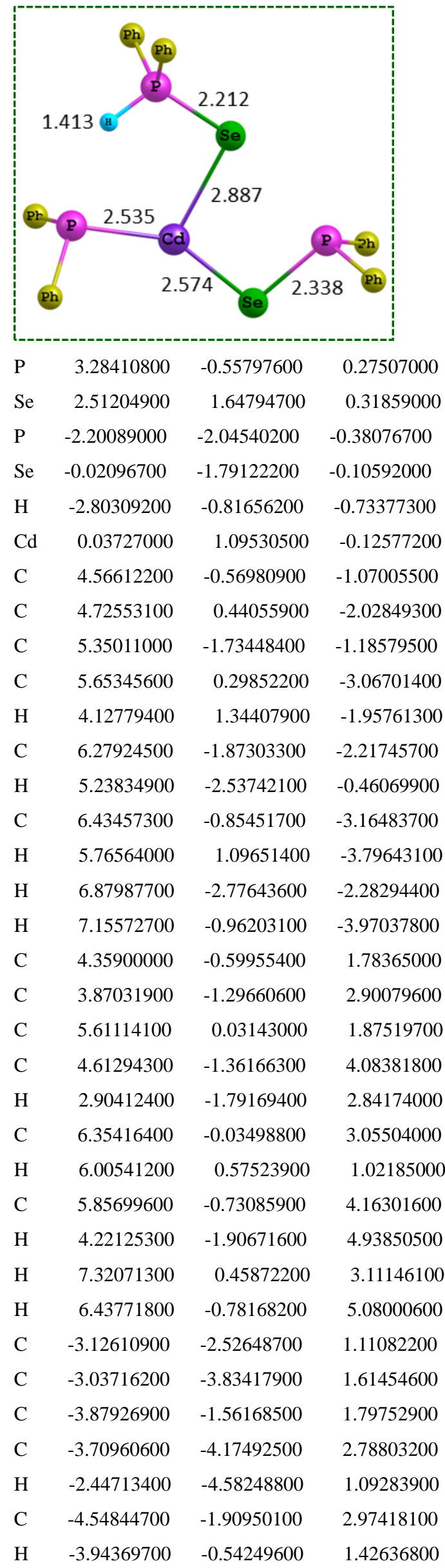




\begin{tabular}{|c|c|c|c|}
\hline & -4.46 & -3.2 & 3.4 \\
\hline & -3.64117100 & -5.18803600 & 3.17316 \\
\hline & -5.12953100 & -1.15893800 & 350140100 \\
\hline & -4.98550000 & -3.48102100 & .38386800 \\
\hline & -2.64525000 & -3.22674100 & -1.70565400 \\
\hline & -1.66652900 & -3.70679300 & -2.58539700 \\
\hline & -3.98566700 & -3.61691500 & -1.86 \\
\hline & -2.02573800 & -4.57417700 & -3.62 \\
\hline & -0.63180700 & -3.40377400 & 68200 \\
\hline & -4.33808400 & -4.47944300 & 2200 \\
\hline & -4.75085800 & -3.25593500 & -1.18780200 \\
\hline & -3.35828300 & -4.95945700 & 1900 \\
\hline & -1.26235200 & -4.94575800 & 24100 \\
\hline & -5.37 & $-4.7^{\prime}$ & -3.0 \\
\hline & -3.63503000 & -5.63274100 & -4.59021400 \\
\hline & -2.32213800 & 1.87176000 & 9100 \\
\hline & -2.98155700 & 2.47222200 & 0.99800400 \\
\hline & -2.20113300 & 2.72655100 & 2.14062600 \\
\hline & -4.377 & 6200 & 29000 \\
\hline & -2.78904800 & 3.12415100 & 3.34631500 \\
\hline & -1.12026200 & 2.62499400 & 2.09214700 \\
\hline & -4.96750700 & 3.04605700 & 2.30668100 \\
\hline & -5.00488600 & 2.44156500 & 0.24175300 \\
\hline & -4.17383700 & 3.28756500 & 3.43277900 \\
\hline & -2.16119700 & 3.31183000 & 4.21307400 \\
\hline & -6.04554100 & 3.17332700 & 2.36093700 \\
\hline & -4.63034300 & 3.60086300 & $4 .$. \\
\hline & -2.03604600 & 3.43335300 & -1.58611800 \\
\hline & -2.09271100 & 4.72448400 & -1.03308300 \\
\hline & -1.7781 & 3.31 & -2.9 \\
\hline & -1.89491500 & 5.85411200 & -1.83267900 \\
\hline & -2.29074800 & 4.85173400 & 0.02663000 \\
\hline & -1.56609500 & 4.44024700 & -3.76015700 \\
\hline & -1.74920300 & 2.32571600 & -3.42323200 \\
\hline & -1.62550200 & 5.71971500 & -3.19729900 \\
\hline & -1.94496900 & 6.84229400 & -1.38258400 \\
\hline & -1.36325400 & 4.31971600 & -4.82111600 \\
\hline & -1.46718100 & 6.59894700 & -3.81518500 \\
\hline
\end{tabular}

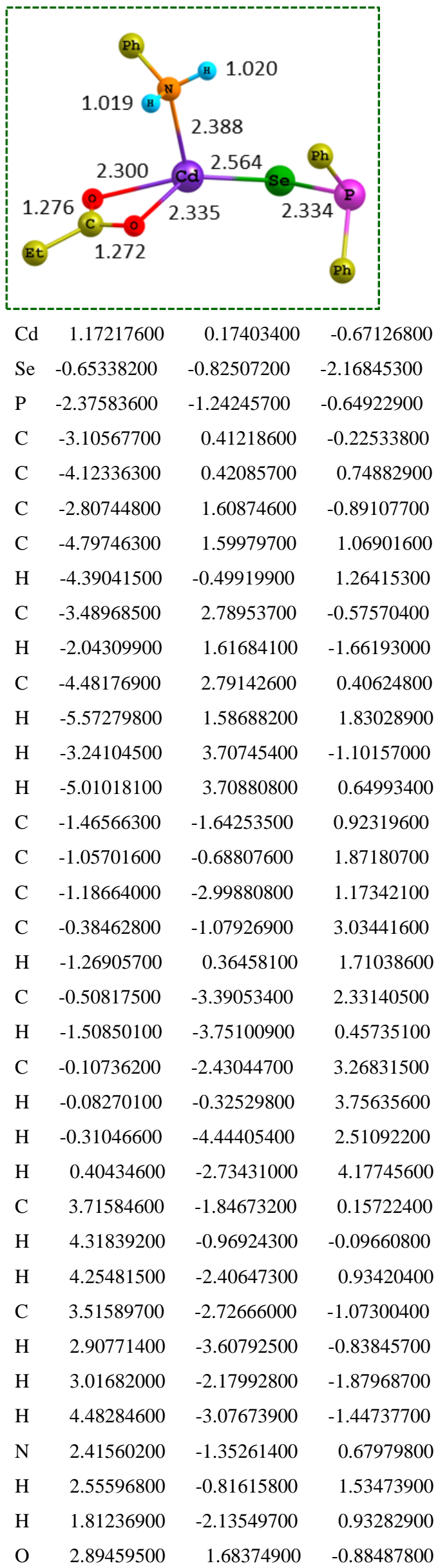




$\begin{array}{llll}\mathrm{O} & 1.58595500 & 1.85117000 & 0.90022800 \\ \mathrm{C} & 2.56145800 & 2.26933300 & 0.19925100 \\ \mathrm{C} & 3.34902800 & 3.49784500 & 0.62667800 \\ \mathrm{H} & 4.41233800 & 3.26410600 & 0.49999000 \\ \mathrm{H} & 3.13178200 & 4.27376600 & -0.11920100 \\ \mathrm{C} & 3.04763300 & 4.00455800 & 2.03790300 \\ \mathrm{H} & 3.63130400 & 4.90635100 & 2.25164000 \\ \mathrm{H} & 1.98677500 & 4.24258900 & 2.15208100 \\ \mathrm{H} & 3.29738500 & 3.25172200 & 2.79231800\end{array}$

$\begin{array}{lrrr}\mathrm{C} & 0.02005400 & 2.09557200 & 2.20073400 \\ \mathrm{H} & 1.82819600 & 0.96903200 & 1.89132200 \\ \mathrm{C} & -1.01816400 & 3.01683300 & 0.21931800 \\ \mathrm{H} & -0.02104200 & 2.61950800 & -1.64119400 \\ \mathrm{C} & -1.00584300 & 2.83647800 & 1.60685800 \\ \mathrm{H} & 0.04086200 & 1.95847000 & 3.27887300 \\ \mathrm{H} & -1.82157500 & 3.57001700 & -0.25655800 \\ \mathrm{H} & -1.78997200 & 3.27196000 & 2.21975700 \\ \mathrm{C} & 3.58566100 & 0.38090400 & 0.09103500 \\ \mathrm{C} & 4.49458300 & 1.30874400 & 0.63454600 \\ \mathrm{C} & 3.71882400 & -0.97041800 & 0.43891600 \\ \mathrm{C} & 5.48366300 & 0.90023100 & 1.53134600 \\ \mathrm{H} & 4.42961900 & 2.35803800 & 0.35473000 \\ \mathrm{C} & 4.71736100 & -1.38200200 & 1.32844300 \\ \mathrm{H} & 3.05227600 & -1.70581600 & -0.00108400 \\ \mathrm{C} & 5.59770600 & -0.44925800 & 1.88171600 \\ \mathrm{H} & 6.17105700 & 1.63252400 & 1.94570400 \\ \mathrm{H} & 4.80808800 & -2.43457100 & 1.58316100 \\ \mathrm{H} & 6.37404200 & -0.77081800 & 2.57019900\end{array}$

\section{C/H-TS1}

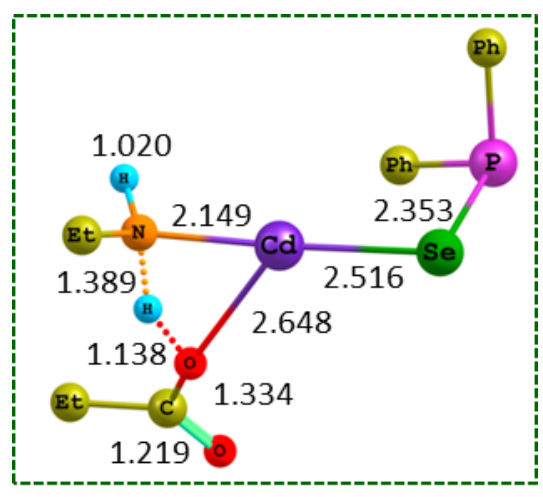

$\begin{array}{lrrr}\mathrm{Cd} & -0.51921900 & -1.06459600 & -0.39429600 \\ \mathrm{C} & -2.32716900 & -3.03501000 & 1.28156400 \\ \mathrm{H} & -2.63097100 & -3.43327300 & 0.30562000 \\ \mathrm{H} & -1.46288100 & -3.63076600 & 1.60952800 \\ \mathrm{C} & -3.47233500 & -3.21233900 & 2.28243700 \\ \mathrm{H} & -3.18918700 & -2.83803400 & 3.27382200 \\ \mathrm{H} & -4.36540300 & -2.66872000 & 1.95706700 \\ \mathrm{H} & -3.73379100 & -4.27141500 & 2.38835800 \\ \mathrm{O} & -3.96468900 & 1.69146000 & -1.39854200 \\ \mathrm{C} & -3.95577000 & 0.78167800 & -0.58736600 \\ \mathrm{C} & -5.04789600 & 0.59961800 & 0.46780800 \\ \mathrm{H} & -5.51550100 & -0.37788100 & 0.28810400 \\ \mathrm{H} & -4.56258000 & 0.51905500 & 1.45007700 \\ \mathrm{C} & -6.09944300 & 1.70886900 & 0.46807600 \\ \mathrm{H} & -6.86003000 & 1.51652700 & 1.23239400 \\ \mathrm{H} & -5.64449000 & 2.68285000 & 0.67062200 \\ \mathrm{H} & -6.59363500 & 1.77829700 & -0.50462100 \\ \mathrm{~N} & -1.96748900 & -1.61449300 & 1.09541200 \\ \mathrm{H} & -1.72487000 & -1.20400600 & 1.99717000 \\ \mathrm{O} & -2.99700300 & -0.14520400 & -0.56457700 \\ \mathrm{H} & -2.81252600 & -0.81313200 & 0.33839000 \\ \mathrm{P} & 2.36237100 & 1.07721800 & -1.11986100 \\ \mathrm{Se} & 1.30712400 & -0.78299900 & -2.10203500 \\ \mathrm{C} & 1.03386300 & 1.71188600 & 0.01792300 \\ \mathrm{C} & 1.03239000 & 1.53275200 & 1.41473000 \\ \mathrm{C} & -0.00165300 & 2.47010600 & -0.56492300\end{array}$

\section{C/H-IM2}

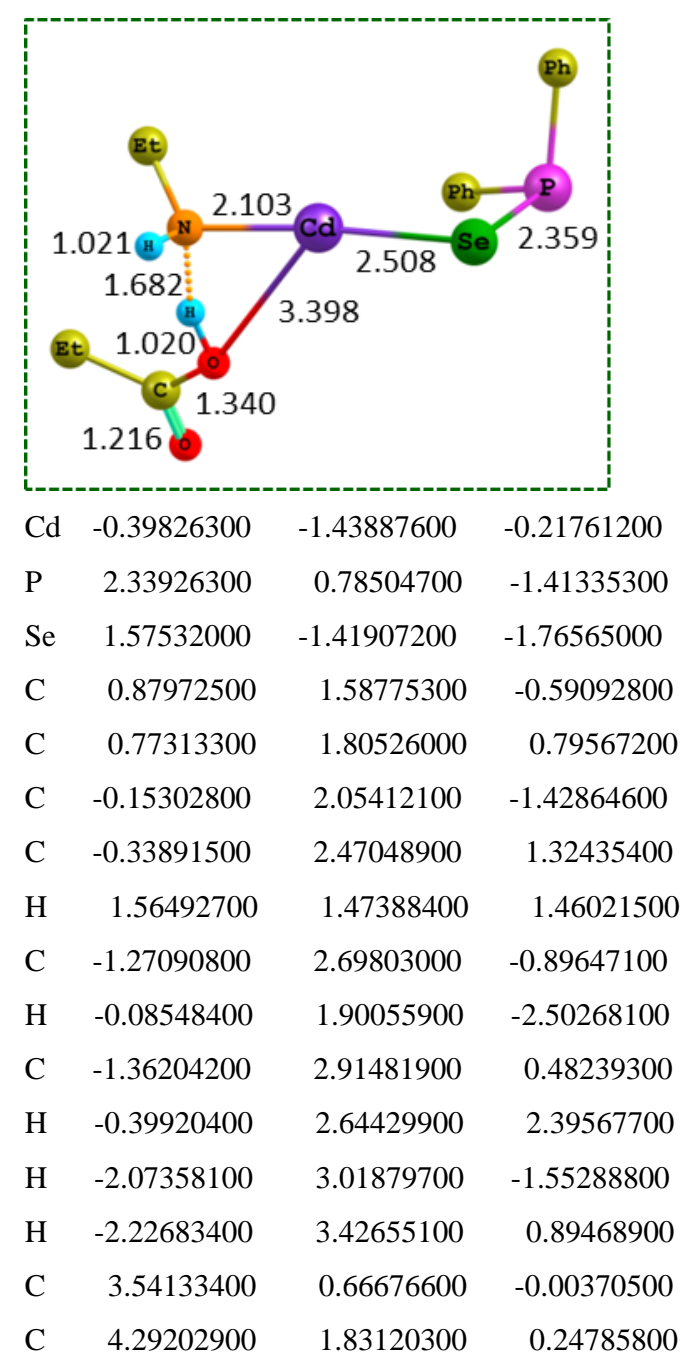




$\begin{array}{lrrr}\mathrm{C} & 3.80955200 & -0.48092000 & 0.75469400 \\ \mathrm{C} & 5.25665000 & 1.85617800 & 1.25664800 \\ \mathrm{H} & 4.12165800 & 2.72517700 & -0.34821500 \\ \mathrm{C} & 4.78541800 & -0.45974600 & 1.75734900 \\ \mathrm{H} & 3.26583700 & -1.39805500 & 0.55050800 \\ \mathrm{C} & 5.50664000 & 0.70807200 & 2.01581400 \\ \mathrm{H} & 5.82049600 & 2.76654200 & 1.44028100 \\ \mathrm{H} & 4.98281700 & -1.36042800 & 2.33250400 \\ \mathrm{H} & 6.26526900 & 0.72196700 & 2.79319600 \\ \mathrm{C} & -2.07020500 & -1.20803600 & 2.36067300 \\ \mathrm{H} & -1.80688900 & -0.14462900 & 2.40563600 \\ \mathrm{H} & -3.07714900 & -1.29606500 & 2.79675600 \\ \mathrm{C} & -1.09118600 & -2.01547300 & 3.21983100 \\ \mathrm{H} & -1.33528000 & -3.08418100 & 3.19689100 \\ \mathrm{H} & -0.06005700 & -1.89820200 & 2.86166200 \\ \mathrm{H} & -1.12055800 & -1.68611500 & 4.26552100 \\ \mathrm{O} & -3.43136900 & -0.02172300 & -0.79714700 \\ \mathrm{H} & -2.14791500 & -1.59452200 & 0.93856600 \\ \mathrm{H} & -4.99536500 & 1.45564800 & -1.36818800 \\ \mathrm{C} & -4.58689100 & 0.62152700 & -0.58283900 \\ \mathrm{C} & -5.33644200 & 0.23355300 & 0.69129000 \\ \mathrm{H} & -5.49622200 & -0.85280900 & 0.66960100 \\ \mathrm{H} & -4.66870500 & 0.41842500 & 1.54348000 \\ \mathrm{H} & -6.66172900 & 0.97387100 & 0.86998700 \\ \mathrm{H} & -7.15385500 & 0.65933100 & 1.79636100 \\ \mathrm{H} & -7.33677200 & 0.77461300 & 0.03330800 \\ \mathrm{H} & -2.55704100 & 0.88193400\end{array}$

\begin{tabular}{|c|c|c|c|}
\hline $\mathrm{C}$ & .0 & 0 & \\
\hline $\mathrm{H}$ & 24846600 & 65472600 & 187021700 \\
\hline & 3.48446100 & 13551000 & -0.69 \\
\hline & 4.82651600 & 0 & 30 \\
\hline & 48248600 & 04180000 & -1.66097400 \\
\hline & 0 & & 400 \\
\hline & 3.83682900 & 10939600 & 6207300 \\
\hline $\mathrm{H}$ & 2.051 & 300 & 0600 \\
\hline C & 10 & 00 & 800 \\
\hline $\mathrm{C}$ & 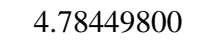 & 0 & 0 \\
\hline 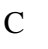 & 3.65 & & 800 \\
\hline $\mathrm{C}$ & 100 & -1.80786500 & 0 \\
\hline $\mathrm{H}$ & 0 & 0 & 9500 \\
\hline$C$ & 3.66 & -3.3 & 00 \\
\hline $\mathrm{H}$ & 3.22130300 & -2.54816600 & 0.17670200 \\
\hline$C$ & 4.22738900 & -3.06373500 & -3.03558800 \\
\hline $\mathrm{H}$ & 307900 & -1.59457 & -4.25933 \\
\hline $\mathrm{H}$ & 32 & 0 & 00 \\
\hline te & 4.23142600 & 3122100 & 3500 \\
\hline & 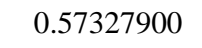 & 0 & \\
\hline $\mathrm{Se}$ & -1.12143100 & 050556400 & 5640400 \\
\hline in & -0.77261400 & -0.3 & 300 \\
\hline $\mathrm{H}$ & -( & 0.61819300 & 0 \\
\hline $\mathrm{H}$ & -1.73590300 & 0.3658 & 400 \\
\hline $\mathrm{C}$ & -( & 0 & \\
\hline 11 & 0.56099600 & -1.29011600 & 4.260 \\
\hline $\mathrm{H}$ & -0.64078600 & -2.32 & 200 \\
\hline $\mathrm{C}$ & -1.37430900 & -1.26621400 & 5.25252800 \\
\hline $\mathrm{H}$ & -2.43727000 & -1.35326500 & $5 .($ \\
\hline П & -1.12623400 & -2.0 & 0 \\
\hline $\mathrm{H}$ & 122382 & 0 & 00 \\
\hline$P$ & -3.0632 & -0.2 & 00 \\
\hline $\mathrm{C}$ & -4.27147800 & -1.19588800 & -0.69959800 \\
\hline$r$ & -4.81489500 & 0.73077100 & 10080 \\
\hline $\mathrm{C}$ & -4.64568900 & -2.46686300 & 00 \\
\hline $\mathrm{C}$ & -5.71 & -1 & -2.63125400 \\
\hline $\mathrm{H}$ & -4.53 & 0 & 00 \\
\hline $\mathrm{C}$ & -5.54176800 & -3.25790600 & -0.95774300 \\
\hline$H$ & -4.23372300 & -2.83878300 & 0 \\
\hline $\mathrm{C}$ & -6.07685900 & -2.78492400 & -2.15872100 \\
\hline $\mathrm{H}$ & -6.12 & -1.1 & -3 \\
\hline $\mathrm{H}$ & -5.82 & 00 & -0 . \\
\hline $\mathrm{H}$ & -6.77487700 & -3.39680200 & -2 \\
\hline $\mathrm{C}$ & -3.61745000 & 1.51224300 & -0.0100340 \\
\hline $\mathrm{C}$ & -4.87866700 & 37400 & 0.49817400 \\
\hline $\mathrm{C}$ & -2.82723500 & 2.49652600 & -0.61910800 \\
\hline
\end{tabular}




$\begin{array}{lrrr}\mathrm{C} & -5.34213800 & 3.19116200 & 0.38067400 \\ \mathrm{H} & -5.50733600 & 1.13599400 & 0.98178200 \\ \mathrm{C} & -3.28935300 & 3.81348100 & -0.72896900 \\ \mathrm{H} & -1.85315100 & 2.22981800 & -1.01777500 \\ \mathrm{C} & -4.54606900 & 4.16521500 & -0.23318700 \\ \mathrm{H} & -6.32211100 & 3.45269200 & 0.77065200 \\ \mathrm{H} & -2.66365300 & 4.56173700 & -1.20818400 \\ \mathrm{H} & -4.90384100 & 5.18718000 & -0.32126700\end{array}$

\section{E/H-TS1}

\begin{tabular}{|c|c|c|}
\hline & 1. & 2.330 \\
\hline & & \\
\hline 2.93876000 & -2.23020500 & 0.1477240 \\
\hline 3.90267000 & -0.84536500 & -1.45958100 \\
\hline 3.47586100 & 0.85353000 & -0.83840000 \\
\hline 2.27744200 & 1.53248300 & 800 \\
\hline 4.42184100 & 1.48255900 & -0.01063100 \\
\hline 2.02928500 & 2.79821400 & -0.58 \\
\hline 1.53852100 & 1.07837900 & -1.77 \\
\hline 4.17213800 & 2.74502800 & 0.53405700 \\
\hline 5.357 & 9000 & 900 \\
\hline 2.97380800 & 3.40693300 & 0.24981100 \\
\hline 1.09911600 & 9700 & 900 \\
\hline 4.91509400 & 3.21290200 & 4400 \\
\hline 2.78038400 & 4.39131600 & 0.66702500 \\
\hline 2.82562400 & 0.96819100 & -2.96813100 \\
\hline 3.08568200 & -0.06925600 & -4.02110100 \\
\hline 1.88477500 & -1.98663900 & -3.17750600 \\
\hline 2.40232500 & -0.16939000 & -5.23380700 \\
\hline 3.82388800 & 0.71904700 & -3.89238000 \\
\hline 1.20483700 & -2.09126900 & -4.39642800 \\
\hline 1.68 & -2.6999 & -2.38376300 \\
\hline 1.45674800 & -1.18314400 & -5.42631800 \\
\hline 2.61175600 & 0.54072200 & -6.02947100 \\
\hline 0.4 & -2.88 & -4.53485100 \\
\hline 0.92700500 & -1.26476000 & -6.37135400 \\
\hline $\mathrm{Cd} \quad 0.78121300$ & -0.94716800 & 0.75376000 \\
\hline Se -1.28587500 & -0.97065400 & -1.17416600 \\
\hline $\mathrm{N} \quad-0.30810700$ & -0.01834100 & 2.37522500 \\
\hline-0.05225500 & 0.97004900 & 2.40 \\
\hline
\end{tabular}

$$
\begin{array}{lrrr}
\mathrm{H} & -1.90774400 & -0.06388100 & 1.55129700 \\
\mathrm{C} & -0.15702400 & -0.58023300 & 3.72551200 \\
\mathrm{H} & 0.89256900 & -0.60214200 & 4.06515300 \\
\mathrm{H} & -0.48335000 & -1.62998300 & 3.69755500 \\
\mathrm{C} & -0.98995000 & 0.17468300 & 4.76932000 \\
\mathrm{H} & -2.05408900 & 0.16551900 & 4.50600600 \\
\mathrm{H} & -0.87587000 & -0.27122900 & 5.76516000 \\
\mathrm{H} & -0.67202500 & 1.22262000 & 4.83485800 \\
\mathrm{P} & -2.73821700 & -0.31202000 & 0.36904500 \\
\mathrm{C} & -3.96142100 & -1.58091400 & 0.82953000 \\
\mathrm{C} & -4.99277200 & -1.95339200 & -0.04766000 \\
\mathrm{C} & -3.83837000 & -2.22447900 & 2.07076400 \\
\mathrm{C} & -5.89415100 & -2.95268300 & 0.31908500 \\
\mathrm{H} & -5.08937700 & -1.46626900 & -1.01384000 \\
\mathrm{C} & -4.74344400 & -3.22615200 & 2.43308900 \\
\mathrm{H} & -3.03939300 & -1.94701800 & 2.75258800 \\
\mathrm{C} & -5.77064100 & -3.59011600 & 1.55912300 \\
\mathrm{H} & -6.69008600 & -3.23687000 & -0.36303700 \\
\mathrm{H} & -4.64415800 & -3.71970700 & 3.39545500 \\
\mathrm{H} & -6.47279300 & -4.36944300 & 1.84071800 \\
\mathrm{C} & -3.68248400 & 1.17830800 & -0.11857800 \\
\mathrm{C} & -4.71184100 & 1.64095800 & 0.72045400 \\
\mathrm{C} & -3.35873600 & 1.89550700 & -1.27788800 \\
\mathrm{C} & -5.40421400 & 2.80910300 & 0.39957600 \\
\mathrm{H} & -4.97756200 & 1.08986300 & 1.61850500 \\
\mathrm{H} & -4.05867000 & 3.06313200 & -1.59638500 \\
\mathrm{H} & -2.56650800 & 1.53518200 & -1.92748700 \\
\hline
\end{array}
$$

E/H-IM2

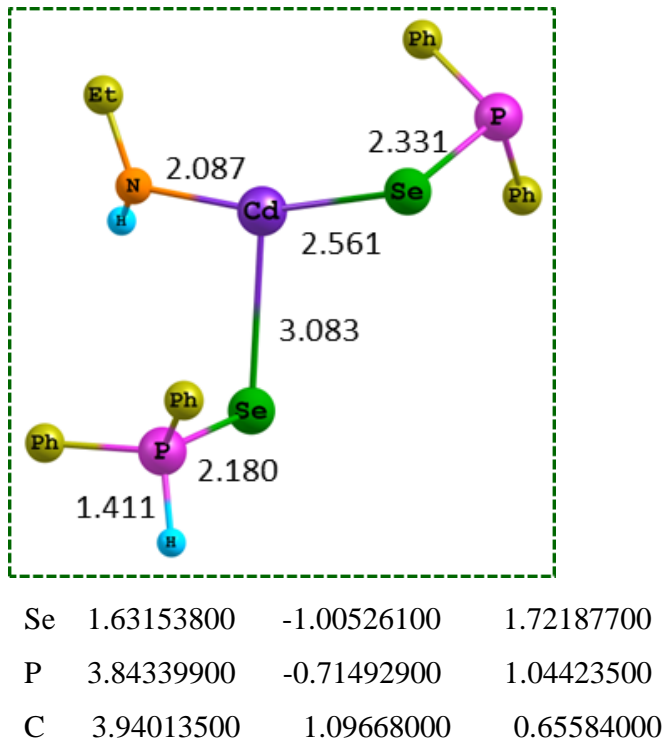




\begin{tabular}{|c|c|c|c|}
\hline $\mathrm{C}$ & 3.66534600 & 1.66004700 & -0.60259200 \\
\hline $\mathrm{C}$ & 4.32610400 & 1.95084800 & 1.70390600 \\
\hline$C$ & 3.76861000 & 3.03957700 & -0.80329300 \\
\hline $\mathrm{H}$ & 3.38507800 & 1.01966500 & -1.43395100 \\
\hline $\mathrm{C}$ & 4.42254700 & 3.33031000 & 1.50569100 \\
\hline $\mathrm{H}$ & 4.54991800 & 1.53016500 & 2.68109500 \\
\hline $\mathrm{C}$ & 4.14522900 & 3.87883100 & 0.24988500 \\
\hline $\mathrm{H}$ & 3.55636500 & 3.45718900 & -1.78392500 \\
\hline $\mathrm{H}$ & 4.72184300 & 3.97404700 & 2.32834300 \\
\hline . & 4.22569600 & 4.95061900 & 0.09163100 \\
\hline $\mathrm{C}$ & 3.96910400 & -1.45590500 & -0.65461300 \\
\hline $\mathrm{C}$ & 5.22002100 & -1.34788800 & -1.29385100 \\
\hline$C$ & 2.96373500 & -2.20755100 & -1.27877900 \\
\hline $\mathrm{C}$ & 5.44542200 & -1.94692100 & -2.53375900 \\
\hline $\mathrm{H}$ & 6.02397900 & -0.78790700 & 5300 \\
\hline $\mathrm{C}$ & 3.19239400 & -2.81531000 & -2.51922700 \\
\hline $\mathrm{H}$ & 2.00066400 & -2.32582300 & -0.79175800 \\
\hline $\mathrm{C}$ & 4.42953000 & -2.68455800 & -3.15266900 \\
\hline $\mathrm{H}$ & 6.41513900 & -1.84345200 & -3.01326600 \\
\hline $\mathrm{H}$ & 2.39794400 & -3.39106100 & -2.98653300 \\
\hline $\mathrm{H}$ & 4.60525400 & -3.15650400 & -4.11533200 \\
\hline $\mathrm{Cd}$ & 0.27329600 & 0.54325700 & 0.20072900 \\
\hline $\mathrm{Se}$ & $-1.323 t$ & -1.38554900 & -1.59694700 \\
\hline $\mathrm{C}$ & -0.26756800 & 3.58424500 & -0.05805100 \\
\hline $\mathrm{H}$ & 0.70144800 & 3.84763100 & -0.51561000 \\
\hline $\mathrm{H}$ & -0.10250100 & 3.59584600 & 1.02706100 \\
\hline $\mathrm{C}$ & -1.28509900 & 4.67709600 & -0.41321600 \\
\hline $\mathrm{H}$ & -2.24122700 & 4.49423800 & 0.09009200 \\
\hline $\mathrm{H}$ & -0.91981900 & 5.66807400 & -0.11495000 \\
\hline $\mathrm{H}$ & -1.46991100 & 4.70166600 & -1.49479900 \\
\hline $\mathrm{P}$ & -3.26270000 & -1.34731500 & -0.60104500 \\
\hline $\mathrm{C}$ & -4.41132000 & -0.01221200 & -1.05941700 \\
\hline $\mathrm{C}$ & -4.04027400 & 1.33286400 & -0.89733000 \\
\hline $\mathrm{C}$ & -5.66707100 & -0.33921200 & -1.59511400 \\
\hline $\mathrm{C}$ & -4.93923900 & 2.33797000 & -1.25879100 \\
\hline $\mathrm{H}$ & -3.05 & 400 & -0.5 \\
\hline $\mathrm{C}$ & -6.55773200 & 0.67523100 & -1.95566200 \\
\hline $\mathrm{H}$ & -5.95330900 & -1.37866600 & -1.73401000 \\
\hline $\mathrm{C}$ & -6.19451000 & 2.01388000 & -1.78513800 \\
\hline $\mathrm{H}$ & -4.65162000 & 3.37787900 & -1.13407700 \\
\hline $\mathrm{H}$ & -7.52789700 & 0.41886800 & -2.37104100 \\
\hline $\mathrm{H}$ & -6.88569100 & 2.80300400 & -2.06741800 \\
\hline $\mathrm{C}$ & -3.19084800 & -1.42369200 & 1.22790100 \\
\hline $\mathrm{C}$ & -4.21624900 & -0.86485300 & 2.00823300 \\
\hline $\mathrm{C}$ & -2.11694100 & -2.07782600 & 1.85061100 \\
\hline $\mathrm{C}$ & -4.16516600 & -0.96691800 & 3.40018900 \\
\hline
\end{tabular}

$\begin{array}{lrrr}\mathrm{H} & -5.04432300 & -0.34426200 & 1.53797900 \\ \mathrm{C} & -2.07182600 & -2.17367900 & 3.24341300 \\ \mathrm{H} & -1.31119100 & -2.49433300 & 1.25354800 \\ \mathrm{C} & -3.09440700 & -1.61971000 & 4.01830400 \\ \mathrm{H} & -4.95700600 & -0.52743900 & 3.99971600 \\ \mathrm{H} & -1.22996600 & -2.66937700 & 3.71673400 \\ \mathrm{H} & -3.05317100 & -1.68908900 & 5.10145900 \\ \mathrm{H} & -4.00633000 & -2.48572900 & -0.97797300 \\ \mathrm{~N} & -0.74650600 & 2.24879200 & -0.43657000 \\ \mathrm{H} & -0.86525300 & 2.20713400 & -1.44935300\end{array}$

F/I-i-IM1

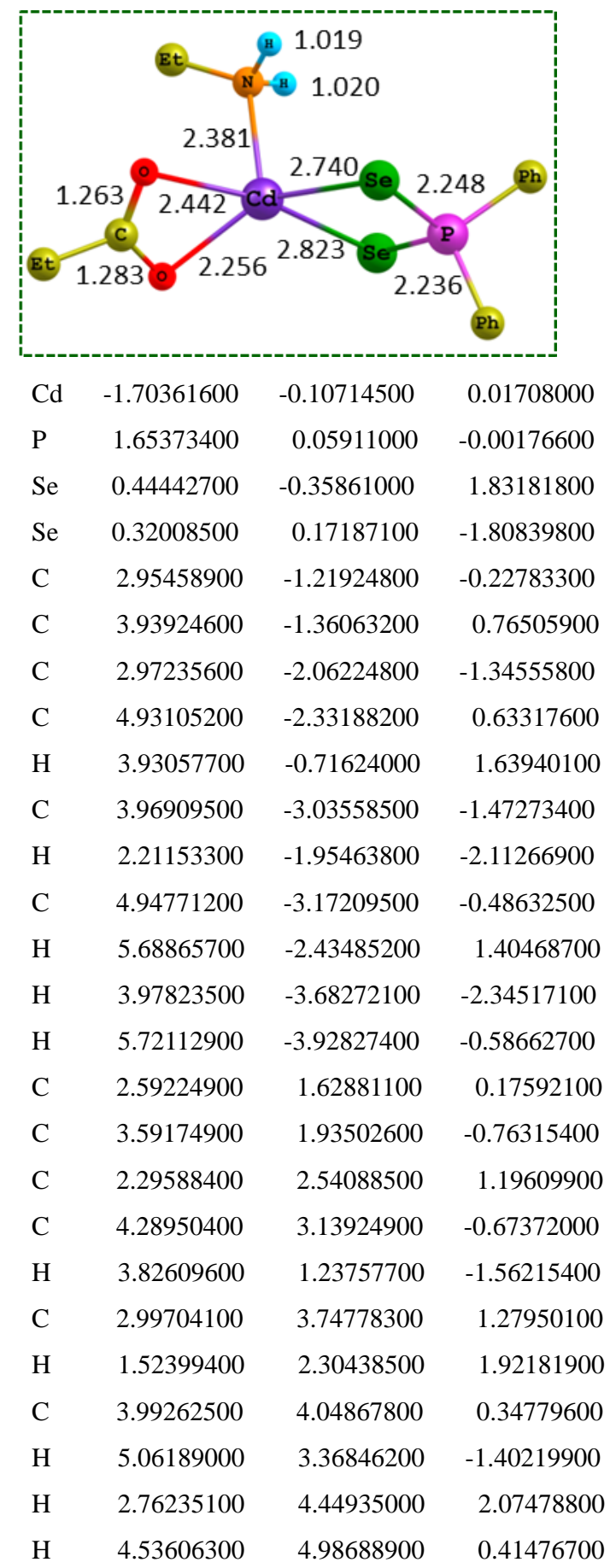




$\begin{array}{lrrr}\mathrm{C} & -3.67935000 & -2.80025100 & 0.31089900 \\ \mathrm{H} & -4.29883900 & -2.26829600 & -0.41611100 \\ \mathrm{H} & -3.82275600 & -3.87890500 & 0.15782300 \\ \mathrm{C} & -4.08100400 & -2.41626600 & 1.73192500 \\ \mathrm{H} & -3.45904200 & -2.92920600 & 2.47549400 \\ \mathrm{H} & -3.98975800 & -1.33733100 & 1.89338000 \\ \mathrm{H} & -5.12175500 & -2.69997300 & 1.91763000 \\ \mathrm{O} & -3.26483500 & 1.31681100 & 0.80709900 \\ \mathrm{O} & -3.83954300 & 0.31201600 & -1.08898700 \\ \mathrm{C} & -4.08613400 & 1.13240300 & -0.16079700 \\ \mathrm{C} & -5.37185300 & 1.94724300 & -0.15175700 \\ \mathrm{H} & -5.84159500 & 1.79713000 & 0.82798200 \\ \mathrm{H} & -5.07209900 & 3.00269400 & -0.16942200 \\ \mathrm{C} & -6.34437500 & 1.63496400 & -1.28960300 \\ \mathrm{H} & -7.23131300 & 2.27434300 & -1.22034100 \\ \mathrm{H} & -5.87553600 & 1.79821300 & -2.26400800 \\ \mathrm{H} & -6.67163100 & 0.59125500 & -1.25661800 \\ \mathrm{~N} & -2.26912200 & -2.41957800 & 0.03548300 \\ \mathrm{H} & -1.98910900 & -2.74424300 & -0.88898100 \\ \mathrm{H} & -1.63636700 & -2.86477400 & 0.70027700\end{array}$

F/I-i-TS1

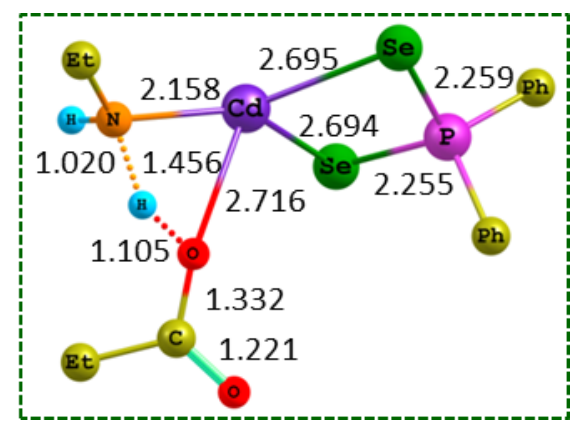

$\begin{array}{rrrc}\mathrm{Cd} & -1.24420800 & -1.32960100 & 0.11215600 \\ \mathrm{C} & -3.94962500 & -2.59341600 & -0.93058800 \\ \mathrm{H} & -3.63998100 & -2.18811800 & -1.90181400 \\ \mathrm{H} & -3.56293200 & -3.62262700 & -0.88333500 \\ \mathrm{C} & -5.47936800 & -2.62988100 & -0.85904100 \\ \mathrm{H} & -5.81795200 & -3.04632000 & 0.09778400 \\ \mathrm{H} & -5.90089400 & -1.62385000 & -0.95815800 \\ \mathrm{H} & -5.89173700 & -3.25566200 & -1.65921200 \\ \mathrm{O} & -2.99259600 & 2.88178100 & 0.17776000 \\ \mathrm{C} & -3.58938100 & 1.81670200 & 0.18489000 \\ \mathrm{C} & -5.03148500 & 1.67519300 & 0.66823600 \\ \mathrm{H} & -5.62552500 & 1.30434700 & -0.17787800 \\ \mathrm{H} & -5.05987100 & 0.87586200 & 1.42092200 \\ \mathrm{C} & -5.62671600 & 2.96883300 & 1.22357100 \\ \mathrm{H} & -6.66112200 & 2.80716500 & 1.54502500 \\ \mathrm{H} & -5.05033200 & 3.32997600 & 2.08007500\end{array}$

\begin{tabular}{lrrr}
$\mathrm{H}$ & -5.61710000 & 3.75928300 & 0.46826900 \\
$\mathrm{~N}$ & -3.36251200 & -1.73921400 & 0.12016900 \\
$\mathrm{H}$ & -3.67040000 & -2.07422600 & 1.03297700 \\
$\mathrm{O}$ & -3.01872600 & 0.69401100 & -0.24896100 \\
$\mathrm{H}$ & -3.46681600 & -0.29836600 & -0.05936900 \\
$\mathrm{P}$ & 1.69821800 & 0.00339100 & -0.00821800 \\
$\mathrm{Se}$ & 0.53594800 & -0.36451100 & 1.88900200 \\
$\mathrm{Se}$ & 0.79996000 & -1.27053300 & -1.64332600 \\
$\mathrm{C}$ & 3.46199600 & -0.40178800 & 0.26270000 \\
$\mathrm{C}$ & 4.08882400 & -1.44387700 & -0.43179700 \\
$\mathrm{C}$ & 4.18004200 & 0.32463900 & 1.22887700 \\
$\mathrm{C}$ & 5.42872900 & -1.74828900 & -0.17232300 \\
$\mathrm{H}$ & 3.53191900 & -2.01693400 & -1.16651100 \\
$\mathrm{C}$ & 5.51531400 & 0.01449600 & 1.48442000 \\
$\mathrm{H}$ & 3.69983300 & 1.12761100 & 1.78065300 \\
$\mathrm{C}$ & 6.14217500 & -1.02203700 & 0.78334300 \\
$\mathrm{H}$ & 5.90977800 & -2.55532400 & -0.71709500 \\
$\mathrm{H}$ & 6.06436800 & 0.57953600 & 2.23189600 \\
$\mathrm{H}$ & 7.18200700 & -1.26253200 & 0.98516800 \\
$\mathrm{C}$ & 1.63223200 & 1.76816900 & -0.48639600 \\
$\mathrm{C}$ & 2.73610000 & 2.37960000 & -1.10451400 \\
$\mathrm{C}$ & 0.44106000 & 2.49014800 & -0.31242400 \\
$\mathrm{C}$ & 2.64689600 & 3.70615900 & -1.53171700 \\
$\mathrm{H}$ & 3.66009700 & 1.83060900 & -1.25407300 \\
$\mathrm{C}$ & 0.35739600 & 3.81232300 & -0.75022700 \\
$\mathrm{H}$ & -0.43142700 & 2.04165400 & 0.15014300 \\
$\mathrm{C}$ & 1.45964200 & 4.42336400 & -1.35524100 \\
$\mathrm{H}$ & 3.50525300 & 4.17513000 & -2.00424100 \\
$\mathrm{H}$ & -0.57777100 & 4.34632400 & -0.61449200 \\
$\mathrm{H}$ & 1.39361800 & 5.45411200 & -1.69214700 \\
& & & \\
\hline
\end{tabular}

F/I-i-IM2

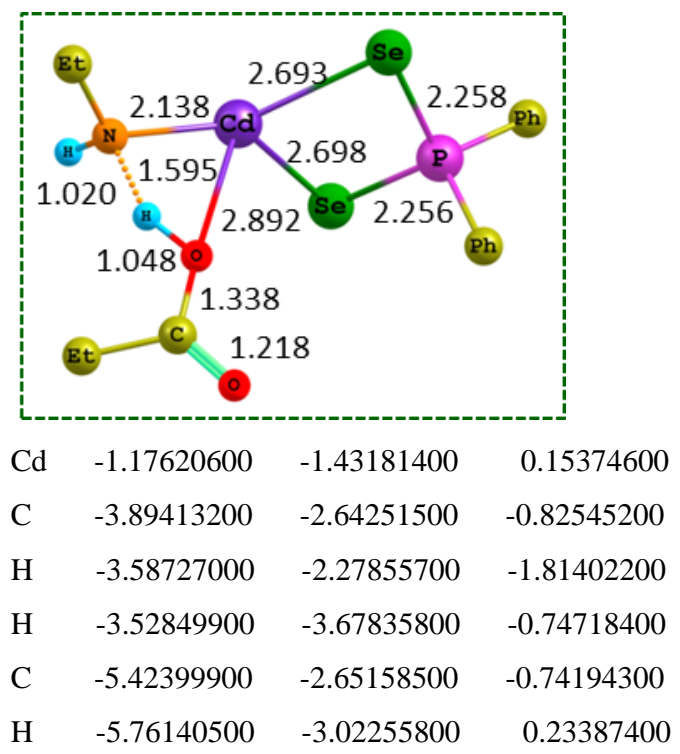




\begin{tabular}{|c|c|c|c|}
\hline & -5.830 & 00 & 00 \\
\hline & -5.85276400 & -3.30295100 & -1.51269300 \\
\hline & -3.26291600 & 2.96569900 & -0.08244200 \\
\hline & -3.73117300 & 1.84906600 & 0.05131200 \\
\hline & -5.14573700 & 1.58651500 & 0.55915700 \\
\hline & 73800 & 500 & 2600 \\
\hline & -5.07837100 & 0.88638700 & 0240200 \\
\hline & -5.89882700 & 2.85474700 & 0.95966100 \\
\hline & -6.90690000 & 2.60581800 & 5700 \\
\hline & -5.37679100 & 3.38436600 & 19800 \\
\hline & -5.98284600 & 3.54334100 & 50400 \\
\hline & -3.28876400 & -1.75779600 & 0.18949000 \\
\hline & -3.58254200 & -2.07154600 & 2900 \\
\hline & -3.01 & 6500 & 7500 \\
\hline & -3.41053900 & 0.18456700 & -0. \\
\hline & 1.71577600 & 0.01956900 & -0.00670500 \\
\hline & 0.63 & 400 & 600 \\
\hline & 0.78808600 & -1.18992400 & -1.67 \\
\hline & 3.49942600 & 72400 & 0.16544800 \\
\hline & 4.19 & -1. & -0 \\
\hline & 4.17136700 & 0.03275500 & 1.33670700 \\
\hline & 5.56557200 & -1.27 & -0.70208600 \\
\hline & 5100 & -1.3 & 7100 \\
\hline & 5.53419900 & -0.22968400 & 1.47890300 \\
\hline & 3.63 & 0 & 00 \\
\hline & 6.23322600 & 29500 & 2200 \\
\hline & 6.10196300 & -1.79558500 & -1.49292100 \\
\hline & 6.04704200 & 0.07144500 & 2.38760000 \\
\hline & 7.29323000 & -1.09256500 & 0.57482000 \\
\hline & 7843400 & 800 & -0.3 \\
\hline & 2.71282900 & 2.58438500 & -0.65379300 \\
\hline & 0.30443300 & 2.40036900 & -0.38954300 \\
\hline & 900 & 0 & 500 \\
\hline & 3.70240100 & 2.14042800 & -0.64681600 \\
\hline & 0.16843800 & 3.75613700 & -0.68302300 \\
\hline & -0.59055400 & 1.82319000 & -0.18187600 \\
\hline & 1.30071700 & 4.52997900 & -0.96108200 \\
\hline & 3.45044600 & 4.54010000 & -1.16364800 \\
\hline & -0.82727200 & 4.188 & -0.688 \\
\hline & 1.19410200 & 5.58706100 & -1.18799300 \\
\hline
\end{tabular}

I-i/H-IM1

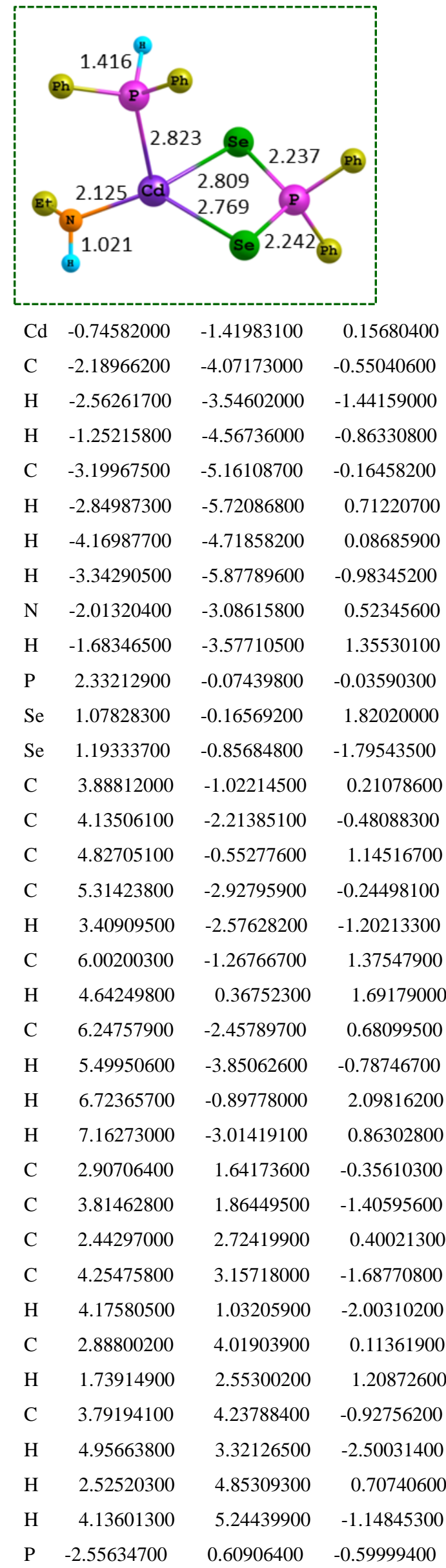




$\begin{array}{lrrr}\mathrm{H} & -2.51111600 & 0.83836700 & -1.99634800 \\ \mathrm{C} & -4.34552900 & 0.25287300 & -0.33954100 \\ \mathrm{C} & -4.70297800 & -1.00329600 & 0.17677600 \\ \mathrm{C} & -5.34681600 & 1.18908800 & -0.65289500 \\ \mathrm{C} & -6.05494900 & -1.31395500 & 0.37017100 \\ \mathrm{H} & -3.93352000 & -1.73773200 & 0.42555200 \\ \mathrm{C} & -6.69074400 & 0.87077900 & -0.45645800 \\ \mathrm{H} & -5.07687800 & 2.16704900 & -1.04199300 \\ \mathrm{C} & -7.04649100 & -0.38317000 & 0.05595100 \\ \mathrm{H} & -6.32619000 & -2.28659100 & 0.77115000 \\ \mathrm{H} & -7.45949900 & 1.59846600 & -0.70146400 \\ \mathrm{H} & -8.09376400 & -0.62871200 & 0.20967500 \\ \mathrm{C} & -2.31789200 & 2.34066800 & -0.03457200 \\ \mathrm{C} & -1.91333500 & 3.35044000 & -0.92178900 \\ \mathrm{C} & -2.48219000 & 2.65380800 & 1.32553900 \\ \mathrm{C} & -1.68956700 & 4.65111100 & -0.45997100 \\ \mathrm{H} & -1.77133900 & 3.12579900 & -1.97533000 \\ \mathrm{C} & -2.26886500 & 3.95492300 & 1.78241800 \\ \mathrm{H} & -2.78149500 & 1.88236400 & 2.03023800 \\ \mathrm{C} & -1.87214900 & 4.95742100 & 0.89063100 \\ \mathrm{H} & -1.37895300 & 5.42290500 & -1.15858400 \\ \mathrm{H} & -2.40686100 & 4.18450300 & 2.83514700 \\ \mathrm{H} & -1.70526900 & 5.96960500 & 1.24790600\end{array}$

\begin{tabular}{|c|c|c|c|}
\hline & 3.16126800 & -6.12905400 & -1. \\
\hline & .55245400 & 1.08588900 & -0.96992500 \\
\hline & -4.03433500 & 0.50850200 & -2.15376600 \\
\hline & -3.86768400 & .42881600 & 0.68931600 \\
\hline & -4.82 & 4800 & 9800 \\
\hline & -3.79988900 & -0.52734300 & -2.37867100 \\
\hline & -4.65703900 & 3.16958800 & -1.56974400 \\
\hline & 1200 & 100 & 5600 \\
\hline & -5.1 & 600 & -2 . \\
\hline & 600 & 00 & 400 \\
\hline & -4.896 & 8600 & 9100 \\
\hline & 8000 & 500 & -3 \\
\hline C & -3.10658200 & .49846300 & 1.82874200 \\
\hline & -2.23267700 & 0.77254000 & 2.89194500 \\
\hline C & -4.48805200 & 400 & $500-2>$ \\
\hline C & -2.73264900 & 500 & 3100 \\
\hline $\mathrm{H}$ & -1.16364700 & 0.82914000 & 2.70699500 \\
\hline C & -4.98371 & 0 & 0 \\
\hline H & -5.17452700 & 0.20751800 & 99000 \\
\hline C & -4.1 & 0 & 00 \\
\hline $\mathrm{H}$ & -2.04736500 & 00 & 100 \\
\hline $\mathrm{H}$ & -6.05325700 & 1500 & 0200 \\
\hline $\mathrm{H}$ & $-4.4 \mathrm{C}$ & 1.05232000 & 00 \\
\hline C & 3.71938500 & 0.04003200 & 0.96175700 \\
\hline $\mathrm{C}$ & 4.32 & 7900 & 00 \\
\hline $\mathrm{C}$ & 9600 & 0 & 00 \\
\hline C & 5.02503200 & 0.31009800 & 2.98846800 \\
\hline $\mathrm{H}$ & 4.26012100 & 1.95123400 & 1.82467600 \\
\hline C & 4.51147000 & -1.90699700 & 2.16706800 \\
\hline $\mathrm{H}$ & 3.31631500 & -2.01001500 & .36933300 \\
\hline $\mathrm{C}$ & 5.12194500 & -1.07841100 & 0300 \\
\hline $\mathrm{H}$ & 5.49543100 & 0.95957300 & 3.72141500 \\
\hline $\mathrm{H}$ & 4.58182300 & -2.98702700 & 2026 \\
\hline H & 5.66870400 & -1.51234300 & 3.94695400 \\
\hline C & 3.39768100 & 2.38094800 & -0.79082200 \\
\hline C & 2.61105500 & 3.52238000 & -0.57913500 \\
\hline $\mathrm{C}$ & 4.69587900 & 2.52402400 & -1.31152200 \\
\hline $\mathrm{C}$ & 3.11949400 & 4.79170600 & -0.87320100 \\
\hline $\mathrm{H}$ & 1.59934600 & 3.41535500 & 010964100 \\
\hline C & 5.19727300 & 3.79160000 & -1.61076000 \\
\hline $\mathrm{H}$ & 5.31589900 & 1.64752200 & -1.4794730 \\
\hline $\mathrm{C}$ & 4.41010700 & 4.92748700 & -1.38914300 \\
\hline $\mathrm{H}$ & 2.50286400 & 5.67002700 & -0.70567100 \\
\hline $\mathrm{H}$ & 6.20001500 & 3.89282600 & -2.0160980 \\
\hline $\mathrm{H}$ & 4.80203200 & 1330600 & 35000 \\
\hline & 1.78447400 & -3.01217700 & -1.29475000 \\
\hline
\end{tabular}


$\begin{array}{llll}\mathrm{H} & 2.16363100 & -2.60458000 & -2.15091800\end{array}$

I-i/H-IM2

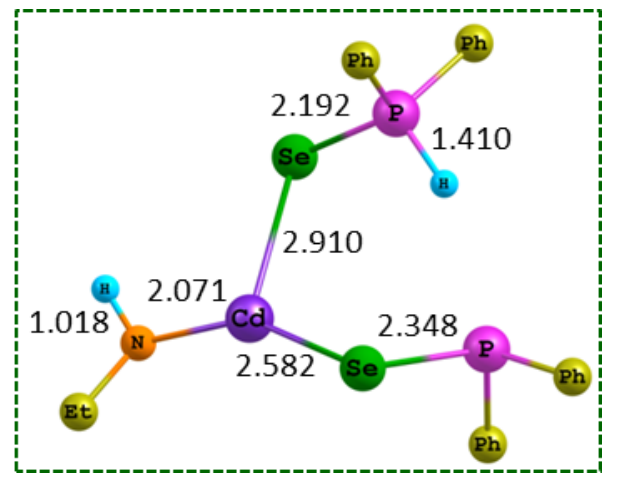

Se $\quad-1.52903000 \quad-0.05599800 \quad-1.65984300$

$\begin{array}{llll}\mathrm{P} & -1.39595000 & 1.44986200 & 0.13631300\end{array}$

$\begin{array}{llll}\text { C } & -2.97242300 & 1.16994600 & 1.06482000\end{array}$

$\begin{array}{llll}\text { C } & -4.24962800 & 1.38631600 & 0.51957200\end{array}$

$\begin{array}{llll}\text { C } & -2.86763400 & 0.69441100 & 2.38148100\end{array}$

$\begin{array}{llll}\text { C } & -5.39307400 & 1.13767200 & 1.27837500\end{array}$

$\begin{array}{llll}\mathrm{H} & -4.34570100 & 1.74263400 & -0.50178500\end{array}$

$\begin{array}{llll}\text { C } & -4.01491400 & 0.44156200 & 3.14212700\end{array}$

$\begin{array}{llll}\mathrm{H} & -1.88504700 & 0.51894200 & 2.81268500\end{array}$

$\begin{array}{llll}\text { C } & -5.27844100 & 0.66428500 & 2.59186400\end{array}$

$\begin{array}{llll}\mathrm{H} & -6.37515400 & 1.30813300 & 0.84553200\end{array}$

$\begin{array}{llll}\mathrm{H} & -3.91819100 & 0.07133200 & 4.15895900\end{array}$

$\begin{array}{llll}\mathrm{H} & -6.17120600 & 0.46845300 & 3.17928300\end{array}$

$\begin{array}{llll}\text { C } & -1.75149900 & 3.06649000 & -0.70200100\end{array}$

$\begin{array}{llll}\text { C } & -2.16820300 & 4.14522200 & 0.10245600\end{array}$

$\begin{array}{llll}\text { C } & -1.49078400 & 3.30963100 & -2.05938900\end{array}$

$\begin{array}{llll}\text { C } & -2.32976400 & 5.42144200 & -0.43862100\end{array}$

$\mathrm{H} \quad-2.37769000 \quad 3.98659500 \quad 1.15708400$

$\begin{array}{llll}\text { C } & -1.65099300 & 4.59001100 & -2.60032300\end{array}$

$\begin{array}{llll}\mathrm{H} & -1.17538200 & 2.49432400 & -2.70298500\end{array}$

$\begin{array}{llll}\text { C } & -2.07077300 & 5.65017000 & -1.79444500\end{array}$

$\mathrm{H} \quad-2.66112700 \quad 6.23650200 \quad 0.19920100$

$\begin{array}{llll}\mathrm{H} & -1.45286500 & 4.75285800 & -3.65643100\end{array}$

$\begin{array}{llll}\mathrm{H} & -2.19798500 & 6.64315900 & -2.21632900\end{array}$

Cd $-0.86268700 \quad-2.22101700 \quad-0.42163200$

Se $\quad 1.52818700 \quad-1.88332800 \quad 1.20160600$

$\begin{array}{llll}\text { C } & -2.31293800 & -4.97784000 & -0.59622100\end{array}$

$\begin{array}{llll}\mathrm{H} & -1.99378200 & -5.33688600 & -1.59234900\end{array}$

H $\quad-3.19053700 \quad-4.34151300 \quad-0.77036800$

$\begin{array}{llll}\text { C } & -2.74236300 & -6.19133700 & 0.23974100\end{array}$

$\mathrm{H} \quad-3.12588400 \quad-5.86945600 \quad 1.21374000$

$\begin{array}{llll}\mathrm{H} & -3.52079400 & -6.77007300 & -0.27446400\end{array}$

$\begin{array}{llll}\mathrm{H} & -1.89136900 & -6.86088600 & 0.41840000\end{array}$

$\begin{array}{llll}\mathrm{P} & 2.28310100 & 0.00743300 & 0.38963300\end{array}$

$\begin{array}{llll}\text { C } & 3.25411000 & -0.10883200 & -1.14653300\end{array}$

$\begin{array}{lrrr}\mathrm{C} & 4.62066100 & -0.42739600 & -1.10882900 \\ \mathrm{C} & 2.60689100 & 0.06653000 & -2.37988900 \\ \mathrm{C} & 5.33661400 & -0.55776400 & -2.29917300 \\ \mathrm{H} & 5.12510500 & -0.56711800 & -0.15698500 \\ \mathrm{C} & 3.33154000 & -0.06552000 & -3.56719000 \\ \mathrm{H} & 1.54374000 & 0.29253100 & -2.41559200 \\ \mathrm{C} & 4.69327500 & -0.37678300 & -3.52851100 \\ \mathrm{H} & 6.39454800 & -0.80104800 & -2.26738100 \\ \mathrm{H} & 2.82901400 & 0.07344200 & -4.51985300 \\ \mathrm{H} & 5.25336800 & -0.47927700 & -4.45350800 \\ \mathrm{C} & 3.27835400 & 0.96072000 & 1.58985400 \\ \mathrm{C} & 3.72608000 & 2.24368600 & 1.23090200 \\ \mathrm{C} & 3.57504000 & 0.44705200 & 2.85788300 \\ \mathrm{C} & 4.46833500 & 3.00100000 & 2.13736900 \\ \mathrm{H} & 3.50091400 & 2.65132000 & 0.24894600 \\ \mathrm{C} & 4.32477500 & 1.20842100 & 3.75959000 \\ \mathrm{H} & 3.21533100 & -0.53873100 & 3.13728300 \\ \mathrm{C} & 4.77029900 & 2.48275800 & 3.40172400 \\ \mathrm{H} & 4.80880600 & 3.99353000 & 1.85758300 \\ \mathrm{H} & 4.55450000 & 0.80461900 & 4.74117000 \\ \mathrm{H} & 5.34915700 & 3.07388500 & 4.10550000 \\ \mathrm{H} & 1.20993200 & 0.85496200 & 0.04748400 \\ \mathrm{~N} & -1.29544200 & -4.18111300 & 0.08981200 \\ \mathrm{H} & -0.45848200 & -4.73909100 & 0.24588500\end{array}$

$\mathrm{I}-\mathrm{i}$

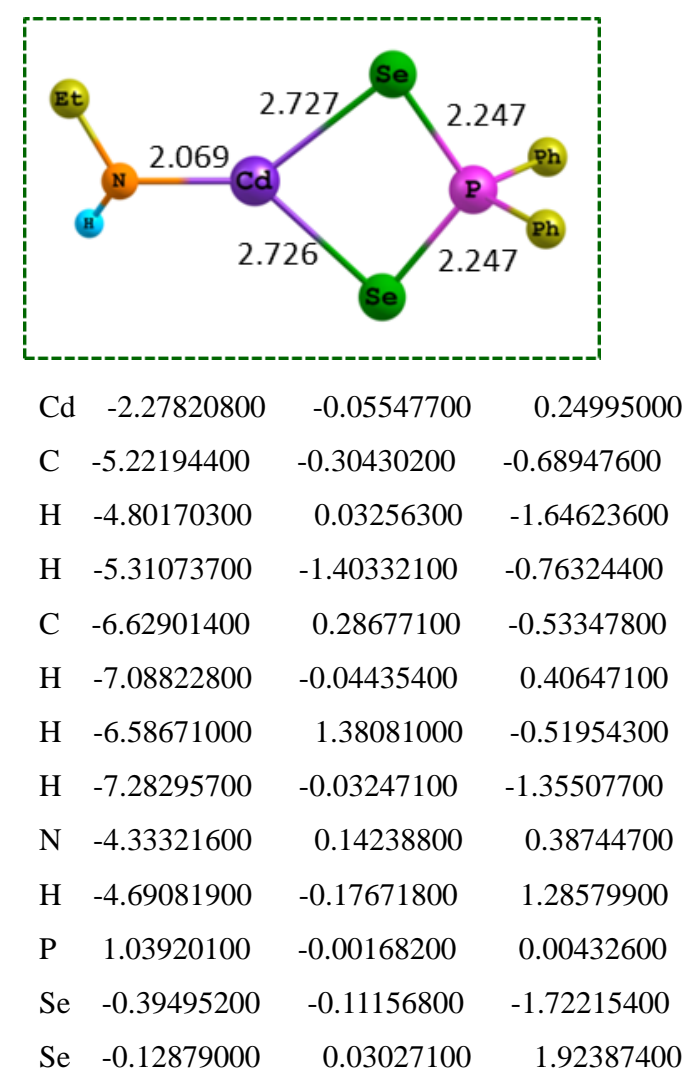




$\begin{array}{lrrr}\mathrm{C} & 2.10443100 & 1.48351700 & -0.14494900 \\ \mathrm{C} & 1.97755900 & 2.56632000 & 0.73398100 \\ \mathrm{C} & 3.03596300 & 1.54385500 & -1.19599700 \\ \mathrm{C} & 2.78152600 & 3.69830900 & 0.56774700 \\ \mathrm{H} & 1.25648000 & 2.52183300 & 1.54416100 \\ \mathrm{C} & 3.83604000 & 2.67461900 & -1.35536600 \\ \mathrm{H} & 3.13800500 & 0.71272400 & -1.88782700 \\ \mathrm{C} & 3.70972600 & 3.75439400 & -0.47377400 \\ \mathrm{H} & 2.67980000 & 4.53302900 & 1.25510700 \\ \mathrm{H} & 4.55474000 & 2.71424600 & -2.16866600 \\ \mathrm{H} & 4.33306100 & 4.63481100 & -0.60093600 \\ \mathrm{C} & 2.20483400 & -1.41676900 & -0.01385400 \\ \mathrm{C} & 3.34719900 & -1.36780000 & 0.80330300 \\ \mathrm{C} & 1.94863000 & -2.55976700 & -0.78194700 \\ \mathrm{C} & 4.22399800 & -2.45175000 & 0.84341100 \\ \mathrm{H} & 3.55526400 & -0.48728400 & 1.40398400 \\ \mathrm{C} & 2.83043900 & -3.64344400 & -0.73636800 \\ \mathrm{H} & 1.06733000 & -2.59797700 & -1.41462400 \\ \mathrm{C} & 3.96681200 & -3.59174400 & 0.07399600 \\ \mathrm{H} & 5.10609400 & -2.40622800 & 1.47539000 \\ \mathrm{H} & 2.62773900 & -4.52478600 & -1.33780900 \\ \mathrm{H} & 4.65112600 & -4.43465500 & 0.10657200\end{array}$

H-ii

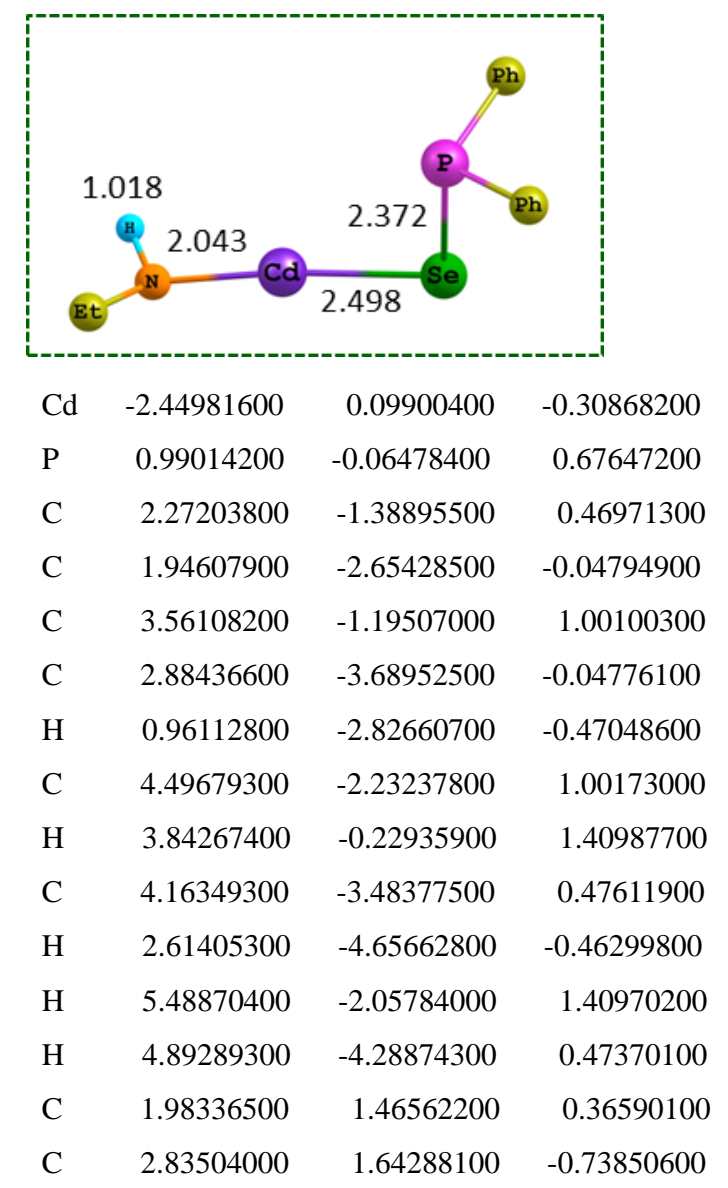

$\begin{array}{lrrr}\mathrm{C} & 1.84984900 & 2.51251400 & 1.29151700 \\ \mathrm{C} & 3.53682800 & 2.83603300 & -0.90776900 \\ \mathrm{H} & 2.94601400 & 0.84668400 & -1.46888100 \\ \mathrm{C} & 2.55548800 & 3.70977200 & 1.12414100 \\ \mathrm{H} & 1.19283900 & 2.39002000 & 2.14923600 \\ \mathrm{C} & 3.39909800 & 3.87282800 & 0.02402000 \\ \mathrm{H} & 4.19018700 & 2.96001500 & -1.76707500 \\ \mathrm{H} & 2.44367500 & 4.50936000 & 1.85116600 \\ \mathrm{H} & 3.94668400 & 4.80144800 & -0.11060700 \\ \mathrm{Se} & -0.21316200 & -0.27600200 & -1.35690300 \\ \mathrm{C} & -5.05927100 & -0.55817800 & 1.19997300 \\ \mathrm{H} & -4.84769800 & -1.57767400 & 0.85255700 \\ \mathrm{H} & -4.70314600 & -0.50701900 & 2.24336800 \\ \mathrm{C} & -6.57585500 & -0.33454900 & 1.18670000 \\ \mathrm{H} & -6.82403400 & 0.67266700 & 1.54387900 \\ \mathrm{H} & -6.97181400 & -0.43901700 & 0.17144500 \\ \mathrm{H} & -7.08284500 & -1.05475700 & 1.84069600 \\ \mathrm{~N} & -4.37765000 & 0.38594700 & 0.30502500 \\ \mathrm{H} & -4.54960500 & 1.34185600 & 0.61061000\end{array}$

H

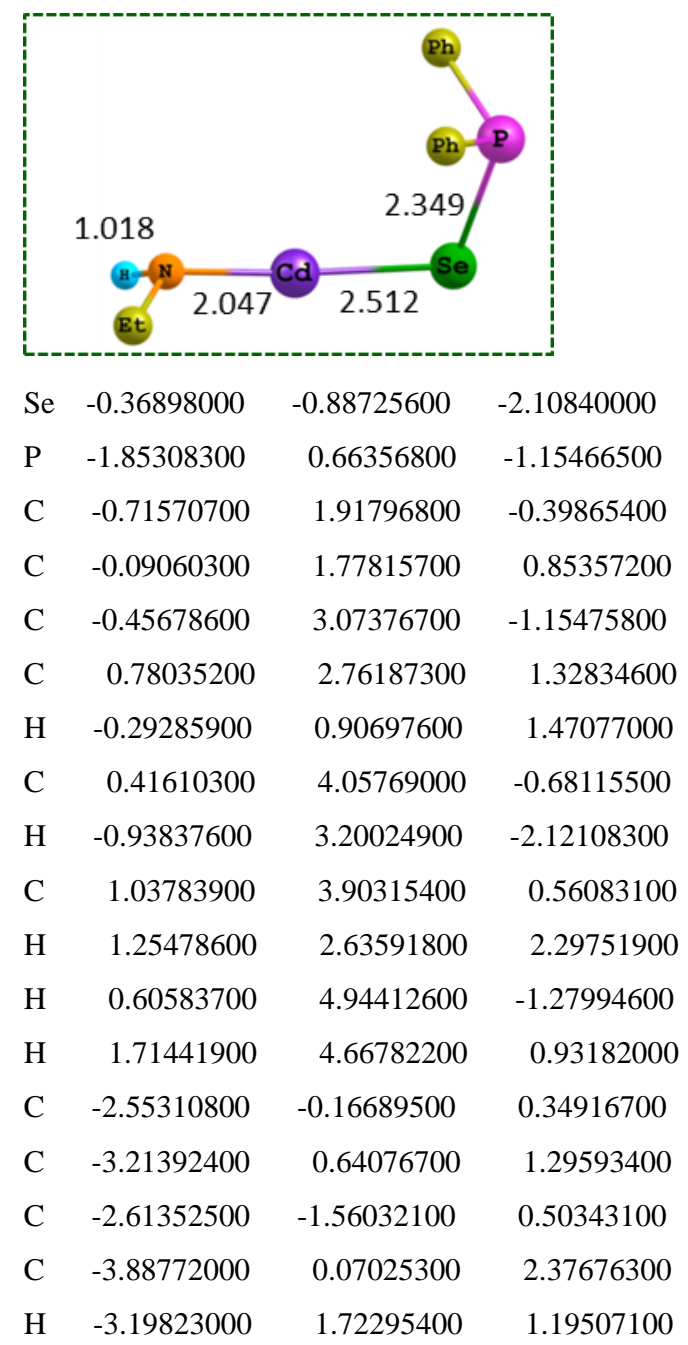




$\begin{array}{lrrr}\mathrm{C} & -3.29454400 & -2.13112700 & 1.58418100 \\ \mathrm{H} & -2.12796600 & -2.20260100 & -0.22487000 \\ \mathrm{C} & -3.92987300 & -1.32013200 & 2.52646200 \\ \mathrm{H} & -4.38110200 & 0.71267800 & 3.10101800 \\ \mathrm{H} & -3.32561800 & -3.21262400 & 1.68581100 \\ \mathrm{H} & -4.45632200 & -1.76409000 & 3.36649000 \\ \mathrm{Cd} & 1.41773900 & -1.09104300 & -0.35384600 \\ \mathrm{~N} & 2.79481200 & -1.20200600 & 1.15699900 \\ \mathrm{H} & 2.96806000 & -2.15858000 & 1.46029500 \\ \mathrm{C} & 4.05828600 & -0.45592900 & 1.10287300 \\ \mathrm{H} & 4.78033800 & -0.86843400 & 0.37688100 \\ \mathrm{H} & 3.83961800 & 0.56756700 & 0.77194400 \\ \mathrm{C} & 4.72799000 & -0.39988000 & 2.48096700 \\ \mathrm{H} & 4.97660900 & -1.40807500 & 2.83515500 \\ \mathrm{H} & 5.65944800 & 0.17834800 & 2.44023600 \\ \mathrm{H} & 4.05906400 & 0.05897800 & 3.21633600\end{array}$

G/H-IM1

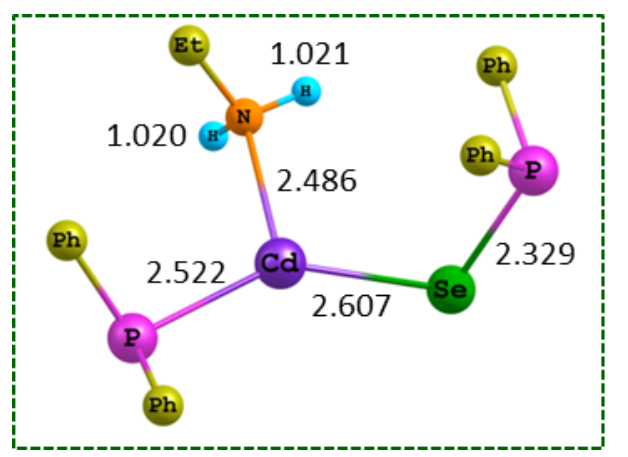

$\begin{array}{lrrr}\mathrm{Se} & 1.52751500 & -1.82019700 & -1.76473600 \\ \mathrm{P} & 2.94809000 & -1.94438800 & 0.07620100 \\ \mathrm{C} & 1.80775400 & -1.84361000 & 1.53630600 \\ \mathrm{C} & 1.18220400 & -0.67152000 & 1.99540700 \\ \mathrm{C} & 1.53381200 & -3.04966900 & 2.20159200 \\ \mathrm{C} & 0.30462200 & -0.70429600 & 3.08062900 \\ \mathrm{H} & 1.39449900 & 0.27738400 & 1.51103800 \\ \mathrm{C} & 0.64941300 & -3.08785500 & 3.28475200 \\ \mathrm{H} & 2.01220800 & -3.96546200 & 1.86406400 \\ \mathrm{C} & 0.03249500 & -1.91574600 & 3.72683100 \\ \mathrm{H} & -0.16634400 & 0.21361200 & 3.42230300 \\ \mathrm{H} & 0.44596700 & -4.03213700 & 3.78187800 \\ \mathrm{H} & -0.65410900 & -1.94271700 & 4.56812500 \\ \mathrm{C} & 3.83264800 & -0.31104500 & 0.14489000 \\ \mathrm{C} & 4.32331000 & 0.18046200 & 1.37034000 \\ \mathrm{C} & 4.22031000 & 0.35021500 & -1.03550500 \\ \mathrm{C} & 5.14884400 & 1.30722400 & 1.41438400 \\ \mathrm{H} & 4.05983000 & -0.31656700 & 2.29948600 \\ \mathrm{C} & 5.05231200 & 1.47385500 & -0.99098300\end{array}$

\begin{tabular}{|c|c|c|c|}
\hline $\mathrm{H}$ & 1800 & 0 & -1.9 \\
\hline & 0 & 0 & 0.23431600 \\
\hline & 50818600 & 67033600 & 2200 \\
\hline & $x_{0}+2$ & 600 & 14500 \\
\hline & 0 & 83174200 & 00 \\
\hline & .71238100 & 60660800 & 1.39155500 \\
\hline $\mathrm{C}$ & & & 400 \\
\hline$C$ & -3.18 & 0 & 0 \\
\hline $\mathrm{C}$ & -4.80 & 00 & 0. \\
\hline$C$ & -3.9 & -3 & 400 \\
\hline & -2.26182900 & 830000 & 1.4754 \\
\hline $\mathrm{C}$ & -5.55723300 & -1.7 & 100 \\
\hline $\mathrm{H}$ & 00 & 047900 & 300 \\
\hline C & -5.13006000 & 800 & 1000 \\
\hline $\mathrm{H}$ & -3.59 & 0 & 5200 \\
\hline $\mathrm{H}$ & -6.4 & 00 & 1. \\
\hline$U$ & -5.7 & 3 & 0 \\
\hline$C$ & -2.87664400 & 4600 & 900 \\
\hline $\mathrm{C}$ & -3 & 0 & 0 \\
\hline $\mathrm{C}$ & -2.76013500 & 1.68997000 & 9500 \\
\hline $\mathrm{C}$ & -3.2 & 00 & 4700 \\
\hline 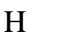 & -3.23781600 & 3.43235400 & -1.57841900 \\
\hline & -2.87288100 & 00 & 400 \\
\hline $\mathrm{H}$ & -2 & 0 & 00 \\
\hline $\mathrm{C}$ & -3.09409900 & 4.04772 & 100 \\
\hline 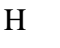 & -3.4 & 900 & 500 \\
\hline$U$ & -2.78547100 & 2.53680200 & 3.27998000 \\
\hline $\mathrm{H}$ & -3.180639 & 0 & 24000 \\
\hline $\mathrm{Cd}$ & -0.27788300 & .02370200 & 4900 \\
\hline $\mathrm{N}$ & 1 & & \\
\hline $\mathrm{H}$ & 2.02753200 & 1.74698400 & -0.95310900 \\
\hline$\Pi$ & 0.95178500 & 2.52047600 & -1.89829400 \\
\hline $\mathrm{C}$ & 0 & 00 & 00 \\
\hline $\mathrm{H}$ & 0.93749800 & 5200 & 500 \\
\hline $\mathrm{H}$ & -0.2 & 0 & 0.0 \\
\hline $\mathrm{C}$ & 100 & 500 & 2400 \\
\hline $\mathrm{H}$ & 1.44365500 & 4.97130800 & $0.85040^{\circ}$ \\
\hline $\mathrm{H}$ & 2.74501500 & 4.02291900 & 0.10802900 \\
\hline$H$ & 1.53838100 & 4.83493700 & 6100 \\
\hline
\end{tabular}

$\begin{array}{llll}\mathrm{H} & 1.53838100 & 4.83493700 & -0.91156100\end{array}$ 
G/H-TS1

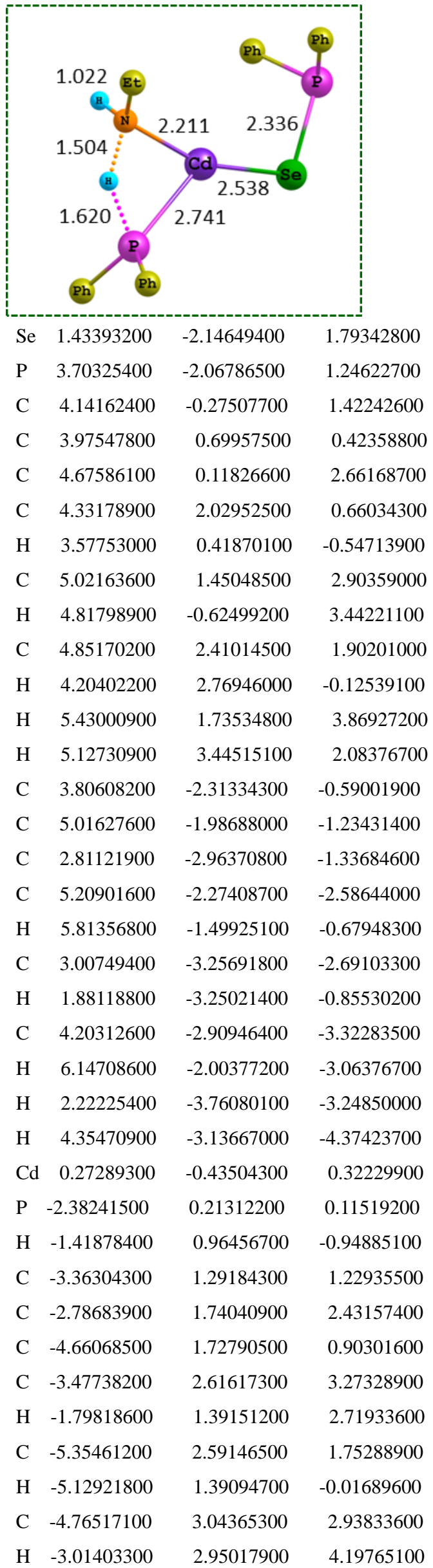

$\begin{array}{rrrc}\mathrm{H} & -6.35706000 & 2.91506700 & 1.48558100 \\ \mathrm{H} & -5.30731400 & 3.71601800 & 3.59702200 \\ \mathrm{C} & -3.58055200 & -0.82871500 & -0.80530400 \\ \mathrm{C} & -3.68437300 & -0.73178800 & -2.20426400 \\ \mathrm{C} & -4.37462100 & -1.77899400 & -0.13604600 \\ \mathrm{C} & -4.55755200 & -1.56168200 & -2.91529600 \\ \mathrm{H} & -3.08686400 & 0.00217700 & -2.73788900 \\ \mathrm{C} & -5.25806800 & -2.59490800 & -0.84438200 \\ \mathrm{H} & -4.29890000 & -1.87810200 & 0.94336400 \\ \mathrm{C} & -5.34935100 & -2.49156500 & -2.23718400 \\ \mathrm{H} & -4.62505000 & -1.47259100 & -3.99618800 \\ \mathrm{H} & -5.86804600 & -3.31876500 & -0.31091300 \\ \mathrm{H} & -6.03227100 & -3.13225200 & -2.78785400 \\ \mathrm{~N} & 0.04290100 & 1.05844100 & -1.29177600 \\ \mathrm{H} & 0.32426500 & 0.70413800 & -2.20779000 \\ \mathrm{C} & 0.55322800 & 2.42851800 & -1.12485700 \\ \mathrm{H} & 0.14112700 & 2.82690600 & -0.18692600 \\ \mathrm{H} & 1.64742500 & 2.44858500 & -1.01032000 \\ \mathrm{C} & 0.14827000 & 3.34779200 & -2.28237700 \\ \mathrm{H} & -0.94130100 & 3.38768300 & -2.38535200 \\ \mathrm{H} & 0.51662300 & 4.36775600 & -2.12015900 \\ \mathrm{H} & 0.56580600 & 2.98863600 & -3.23132400\end{array}$

G/H-IM2

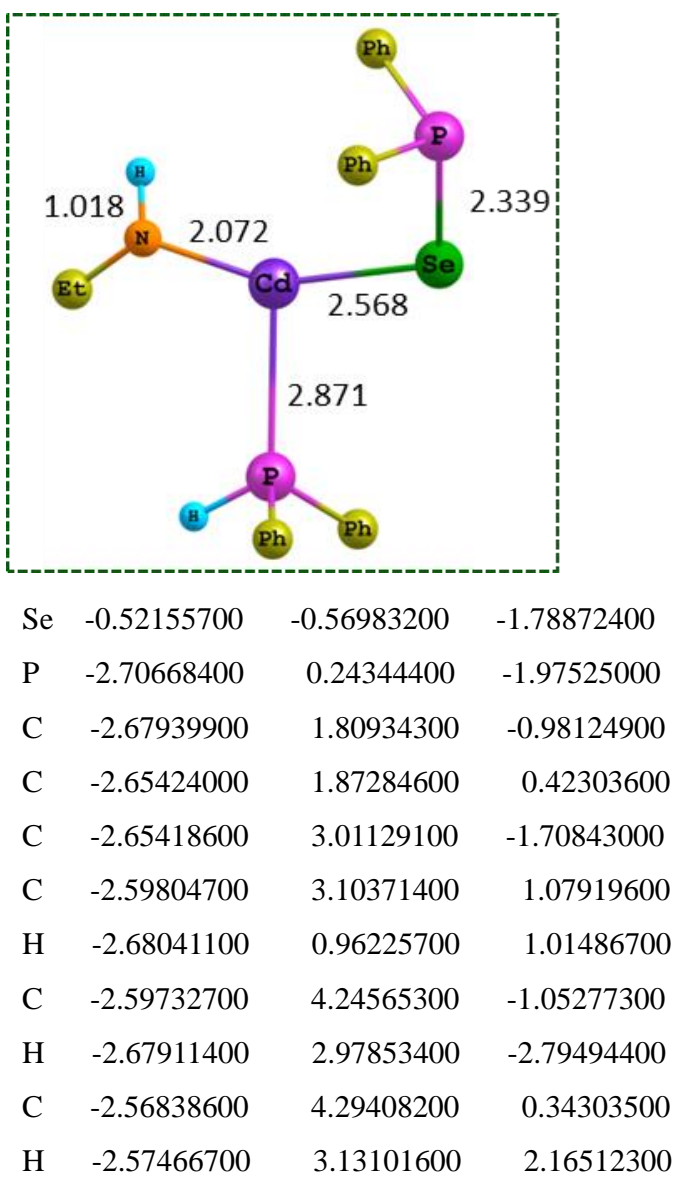




\begin{tabular}{|c|c|c|c|}
\hline & 0 & 00 & 00 \\
\hline & -2.52568600 & 5.25099800 & 0.85593700 \\
\hline & -3.73803400 & 0.86912100 & 0.90691100 \\
\hline & -4.96001600 & .38730100 & .39793900 \\
\hline & -3.43943400 & -2.2 & -0.73863400 \\
\hline & 300 & 00 & 7400 \\
\hline & -5.22 & 6700 & -0.52096700 \\
\hline & -4.3 & & 2800 \\
\hline & -2.50990200 & -2.62895700 & -1.13522000 \\
\hline & -5.52385900 & -2.58 & 36600 \\
\hline & -6.77076100 & -0.8 & 90900 \\
\hline & -4.0 & -4 & 200 \\
\hline & -6.20815400 & -3.2 & 2300 \\
\hline & -0.13 & -0.8 & 00 \\
\hline & 2.68979100 & -0.30918000 & 00 \\
\hline & 3.37770600 & -0.55231200 & 38700 \\
\hline & 3.15572800 & 1.45071400 & 0.53010000 \\
\hline & 2.36651200 & 2.23933000 & 81800 \\
\hline & 4.26888800 & 3300 & 50000 \\
\hline & 2.696 & 100 & 00 \\
\hline & 1.49497200 & 1.81268200 & 37400 \\
\hline & 4.58878300 & 000 & 00 \\
\hline & 4.88774200 & 1.43016600 & 1.83003700 \\
\hline & 3.80434900 & 100 & 1800 \\
\hline & 2.078 & 96000 & 00 \\
\hline & 5.449 & 2000 & 1.44606800 \\
\hline & 4.05 & $0 / 00$ & 57400 \\
\hline & 3.75581700 & -1.29546600 & -0.32984900 \\
\hline & 4.89381900 & -1.97211800 & 0.13898100 \\
\hline & 3. & -1.40628800 & 0 \\
\hline & 5.66980800 & -2.73911700 & -0.73448500 \\
\hline & 5.17868200 & -1.90292200 & 1.18533800 \\
\hline & 4.1 & 2 & 00 \\
\hline & 2.51598300 & -0.90673200 & -2.06488400 \\
\hline & 5.32061300 & -2.83341400 & -2.08428100 \\
\hline & 6.54695800 & -3.25893400 & -0.35952300 \\
\hline & 3.90551600 & -2.23949200 & -3.60360200 \\
\hline & 5.92515900 & -3.42828700 & -2.76280300 \\
\hline & -0.77959900 & -0.94865200 & 2.70209300 \\
\hline & -1.64986900 & -1.46979300 & 2.78723900 \\
\hline & 0.09456100 & -1.25142300 & 3.83346800 \\
\hline & 0.36733400 & -2.32103200 & 3.90570000 \\
\hline & 1.03893300 & -0.70661200 & 3.69890700 \\
\hline & -0.52892000 & -0.82266700 & 5.16915100 \\
\hline & -1.46954200 & -1.35832500 & 5.34768900 \\
\hline & 0.14448900 & 58900 & 34800 \\
\hline
\end{tabular}

$\begin{array}{llll}\mathrm{H} & -0.75031400 & 0.24985100 & 5.16284500\end{array}$ I-i/I-TS1

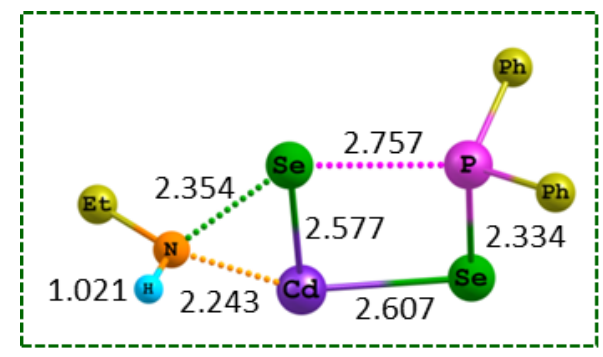

\begin{tabular}{|c|c|c|c|}
\hline & 2.17546600 & -0.21782300 & 0.470018 \\
\hline $\mathrm{C}$ & 3.17011000 & 1.16612300 & -2.42042100 \\
\hline $\mathrm{H}$ & 3.08148000 & 2.07135000 & -1.80789900 \\
\hline $\mathrm{H}$ & 4.24255300 & 2200 & -2.47456800 \\
\hline $\mathrm{C}$ & 2.63489100 & 1.44208500 & -3.82826600 \\
\hline $\mathrm{H}$ & 2.74750800 & 0.56222800 & -4.47327500 \\
\hline 11 & 1.57235300 & 1.70285700 & -3.78778700 \\
\hline $\mathrm{H}$ & 3.18000400 & 2.27182000 & -4.29309500 \\
\hline $\mathrm{N}$ & 2.44385700 & 0.09209700 & -1.73550800 \\
\hline $\mathrm{H}$ & 7100 & -0.75805900 & -2.30091200 \\
\hline $\mathrm{P}$ & -1.02387600 & 0.36350800 & 1.39558800 \\
\hline $\mathrm{Se}$ & 0.82070000 & -0.23 & 2.69737400 \\
\hline $\mathrm{S}$ & 0.21623900 & 0.45140400 & -1.06477000 \\
\hline$C$ & -1.59639900 & 1.96652000 & 75200 \\
\hline$C$ & -1.49482900 & 3.11211900 & 1.29581400 \\
\hline $\mathrm{C}$ & -2.10797300 & 6200 & 3.4 \\
\hline C & -1.90372800 & 4.35704800 & 1.78560400 \\
\hline$\Pi$ & -1.09473400 & 3.02687900 & 89600 \\
\hline$C$ & -2.51508900 & 3.32783200 & 3.88926900 \\
\hline $\mathrm{H}$ & -2.18910800 & 1.20700700 & 4.03785000 \\
\hline$c$ & -2.4 & 600 & 4200 \\
\hline $\mathrm{H}$ & -1.82427100 & 5.23669800 & 1.15306700 \\
\hline $\mathrm{H}$ & -2.90840200 & 3.40917400 & 4.89880600 \\
\hline $\mathrm{H}$ & -2.73145900 & 5.43315500 & 3.46181800 \\
\hline $\mathrm{C}$ & -2.35980900 & -0.82087600 & 1.85941100 \\
\hline $\mathrm{C}$ & -3.69962500 & -0.43126300 & 1.67439800 \\
\hline $\mathrm{C}$ & -2.08580200 & -2.14309400 & 22434 \\
\hline $\mathrm{C}$ & -4.73756200 & -1.34069600 & 1.88524200 \\
\hline $\mathrm{H}$ & -3.93621500 & 0.58391800 & 1.36954600 \\
\hline$C$ & -3.12788000 & -3.05003800 & 2.45237600 \\
\hline $\mathrm{H}$ & -1.05812200 & -2.45772000 & 2.39398500 \\
\hline 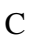 & -4.45610200 & -2.65337600 & 2.27597700 \\
\hline $\mathrm{H}$ & -5.76635300 & -1.02094800 & 1.74453900 \\
\hline $\mathrm{H}$ & -2.89838300 & -4.06692300 & 2.75866600 \\
\hline $\mathrm{H}$ & -5.26476300 & -3.35957200 & 2.44115800 \\
\hline
\end{tabular}




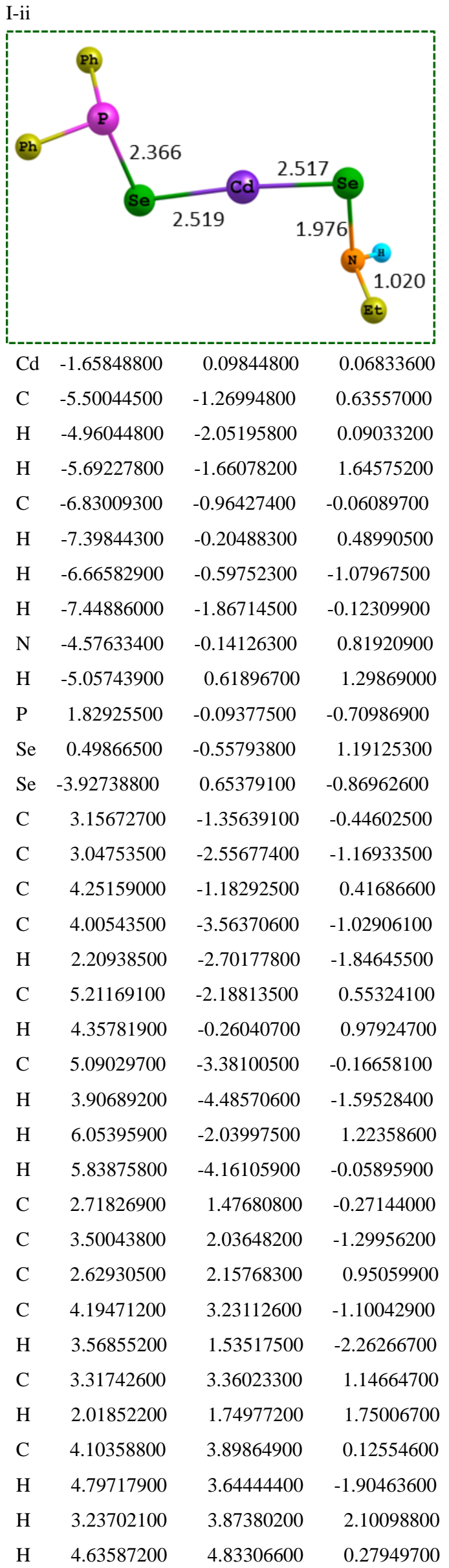

I

\begin{tabular}{|c|c|c|c|}
\hline & $\begin{array}{l}\mathrm{Ph} \\
2.346\end{array}$ & $\underbrace{1.971}_{\mathrm{NE}} 1.020$ & \\
\hline & & & \\
\hline $\mathrm{P}$ & 2.68519800 & 0.28740000 & 1.15654200 \\
\hline $\mathrm{C}$ & 1.94104300 & 1.70357100 & 0.21412700 \\
\hline $\mathrm{C}$ & 1.39541800 & 1.59967800 & -1.07921500 \\
\hline $\mathrm{C}$ & 1.91177300 & 2.95244000 & 0.85801900 \\
\hline $\mathrm{C}$ & 0.82336600 & 2.71306300 & -1.70238200 \\
\hline $\mathrm{H}$ & 1.43745000 & 0.65349900 & -1.61207600 \\
\hline $\mathrm{C}$ & 1.33552500 & 4.06403500 & 0.23680500 \\
\hline $\mathrm{H}$ & 2.33809200 & 3.05179300 & 1.85301200 \\
\hline $\mathrm{C}$ & 0.78811000 & 3.94683600 & -1.04402300 \\
\hline $\mathrm{H}$ & 0.40972100 & 2.61609600 & -2.70245200 \\
\hline $\mathrm{H}$ & 1.31914800 & 5.02084200 & 0.75107500 \\
\hline $\mathrm{H}$ & 0.34270500 & 4.81057800 & -1.52908300 \\
\hline $\mathrm{C}$ & 3.16748200 & -0.85771000 & -0.22193800 \\
\hline $\mathrm{C}$ & 4.16528600 & -0.40054000 & -1.10557000 \\
\hline $\mathrm{C}$ & 2.71911300 & -2.18035100 & -0.34629300 \\
\hline $\mathrm{C}$ & 4.67461300 & -1.23413400 & -2.10177200 \\
\hline $\mathrm{H}$ & 4.54506800 & 0.61494900 & -1.01976100 \\
\hline $\mathrm{C}$ & 3.23597200 & -3.01802700 & -1.34144400 \\
\hline $\mathrm{H}$ & 1.96647500 & -2.55907900 & 0.33825600 \\
\hline $\mathrm{C}$ & 4.21028100 & -2.54867500 & -2.22408100 \\
\hline $\mathrm{H}$ & 5.43688400 & -0.85882900 & -2.77904800 \\
\hline $\mathrm{H}$ & 85100 & -4.03902700 & -1.42 \\
\hline $\mathrm{H}$ & 4.60993100 & -3.19987000 & -2.99620500 \\
\hline $\mathrm{Cd}$ & -0.98224300 & -0.18286800 & 0.44568600 \\
\hline $\mathrm{Se}$ & -2.93324100 & 0.18611000 & -1.11024900 \\
\hline $\mathrm{N}$ & -3.79962600 & -1.35880100 & -0.24641400 \\
\hline $\mathrm{H}$ & -3.92305400 & 1200 & -0.99373600 \\
\hline $\mathrm{C}$ & -5.10310200 & -1.05127200 & 0.35948200 \\
\hline $\mathrm{H}$ & -4.93535200 & -0.29119000 & 1.13070400 \\
\hline $\mathrm{H}$ & -5.40967900 & -1.97052000 & 0.88023800 \\
\hline $\mathrm{C}$ & -6.21876500 & -0.60086500 & -0.58928000 \\
\hline $\mathrm{H}$ & -6.42123600 & -1.36432900 & -1.35043000 \\
\hline $\mathrm{H}$ & -7.14827300 & -0.42468400 & -0.03510600 \\
\hline $\mathrm{H}$ & -5.94930100 & 0.32942900 & -1.10040300 \\
\hline
\end{tabular}




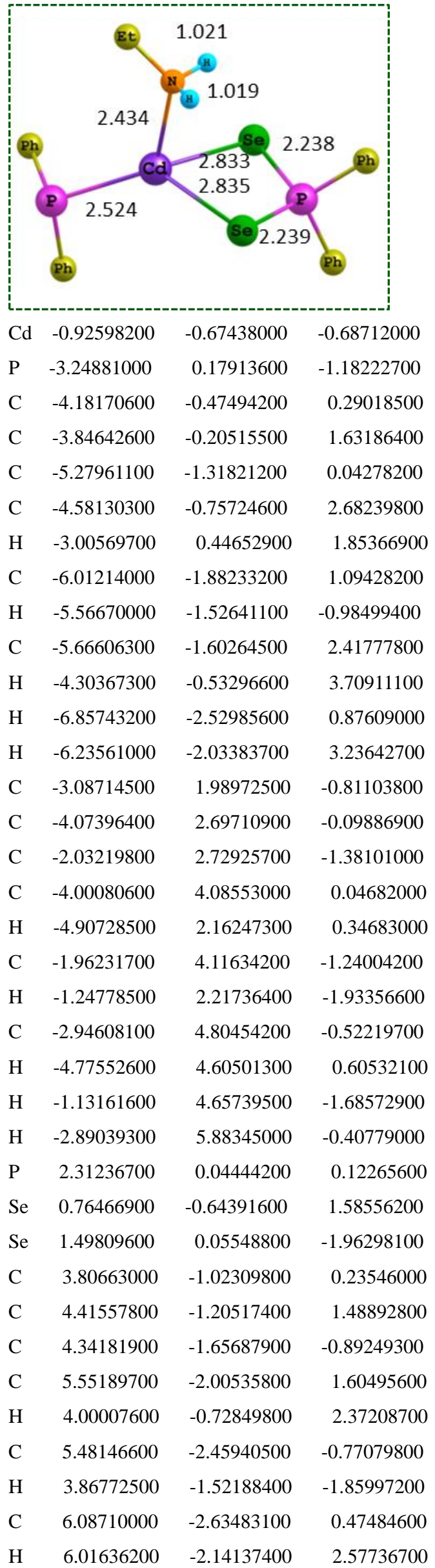

$\begin{array}{lrrr}\mathrm{H} & 5.89208400 & -2.94490000 & -1.65158900 \\ \mathrm{H} & 6.97093200 & -3.25948400 & 0.56830300 \\ \mathrm{C} & 2.91893000 & 1.72150000 & 0.56581500 \\ \mathrm{C} & 4.14496900 & 2.17055800 & 0.04734100 \\ \mathrm{C} & 2.14549800 & 2.57921600 & 1.35878900 \\ \mathrm{C} & 4.59024100 & 3.46329800 & 0.32577200 \\ \mathrm{H} & 4.75241300 & 1.51580300 & -0.57033400 \\ \mathrm{C} & 2.59492300 & 3.87405000 & 1.63152500 \\ \mathrm{H} & 1.20009500 & 2.23303200 & 1.76430700 \\ \mathrm{C} & 3.81555700 & 4.31784400 & 1.11741500 \\ \mathrm{H} & 5.54068000 & 3.80252600 & -0.07606200 \\ \mathrm{H} & 1.98888600 & 4.53231900 & 2.24720600 \\ \mathrm{H} & 4.16362000 & 5.32424800 & 1.33207600 \\ \mathrm{C} & -1.42798200 & -4.08798900 & -1.20702400 \\ \mathrm{H} & -2.05047800 & -3.80654600 & -2.06276700 \\ \mathrm{H} & -0.95156900 & -5.04810900 & -1.45115200 \\ \mathrm{C} & -2.28706200 & -4.23969600 & 0.04469800 \\ \mathrm{H} & -1.67694200 & -4.52222800 & 0.91078100 \\ \mathrm{H} & -2.81382800 & -3.31105800 & 0.28480500 \\ \mathrm{H} & -3.03625000 & -5.02357100 & -0.10443900 \\ \mathrm{~N} & -0.40640700 & -3.02714300 & -1.03022300 \\ \mathrm{H} & 0.22545600 & -2.98948000 & -1.82908200 \\ \mathrm{H} & 0.17827900 & -3.22292500 & -0.21688200\end{array}$

E-i/I-TS1

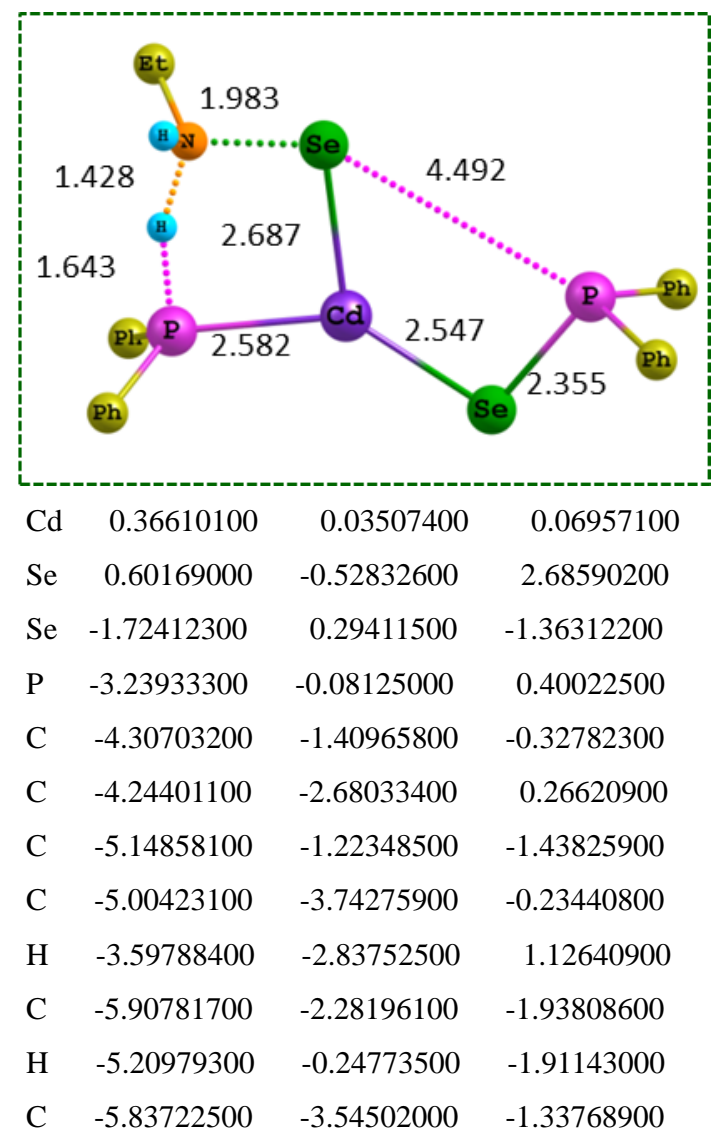




\begin{tabular}{|c|c|c|c|}
\hline & 00 & -4 & 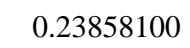 \\
\hline & -6.55365000 & -2.12365800 & 2.79767800 \\
\hline & -6.42978100 & -4.36757300 & -1.72874500 \\
\hline & -4.38075000 & 1.38111300 & 0.29552000 \\
\hline & -4.00042400 & 2.62587000 & -0.22757700 \\
\hline & 211400 & 2200 & 2500 \\
\hline & -4.85932500 & 3.72750500 & -0.14728500 \\
\hline & -3.03473600 & 2.72988900 & -0.71228400 \\
\hline & -6.49939000 & 2.37041400 & $0 \mathrm{c}$ \\
\hline & -5.96205300 & 600 & 1.33103200 \\
\hline & -6.11089700 & 3.60547300 & 1000 \\
\hline & -4.5 & 00 & 00 \\
\hline & -7.47115200 & 800 & 6700 \\
\hline & 78800 & 200 & 00 \\
\hline & 3.01858300 & 11500 & 3000 \\
\hline & 2.58193400 & -0.45709800 & 24300 \\
\hline & 2.80 & 0 & 00 \\
\hline & 3.20653900 & 4500 & 3.28323600 \\
\hline & 8900 & 300 & 2 \\
\hline & 2.74 & 400 & 4 \\
\hline & 4.71552900 & -1.50837300 & 3.45673300 \\
\hline & 5.16308000 & 400 & 38 \\
\hline & 5.19986900 & -1.26145000 & 2.50664500 \\
\hline & 4.94104400 & 300 & 3700 \\
\hline I & 2.93368600 & 13700000 & 19600 \\
\hline & 3.793 & -1.13811900 & 8600 \\
\hline & 4.75387900 & 300 & 9000 \\
\hline & 3.46656000 & -2.49128000 & -0.96661900 \\
\hline & 5.37488700 & -1.83531300 & -2.89072400 \\
\hline & 0 & 212 & -2.34 \\
\hline & 4.08751300 & -3.49851700 & -1.70558800 \\
\hline & 2.71827600 & -2.76186700 & -0.22462700 \\
\hline & 700 & -3 . & 00 \\
\hline & 6.11494200 & -1.57131000 & -3.64133700 \\
\hline & 3.81927400 & -4.53636100 & -1.52839400 \\
\hline & 5.52910400 & -3.95929800 & -3.24785900 \\
\hline & 3.70090100 & 1.76082700 & -0.55196400 \\
\hline & 4.92873100 & 2.12053600 & 0.03452300 \\
\hline & 3.06129500 & 2.68361800 & -1.39750500 \\
\hline & 5.50377600 & 3.36666900 & 52400 \\
\hline & 5.43746000 & 1.42145700 & 0.69252800 \\
\hline & 3.63485600 & 3.93280000 & -1.65273300 \\
\hline & 2.11261700 & 2.42368600 & -1.85837800 \\
\hline & 4.856 & 4.27587900 & -1.06766300 \\
\hline & 6.45285500 & 3.62899000 & 0.23452500 \\
\hline & 3.12629900 & 3600 & 727200 \\
\hline
\end{tabular}

\section{E-i/I-IM2}

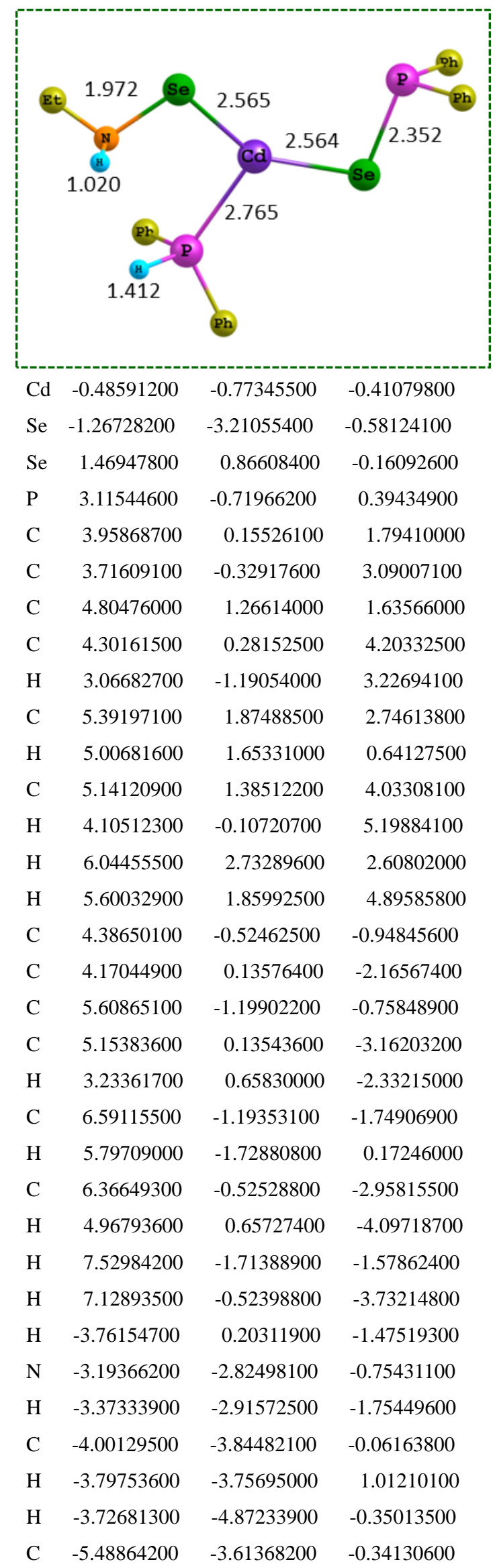




$\begin{array}{lrrc}\mathrm{H} & -6.09585100 & -4.35146300 & 0.19492000 \\ \mathrm{H} & -5.79922100 & -2.61306600 & -0.02388600 \\ \mathrm{H} & -5.71137800 & -3.71655400 & -1.41046600 \\ \mathrm{P} & -2.79234800 & 0.74005400 & -0.60051100 \\ \mathrm{C} & -3.73320100 & 0.84565400 & 0.98171200 \\ \mathrm{C} & -4.15413800 & 2.06402800 & 1.53306500 \\ \mathrm{C} & -3.98398400 & -0.35215600 & 1.67275000 \\ \mathrm{C} & -4.82714300 & 2.08262100 & 2.75874100 \\ \mathrm{H} & -3.96028400 & 2.99678500 & 1.01323600 \\ \mathrm{C} & -4.66430400 & -0.32668100 & 2.89175100 \\ \mathrm{H} & -3.65633300 & -1.29536400 & 1.24403100 \\ \mathrm{C} & -5.08539500 & 0.88987400 & 3.43845600 \\ \mathrm{H} & -5.14843900 & 3.03131600 & 3.17931100 \\ \mathrm{H} & -4.85826500 & -1.25711700 & 3.41785700 \\ \mathrm{H} & -5.60715000 & 0.90761000 & 4.39101500 \\ \mathrm{C} & -2.67972400 & 2.44854200 & -1.25181600 \\ \mathrm{C} & -3.66056200 & 2.96785300 & -2.11443800 \\ \mathrm{C} & -1.57979400 & 3.25071700 & -0.90369600 \\ \mathrm{C} & -3.54515400 & 4.26704300 & -2.61464900 \\ \mathrm{H} & -4.51652100 & 2.35992500 & -2.39458300 \\ \mathrm{C} & -1.47242700 & 4.55287700 & -1.39795200 \\ \mathrm{H} & -0.80069400 & 2.86157500 & -0.25361400 \\ \mathrm{C} & -2.45259800 & 5.06189700 & -2.25471200 \\ \mathrm{H} & -4.30754000 & 4.65651000 & -3.28324500 \\ \mathrm{H} & -0.61703800 & 5.16202400 & -1.12137500 \\ \mathrm{H} & -2.36317300 & 6.07167900 & -2.64480900\end{array}$

H/I-IM1

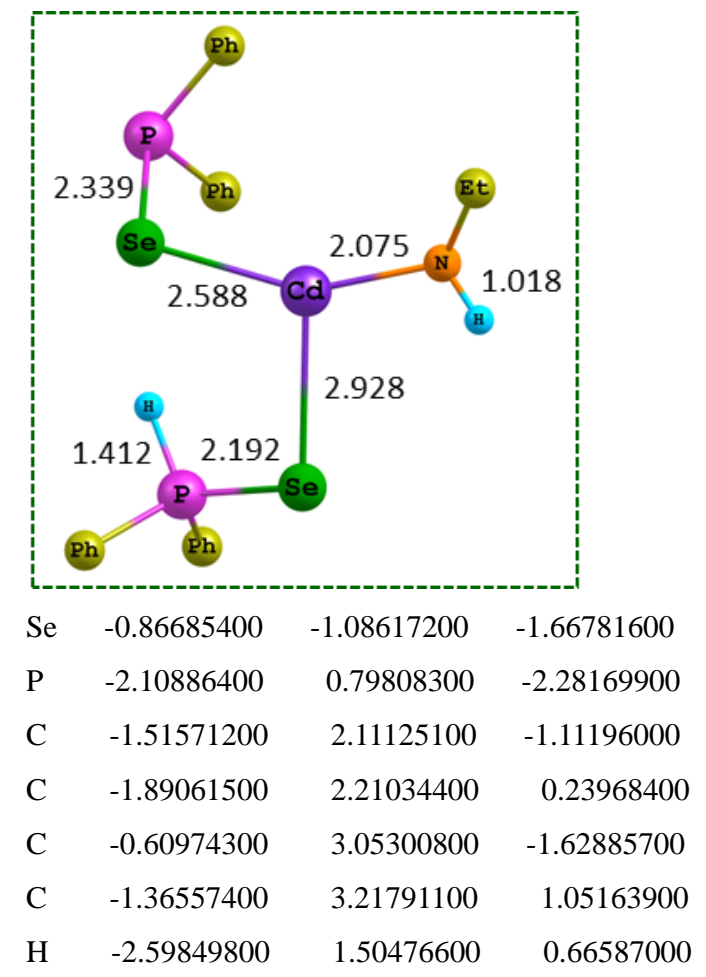

\begin{tabular}{|c|c|c|c|}
\hline C & -0.08182600 & 4.06169300 & -0.81645800 \\
\hline $\mathrm{H}$ & -0.31970300 & 2.99628100 & -2.67540500 \\
\hline C & -0.45857800 & 4.14542300 & 0.52638800 \\
\hline $\mathrm{H}$ & -1.66406700 & 3.27428100 & 2.09480100 \\
\hline $\mathrm{H}$ & 0.61635600 & 4.78213200 & -1.23399700 \\
\hline $\mathrm{H}$ & -0.05176400 & 4.92889400 & 1.15971700 \\
\hline $\mathrm{C}$ & -3.82671900 & 0.48805700 & -1.65329800 \\
\hline$c$ & -4.69692100 & 1.58803300 & -1.52174800 \\
\hline $\mathrm{C}$ & -4.35060800 & -0.80134000 & -1.47224000 \\
\hline- & -6.04141900 & 1.40186800 & -1.19627600 \\
\hline $\mathrm{H}$ & -4.32293900 & 2.59840000 & -1.66516500 \\
\hline$c$ & -5.69950100 & -0.98691900 & -1.15163700 \\
\hline 11 & -3.69961200 & -1.66360900 & -1.57999000 \\
\hline 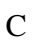 & -6.54928600 & 0.11189900 & -1.00900200 \\
\hline $\mathrm{H}$ & -6.69191000 & 2.26569500 & -1.08841000 \\
\hline $\mathrm{H}$ & -6.08206900 & -1.99427400 & -1.01041100 \\
\hline $\mathrm{H}$ & -7.59569300 & -0.03297300 & -0.75576000 \\
\hline $\mathrm{Cd}$ & -1.09931100 & -1.23110800 & 0.90545500 \\
\hline $\mathrm{Se}$ & 1.66840500 & -1.20141300 & 1.85969700 \\
\hline $\mathrm{C}$ & -3.53072400 & -1.78729600 & 2.78437500 \\
\hline $\mathrm{H}$ & -3.49262100 & -2.88976600 & 2.86045100 \\
\hline $\mathrm{H}$ & -4.10744100 & -1.56403700 & 1.87768600 \\
\hline C & -4.29520500 & -1.24703800 & 4.00088100 \\
\hline $\mathrm{H}$ & -4.41167000 & -0.16031900 & 3.92975900 \\
\hline $\mathrm{H}$ & -5.29016900 & -1.70454200 & 4.07717300 \\
\hline $\mathrm{H}$ & -3.75408100 & -1.46708600 & 4.93000000 \\
\hline $\mathrm{P}$ & 2.57537700 & -0.20995100 & 0.12789800 \\
\hline 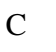 & 3.71907000 & -1.23380500 & -0.84733600 \\
\hline $\mathrm{C}$ & 4.99663700 & -1.55040600 & -0.35895100 \\
\hline $\mathrm{C}$ & 3.28607100 & -1.75870300 & -2.07500500 \\
\hline $\mathrm{C}$ & 5.84051800 & -2.37368300 & -1.10414500 \\
\hline 11 & 5.33112300 & -1.15556100 & 0.59629600 \\
\hline $\mathrm{C}$ & 4.13688700 & -2.58486200 & -2.81467300 \\
\hline $\mathrm{H}$ & 2.29012100 & -1.53333300 & -2.44736900 \\
\hline$c$ & 5.41161200 & -2.89123300 & -2.33194100 \\
\hline $\mathrm{H}$ & 6.82984500 & -2.61435000 & -0.72636900 \\
\hline $\mathrm{H}$ & 3.79994700 & -2.98855200 & -3.76482000 \\
\hline $\mathrm{H}$ & 6.07009500 & -3.53450700 & -2.90844800 \\
\hline $\mathrm{C}$ & 3.44284000 & 1.35058800 & 0.52967300 \\
\hline $\mathrm{C}$ & 3.28548000 & 1.94997700 & 1.78559000 \\
\hline $\mathrm{C}$ & 4.23854000 & 1.97190300 & -0.44821800 \\
\hline $\mathrm{C}$ & 3.92271200 & 3.16244200 & 2.06354700 \\
\hline $\mathrm{H}$ & 2.67029200 & 1.46625600 & 2.53827600 \\
\hline $\mathrm{C}$ & 4.86773700 & 3.18508100 & -0.16587500 \\
\hline $\mathrm{H}$ & 4.37298700 & 1.51144800 & -1.42280300 \\
\hline $\mathrm{C}$ & 4.71190600 & 3.78034200 & 1.09071200 \\
\hline
\end{tabular}




$\begin{array}{rrrr}\mathrm{H} & 3.80092900 & 3.62064900 & 3.04059400 \\ \mathrm{H} & 5.48180200 & 3.66187500 & -0.92424600 \\ \mathrm{H} & 5.20681700 & 4.72225000 & 1.30942900 \\ \mathrm{H} & 1.57195800 & 0.10054700 & -0.81610600 \\ \mathrm{~N} & -2.21161400 & -1.16525800 & 2.65612600 \\ \mathrm{H} & -1.64771400 & -1.37183800 & 3.47836800\end{array}$

H/I-TS1

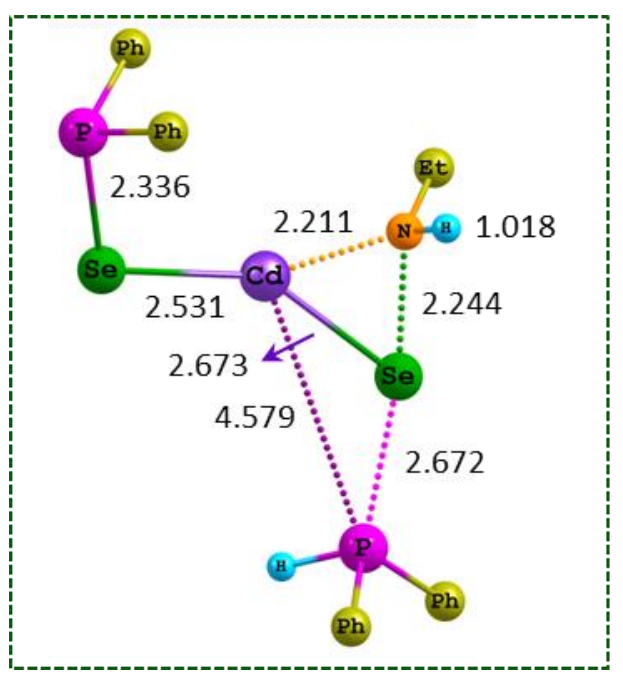

$\begin{array}{lrrr}\mathrm{Se} & 2.11296800 & 1.10392700 & -1.92445600 \\ \mathrm{P} & 4.32527400 & 1.06859000 & -1.17401400 \\ \mathrm{C} & 4.68532100 & -0.73088200 & -0.91623300 \\ \mathrm{C} & 4.38469300 & -1.45452600 & 0.25050700 \\ \mathrm{C} & 5.30446000 & -1.40387300 & -1.98383500 \\ \mathrm{C} & 4.69265600 & -2.81400500 & 0.34337500 \\ \mathrm{H} & 3.92029100 & -0.95391100 & 1.09489900 \\ \mathrm{C} & 5.60396800 & -2.76583800 & -1.89545900 \\ \mathrm{H} & 5.55008300 & -0.85617300 & -2.89022200 \\ \mathrm{C} & 5.29965800 & -3.47451100 & -0.72998300 \\ \mathrm{H} & 4.45953100 & -3.35778000 & 1.25496900 \\ \mathrm{H} & 6.08034500 & -3.26930300 & -2.73212700 \\ \mathrm{H} & 5.53762900 & -4.53190500 & -0.65536600 \\ \mathrm{C} & 4.29002600 & 1.70773400 & 0.56763000 \\ \mathrm{C} & 5.43140600 & 1.50277300 & 1.36856700 \\ \mathrm{C} & 3.26557700 & 2.52619900 & 1.06837700 \\ \mathrm{C} & 5.52694000 & 2.07226700 & 2.63911800 \\ \mathrm{H} & 6.25002300 & 0.88881800 & 1.00204900 \\ \mathrm{C} & 3.36427700 & 3.10074900 & 2.34049900 \\ \mathrm{H} & 2.38850600 & 2.72129100 & 0.45886500 \\ \mathrm{C} & 4.49108900 & 2.87313900 & 3.13272400 \\ \mathrm{H} & 6.41246600 & 1.89169800 & 3.24256900 \\ \mathrm{H} & 2.55634200 & 3.72794800 & 2.70786500 \\ \mathrm{Hd} & 4.56679600 & 3.31843300 & 4.12067300 \\ & 0.79080900 & -0.25979300 & -0.25106700\end{array}$

\begin{tabular}{|c|c|c|c|}
\hline e & -1.62 & -100 & 0.61646800 \\
\hline $\mathrm{C}$ & 0.629 & -3.05662700 & 9900 \\
\hline $\mathrm{H}$ & 1.69469500 & -3.23252700 & 170927000 \\
\hline & 0.441 & -3.4 & 9600 \\
\hline $\mathrm{C}$ & -0 & -3.7 & 0 \\
\hline $\mathrm{H}$ & 0 & $365 \div$ & PO \\
\hline $\mathrm{H}$ & -0.01523500 & -4.87130200 & 2.50529000 \\
\hline $\mathrm{H}$ & -0.03466000 & 2600 & (2) \\
\hline$P$ & -3.765 & -0.00 & -0.6 \\
\hline $\mathrm{C}$ & -4.17912600 & 1.75602600 & 200 \\
\hline $\mathrm{C}$ & -4.22075400 & 2.2 & 1500 \\
\hline $\mathrm{C}$ & -4.40003200 & 266614300 & -1.37168300 \\
\hline C & -4.50 & 100 & 7800 \\
\hline $\mathrm{H}$ & -4.02 & 400 & 1.0 \\
\hline $\mathrm{C}$ & -4.66948800 & 2200 & -1.0 \\
\hline $\mathrm{H}$ & -4.30 & 300 & -2 \\
\hline $\mathrm{C}$ & -4.72676200 & 4.45743900 & 0.225548 \\
\hline H & -4.53813200 & 0500 & 1800 \\
\hline $\mathrm{H}$ & -4.83 & 0 & 0 \\
\hline $\mathrm{H}$ & -4.93 & 0 & 0 \\
\hline $\mathrm{C}$ & -5.33158100 & -0.93663600 & -0.34240900 \\
\hline $\mathrm{C}$ & -5.2 & -2 & 0 \\
\hline $\mathrm{C}$ & -6.60304900 & -0.37212800 & -0.53672800 \\
\hline$C$ & -6.38459800 & -3.03297200 & 0.29359200 \\
\hline $\mathrm{H}$ & -4.2 & -2 & 025622500 \\
\hline$C$ & -7.75287300 & -1.13459100 & -0.32005300 \\
\hline$\Pi$ & -6.69715100 & 0.663 & -0.85050600 \\
\hline $\mathrm{C}$ & -7.64576000 & -2.46716400 & 0.09235400 \\
\hline $\mathrm{H}$ & -6.29479700 & -4.06485100 & 0.62125900 \\
\hline $\mathrm{H}$ & -8.73155800 & -0.68806200 & -0.47267300 \\
\hline $\mathrm{H}$ & -8.54170500 & -3.05 & 9300 \\
\hline $\mathrm{H}$ & -3.73234300 & 0.02810000 & -2.05363800 \\
\hline in & 0.36702300 & -1.61419200 & 1.44395000 \\
\hline IT & 0.43356000 & -1.21022700 & 2.37641000 \\
\hline
\end{tabular}

$\begin{array}{llll}\text { Se } & -1.62655700 & -1.00138800 & 0.61646800\end{array}$

$\begin{array}{llll}\mathrm{H} & 1.69469500 & -3.23252700 & 1.70927900\end{array}$

$\begin{array}{llll}\mathrm{H} & 0.44120900 & -3.46335500 & 0.49179600\end{array}$

$\begin{array}{llll}\text { C } & -0.23160200 & -3.79651400 & 2.51987800\end{array}$

$\mathrm{H} \quad-1.29561800 \quad-3.65409700 \quad 2.30609600$

H $\quad-0.03466000 \quad-3.42902600 \quad 3.53470000$

$\begin{array}{llll}\mathrm{P} & -3.76514200 & -0.00292200 & -0.63689200\end{array}$

$\begin{array}{llll}\text { C } & -4.17912600 & 1.75602600 & -0.32507200\end{array}$

$\begin{array}{llll}\text { C } & -4.22075400 & 2.21891700 & 1.00151500\end{array}$

$\begin{array}{llll}\text { C } & -4.40003200 & 2.66614300 & -1.37168300\end{array}$

$\mathrm{H} \quad-4.02218400 \quad 1.53152400 \quad 1.81949900$

$\begin{array}{llll}\text { C } & -4.66948800 & 4.01052200 & -1.09689600\end{array}$

$\begin{array}{llll}\text { H } & -4.36125500 & 2.32848300 & -2.40393900\end{array}$

$\begin{array}{llll}\text { C } & -4.72676200 & 4.45743900 & 0.22554800\end{array}$

$\begin{array}{llll}\mathrm{H} & -4.53813200 & 3.90250500 & 2.30371800\end{array}$

$\mathrm{H} \quad-4.83548000 \quad 4.70457000 \quad-1.91599600$

$\begin{array}{llll}\mathrm{H} & -4.93785700 & 5.50137100 & 0.43898500\end{array}$

C $\quad-5.23160500 \quad-2.27037300 \quad 0.08281700$

$\begin{array}{llll}\text { C } & -6.60304900 & -0.37212800 & -0.53672800\end{array}$

$-6.38459800-3.03297200$

$\begin{array}{llll}\mathrm{H} & -4.25145600 & -2.70586200 & 0.25622500\end{array}$

$\begin{array}{llll}\mathrm{H} & -6.69715100 & 0.66330900 & -0.85050600\end{array}$

$\begin{array}{llll}\text { C } & -7.64576000 & -2.46716400 & 0.09235400\end{array}$

$\begin{array}{llll}\mathrm{H} & -8.73155800 & -0.68806200 & -0.47267300\end{array}$

$\begin{array}{llll}\mathrm{H} & -8.54170500 & -3.05804700 & 0.26089300\end{array}$

$\begin{array}{llll}\mathrm{N} & 0.36702300 & -1.61419200 & 1.44395000\end{array}$

$\begin{array}{llll}\mathrm{H} & 0.43356000 & -1.21022700 & 2.37641000\end{array}$ 


\section{H/I-IM2}

\begin{tabular}{|c|c|c|c|}
\hline & $\begin{array}{r}2.338 \\
2.559\end{array}$ & $2.567 \int_{\mathrm{Se}}^{\mathrm{N}} 1.962$ & \\
\hline 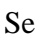 & -0.98558400 & -0.17092700 & -2.58084300 \\
\hline & -3.28433400 & -0.18956300 & -2.15459400 \\
\hline $\mathrm{C}$ & -3.57397800 & 1.38438200 & -1.22146400 \\
\hline C & -3.21136100 & 1.60198900 & 0.11982300 \\
\hline $\mathrm{C}$ & -4.17260400 & 2.43198400 & -1.94118800 \\
\hline C & -3.44501000 & 2.84288900 & 0.71711800 \\
\hline $\mathrm{H}$ & -2.75797000 & 0.81174700 & 0.71741000 \\
\hline $\mathrm{C}$ & -4.40219800 & 3.67440300 & -1.34160400 \\
\hline $\mathrm{H}$ & -4.45892200 & 2.27314700 & -2.97796500 \\
\hline $\mathrm{C}$ & -4.03717100 & 3.88234500 & -0.00956700 \\
\hline $\mathrm{H}$ & -3.16023700 & 2.99801900 & 1.75432700 \\
\hline $\mathrm{H}$ & -4.86582300 & 4.47357400 & -1.91354400 \\
\hline $\mathrm{H}$ & -4.21442800 & 4.84546400 & 0.46146500 \\
\hline $\mathrm{C}$ & -3.54377600 & -1.47244500 & -0.83981400 \\
\hline $\mathrm{C}$ & -4.69212100 & -1.39106700 & -0.02840400 \\
\hline C & -2.73880200 & -2.61871600 & -0.73814100 \\
\hline $\mathrm{C}$ & -5.00816500 & -2.41131700 & 0.87070300 \\
\hline$\Pi$ & -5.34017100 & -0.52134500 & -0.08960700 \\
\hline $\mathrm{C}$ & -3.05627700 & -3.64094000 & 0.16182400 \\
\hline $\mathrm{H}$ & -1.85668800 & -2.71050200 & -1.36471900 \\
\hline $\mathrm{C}$ & -4.18971900 & -3.54119100 & 0.97260000 \\
\hline $\mathrm{H}$ & -5.89491800 & -2.32177700 & 1.49274000 \\
\hline $\mathrm{H}$ & -2.41460000 & -4.51582800 & 0.22641100 \\
\hline $\mathrm{H}$ & -4.43679900 & -4.33581700 & 1.67118400 \\
\hline
\end{tabular}

\begin{tabular}{|c|c|c|c|}
\hline $\mathrm{Cd}$ & 300 & 5800 & -0.2 \\
\hline $\mathrm{Se}$ & 0.28185500 & .17923600 & 8100 \\
\hline C & -2.06799100 & 0.45993700 & 3.81038100 \\
\hline $\mathrm{H}$ & -3.16748200 & 0.41835700 & 3.81479900 \\
\hline $\mathrm{H}$ & -1.78938900 & 1.51168400 & 3.67614900 \\
\hline C & -1.53505000 & -0.05542700 & 5.15302700 \\
\hline $\mathrm{H}$ & -0.44281500 & 0.00877400 & 5.19693700 \\
\hline $\mathrm{H}$ & -1.94322300 & 0.53587000 & 5.98158900 \\
\hline $\mathrm{H}$ & -1.82172300 & -1.10193900 & 1333200 \\
\hline $\mathbf{r}$ & 2.87995300 & 0.17741100 & -0.967 \\
\hline $\mathrm{C}$ & 3.89002600 & -1.33359500 & -0.68256000 \\
\hline$C$ & 6100 & -2.04495900 & 0.51808600 \\
\hline $\mathrm{C}$ & 4.80137700 & -1.80577000 & -1.64173100 \\
\hline $\mathrm{C}$ & 4.48502500 & -3.19374800 & 0.76363600 \\
\hline $\mathrm{H}$ & 3.00835300 & -1.71242800 & 1.26090400 \\
\hline $\mathrm{C}$ & 5.54783500 & -2.961 & 12700 \\
\hline$H$ & 4.92946200 & -1.27610600 & 67900 \\
\hline $\mathrm{C}$ & 5.39466900 & -3.65362500 & -0.19281300 \\
\hline $\mathrm{H}$ & 9800 & -3 & 6300 \\
\hline $\mathrm{H}$ & 6.24690700 & -3.31892300 & -2.14793000 \\
\hline $\mathrm{H}$ & 5.97571900 & -4.55168200 & -0.00420600 \\
\hline $\mathrm{C}$ & 3.85695500 & 1.58178400 & -0.28854300 \\
\hline $\mathrm{C}$ & 3.49789200 & 2.12233200 & 0.95653300 \\
\hline $\mathrm{C}$ & 4.94895800 & 2.12797200 & -0.98459000 \\
\hline$C$ & 4.23050400 & 3.17987600 & 1.50362300 \\
\hline $\mathrm{H}$ & 2.64173100 & 1.72984300 & 1.49943900 \\
\hline $\mathrm{C}$ & 5.67267700 & 3.18992800 & -0.43961500 \\
\hline $\mathrm{H}$ & 5.23633700 & 1.72586600 & -1.95211100 \\
\hline $\mathrm{C}$ & 5.31685500 & 3.71462700 & 0.80752400 \\
\hline$\Pi$ & 3.94388700 & 5100 & 2. \\
\hline $\mathrm{H}$ & 6.51230600 & 3.60749200 & -0.98795500 \\
\hline $\mathrm{H}$ & 5.88090000 & 4.54112400 & 1.23009500 \\
\hline $\mathrm{H}$ & 3.11998000 & 0.35739700 & 54100 \\
\hline $\mathrm{N}$ & -1.65153000 & -0.26270800 & 2.60149200 \\
\hline $\mathrm{H}$ & -1.86062900 & -1.25473000 & 2.71266200 \\
\hline
\end{tabular}


Figure S8. Arrhenius plots of calculated rate constants for the crucial reaction step.

The rate constants $k(T)$ have been evaluated according to conventional transition state theory (TST) $k^{\prime}(T)$, including tunneling correction $\kappa(\mathrm{T})$ based on Winger's formulation ${ }^{1,2}$ :

$$
\begin{gathered}
k^{\prime}(T)=\frac{k_{B} T}{h c^{0}} e^{\frac{-\Delta G^{\neq}}{k_{B} T}} \\
\kappa(T)=1+\frac{1}{24}\left|\frac{h \omega^{\mp}}{k_{B} T}\right|^{2} \\
k(T)=\kappa(T) k^{\prime}(T)
\end{gathered}
$$

where $k_{\mathrm{B}}$ is the Boltzmann constant, $T$ is the absolute temperature, $h$ is the Planck constant, $c^{\circ}$ is the standard concentration $\left(1 \mathrm{~mol} \mathrm{dm}^{-3}\right), \Delta G^{\ddagger}$ is the activation Gibbs free energy barrier, and $\omega^{\neq}$is the imaginary frequency of TS.

1. Eyring, H. The Activated Complex in Chemical Reactions. J. Chem. Phys. 1935, 3, 107-115.

2. Wigner, E. Calculation of the Rate of Elementary Association Reactions. J. Chem. Phys. 1937, 5, 720-725.

For reaction pathway RP-CFE-iG, the TDI and TDTS are E-i $+\mathrm{HPPh}_{2}$ and E-i/G-TS1, respectively.

the rate constant of $\mathbf{E}-\mathbf{i}+\mathrm{HPPh}_{2} \rightarrow \mathbf{E}-\mathbf{i} / \mathbf{G}-\mathbf{T S 1}\left(k_{\mathrm{G}}\right)\left(\right.$ in s $\left.^{-1} \mathrm{~mol}^{-1} \mathrm{dm}^{3}\right)$ :

$$
k_{\mathrm{G}}=5.554 \times 10^{2} \exp (-44,741 / R T)
$$

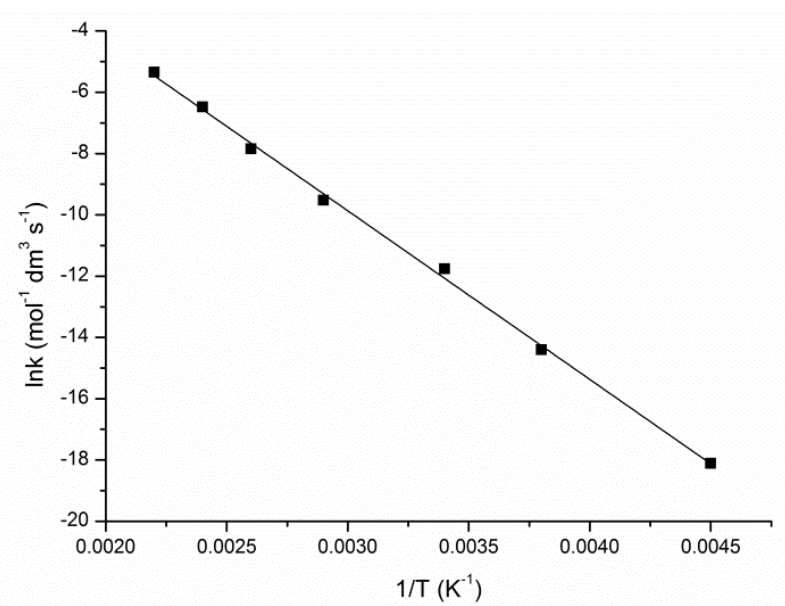

For reaction pathway RP-CEH and RP-CFEH, the TDI and TDTS are $\mathbf{E}+\mathrm{RNH}_{2}$ and $\mathbf{E} / \mathbf{H}-\mathbf{T S 1}$, respectively.

the rate constant of $\mathbf{E}+\mathrm{RNH}_{2} \rightarrow \mathbf{E} / \mathbf{H}-\mathbf{T S 1}\left(k_{\mathrm{H}}\right)\left(\right.$ in s $\left.^{-1} \mathrm{~mol}^{-1} \mathrm{dm}^{3}\right)$ :

$$
k_{\mathrm{H}}=1.459 \times 10^{5} \exp (-93,682 / R T)
$$

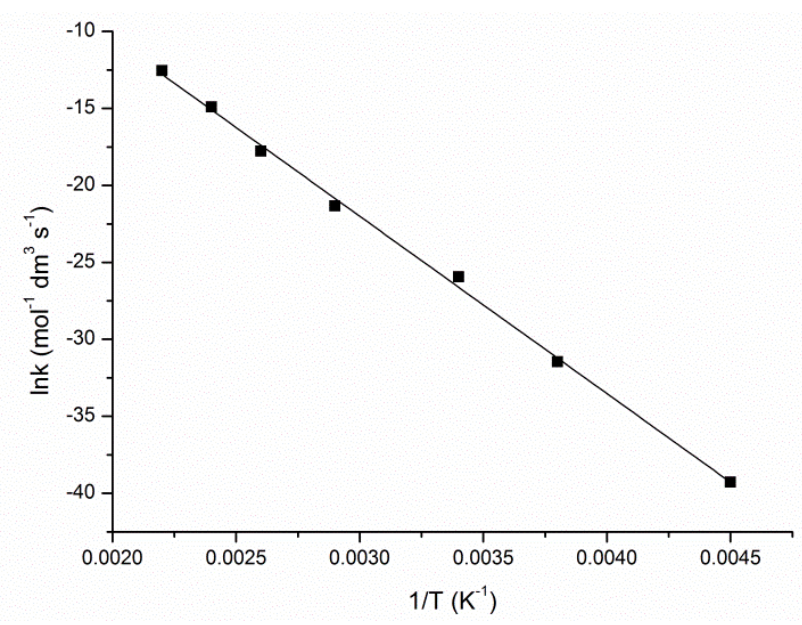


For reaction pathway RP-CFE-iI, the TDI and TDTS are E-i $+\mathrm{RNH}_{2}$ and E-i/I-TS1, respectively. the rate constant of $\mathbf{E}-\mathbf{i}+\mathrm{RNH}_{2} \rightarrow \mathbf{E}-\mathbf{i} / \mathbf{I}-\mathbf{T S 1}\left(k_{\mathrm{I}}\right)\left(\right.$ in s $\left.\mathrm{mol}^{-1} \mathrm{dm}^{3}\right)$ :

$$
k_{\mathrm{I}}=5.334 \times 10^{5} \exp (-125,250 / R T)
$$

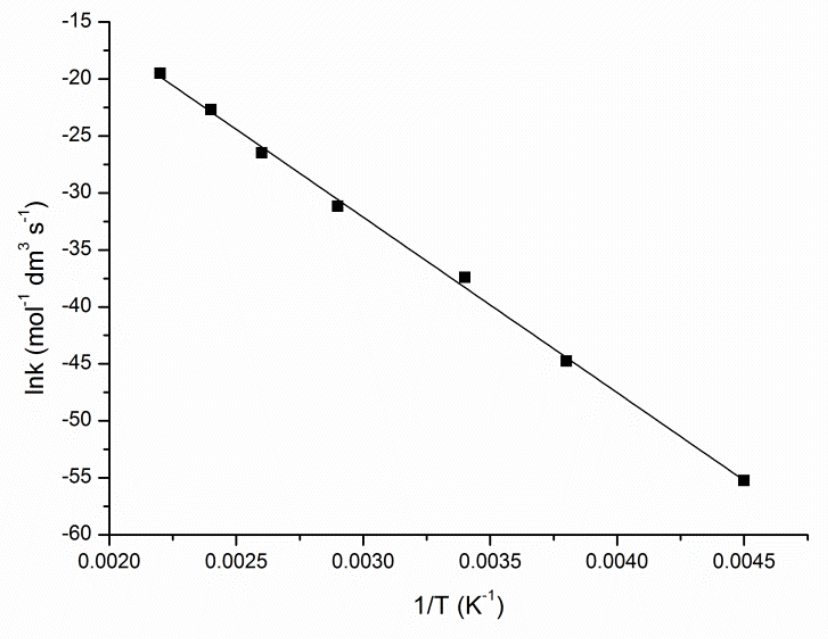


Table S9. Snapshot and standard orientation of all the species in the ODE solution calculated at the M06/6-31++G(d, p), SDD level.

\begin{tabular}{|c|c|c|c|}
\hline \multicolumn{4}{|c|}{$\mathrm{s}-\mathrm{Cd}(\mathrm{OA})_{2}$} \\
\hline $\mathrm{Cd}$ & 0.00049800 & -0.12373700 & -0.01956500 \\
\hline $\mathrm{O}$ & -2.04601100 & 0.69732600 & -0.62321600 \\
\hline $\mathrm{O}$ & -1.90458400 & -0.85243800 & 0.93978600 \\
\hline$C$ & -2.60082600 & -0.06725700 & 0.21946300 \\
\hline $\mathrm{C}$ & -4.09431900 & -0.07478500 & 0.39784900 \\
\hline $\mathrm{H}$ & -4.42058100 & -1.11552900 & 0.25065700 \\
\hline $\mathrm{H}$ & -4.28241800 & 0.13493600 & 1.46199500 \\
\hline $\mathrm{C}$ & -4.85953300 & 0.87850400 & -0.49841100 \\
\hline $\mathrm{H}$ & -5.93638900 & 0.79941100 & -0.31135100 \\
\hline $\mathrm{H}$ & -4.56398600 & 1.91903000 & -0.32333200 \\
\hline $\mathrm{H}$ & -4.68404400 & 0.66131200 & -1.55786500 \\
\hline $\mathrm{O}$ & 2.04180600 & 0.69705500 & 0.60289400 \\
\hline $\mathrm{O}$ & 1.91165500 & -0.83641300 & -0.97702500 \\
\hline $\mathrm{C}$ & 2.60267100 & -0.05820300 & -0.24399900 \\
\hline $\mathrm{C}$ & 4.09728200 & -0.06225800 & -0.41291000 \\
\hline $\mathrm{H}$ & 4.29171800 & 0.17401500 & -1.47044400 \\
\hline $\mathrm{H}$ & 4.42282100 & -1.10625500 & -0.29007900 \\
\hline $\mathrm{C}$ & 4.85724500 & 0.86891600 & 0.51071700 \\
\hline $\mathrm{H}$ & 4.67577300 & 0.62595500 & 1.56362700 \\
\hline $\mathrm{H}$ & 5.93515900 & 0.79425100 & 0.32800000 \\
\hline $\mathrm{H}$ & 4.56263800 & 1.91345400 & 0.35977800 \\
\hline
\end{tabular}

$\begin{array}{lrc}\text { H }-2.40082000 & -3.72402000 & -0.84916900 \\ \text { H }-4.29886800 & -2.93987700 & 0.55138100 \\ \text { Se-0.21787400 } & 2.61544100 & -0.05514300 \\ \text { H } 0.07954600 & 0.45614200 & -2.14158100 \\ & & \\ \text { s-C-IM1 } & & \end{array}$

O $\quad 0.73371500 \quad 1.36770100 \quad-1.53430600$

O $\quad 1.98287200 \quad 2.21884300 \quad 0.08196700$

C $\quad 1.21252100 \quad 2.36342000 \quad-0.91092700$

C $\quad 0.84889400 \quad 3.74833400 \quad-1.39132700$

$\mathrm{H} \quad 1.14544500 \quad 3.79812400 \quad-2.45023000$

$\mathrm{H} \quad-0.25116700 \quad 3.80176500 \quad-1.39961400$

$\begin{array}{llll}\text { C } & 1.44394800 & 4.89432500 & -0.59721300\end{array}$

H $1.11670200 \quad 5.85815700 \quad-1.00634000$

$\begin{array}{llll}\mathrm{H} & 1.13873700 & 4.85288100 & 0.45505300\end{array}$

$\mathrm{H} \quad 2.53969200 \quad 4.87270100 \quad-0.62028100$

$\begin{array}{llll}\text { O } & 3.93528000 & -0.99219000 & 0.79069200\end{array}$

$\begin{array}{llll}\text { O } & 3.35042800 & -1.45680000 & -1.29031100\end{array}$

$\begin{array}{llll}\text { C } & 4.15561900 & -1.56637400 & -0.31466800\end{array}$

$\begin{array}{llll}\text { C } & 5.39656100 & -2.40279600 & -0.51014200\end{array}$

$\begin{array}{llll}\mathrm{H} & 5.93039000 & -1.97074400 & -1.37052800\end{array}$

H $\quad 5.05900000 \quad-3.39332100 \quad-0.85092900$

s- $\mathrm{SePPh}_{2} \mathrm{H}$

$\begin{array}{llll}\mathrm{P} & -0.01865800 & 0.59348300 & -0.73348700\end{array}$

$\begin{array}{lllll}\text { C } & 6.30178500 & -2.51227600 & 0.70088700\end{array}$

$\mathrm{H} \quad 5.78153400 \quad-2.97247500 \quad 1.54935500$

$\begin{array}{llll}\text { C } & 1.48260400 & -0.27495100 & -0.20052600\end{array}$

$\begin{array}{llll}\text { C } & 1.72354300 & -0.46702000 & 1.16323600\end{array}$

$\begin{array}{llll}\text { C } & 2.39733500 & -0.74002400 & -1.14708600\end{array}$

C $\quad 2.86931100 \quad-1.13848600 \quad 1.57343300$

$\mathrm{H} \quad 1.01282200 \quad-0.08741300 \quad 1.90064600$

$\begin{array}{llll}\text { C } & 3.54693300 & -1.40900100 & -0.73087600\end{array}$

$\begin{array}{llll}\mathrm{H} & 2.21369800 & -0.58174400 & -2.21139700\end{array}$

$\begin{array}{llll}\text { C } & 3.78107400 & -1.60839100 & 0.62710800\end{array}$

H $\quad 3.05638500 \quad-1.29219900 \quad 2.63549400$

H $\quad 4.26059300 \quad-1.77261200 \quad-1.46915400$

$\begin{array}{llll}\mathrm{H} & 4.67948400 & -2.13174200 & 0.95245600\end{array}$

C $-1.39971900 \quad-0.51801900 \quad-0.30624700$

$\begin{array}{lll}\mathrm{C}-2.46628000 & -0.07612600 & 0.47382000\end{array}$

C $-1.37647900 \quad-1.83342400 \quad-0.78388800$

$\begin{array}{lll}\text { C }-3.50616700 & -0.95047800 & 0.78477700\end{array}$

$\mathrm{H}-2.47919500 \quad 0.95493300 \quad 0.83206000$

$\begin{array}{lll}\mathrm{C}-2.41863600 & -2.70068000 & -0.47636900\end{array}$

$\mathrm{H}-0.54121900 \quad-2.18394700 \quad-1.39440500$

$\begin{array}{lll}\text { C }-3.48341700 & -2.25896800 & 0.30979200\end{array}$

H $-4.33833400 \quad-0.60426000 \quad 1.39647900$

$\mathrm{H} \quad 7.18223100 \quad-3.12503200 \quad 0.47129500$

$\mathrm{H} \quad 6.65159800 \quad-1.52637300 \quad 1.02889400$

P $-1.59501800 \quad-0.67873500 \quad-0.36504500$

$\begin{array}{llll}\text { C } & -2.84018900 & -1.95460600 & -0.70774500\end{array}$

C $\quad-2.68289500 \quad-3.26607200 \quad-0.25865600$

C $\quad-3.96326400 \quad-1.59790200 \quad-1.46480800$

C $\quad-3.65159300 \quad-4.21990800 \quad-0.56221400$

$\mathrm{H} \quad-1.80685200 \quad-3.53946900 \quad 0.33072100$

C $\quad-4.92219200 \quad-2.55696800 \quad-1.76942100$

$\mathrm{H} \quad-4.09286700 \quad-0.57132000 \quad-1.81262800$

C $\quad-4.76691500 \quad-3.86688100 \quad-1.31665500$

$\mathrm{H} \quad-3.53064900 \quad-5.24226800 \quad-0.20697600$

$\mathrm{H} \quad-5.79520000 \quad-2.28089200 \quad-2.35913900$

$\mathrm{H} \quad-5.52246500 \quad-4.61548700 \quad-1.55210300$

C $\quad-2.49271900 \quad 0.82690500 \quad 0.05986800$

C $\quad-2.43913400 \quad 1.93162800 \quad-0.79297800$

C $\quad-3.26013500 \quad 0.86475100 \quad 1.22844900$

$\begin{array}{llll}\text { C } & -3.16951000 & 3.07578400 & -0.47716300\end{array}$

$\mathrm{H} \quad-1.81654100 \quad 1.89902100 \quad-1.68899100$

C $\quad-3.98467100 \quad 2.00981100 \quad 1.53550400$ 


$\begin{array}{cccc}\mathrm{H} & -3.28887100 & -0.00055300 & 1.89432800 \\ \mathrm{C} & -3.93907400 & 3.11371900 & 0.68243200 \\ \mathrm{H} & -3.13289200 & 3.94067400 & -1.13864200 \\ \mathrm{H} & -4.58433700 & 2.04409200 & 2.44398000 \\ \mathrm{H} & -4.50768900 & 4.01007700 & 0.92742300 \\ \mathrm{Se} & -0.14553300 & -1.34149000 & 1.14554500 \\ \mathrm{H} & -0.96991100 & -0.36169800 & -1.59117900\end{array}$

s-C-TS1

\begin{tabular}{|c|c|c|c|}
\hline $\mathrm{Cd}$ & -1.88938800 & 0.13481700 & -0.66938100 \\
\hline $\mathrm{O}$ & 0.15755300 & 2.50021000 & 1.19430400 \\
\hline $\mathrm{O}$ & -1.95372200 & 1.85425000 & 0.75305000 \\
\hline C & -1.09878300 & 2.57969700 & 1.33509800 \\
\hline $\mathrm{C}$ & -1.64867600 & 3.62909600 & 2.27465300 \\
\hline $\mathrm{H}$ & -2.31974500 & 4.25936500 & 1.67059300 \\
\hline $\mathrm{H}$ & -2.31075100 & 3.10228600 & 2.97844000 \\
\hline $\mathrm{C}$ & -0.61815500 & 4.46618900 & 3.00570000 \\
\hline $\mathrm{H}$ & -1.11122800 & 5.20172800 & 3.65280100 \\
\hline $\mathrm{H}$ & 0.03015300 & 3.84603900 & 3.63618200 \\
\hline $\mathrm{H}$ & 0.02729700 & 5.01075100 & 2.30665300 \\
\hline $\mathrm{O}$ & -4.07242800 & -0.53904900 & -0.76009100 \\
\hline $\mathrm{O}$ & -2.74133600 & -1.56291000 & 0.67888900 \\
\hline $\mathrm{C}$ & -3.88312700 & -1.39851200 & 0.15399000 \\
\hline $\mathrm{C}$ & -5.02265400 & -2.26393200 & 0.62942200 \\
\hline $\mathrm{H}$ & -4.99024000 & -2.25932600 & 1.72863800 \\
\hline $\mathrm{H}$ & -4.76672300 & -3.29495900 & 0.33801800 \\
\hline $\mathrm{C}$ & -6.39319300 & -1.87490600 & 0.11020700 \\
\hline $\mathrm{H}$ & -6.43157900 & -1.91000300 & -0.98456800 \\
\hline $\mathrm{H}$ & -7.16025100 & -2.55553700 & 0.49938500 \\
\hline $\mathrm{H}$ & -6.66056200 & -0.85553000 & 0.41395700 \\
\hline $\mathrm{P}$ & 1.48257600 & 0.55905800 & -0.59901000 \\
\hline $\mathrm{C}$ & 1.80827900 & -0.95451800 & 0.34131700 \\
\hline $\mathrm{C}$ & 2.62070000 & -1.97890000 & -0.15752800 \\
\hline $\mathrm{C}$ & 1.16857800 & -1.10473800 & 1.57558500 \\
\hline $\mathrm{C}$ & 2.80245900 & -3.13869600 & 0.58559800 \\
\hline $\mathrm{H}$ & 3.10669300 & -1.86766600 & -1.12878800 \\
\hline $\mathrm{C}$ & 1.34719000 & -2.27354400 & 2.31227200 \\
\hline $\mathrm{H}$ & 0.52380900 & -0.31113600 & 1.95955100 \\
\hline $\mathrm{C}$ & 2.16599900 & -3.28567300 & 1.81967700 \\
\hline $\mathrm{H}$ & 3.43778000 & -3.93510700 & 0.19998300 \\
\hline $\mathrm{H}$ & 0.84315900 & -2.39206400 & 3.27039400 \\
\hline $\mathrm{H}$ & 2.30771600 & -4.19936700 & 2.39581100 \\
\hline $\mathrm{C}$ & 3.07803900 & 1.13425200 & -1.25507400 \\
\hline $\mathrm{C}$ & 3.18990400 & 1.68323500 & -2.53452800 \\
\hline $\mathrm{C}$ & 4.19732500 & 1.09965800 & -0.41299200 \\
\hline $\mathrm{C}$ & 4.41423700 & 2.18224000 & -2.97282500 \\
\hline
\end{tabular}

$\begin{array}{cccc}\mathrm{H} & 2.32153000 & 1.71742500 & -3.19376100 \\ \mathrm{C} & 5.41663200 & 1.60038900 & -0.85624500 \\ \mathrm{H} & 4.11838900 & 0.67782500 & 0.59005000 \\ \mathrm{C} & 5.52684200 & 2.14128600 & -2.13635500 \\ \mathrm{H} & 4.49625800 & 2.60378700 & -3.97383000 \\ \mathrm{H} & 6.28431700 & 1.56681500 & -0.19871500 \\ \mathrm{H} & 6.48355000 & 2.53015000 & -2.48304900 \\ \mathrm{Se} & 0.12805700 & 0.07200000 & -2.32758700 \\ \mathrm{H} & 0.74681500 & 1.58256500 & 0.34806900\end{array}$

s-C-IM2

$\begin{array}{llll}\text { Cd } & -1.61532600 & -0.47196800 & -0.67822300\end{array}$

$\begin{array}{llll}\text { O } & 0.07555000 & 2.34652400 & 1.71130400\end{array}$

$\begin{array}{llll}\mathrm{O} & -1.70156800 & 1.58153800 & 0.57717800\end{array}$

$\begin{array}{llll}\text { C } & -1.20144200 & 2.36734200 & 1.37327700\end{array}$

$\begin{array}{llll}\text { C } & -1.96905500 & 3.45737900 & 2.05486500\end{array}$

$\begin{array}{llll}\mathrm{H} & -1.73081400 & 3.40178500 & 3.12781700\end{array}$

$\mathrm{H} \quad-1.53249800 \quad 4.40907100 \quad 1.71141600$

C $\quad-3.46211200 \quad 3.40397200 \quad 1.80124100$

$\begin{array}{llll}\mathrm{H} & -3.96700100 & 4.22703500 & 2.31908800\end{array}$

$\begin{array}{llll}\mathrm{H} & -3.69150300 & 3.48267200 & 0.73221600\end{array}$

$\begin{array}{llll}\mathrm{H} & -3.89237300 & 2.46217900 & 2.16310900\end{array}$

$\begin{array}{llll}\text { O } & -3.84306200 & -0.22315500 & -1.16428200\end{array}$

$\begin{array}{llll}\text { O } & -3.27675800 & -1.26858300 & 0.69663700\end{array}$

$\begin{array}{llll}\text { C } & -4.16271100 & -0.80261700 & -0.08481400\end{array}$

$\begin{array}{llll}\text { C } & -5.60958200 & -0.94868400 & 0.31064700\end{array}$

$\begin{array}{llll}\mathrm{H} & -5.71027700 & -0.46956500 & 1.29672600\end{array}$

$\begin{array}{llll}\mathrm{H} & -5.78163500 & -2.02007800 & 0.49408800\end{array}$

$\begin{array}{llll}\text { C } & -6.61227900 & -0.38761100 & -0.67824000\end{array}$

$\mathrm{H} \quad-6.53325400 \quad-0.88146500 \quad-1.65324400$

$\mathrm{H} \quad-7.63565400 \quad-0.52684300 \quad-0.31115100$

$\begin{array}{llll}\mathrm{H} & -6.45512100 & 0.68438300 & -0.84279300\end{array}$

$\begin{array}{llll}\mathrm{P} & 1.83946500 & 0.09778800 & 0.05054500\end{array}$

$\begin{array}{llll}\text { C } & 2.63223600 & -1.32035100 & 0.92044100\end{array}$

$\begin{array}{llll}\text { C } & 2.27179200 & -2.65696000 & 0.74607800\end{array}$

$\begin{array}{llll}\text { C } & 3.60045700 & -0.99657900 & 1.88267800\end{array}$

$\begin{array}{llll}\text { C } & 2.88069300 & -3.65550600 & 1.50700400\end{array}$

$\mathrm{H} \quad 1.52045600 \quad-2.92245200 \quad 0.00160000$

$\begin{array}{llll}\text { C } & 4.21127300 & -1.99335500 & 2.63543700\end{array}$

$\begin{array}{llll}\mathrm{H} & 3.88797400 & 0.04606000 & 2.03839000\end{array}$

$\begin{array}{llll}\text { C } & 3.85091000 & -3.32836800 & 2.44920700\end{array}$

$\mathrm{H} \quad 2.59408700 \quad-4.69601200 \quad 1.35614600$

$\mathrm{H} \quad 4.96906800 \quad-1.72713200 \quad 3.37172400$

$\begin{array}{llll}\mathrm{H} & 4.32731400 & -4.11057600 & 3.03907600\end{array}$

$\begin{array}{llll}\text { C } & 3.27203800 & 0.81942600 & -0.84405700\end{array}$

$\begin{array}{llll}\text { C } & 3.34087400 & 2.21218200 & -0.94701400\end{array}$ 


$\begin{array}{cccc}\mathrm{C} & 4.26526500 & 0.03682900 & -1.44521900 \\ \mathrm{C} & 4.39079700 & 2.81779000 & -1.63551200 \\ \mathrm{H} & 2.56804200 & 2.82875400 & -0.48320600 \\ \mathrm{C} & 5.31246400 & 0.64186100 & -2.13179400 \\ \mathrm{H} & 4.21686500 & -1.05149600 & -1.37403400 \\ \mathrm{C} & 5.37643700 & 2.03278700 & -2.22751700 \\ \mathrm{H} & 4.43856600 & 3.90384300 & -1.70695000 \\ \mathrm{H} & 6.08200900 & 0.02662300 & -2.59716500 \\ \mathrm{H} & 6.19919200 & 2.50418900 & -2.76417000 \\ \mathrm{Se} & 0.68359900 & -0.83579900 & -1.71605800 \\ \mathrm{H} & 0.55647200 & 1.62368600 & 1.22630800\end{array}$

s-C-i

$\begin{array}{lrrr}\mathrm{Cd} & -1.16561600 & 1.47767000 & -1.79960700 \\ \mathrm{O} & -0.18062800 & 2.51678200 & -0.04712800 \\ \mathrm{O} & 0.64184000 & 2.85990500 & -2.06426700 \\ \mathrm{C} & 0.66672200 & 3.07236500 & -0.81639000 \\ \mathrm{C} & 1.69065800 & 3.99075900 & -0.20725200 \\ \mathrm{H} & 2.18151100 & 3.42595100 & 0.59962300 \\ \mathrm{H} & 1.12803200 & 4.78720900 & 0.30407400 \\ \mathrm{C} & 2.70235300 & 4.56541300 & -1.17877300 \\ \mathrm{H} & 2.21508100 & 5.15277800 & -1.96484000 \\ \mathrm{H} & 3.40791400 & 5.22058100 & -0.65555100 \\ \mathrm{H} & 3.27896700 & 3.77445100 & -1.67141100 \\ \mathrm{P} & -3.80759300 & -0.67365500 & -0.78032800 \\ \mathrm{C} & -4.54697500 & -2.26830700 & -1.33395300 \\ \mathrm{C} & -3.82869100 & -3.43262200 & -1.03666700 \\ \mathrm{C} & -5.74885700 & -2.37094900 & -2.04388300 \\ \mathrm{C} & -4.29989500 & -4.67849200 & -1.44336200 \\ \mathrm{H} & -2.89067300 & -3.35909500 & -0.48245300 \\ \mathrm{C} & -6.22262600 & -3.61700600 & -2.44437300 \\ \mathrm{H} & -6.31811300 & -1.47180900 & -2.28544800 \\ \mathrm{C} & -5.49953300 & -4.77153400 & -2.14553500 \\ \mathrm{H} & -3.73214100 & -5.57773400 & -1.20656400 \\ \mathrm{H} & -7.16015300 & -3.68761300 & -2.99536400 \\ \mathrm{H} & -5.87275800 & -5.74504900 & -2.46191100 \\ \mathrm{C} & -5.32222700 & 0.37443300 & -0.63746700 \\ \mathrm{C} & -5.68458700 & -6.1 .41388300 & -1.49574600 \\ \mathrm{H} & -6.82698100 & 2.17412000 & -1.24189600 \\ \mathrm{H} & -7.26378100 & 0.86801900 & 0.73509800 \\ \mathrm{H} & -1.84222000 & -0.68422300 & 1.17704300 \\ \mathrm{H} & -0.13046000 \\ \mathrm{H} & -1.90264800 & -1.92211300 \\ \mathrm{H} & -1.61011500\end{array}$

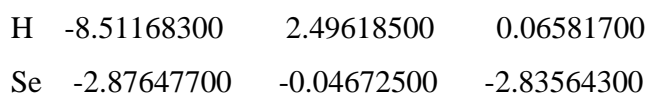

$\begin{array}{crrr}\mathrm{C} & 0.63286900 & -0.13411400 & 0.14700800 \\ \mathrm{O} & 1.58148600 & -0.87998800 & 0.28086700 \\ \mathrm{O} & 0.77075800 & 1.12112000 & -0.30268500 \\ \mathrm{H} & 1.73009100 & 1.27079200 & -0.47278100 \\ \mathrm{C} & -0.78330600 & -0.48852900 & 0.49193100\end{array}$




$\begin{array}{rrrr}\mathrm{C} & -1.85759500 & 0.20400400 & -0.32868800 \\ \mathrm{H} & -0.85858500 & -1.58134500 & 0.42445100 \\ \mathrm{H} & -0.89989400 & -0.24075100 & 1.55993200 \\ \mathrm{H} & -1.74489100 & -0.00697800 & -1.39932900 \\ \mathrm{H} & -2.85023000 & -0.14697400 & -0.02376400 \\ \mathrm{H} & -1.83250000 & 1.29124200 & -0.19772400\end{array}$

